\title{
Late Stage Phosphotyrosine Mimetic Functionalization of Peptides Employing Metallaphotoredox Catalysis
}

Hao Chen, Runyu Mao, Martin Brzozowski, Nghi H. Nguyen* and Brad E. Sleebs*

The Walter and Eliza Hall Institute of Medical Research, Parkville, Victoria, 3052, Australia.

Department of Medical Biology, The University of Melbourne, Parkville, 3010 Victoria, Australia.

Index

$\underline{\text { Page }}$

S2 Figure S1. Proposed catalytic mechanism of the metallaphotoredox conditions

S3 Figure S2. Determination of epimerization under metallaphotoredox conditions

S4 Figure S3 Chiral LC traces of $p$ CMF products $7 \mathbf{a}$ and $7 \mathbf{b}$

S5 $\quad$ Figure S4. Chiral LC traces of $p C M F$ peptides $9 \mathbf{a}, \mathbf{9 b}$ and $\mathbf{9 c}$

S6 Figure S5. Dose response data on compounds against SOCS proteins

S7 - S8 General chemistry methods

S8 - S40 Chemistry procedures

S41 - S42 Biology procedures

S43 References

S44 - S105 NMR or LC spectra of synthesized compounds 
Figure S1. Proposed catalytic mechanism of the metallaphotoredox conditions. The cataylic cycle reflects that proposed by Guan et al. ${ }^{1}$

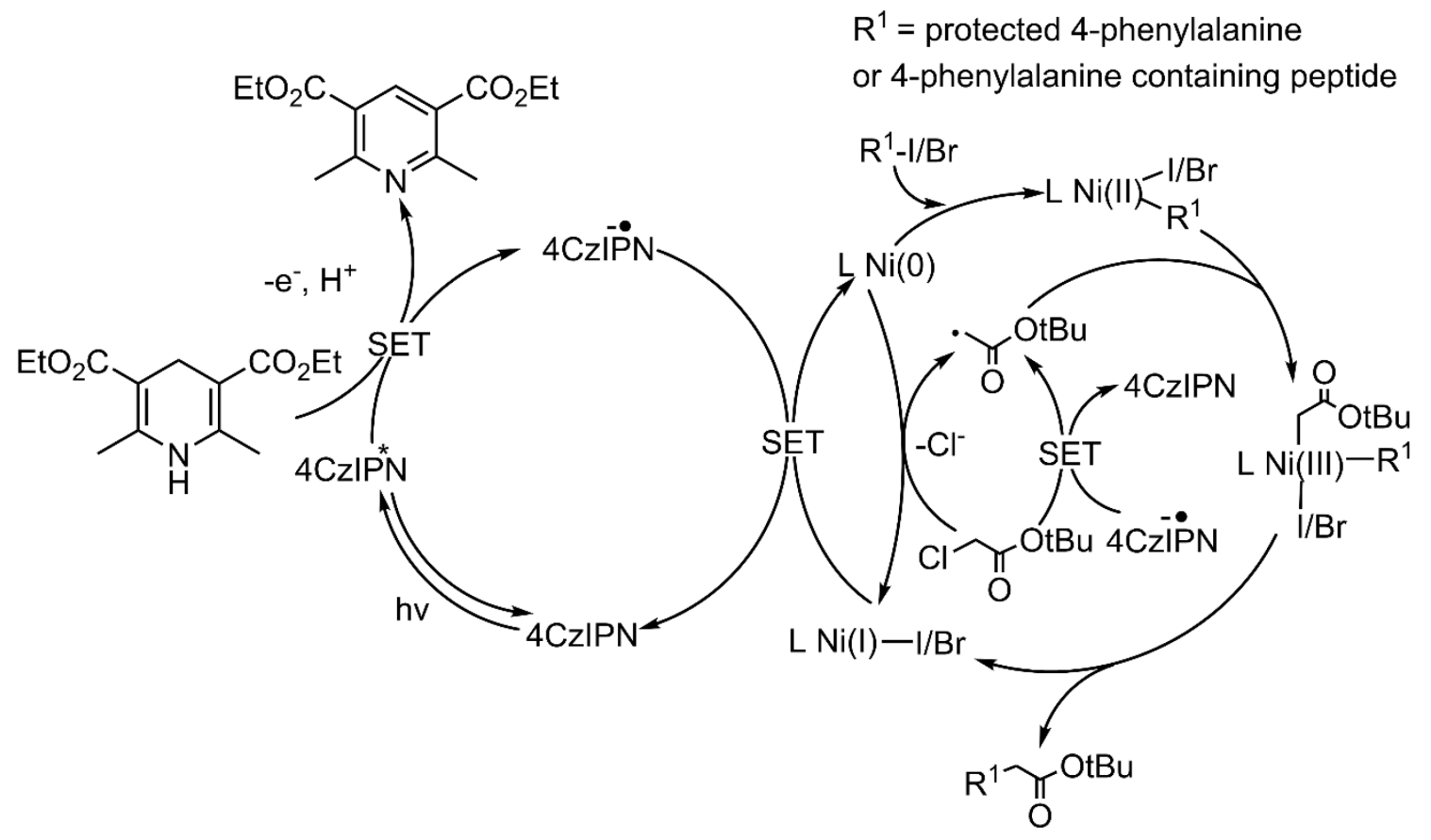


Figure S2. Study to determine epimerization of the amino acid $\alpha$-centre under metallaphotoredox conditions. A. Metallaphotoredox conditions (Table 1, entry 6) were used in independent reactions using the L-isomer $(\boldsymbol{S} 1)$, D-isomer $(\boldsymbol{R} \mathbf{1})$ and a racemic mixture $(1: 1$ of $\boldsymbol{S} / \boldsymbol{R} \mathbf{1})$ of TFA-Phe(4-iodo)-OMe to afford the corresponding TFA- $p \mathrm{CMF}(\mathrm{O} t \mathrm{Bu})-\mathrm{OMe}$ products $(S \mathbf{3}, \boldsymbol{R 3}$ and $\boldsymbol{S} / \boldsymbol{R 3})$. B. Independent chiral-HPLC analysis of the L-isomer $\boldsymbol{S 3}$ (top row) and D-isomer $\boldsymbol{R 3}$ (middle row) and racemic mixture $\boldsymbol{S} / \boldsymbol{R} \mathbf{3}$ (bottom row) products, demonstrated there was no epimerization of the chiral centre under the metallaphotoredox reaction conditions.

A.<smiles>COC(=O)C(Cc1ccc(I)cc1)NC(F)(F)F</smiles>

S1<smiles>CCCCOC(=O)CCl</smiles>

R1

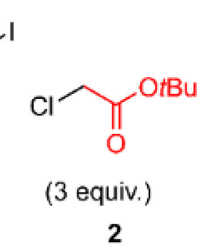

$\mathrm{NiBr}_{2} \cdot \mathrm{DME}(10 \mathrm{~mol} \%)$ 4,4'-dtbbpy (15 $\mathrm{mol} \%)$

$4 \mathrm{CzIPN}(2.5 \mathrm{~mol} \%)$

DIPEA ( 3 equiv.) TFA

HE (3 equiv.)

DMF $(0.1 \mathrm{M})$

blue light, $16 \mathrm{~h}$, r.t<smiles>CCCCOC(=O)Cc1ccc(C[C@H](NC)C(=O)OC)cc1</smiles>

$\mathrm{NiBr}_{2} \cdot \mathrm{DME}(10 \mathrm{~mol} \%)$

4,4'-dtbbpy (15 mol \%)

$4 \mathrm{CzIPN}(2.5 \mathrm{~mol} \%)$

DIPEA (3 equiv.) TFA

HE (3 equiv.)

DMF $(0.1 \mathrm{M})$

blue light, $16 \mathrm{~h}$, r.t<smiles>CCCCOC(=O)Cc1ccc(C[C@H](N)C(=O)OC)cc1</smiles>

$\mathrm{NiBr}_{2} \bullet \mathrm{DME}(10 \mathrm{~mol} \%)$

4,4'-dtbbpy (15 mol \%)<smiles>COC(=O)C(Cc1ccc(I)cc1)NC(C)C</smiles>

4 CZIPN $(2.5 \mathrm{~mol} \%)$

$\stackrel{\mathrm{DIPFA}(3 \text { ( }}{\longrightarrow}$

DIPEA ( 3 equiv.) TFA.
HE ( 3 equiv.)

DMF $(0.1 \mathrm{M})$

blue light, 16 h, r.t

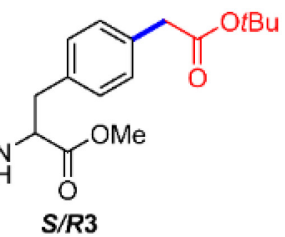

B.
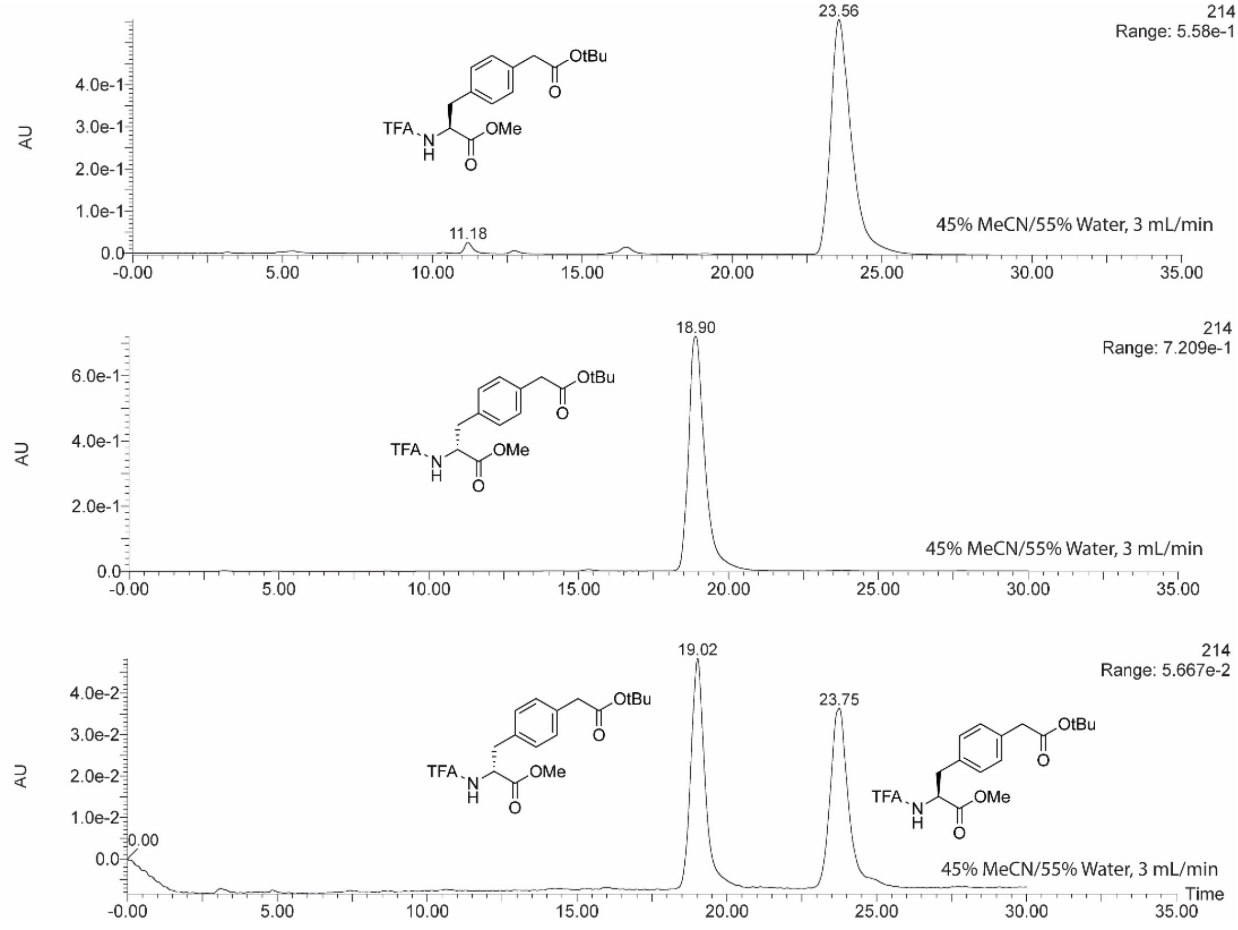
Figure S3. Chiral LC traces showing no stereo selectivity in formation of the new aryl $\alpha$ carbon in $p$ CMF products $\mathbf{7 a}$ and $\mathbf{7 b}$ under metallaphotoredox conditions. Middle and bottom panels show an approximate 50:50 mixture of epimers at the aryl $\alpha$-carbon of compounds 7a (peaks 8.03 and 14.05) and $7 \mathbf{b}$ (peaks 8.58 and 9.89). Top panel is a blank showing the peak at 4.94 is a column artefact.

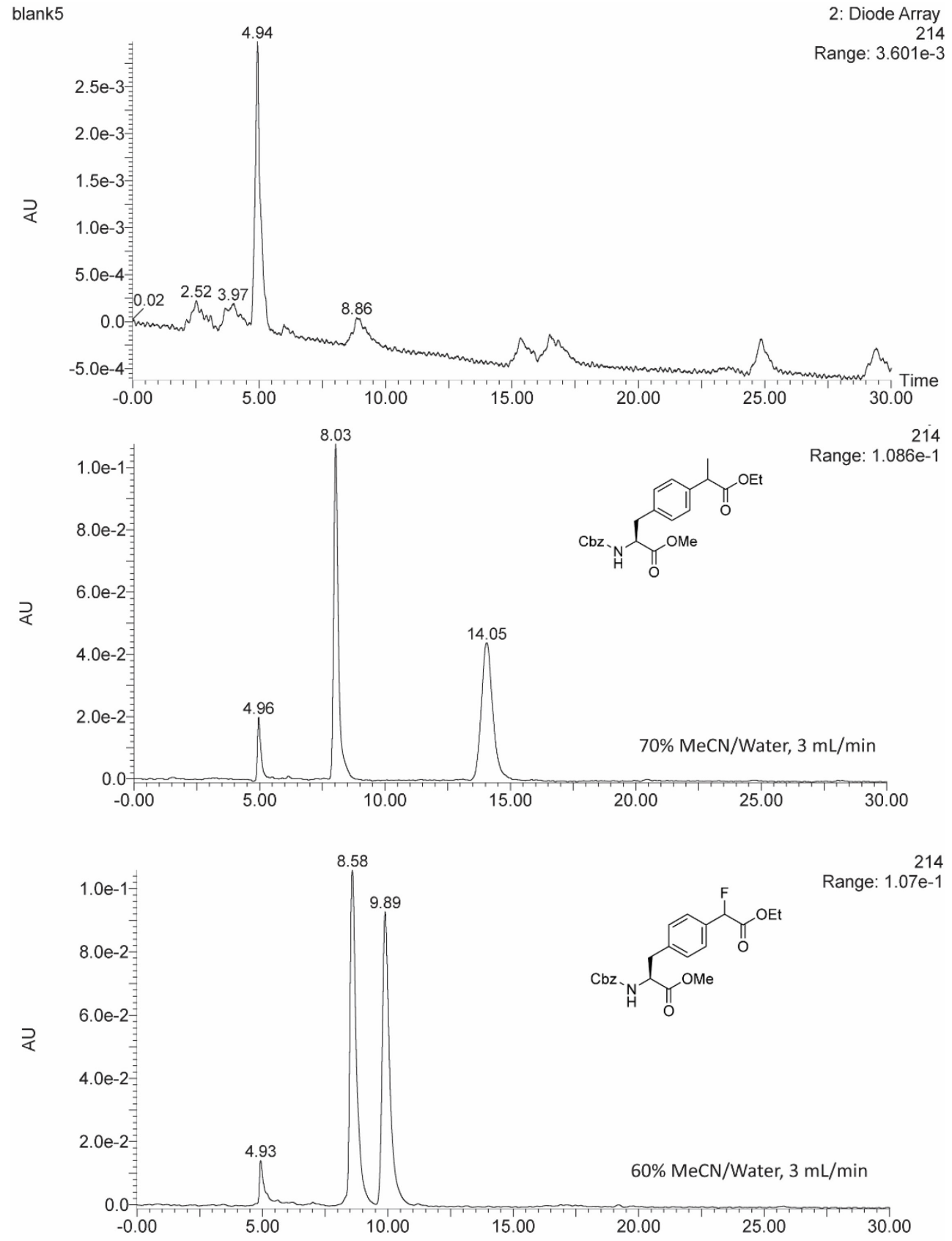


Figure S4. Chiral LC traces showing no stereo epimerization in the formation of the representative $p C M F$ containing peptides 9a (top panel), 9b (middle panel) and 9c (bottom panel) under metallaphotoredox conditions. All LC traces show a single peak supporting the evidence in Figure S2 that no epimerization is observed under metallaphotoredox conditions. Peak at 4.92 is a column artefact (see blank LC trace in top panel of Figure S2).
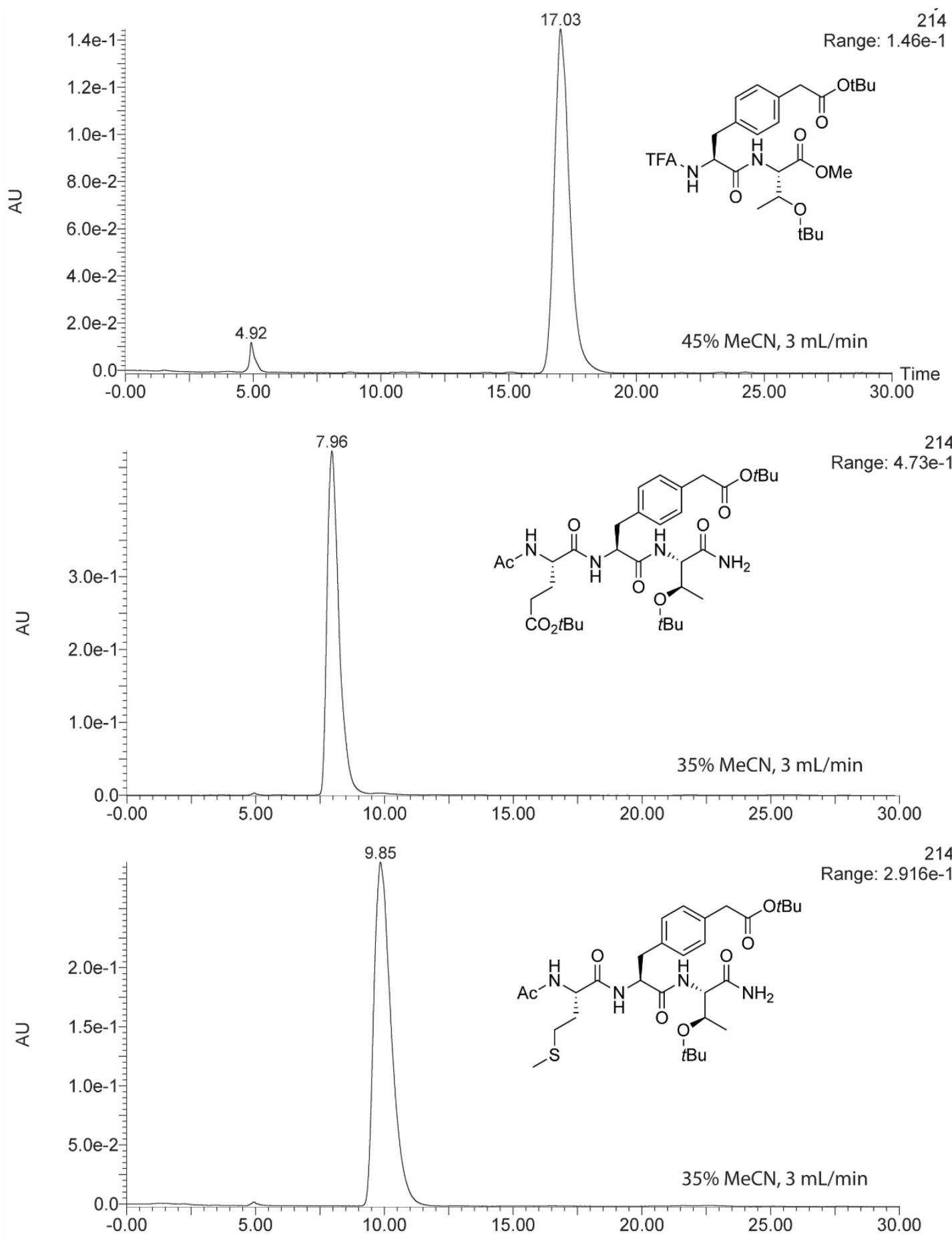
Figure S5. The competition SPR analysis of compounds 12a, 12b and 12c against SOCS-SH2 domains proteins. A. The chemical structures of compounds 12a, 12b and 12c. B. Dose response curves.

A.<smiles>CC(=O)N[C@@H](CCC(=O)O)C(=O)N[C@@H](Cc1ccc(OP(=O)(O)O)cc1)C(=O)N[C@@H](CO)C(=O)N[C@@H](C(=O)N[C@H](C(N)=O)C(C)C)C(C)C</smiles>

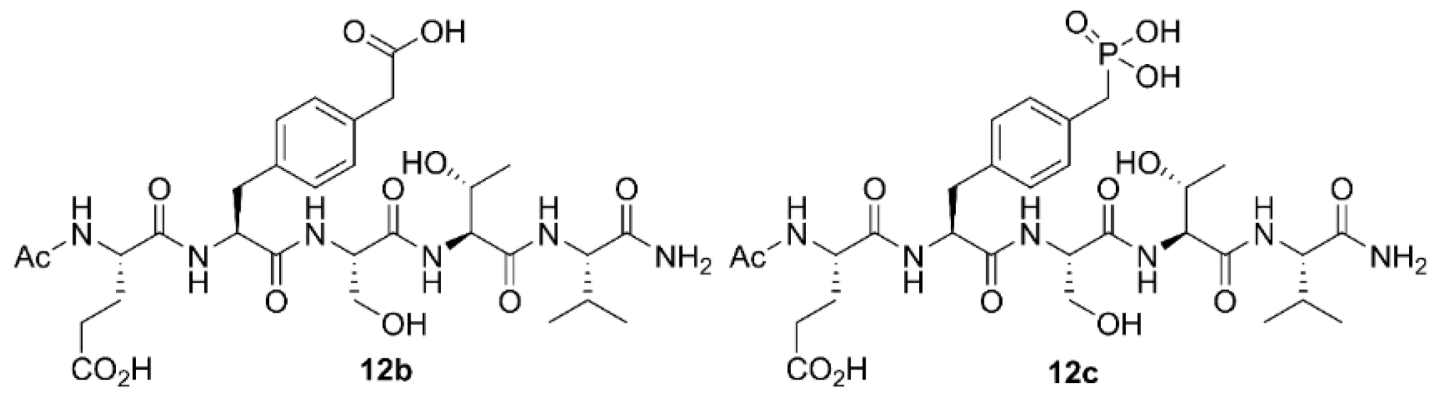

B.
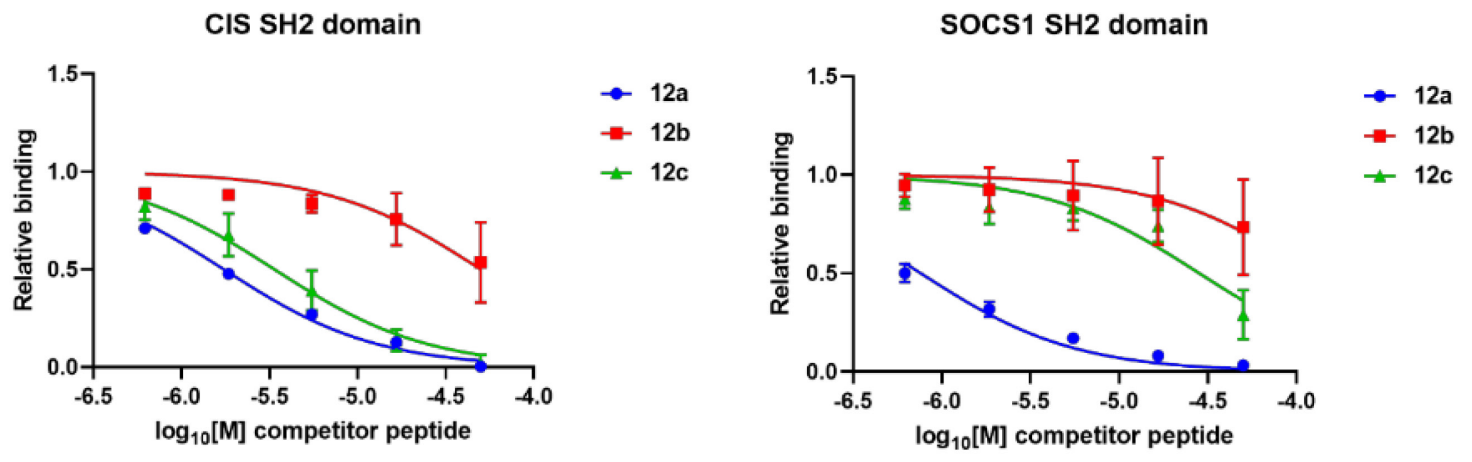

SOcS2 SH2 domain

SOCS2 SH2 domain
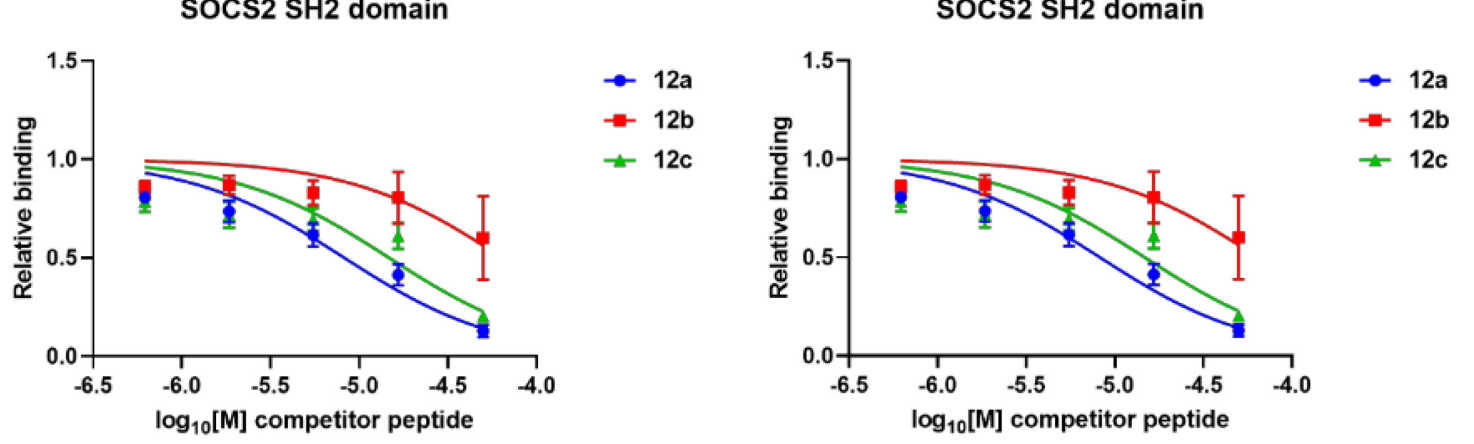


\section{General Chemistry Methods.}

Solvents were obtained commercially and used without further purification. Chromatography was performed using SiliCycle SiliaSep silica gel flash cartridges $60 \AA$ (particle size 40-63 $\mu \mathrm{m}$ ) in combination with an automated purification system unless otherwise stated. NMR spectra were recorded on a Bruker Avance DRX 300. Chemical shifts are reported in parts per million (ppm) and referenced to the appropriate solvent peak. NMR solvents MeOD- $d_{4}$, DMSO- $d_{6}$, and $\mathrm{CDCl}_{3}$ contain residual $\mathrm{H}_{2} \mathrm{O}$.

LCMS analysis were performed on an Agilent LCMS system equipped with an Agilent G6120B Mass Detector, 1260 Infinity G1312B Binary pump, 1260 Infinity G1367E HiPALS autosampler, and 1260 Infinity G4212B Diode Array Detector. The LCMS conditions were as follows: column: Luna Omega $(1.6 \mu \mathrm{m}, \mathrm{C} 18,50 \times 2.1 \mathrm{~mm})$; injection volume: $1 \mu \mathrm{L}$; gradient: 5-100\% B over 3.8 min (solvent A: water/0.1\% formic acid; solvent B: ACN/ 0.1\% formic acid); acquisition time: $4.1 \mathrm{~min}$; flow rate: $1 \mathrm{~mL} / \mathrm{min}$; detection: 254 and $214 \mathrm{~nm}$. Unless otherwise noted, all compounds were found to be $>95 \%$ pure by this method.

Preparative LCMS purification was performed using a Waters preparative HPLC system equipped with Waters ZQ 3100 -Mass Detector, Waters 2545-Pump, Waters SFO System Fluidics Organizer, Waters 2996 Diode Array Detector and Waters 2767 Sample Manager. The preparative-HPLC conditions were as follows: XBridge BEH C18 OBD Prep Column (130Å, $5 \mu \mathrm{m}, 19 \mathrm{~mm}$ X $100 \mathrm{~mm}$ ); injection volume: $1 \mathrm{~mL}$; gradient is variable over 20 min depending on each compound (solvent A: water/0.1\% formic acid; solvent $\mathrm{B}$ : $\mathrm{ACN} / 0.1 \%$ formic acid); flow rate, $20 \mathrm{~mL} / \mathrm{min}$; detection 100 to $600 \mathrm{~nm}$.

Chiral HPLC analysis was performed with the aforementioned Waters system using a Phenomenex Lux 5u Cellulose-3 (250 x $10 \mathrm{~mm})$ column and $214 \mathrm{~nm}$ detection. The flow rate and gradient were followed as indicated in individual LC traces shown on Figures S2-S4.

High resolution mass spectra were acquired at the The Bio21 Mass Spectrometry and Proteomics Facility using a Thermo Scientific ${ }^{\mathrm{TM}}$ nano-LC OrbiTrap Exactive ${ }^{\mathrm{TM}}$ Plus Mass Spectrometer with electrospray ionisation (ESI).

Photoredox reactions were performed using a HepatoChem PhotoRedOx Box, (HCK1006-01016), equipped with HepatoChem EvoluChem ${ }^{\mathrm{TM}}$ LED spotlights (18W, 450 nM, HCK101201-002). The distance between the light source and the reaction vessel was 7 to $10 \mathrm{~cm}$. For more details of the photoreactor and light source intensity and wavelength range, please refer to supplier website: https://www.hepatochem.com/. 
Solid phase peptide synthesis was performed using a CEM Liberty Blue automatic synthesizer equipped with CEM Discover microwave reactor and CEM $30 \mathrm{~mL}$ 2-piece reaction vessel (P/N 551605). For the coupling step, the reaction vessel was initially heated by microwave irradiation to $75^{\circ} \mathrm{C}(175 \mathrm{~W})$ within $15 \mathrm{sec}$ and further increased to $90{ }^{\circ} \mathrm{C}(30 \mathrm{~W})$ for $230 \mathrm{sec}$. For the deprotection step, the reaction vessel was heated by microwave irradiation to $75{ }^{\circ} \mathrm{C}$ (70W) for $180 \mathrm{sec}$.

\section{General Chemistry Procedures}

\section{2,4,5,6-Tetra(9H-carbazol-9-yl)isophthalonitrile (4CzIPN)}

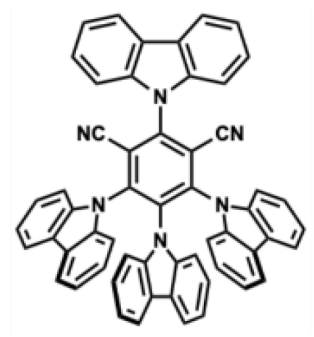

The title compound was prepared via a modified literature procedure. ${ }^{2}$ A $250 \mathrm{~mL}$ RBF equipped with a reflux condenser, $\mathrm{N}_{2}$ inlet and a stir bar was charged with $9 H$-carbazole (1.67 $\mathrm{g}, 10.0 \mathrm{mmol})$ and anhydrous THF $(40 \mathrm{~mL})$. The homogeneous solution was cooled to $0{ }^{\circ} \mathrm{C}$ (ice bath) and then $\mathrm{NaH}$ (60\% dispersion in mineral oil, $600 \mathrm{mg}, 12.0 \mathrm{mmol}$ ) was added portion wise over $10 \mathrm{~min}$. After $30 \mathrm{~min}$, tetrafluoroisophthalonitrile (400 mg, $2.0 \mathrm{mmol}$ ) was added and the mixture stirred at $65^{\circ} \mathrm{C}$ for a further $24 \mathrm{~h}$. The reaction mixture was concentrated under reduced pressure and the residue was triturated with $\mathrm{Et}_{2} \mathrm{O}$ and the solid collected. The resulting solid was suspended in $\mathrm{CHCl}_{3}(150 \mathrm{~mL})$, filtered and the filtrate concentrated. The crude product was washed with 9:1 heptane/acetone $(3 \times 10 \mathrm{~mL})$, pentane $(2 \times 10 \mathrm{~mL})$ and then dried at $50{ }^{\circ} \mathrm{C}$ under vacuum to afford the title compound as a bright yellow solid $(1.38 \mathrm{~g}, 87 \%) .{ }^{1} \mathrm{H}$ NMR (300 MHz, CDCl 3$): \delta 8.23(\mathrm{~d}, \mathrm{~J} 7.8 \mathrm{~Hz}, 2 \mathrm{H}), 7.77$ - $7.63(\mathrm{~m}, 8 \mathrm{H}), 7.49$ (ddd, J 8.1, 6.4, $1.9 \mathrm{~Hz}, 2 \mathrm{H}), 7.38-7.29(\mathrm{~m}, 2 \mathrm{H}), 7.27-7.15(\mathrm{~m}, 8 \mathrm{H}), 7.15-7.01(\mathrm{~m}, 8 \mathrm{H}), 6.82$ (ddd, J 7.4, $6.8,1.0 \mathrm{~Hz}, 4 \mathrm{H}), 6.63$ (ddd, J 8.2, 7.2, $1.2 \mathrm{~Hz}, 2 \mathrm{H}$ ). The spectrum matches the previously reported data. $^{2}$ 
General procedure A. Synthesis of $N$-protected amino esters.

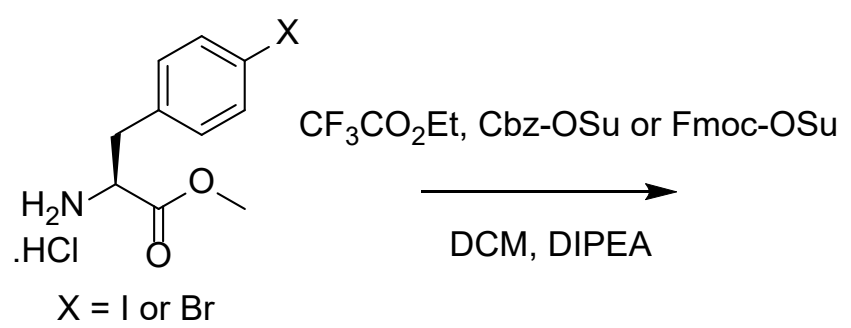<smiles>[R]NC(Cc1ccc([X])cc1)C(=O)OC</smiles>

To a solution of 4-halo phenylalanine derivate $(10 \mathrm{mmol})$ in DCM $(30 \mathrm{~mL})$ was added DIPEA $(11 \mathrm{mmol})$ and $\mathrm{CF}_{3} \mathrm{CO}_{2} \mathrm{Et}(20 \mathrm{mmol}), \mathrm{Cbz}-\mathrm{OSu}$ or Fmoc-OSu $(11 \mathrm{mmol})$. The reaction mixture was stirred at room temperature for $18 \mathrm{~h}$. The reaction mixture was concentrated to dryness and then purified by flash column chromatography gradient eluting with $0-25 \% \mathrm{EtOAc} / \mathrm{n}$ heptane.

\section{General procedure B. Synthesis of pTyr analogues.}<smiles>[R]Cc1ccc(CC(N[R])C(=O)O[R])cc1</smiles>

To a $4 \mathrm{~mL}$ vial equipped with stir bar was added 4-halide phenylalanine derivative $(0.1 \mathrm{mmol})$, Hantzsch ester (76 mg, $0.3 \mathrm{mmol}$ ), 4CzIPN (2 mg, $0.0005 \mathrm{mmol}, 2.5 \mathrm{~mol} \%$ ) and anhydrous $\operatorname{DMF}(0.5 \mathrm{~mL})$. To a separate $4 \mathrm{~mL}$ vial was added $\mathrm{NiBr}_{2} \bullet$ glyme $(3.1 \mathrm{mg}, 0.01 \mathrm{mmol}, 10 \mathrm{~mol} \%)$, 4,4'-dtbbpy (4.02 mg, $0.015 \mathrm{mmol}, 15 \mathrm{~mol} \%$ ) and anhydrous DMF (0.5 mL). The vial with the pre-catalyst was sealed and sonicated for $15 \mathrm{~min}$. The pre-catalyst solution was then transferred into the reaction vial using a syringe. The reaction vial was then sealed and purged with nitrogen. The alkyl chloride $(0.3 \mathrm{mmol})$ and DIPEA $(0.052 \mathrm{~mL}, 0.3 \mathrm{mmol})$ were then added. The final reaction mixture was sealed with parafilm, stirred, and irradiated under blue light for $16 \mathrm{~h}$. The reaction mixture was purified by flash chromatography to afford the product. 


\section{General procedure C. Preparation of peptides by solid phase synthesis.}

Peptides were synthesized by solid phase using standard Fmoc chemistry on Rink Amide resin (side chain deprotected) or Sieber resin (side chain protected) using CEM Liberty Blue automated microwave peptide synthesizer. Standard amino acids $(1.25 \mathrm{mmol})$ were coupled to Rink Amide AM resin $(0.316 \mathrm{~g}, 0.79 \mathrm{mmol} / \mathrm{g}, 0.25 \mathrm{mmol})$ or Sieber resin $(0.423 \mathrm{~g}, 0.59$ $\mathrm{mmol} / \mathrm{g}, 0.25 \mathrm{mmol})$ iteratively using DIC (1 mmol), Oxyma $(1 \mathrm{mmol})$ and DIPEA $(0.4 \mathrm{mmol})$ in DMF $(9 \mathrm{~mL})$ for $4 \mathrm{~min}$ at $90{ }^{\circ} \mathrm{C}$, except for Fmoc-Arg(Pbf)-OH which was double coupled at $90{ }^{\circ} \mathrm{C}$ for 4 min. The Fmoc protecting group was removed after each coupling cycle using a mixture containing 4-methyl-piperidine $(20 \% \mathrm{v} / \mathrm{v})$ and Oxyma $(0.1 \mathrm{mmol})$ in DMF $(2 \times 5 \mathrm{~mL})$ with microwave irradiation for $2 \mathrm{~min}$ at $75^{\circ} \mathrm{C}$. The resin was then washed with DMF $(3 \times 5$ $\mathrm{mL}$ ). Oxyma was added to the deprotection mixture to minimize aspartimide formation.

The N-terminus of peptides was acylated with a mixture of acetic anhydride $(10 \% \mathrm{v} / \mathrm{v})$ and DIPEA $(10 \% \mathrm{v} / \mathrm{v})$ in DMF $(10 \mathrm{~mL})$ for $30 \mathrm{~min}$.

For Rink amide resin, peptides were cleaved from the resin and globally deprotected using a cocktail containing TFA, DMB, TIPS (95:2.5:2.5, vol/vol/vol) for $1 \mathrm{~h}$ at $40^{\circ} \mathrm{C}$ using the CEM Razor unit. The resin was filtered, and the filtrate was triturated three times with cold $\mathrm{Et}_{2} \mathrm{O}$ (40 $\mathrm{mL}$ ). The supernatant was decanted, and the solid pellet was then dried at room temperature for $20 \mathrm{~h}$. The peptides were then purified by preparative HPLC.

For Sieber resin, peptides were cleaved from the resin for $3 \times 30$ min using $1 \%$ TFA in DCM. The resin was filtered and filtrates were combined and evaporated. Peptides were then purified by flash chromatography.

\section{General procedure D. Synthesis of pTyr mimetic containing peptides.}

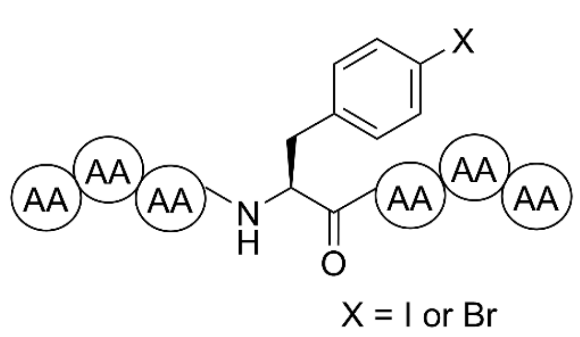

$\mathrm{X}=\mathrm{I}$ or $\mathrm{Br}$

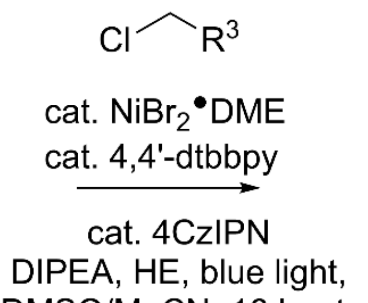

DMSO/MeCN, 16 h, r.t.

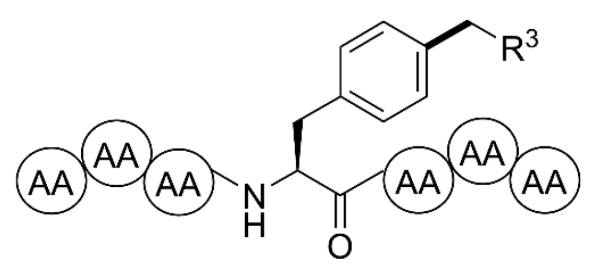


To a $4 \mathrm{~mL}$ vial equipped with stir bar was added 4-halo phenylalanine containing peptides $(0.1$ mmol), Hantzsch ester (76 mg, $0.3 \mathrm{mmol}$ ), 4CzIPN (2 mg, $0.0005 \mathrm{mmol}, 2.5 \% \mathrm{~mol}$ ) and dry DMSO $(0.5 \mathrm{~mL})$. To a separate $4 \mathrm{~mL}$ vial was added $\mathrm{NiBr}_{2} \bullet \mathrm{glyme}(3.1 \mathrm{mg}, 0.01 \mathrm{mmol}, 10 \%$ mol), 4,4'-dtbbpy (4.02 mg, $0.015 \mathrm{mmol}, 15 \% \mathrm{~mol})$ and anhydrous MeCN (0.5 mL). The catalyst vial was sealed and sonicated for $15 \mathrm{~min}$. The precatalytst solution was then transferred to the reaction vial by syringe. The reaction vial was then sealed and purged with nitrogen. The alkyl chloride $(0.3 \mathrm{mmol})$ and DIPEA $(0.052 \mathrm{~mL}, 0.3 \mathrm{mmol})$ were then added. The final reaction mixture was sealed with parafilm, stirred and irradiated under blue light for $16 \mathrm{~h}$. The reaction mixture was purified by flash chromatography or preparative HPLC to afford the product.

(S)-Methyl 3-(4-iodophenyl)-2-(2,2,2-trifluoroacetamido)propanoate (1)<smiles>COC(=O)[C@H](Cc1ccc(I)cc1)NC(C)C</smiles>

General procedure A was followed using L-4-iodo-phenylalanine methyl ester (3 g, $10 \mathrm{mmol})$. The crude product was purified by flash column chromatography gradient eluting with $0-25 \%$ EtOAc/n-heptane to afford 1 as a white solid (3.5 g, 86\%). ${ }^{1} \mathrm{H}$ NMR (300 MHz, $\left.\mathrm{CDCl}_{3}\right) \delta 7.64$ (d, J 8.3 Hz, 2H), 6.81 (d, J 8.3 Hz, 2H), $4.91-4.81(\mathrm{~m}, 1 \mathrm{H}), 3.79(\mathrm{~s}, 3 \mathrm{H}), 3.26-3.06(\mathrm{~m}, 2 \mathrm{H})$. The spectrum matches previous reported data. ${ }^{3}$

\section{(S)-Methyl 2-(((benzyloxy)carbonyl)amino)-3-(4-iodophenyl)propanoate (4a)}<smiles>COC(=O)[C@H](Cc1ccc(I)cc1)NC(=O)OCc1ccccc1</smiles>

General procedure A was followed using L-4-iodo-phenylalanine methyl ester (3 g, $10 \mathrm{mmol})$. The crude product was purified by flash column chromatography gradient eluting with $0-25 \%$ 
EtOAc/n-heptane to afford $4 \mathbf{a}$ as a white solid $(3.3 \mathrm{~g}, 73 \%) .{ }^{1} \mathrm{H}$ NMR $\left(300 \mathrm{MHz}, \mathrm{CDCl}_{3}\right) \delta$ $7.58(\mathrm{~d}, J 8.3 \mathrm{~Hz}, 2 \mathrm{H}), 7.35$ (dq, $J 7.3,5.3,3.7 \mathrm{~Hz}, 5 \mathrm{H}), 6.83$ (d, $J 8.3 \mathrm{~Hz}, 2 \mathrm{H}), 5.21$ (d, $J 8.2$ $\mathrm{Hz}, 1 \mathrm{H}), 5.16-5.00(\mathrm{~m}, 2 \mathrm{H}), 4.64(\mathrm{q}, J 6.5 \mathrm{~Hz}, 1 \mathrm{H}), 3.05(\mathrm{qd}, J 13.9,5.9 \mathrm{~Hz}, 2 \mathrm{H})$. The spectrum matches previous reported data. ${ }^{4}$

(S)-Methyl 2-((((9H-fluoren-9-yl)methoxy)carbonyl)amino)-3-(4-iodophenyl)propanoate (4c)<smiles>COC(=O)C(Cc1ccc(I)cc1)NC(F)F</smiles>

General procedure A was followed using L-4-iodo-phenylalanine methyl ester (3.0 g, $10 \mathrm{mmol})$. The crude product was purified by flash column chromatography gradient eluting with $0-25 \%$ EtOAc/n-heptane to afford $\mathbf{4 c}$ as a white solid (3.4 g, $81 \%) .{ }^{1} \mathrm{H} \mathrm{NMR}\left(300 \mathrm{MHz}, \mathrm{CDCl}_{3}\right) \delta$ $7.78(\mathrm{~d}, J 7.5 \mathrm{~Hz}, 2 \mathrm{H}), 7.64-7.52(\mathrm{~m}, 4 \mathrm{H}), 7.41(\mathrm{dd}, J 7.9,6.6 \mathrm{~Hz}, 2 \mathrm{H}), 7.32(\mathrm{tt}, J 7.4,1.1 \mathrm{~Hz}$, 2H), 6.82 (d, J 7.9 Hz, 2H), 5.23 (d, J $8.1 \mathrm{~Hz}, 1 \mathrm{H}), 4.64$ (q, J $6.2 \mathrm{~Hz}, 1 \mathrm{H}), 4.42$ (ddd, J 31.0, 10.6, $6.8 \mathrm{~Hz}, 2 \mathrm{H}), 4.21(\mathrm{t}, J 6.9 \mathrm{~Hz}, 1 \mathrm{H}), 3.73(\mathrm{~s}, 3 \mathrm{H}), 3.04(\mathrm{dt}, J 13.9,6.9 \mathrm{~Hz}, 2 \mathrm{H})$. The spectrum matches previous reported data. ${ }^{5}$

(S)-Methyl 2-((tert-butoxycarbonyl)amino)-3-(4-iodophenyl)propanoate (4d)<smiles>COC(=O)[C@H](Cc1ccc(I)cc1)NC(=O)OCc1ccccc1</smiles>

General procedure A was followed using L-4-iodo-phenylalanine methyl ester ( $3 \mathrm{~g}, 10 \mathrm{mmol})$. The crude product was purified by flash column chromatography gradient eluting with $0-25 \%$ EtOAc/n-heptane to afford $4 \mathbf{d}$ as a white solid (3.6 g, $90 \%) .{ }^{1} \mathrm{H}$ NMR $\left(300 \mathrm{MHz}, \mathrm{CDCl}_{3}\right) \delta$ $7.61(\mathrm{~d}, J 8.3 \mathrm{~Hz}, 2 \mathrm{H}), 6.87$ (d, J $8.3 \mathrm{~Hz}, 2 \mathrm{H}), 4.97$ (d, J 8.3 Hz, 1H), 4.56 (d, J 7.6 Hz, 1H), 
$3.71(\mathrm{~s}, 3 \mathrm{H}), 3.02(\mathrm{qd}, J 13.8,6.0 \mathrm{~Hz}, 2 \mathrm{H}), 1.41(\mathrm{~s}, 9 \mathrm{H})$. The spectrum matches previous reported data. ${ }^{6}$

(S)-Methyl 2-(((benzyloxy)carbonyl)amino)-3-(4-bromophenyl)propanoate (6)<smiles>COC(=O)[C@H](Cc1ccc(Br)cc1)NC(=O)OCc1ccccc1</smiles>

General procedure A was followed using L-4-bromo-phenylalanine methyl ester (2.6 g, 10 mmol). The crude product was purified by flash column chromatography gradient eluting with 0-25\% EtOAc/n-heptane to afford 6 as a white solid (3.1 g, 79\%). ${ }^{1} \mathrm{H}$ NMR (300 $\left.\mathrm{MHz}, \mathrm{CDCl}_{3}\right)$ $\delta 7.36(\mathrm{tdd}, J 8.4,6.3,2.7 \mathrm{~Hz}, 7 \mathrm{H}), 6.96(\mathrm{~d}, J 8.4 \mathrm{~Hz}, 2 \mathrm{H}), 5.28-5.02(\mathrm{~m}, 3 \mathrm{H}), 4.64(\mathrm{q}, J 6.4$ $\mathrm{Hz}, 1 \mathrm{H}), 3.72(\mathrm{~s}, 3 \mathrm{H}), 3.06(\mathrm{qd}, J 13.9,5.9 \mathrm{~Hz}, 2 \mathrm{H}) .{ }^{13} \mathrm{C} \mathrm{NMR}\left(75 \mathrm{MHz}, \mathrm{CDCl}_{3}\right) \delta 171.8,155.7$, 136.3, 134.9, 131.9, 131.1, 128.7, F128.4, 128.3, 121.3, 67.2, 54.7, 52.6, 37.9. HRMS m/z: (ESI) $\left([\mathrm{M}-\mathrm{H}]^{+}\right)$Calcd. for $\mathrm{C}_{18} \mathrm{H}_{19}{ }^{79} \mathrm{BrNO}_{4}:$ 392.0497; Found: 392.0494 .

(S)-Methyl-3-(4-(2-(tert-butoxy)-2-oxoethyl)phenyl)-2-(2,2,2trifluoroacetamido)propanoate (3)<smiles>CCCCOC(=O)Cc1ccc(C[C@H](NC(C)C)C(=O)OC)cc1</smiles>

General procedure B was followed using $1(40.1 \mathrm{mg}, 0.1 \mathrm{mmol})$ and tert-butyl chloroacetate (42.9 $\mu \mathrm{L}, 0.3 \mathrm{mmol})$. The crude product was purified by flash column chromatography gradient eluting with 0-25\% EtOAc/n-heptane to afford 3 as a colourless oil (28.4 mg, $73 \%)$. ${ }^{1} \mathrm{H}$ NMR $\left(300 \mathrm{MHz}^{\mathrm{CDCl}} \mathrm{CD}_{3} \delta 7.21(\mathrm{~d}, J 8.1 \mathrm{~Hz}, 2 \mathrm{H}), 7.02(\mathrm{~d}, J 8.1 \mathrm{~Hz}, 2 \mathrm{H}), 6.84(\mathrm{~d}, J 7.9 \mathrm{~Hz}, 1 \mathrm{H}), 4.92\right.$ - $4.79(\mathrm{~m}, 1 \mathrm{H}), 3.77$ (s, 3H), 3.49 (s, 2H), $3.28-3.06(\mathrm{~m}, 2 \mathrm{H}), 1.42(\mathrm{~s}, 9 \mathrm{H}) .{ }^{13} \mathrm{C}$ NMR (75 $\left.\mathrm{MHz}, \mathrm{CDCl}_{3}\right) \delta 170.9,170.5,156.7\left(\mathrm{t}, J_{\mathrm{C}-\mathrm{F}} 37.7 \mathrm{~Hz}\right), 134.3,133.2,129.8,129.4,115.7\left(\mathrm{~d}, J_{\mathrm{C}-\mathrm{F}}\right.$ 
287.7 Hz), 81.1, 53.6, 52.9, 42.4, 37.0, 28.1. ${ }^{19} \mathrm{~F}$ NMR (282 MHz, $\left.\mathrm{CDCl}_{3}\right) \delta$-75.93. HRMS (ESI) m/z: $\left([\mathrm{M}+\mathrm{H}]^{+}\right)$Calcd. for $\mathrm{C}_{18} \mathrm{H}_{24} \mathrm{~F}_{3} \mathrm{NO}_{5}: 390.1528$; Found: 390.1523 .

(S)-Methyl-2-(((benzyloxy)carbonyl)amino)-3-(4-(2-(tert-butoxy)-2oxoethyl)phenyl)propanoate (5a)<smiles>CCCCOC(=O)Cc1ccc(C[C@H](NC(=O)OCc2ccccc2)C(=O)OC)cc1</smiles>

General procedure B was followed using $4 \mathbf{a}(43.9 \mathrm{mg}, 0.1 \mathrm{mmol})$ and tert-butyl chloroacetate $(42.9 \mu \mathrm{L}, 0.3 \mathrm{mmol})$. The crude product was purified by flash column chromatography gradient eluting with 0-25\% EtOAc/n-heptane to afford 5a as a colourless oil (34.2 mg, 80 \%). ${ }^{1} \mathrm{H}$ NMR $\left(300 \mathrm{MHz}, \mathrm{CDCl}_{3}\right) \delta 7.39-7.27(\mathrm{~m}, 5 \mathrm{H}), 7.21-7.15(\mathrm{~m}, 2 \mathrm{H}), 7.08-7.02(\mathrm{~m}, 2 \mathrm{H}), 5.30(\mathrm{~d}, J$ $8.3 \mathrm{~Hz}, 1 \mathrm{H}), 5.09$ (d, $J 1.6 \mathrm{~Hz}, 2 \mathrm{H}), 4.65$ (dt, $J 8.3,6.0 \mathrm{~Hz}, 1 \mathrm{H}), 3.70$ (s, 3H), 3.49 (s, 2H), 3.10 (q, $J 7.3,6.5 \mathrm{~Hz}, 2 \mathrm{H}), 1.43(\mathrm{~s}, 9 \mathrm{H}) .{ }^{13} \mathrm{C} \mathrm{NMR}\left(75 \mathrm{MHz}, \mathrm{CDCl}_{3}\right) \delta 172.0,170.9,155.7,136.3$, 134.3, 133.6, 129.5, 129.4, 128.5, 128.2, 128.1, 80.9, 67.1, 54.9, 52.3, 42.3, 37.9, 28.1. HRMS (ESI) m/z: $\left([\mathrm{M}+\mathrm{H}]^{+}\right)$Calcd. for $\mathrm{C}_{24} \mathrm{H}_{30} \mathrm{NO}_{6}$ : 428.2073; Found: 428.2070.

(S)-2-(((Benzyloxy)carbonyl)amino)-3-(4-(2-(tert-butoxy)-2-oxoethyl)phenyl)propanoic acid (5b)<smiles>CCCCOC(=O)Cc1ccc(C[C@H](NC(=O)OCc2ccccc2)C(=O)O)cc1</smiles>

General procedure B was followed using $\mathbf{4 b}(42.5 \mathrm{mg}, 0.1 \mathrm{mmol})$ and tert-butyl chloroacetate $(42.9 \mu \mathrm{L}, 0.3 \mathrm{mmol})$. The crude product was purified by flash column chromatography gradient eluting with $0-25 \%$ EtOAc/n-heptane then $0-10 \% \mathrm{MeOH} / \mathrm{DCM}$ and further purified by preparative HPLC to afford $\mathbf{5 b}$ as a colourless oil $(22.7 \mathrm{mg}, 55 \%) .{ }^{1} \mathrm{H}$ NMR $\left(300 \mathrm{MHz}, \mathrm{CDCl}_{3}\right)$ $\delta 7.34(\mathrm{~s}, 5 \mathrm{H}), 7.18(\mathrm{~d}, J 8.1 \mathrm{~Hz}, 2 \mathrm{H}), 7.10(\mathrm{~d}, J 8.1 \mathrm{~Hz}, 2 \mathrm{H}), 5.21(\mathrm{~d}, J 8.2 \mathrm{~Hz}, 1 \mathrm{H}), 5.10$ (s, 
2H), 4.68 (q, J 6.4 Hz, 1H), 3.49 (s, 2H), $3.24-3.04$ (m, 2H), 1.43 (s, 9H). ${ }^{13} \mathrm{C}$ NMR (75 MHz, $\left.\mathrm{CDCl}_{3}\right) \delta 175.2,171.3,156.0,136.2,134.2,133.8,129.7,129.6,128.7,128.4,128.3,81.2,67.3$, 54.7, 42.3, 37.5, 28.2. HRMS (ESI) m/z: $\left([\mathrm{M}+\mathrm{H}]^{+}\right)$Calcd. for $\mathrm{C}_{23} \mathrm{H}_{28} \mathrm{NO}_{6}$ : 414.1916; Found: 414.1898 .

(S)-Methyl-2-((((9H-fluoren-9-yl)methoxy)carbonyl)amino)-3-(4-(2-(tert-butoxy)-2oxoethyl)phenyl)propanoate (5c)<smiles>CCCCOC(=O)Cc1ccc(C[C@H](NC(F)F)C(=O)OC)cc1</smiles>

General procedure B was followed using $4 \mathbf{c}(52.7 \mathrm{mg}, 0.1 \mathrm{mmol})$ and tert-butyl chloroacetate $(42.9 \mu \mathrm{L}, 0.3 \mathrm{mmol})$. The crude product was purified by flash column chromatography gradient eluting with 0-25\% EtOAc/n-heptane to afford $\mathbf{5 c}$ as a white solid (39.6 mg, $77 \%$ ). ${ }^{1} \mathrm{H}$ NMR $\left(300 \mathrm{MHz}, \mathrm{CDCl}_{3}\right) \delta 7.77$ (dt, J 7.6, $\left.1.0 \mathrm{~Hz}, 2 \mathrm{H}\right), 7.58$ (dd, J 7.8, 4.4 Hz, 2H), 7.41 (tdd, J 7.6, 1.3, 0.6 Hz, 2H), 7.32 (tt, J 7.4, 1.4 Hz, 2H), 7.20 (d, J 7.9 Hz, 2H), 7.05 (d, J 7.7 Hz, 2H), 5.27 $(\mathrm{d}, J 8.3 \mathrm{~Hz}, 1 \mathrm{H}), 4.74-4.61(\mathrm{~m}, 1 \mathrm{H}), 4.50-4.30(\mathrm{~m}, 2 \mathrm{H}), 4.22(\mathrm{t}, J 7.0 \mathrm{~Hz}, 1 \mathrm{H}), 3.73(\mathrm{~s}, 3 \mathrm{H})$, $3.50(\mathrm{~s}, 2 \mathrm{H}), 3.20-3.02(\mathrm{~m}, 2 \mathrm{H}), 1.44(\mathrm{~s}, 9 \mathrm{H}) .{ }^{13} \mathrm{C} \mathrm{NMR}\left(75 \mathrm{MHz}, \mathrm{CDCl}_{3}\right) \delta$ (rotamers $\left.*\right) 172.1$, $171.1,155.7,144.0^{*}, 143.9,141.4,134.4,133.7,129.6,129.5^{*}, 127.8,127.2,125.3 *$, 125.2, 120.1, 120.1, 81.1, 67.1, 54.9, 52.5, 47.3, 42.3, 38.1, 28.2. HRMS m/z: (ESI) ([M+H] $\left.]^{+}\right)$Calcd. for $\mathrm{C}_{31} \mathrm{H}_{33} \mathrm{NO}_{6}$ : 516.2386; Found: 516.2387.

(S)-2-(((9H-Fluoren-9-yl)methoxy)carbonyl)amino)-3-(4-(2-(tert-butoxy)-2oxoethyl)phenyl)propanoic acid (5d)<smiles>CCCCOC(=O)Cc1ccc(C[C@H](NC(=O)OC)C(=O)O)cc1</smiles> 
General procedure B was followed using L- $N_{\alpha}$-Fmoc-Phe(4-iodo)-OH (51.3 mg, $\left.0.1 \mathrm{mmol}\right)$ and tert-butyl chloroacetate $(42.9 \mu \mathrm{L}, 0.3 \mathrm{mmol})$. The crude product was purified by flash column chromatography gradient eluting with 0-25\% EtOAc/n-heptane, then 0-10\% MeOH/DCM and further purified by preparative HPLC to afford $\mathbf{5 d}$ as a white solid (26.6 mg, 53\%). ${ }^{1} \mathrm{H}$ NMR $\left(300 \mathrm{MHz}, \mathrm{CDCl}_{3}\right) \delta 7.76(\mathrm{~d}, J 7.5 \mathrm{~Hz}, 2 \mathrm{H}), 7.55(\mathrm{dt}, J 14.1,6.9 \mathrm{~Hz}, 3 \mathrm{H}), 7.40(\mathrm{t}, J 7.4 \mathrm{~Hz}$, 2H), $7.34-7.29(\mathrm{~m}, 2 \mathrm{H}), 7.19(\mathrm{~d}, J 7.7 \mathrm{~Hz}, 2 \mathrm{H}), 7.10(\mathrm{~d}, J 7.8 \mathrm{~Hz}, 2 \mathrm{H}), 5.29$ (d, J 8.2 Hz, 1H), $4.71(\mathrm{q}, J 6.4 \mathrm{~Hz}, 1 \mathrm{H}), 4.51-4.32(\mathrm{~m}, 2 \mathrm{H}), 4.21(\mathrm{t}, J 7.2 \mathrm{~Hz}, 1 \mathrm{H}), 3.50(\mathrm{~s}, 2 \mathrm{H}), 3.28-3.06$ (m, $2 \mathrm{H}), 1.44(\mathrm{~s}, 9 \mathrm{H}) .{ }^{13} \mathrm{C} \mathrm{NMR}\left(75 \mathrm{MHz}, \mathrm{CDCl}_{3}\right) \delta$ (rotamers*) 175.6, 171.4, 156.1, 143.9*, 143.8, $141.5,134.2,133.7,129.7,129.6^{*}, 127.9,127.2,125.3 *$, 125.2, 120.1, 81.3, 67.3, 54.6, 47.3, 42.3, 37.5, 28.2. HRMS (ESI) m/z: $\left([\mathrm{M}+\mathrm{H}]^{+}\right)$Calcd. for $\mathrm{C}_{30} \mathrm{H}_{32} \mathrm{NO}_{6}$ : 502.2229; Found: 502.2210 .

(S)-Methyl-3-(4-(2-(tert-butoxy)-2-oxoethyl)phenyl)-2-((tertbutoxycarbonyl)amino)propanoate (5e)<smiles>CCCCOC(=O)Cc1ccc(C[C@H](NC(=O)OCc2ccccc2)C(=O)OC)cc1</smiles>

General procedure B was followed using $4 \mathbf{d}(40.5 \mathrm{mg}, 0.1 \mathrm{mmol})$ and tert-butyl chloroacetate $(42.9 \mu \mathrm{L}, 0.3 \mathrm{mmol})$. The crude product was purified by flash column chromatography gradient eluting with 0-25\% EtOAc/n-heptane to afford $\mathbf{5 e}$ as a colourless oil (29.5 mg, $75 \%$ ). ${ }^{1} \mathrm{H}$ NMR $\left(300 \mathrm{MHz}, \mathrm{CDCl}_{3}\right) \delta 7.18(\mathrm{~d}, J 7.8 \mathrm{~Hz}, 2 \mathrm{H}), 7.06(\mathrm{~d}, J 7.8 \mathrm{~Hz}, 2 \mathrm{H}), 4.98(\mathrm{~d}, J 8.3 \mathrm{~Hz}, 1 \mathrm{H}), 4.55$ (q, J 7.1, 6.6 Hz, 1H), 3.69 (s, 3H), 3.48 (s, 2H), 3.07 (dt, $J$ 14.2, 6.7 Hz, 2H), $1.42(\mathrm{~s}, 9 \mathrm{H})$, $1.40(\mathrm{~s}, 9 \mathrm{H}) .{ }^{13} \mathrm{C} \mathrm{NMR}\left(75 \mathrm{MHz}, \mathrm{CDCl}_{3}\right) \delta 172.4,170.9,155.2,134.6,133.5,129.5,129.5$, 80.9, 80.0, 54.5, 52.3, 42.3, 38.0, 28.4, 28.1. HRMS (ESI) m/z: $\left([\mathrm{M}+\mathrm{H}]^{+}\right)$Calcd. for $\mathrm{C}_{21} \mathrm{H}_{32} \mathrm{NO}_{6}$ : 394.2229; Found: 394.2221. 
<smiles>CCCCOC(=O)Cc1ccc(C[C@H](N)C(=O)OC)cc1</smiles>

General procedure B was followed using (S)-methyl-2-amino-3-(4-iodophenyl)propanoate (30.5 mg, $0.1 \mathrm{mmol})$ and tert-butyl chloroacetate $(42.9 \mu \mathrm{L}, 0.3 \mathrm{mmol})$. The crude product was purified by flash column chromatography gradient eluting with $0-25 \%$ EtOAc/n-heptane, then $0-10 \% \mathrm{MeOH} / \mathrm{DCM}$ and further purified by preparative HPLC to afford $\mathbf{5 f}$ as a white solid (13 mg, 45\%). ${ }^{1} \mathrm{H}$ NMR (300 MHz, $\left.\mathrm{CDCl}_{3}\right) \delta 7.22$ - 7.12 (m, 4H), 4.22 (s, 1H), 3.79 (s, 3H), 3.50 (s, 2H), 3.31 (d, $J 13.8 \mathrm{~Hz}, 1 \mathrm{H}), 3.12$ (t, $J 10.3 \mathrm{~Hz}, 1 \mathrm{H}), 1.43(\mathrm{~s}, 9 \mathrm{H}) .{ }^{13} \mathrm{C}$ NMR $(75 \mathrm{MHz}$, $\left.\mathrm{CDCl}_{3}\right) \delta 172.0,169.6,134.1,132.5,130.1,129.9,81.7,54.0,53.3,41.8,36.0,28.2$. HRMS m/z: (ESI) $\left([\mathrm{M}+\mathrm{H}]^{+}\right)$Calcd. for $\mathrm{C}_{16} \mathrm{H}_{24} \mathrm{NO}_{4}$ : 294.1705; Found: 294.1699 .

\section{(2S)-Methyl-2-(((benzyloxy)carbonyl)amino)-3-(4-(1-ethoxy-1-oxopropan-2-}

\section{yl)phenyl)propanoate (7a)}<smiles>CCOC(=O)C(C)c1ccc(C[C@H](NC(=O)OCc2ccccc2)C(=O)OC)cc1</smiles>

General procedure B was followed using $4 \mathbf{a}(43.9 \mathrm{mg}, 0.1 \mathrm{mmol})$ and ethyl 2-chloropropionate $(38.2 \mu \mathrm{L}, 0.3 \mathrm{mmol})$. The crude product was purified by flash column chromatography gradient eluting with $0-25 \%$ EtOAc/n-heptane to afford $7 \mathbf{a}$ as a colourless oil (62.8 mg, $88 \%$ ). ${ }^{1} \mathrm{H}$ NMR $\left(300 \mathrm{MHz}, \mathrm{CDCl}_{3}\right) \delta 7.42-7.25(\mathrm{~m}, 5 \mathrm{H}), 7.21(\mathrm{~d}, J 8.0 \mathrm{~Hz}, 2 \mathrm{H}), 7.04(\mathrm{~d}, J 8.0 \mathrm{~Hz}, 2 \mathrm{H}), 5.21$ $(\mathrm{d}, J 8.3 \mathrm{~Hz}, 1 \mathrm{H}), 5.10(\mathrm{~s}, 2 \mathrm{H}), 4.71-4.59(\mathrm{~m}, 1 \mathrm{H}), 4.22-4.00(\mathrm{~m}, 2 \mathrm{H}), 3.75-3.60(\mathrm{~m}, 4 \mathrm{H})$, $3.16-3.00(\mathrm{~m}, 2 \mathrm{H}), 1.47(\mathrm{~d}, J 7.2 \mathrm{~Hz}, 3 \mathrm{H}), 1.20(\mathrm{t}, J 7.1 \mathrm{~Hz}, 3 \mathrm{H}) .{ }^{13} \mathrm{C} \mathrm{NMR}\left(75 \mathrm{MHz}, \mathrm{CDCl}_{3}\right)$ $\delta 174.6,172.1,155.8,139.7,136.3,134.5,129.6,128.7,128.4,128.2,127.9,67.1,60.9,54.8$, 52.5, 45.3, 37.8, 18.7, 18.7, 14.2. HRMS (ESI) m/z: ([M+H] $\left.]^{+}\right)$Calcd. for $\mathrm{C}_{23} \mathrm{H}_{28} \mathrm{NO}_{6}: 414.1916$; Found: 414.1911. 
<smiles>CCOC(=O)C(F)c1ccc(CC(NC(=O)OCc2ccccc2)C(=O)OC)cc1</smiles>

General procedure B was followed using $4 \mathbf{a}(43.9 \mathrm{mg}, 0.1 \mathrm{mmol})$ and ethyl chlorofluoroacetate $(34.8 \mu \mathrm{L}, 0.3 \mathrm{mmol})$. The crude product was purified by flash column chromatography gradient eluting with $0-25 \%$ EtOAc/n-heptane to afford $7 \mathbf{b}$ as a white solid $(27.1 \mathrm{mg}, 65 \%)$. ${ }^{1} \mathrm{H}$ NMR $\left(300 \mathrm{MHz}, \mathrm{CDCl}_{3}\right) \delta 7.44-7.26(\mathrm{~m}, 7 \mathrm{H}), 7.14(\mathrm{~d}, J 7.9 \mathrm{~Hz}, 2 \mathrm{H}), 5.74\left(\mathrm{~d},{ }^{2} J_{\mathrm{H}-\mathrm{F}} 47.7 \mathrm{~Hz}, 1 \mathrm{H}\right)$, $5.23(\mathrm{~d}, J 8.2 \mathrm{~Hz}, 1 \mathrm{H}), 5.09$ (d, J 12.0 Hz, 1H), 5.05 (d, J $12.0 \mathrm{~Hz}, 1 \mathrm{H}), 4.67$ (q, J $6.5 \mathrm{~Hz}, 1 \mathrm{H})$, $4.35-4.14(\mathrm{~m}, 2 \mathrm{H}), 3.72(\mathrm{~s}, 3 \mathrm{H}), 3.24-3.01(\mathrm{~m}, 2 \mathrm{H}), 1.26(\mathrm{t}, J 7.1 \mathrm{~Hz}, 3 \mathrm{H}) .{ }^{13} \mathrm{C} \mathrm{NMR}(75$ $\left.\mathrm{MHz} \mathrm{CDCl}_{3}\right) \delta 171.9,168.6\left(J_{\mathrm{C}-\mathrm{F}} 27.4 \mathrm{~Hz}\right), 155.7,137.5,136.3,133.3\left(J_{\mathrm{C}-\mathrm{F}} 20.4 \mathrm{~Hz}\right), 129.8$, $128.7,128.4,128.3,127.0\left(J_{\mathrm{C}-\mathrm{F}} 5.9 \mathrm{~Hz}\right), 90.4,88.1,67.2,62.0,54.8,52.6,38.1,14.2 .{ }^{19} \mathrm{~F} \mathrm{NMR}$ $\left(282 \mathrm{MHz}, \mathrm{CDCl}_{3}\right) \delta-179.9\left(\mathrm{~d},{ }^{2} J_{\mathrm{H}-\mathrm{F}} 45.4 \mathrm{~Hz}, 1 \mathrm{~F}\right)$. HRMS (ESI) m/z: $\left([\mathrm{M}+\mathrm{H}]^{+}\right) \mathrm{Calcd}$. for $\mathrm{C}_{22} \mathrm{H}_{25} \mathrm{FNO}_{6}$ : 418.1666; Found: 418.1661.

\section{(S)-Methyl 2-(((benzyloxy)carbonyl)amino)-3-(4}

((methylsulfonyl)methyl)phenyl)propanoate (7c)<smiles>COC(=O)C(Cc1ccc(CS(C)(=O)=O)cc1)NC(=O)OCc1ccccc1</smiles>

General procedure B was followed using 4a (43.9 mg, $0.1 \mathrm{mmol})$ and Chloromethyl methyl sulfone (38.6 mg, $0.3 \mathrm{mmol}$ ). The crude product was purified by flash column chromatography gradient eluting with $0-25 \%$ EtOAc/n-heptane, then $0-10 \% \mathrm{MeOH} / \mathrm{DCM}$ to afford $7 \mathbf{c}$ as a white solid (15.4 mg, 38 \%). ${ }^{1} \mathrm{H}$ NMR (300 MHz, $\left.\mathrm{CDCl}_{3}\right) \delta 7.39-7.30$ (m, 7H), $7.15(\mathrm{~d}, J 7.9 \mathrm{~Hz}$, 2H), $5.24(\mathrm{~d}, J 8.2 \mathrm{~Hz}, 1 \mathrm{H}), 5.08(\mathrm{~s}, 2 \mathrm{H}), 4.66(\mathrm{q}, J 6.2 \mathrm{~Hz}, 1 \mathrm{H}), 4.21(\mathrm{~s}, 2 \mathrm{H}), 3.73(\mathrm{~s}, 3 \mathrm{H}), 3.25$ $-2.99(\mathrm{~m}, 2 \mathrm{H}), 2.73(\mathrm{~s}, 3 \mathrm{H}) .{ }^{13} \mathrm{C} \mathrm{NMR}\left(75 \mathrm{MHz}, \mathrm{CDCl}_{3}\right) \delta 171.9,155.7,137.2,136.3,130.8$, 
130.2, 128.7, 128.4, 128.3, 127.2, 67.18, 6.05, 54.8, 52.6, 42.8, 39.2, 38.2. HRMS (ESI) m/z: $\left([\mathrm{M}+\mathrm{H}]^{+}\right)$Calcd. for $\mathrm{C}_{20} \mathrm{H}_{24} \mathrm{NO}_{6} \mathrm{~S}: 406.1324$; Found: 406.1318 .

(S)-Methyl 2-(((benzyloxy)carbonyl)amino)-3-(4((diethoxyphosphoryl)methyl)phenyl)propanoate (7d)<smiles>CCOP(=O)(Cc1ccc(C[C@H](NC(=O)OCc2ccccc2)C(=O)OCc2ccccc2)cc1)OCC</smiles>

General procedure B was followed using 6 (39.1 $\mathrm{mg}, 0.1 \mathrm{mmol})$ and diethyl chloromethylphosphonate $(46.6 \mu \mathrm{L}, 0.3 \mathrm{mmol})$. The crude product was purified by flash column chromatography gradient eluting with $0-25 \%$ EtOAc/n-heptane, then $0-10 \%$ $\mathrm{MeOH} / \mathrm{DCM}$ to afford $7 \mathbf{d}$ as a colourless oil $(35.7 \mathrm{mg}, 77 \%) .{ }^{1} \mathrm{H} \mathrm{NMR}\left(300 \mathrm{MHz}, \mathrm{CDCl}_{3}\right) \delta$ $7.39-7.27$ (m, 5H), $7.19(\mathrm{dd}, J 8.1,2.5 \mathrm{~Hz}, 2 \mathrm{H}), 7.02(\mathrm{~d}, J 7.8 \mathrm{~Hz}, 2 \mathrm{H}), 5.36(\mathrm{~d}, J 8.2 \mathrm{~Hz}, 1 \mathrm{H})$, $5.06(\mathrm{~s}, 2 \mathrm{H}), 4.60(\mathrm{dt}, J 8.2,5.9 \mathrm{~Hz}, 1 \mathrm{H}), 4.06-3.86(\mathrm{~m}, 4 \mathrm{H}), 3.67(\mathrm{~s}, 3 \mathrm{H}), 3.17-2.93(\mathrm{~m}$, $4 \mathrm{H}), 1.19(\mathrm{t}, J 7.1 \mathrm{~Hz}, 6 \mathrm{H}) .{ }^{13} \mathrm{C} \mathrm{NMR}\left(75 \mathrm{MHz}, \mathrm{CDCl}_{3}\right) \delta 171.9,155.7,136.3,134.5(\mathrm{~d}, J 3.8$ Hz), 130.5 (d, J 9.1 Hz), 130.0 (d, J 6.6 Hz), 129.4 (d, J 3.2 Hz), 128.5, 128.2, 128.1, 66.9, $62.18,62.10,54.9,52.3,37.8,33.4\left(J_{\mathrm{C}-\mathrm{P}} 138.2 \mathrm{~Hz}\right), 16.4,16.3 .{ }^{31} \mathrm{P} \mathrm{NMR}\left(121 \mathrm{MHz}, \mathrm{CDCl}_{3}\right) \delta$ 26.30. HRMS (ESI) m/z: $\left([\mathrm{M}+\mathrm{H}]^{+}\right)$Calcd. for $\mathrm{C}_{23} \mathrm{H}_{31} \mathrm{NO}_{7} \mathrm{P}: 464.1838$; Found: 464.1836.

(2S)-Methyl 2-(((benzyloxy)carbonyl)amino)-3-(4((ethoxy(methyl)phosphoryl)methyl)phenyl)propanoate (7e)<smiles>CCOP(C)(=O)Cc1ccc(C[C@H](NC(=O)OCc2ccccc2)C(=O)OC)cc1</smiles>

General procedure B was followed using 6 (39.1 $\mathrm{mg}, 0.1 \mathrm{mmol})$ and diethyl chloromethylphosphonate $(41 \mu \mathrm{L}, 0.3 \mathrm{mmol})$. The crude product was purified by flash column chromatography gradient eluting with $0-25 \%$ EtOAc/n-heptane, then $0-10 \% \mathrm{MeOH} / \mathrm{DCM}$ to 
afford 7e as a colourless oil (15.2 mg, 35\%). ${ }^{1} \mathrm{H}$ NMR $\left(300 \mathrm{MHz}, \mathrm{CDCl}_{3}\right) \delta 7.42-7.28(\mathrm{~m}$, 5H), 7.18 (dd, J 8.1, 2.2 Hz, 2H), 7.05 (d, J 7.9 Hz, 2H), 5.22 (d, J 8.2 Hz, 1H), 5.09 (s, 2H), $4.64(\mathrm{q}, J 6.4 \mathrm{~Hz}, 1 \mathrm{H}), 4.13-3.92$ (m, 2H), 3.71 (s, 3H), 3.10 (dd, $J$ 16.5, $7.3 \mathrm{~Hz}, 4 \mathrm{H}), 1.44$ $1.20(\mathrm{~m}, 6 \mathrm{H}) .{ }^{13} \mathrm{C} \mathrm{NMR}\left(75 \mathrm{MHz}, \mathrm{CDCl}_{3}\right) \delta 172.0,155.7,136.3,134.7(\mathrm{~d}, J 3.1 \mathrm{~Hz}), 130.9(\mathrm{~d}$, $J 6.0 \mathrm{~Hz}) 130.0(\mathrm{~d}, J 5.5 \mathrm{~Hz}), 129.8$ (d, $J 2.8 \mathrm{~Hz}), 128.7,128.4,128.3,67.2,60.9,60.8,54.9$, 52.5, 38.0, 16.8, 16.7. ${ }^{31} \mathrm{P}$ NMR $\left(121 \mathrm{MHz}, \mathrm{CDCl}_{3}\right) \delta 51.02 . \mathrm{HRMS}(\mathrm{ESI}) \mathrm{m} / \mathrm{z}:\left([\mathrm{M}+\mathrm{H}]^{+}\right) \mathrm{Calcd}$. for $\mathrm{C}_{22} \mathrm{H}_{29} \mathrm{NO}_{6} \mathrm{P}: 434.1732$; Found: 434.1715 .

\section{TFA-Phe(4-iodo)-Thr(OtBu)-OMe (8a)}<smiles>COC(=O)[C@H](NC(=O)[C@H](Cc1ccc(I)cc1)NC(C)C)C(C)OC(C)(C)C</smiles>

To a $100 \mathrm{~mL}$ RBF equipped with a stir bar was added $\mathrm{NH}_{2}-\mathrm{Ser}(\mathrm{O} t \mathrm{Bu})-\mathrm{OMe} \cdot \mathrm{HCl}(1.06 \mathrm{~g}, 5$ mmol), Fmoc-(4-iodo)-Phe-OH (3.08 g, 6 mmol), HATU (2.28 g, 6 mmol), DIPEA (2.63 mL, $30 \mathrm{mmol})$ and DCM $(25 \mathrm{~mL})$. The reaction mixture was stirred overnight at room temperature followed by evaporation of the solvent. 4-Methylpiperidine $(2.96 \mathrm{~mL}, 25 \mathrm{mmol})$ in DCM (25 $\mathrm{mL}$ ) was added and the mixture was stirred for $1 \mathrm{~h}$. The solvent was then evaporated. Methyl trifluoroacetate $(1.51 \mathrm{~mL}, 15 \mathrm{mmol})$, DIPEA $(2.63 \mathrm{~mL}, 15 \mathrm{mmol})$ and $\mathrm{MeOH}(25 \mathrm{~mL})$ were added, and the mixture was stirred overnight. The solvent was evaporated, and the product was purified by flash column chromatography gradient eluting with $0-10 \% \mathrm{MeOH} / \mathrm{DCM}$ to afford 8a as a white solid (1.52 g, 54\%). ${ }^{1} \mathrm{H}$ NMR $\left(300 \mathrm{MHz}, \mathrm{CDCl}_{3}\right) \delta 7.63(\mathrm{~d}, J 6.7 \mathrm{~Hz}, 1 \mathrm{H}), 7.40$ (d, J 7.4 Hz, 1H), $7.05(\mathrm{~d}, J 7.8 \mathrm{~Hz}, 2 \mathrm{H}), 6.27$ (d, J 9.0 Hz, 1H), 4.72 (q, $J 6.7 \mathrm{~Hz}, 1 \mathrm{H}), 4.42$ (dt, J 8.9, 1.7 Hz, 1H), 4.22 (q, J 6.5 Hz, 1H), 3.73 (d, J 1.4 Hz, 3H), $3.20-3.07$ (m, 2H), 1.26 $-0.93(\mathrm{~m}, 12 \mathrm{H}) .{ }^{13} \mathrm{C} \mathrm{NMR}\left(75 \mathrm{MHz}, \mathrm{CDCl}_{3}\right) \delta 170.59,169.34,156.5$ (q, $\left.J_{\mathrm{C}-\mathrm{F}} 37.9 \mathrm{~Hz}\right), 137.98$, 134.97, 131.74, 115.7 (q, $J_{\mathrm{C}-\mathrm{F}} 287.7 \mathrm{~Hz}$ ), 93.29, 74.57, 67.11, 58.34, 54.38, 52.57, 38.16, 28.43, 21.31. ${ }^{19} \mathrm{~F}$ NMR (282 MHz, CDCl3) $\delta$-75.87. HRMS (ESI) $\mathrm{m} / \mathrm{z}:\left([\mathrm{M}+\mathrm{H}]^{+}\right)$Calcd. for $\mathrm{C}_{20} \mathrm{H}_{27} \mathrm{~F}_{3} \mathrm{IN}_{2} \mathrm{O}_{5}$ : 559.0917; Found: 559.0913. 
<smiles>CCCOC(=O)Cc1ccc(C[C@H](NC(=O)C(C)C)C(=O)N[C@H](C(=O)OC)C(C)OC(C)(C)C)cc1</smiles>

General procedure D was followed using 8a $(50.4 \mathrm{mg}, 0.1 \mathrm{mmol})$ and tert-butyl chloroacetate $(42.9 \mu \mathrm{L}, 0.3 \mathrm{mmol})$ as the starting material. The crude product was purified by flash column chromatography gradient eluting with $0-25 \%$ EtOAc/n-heptane, then 0-10\% MeOH/DCM. Then product was purified by preparative HPLC to afford 9a as a white solid (34.6 $\mathrm{mg}, 70 \%$ ). ${ }^{1} \mathrm{H}$ NMR (300 MHz, $\left.\mathrm{CDCl}_{3}\right) \delta 7.34$ (d, J 7.3 Hz, 1H), 7.21 (s, 4H), 6.38 (d, J 9.2 Hz, 1H), 4.75 (q, $J 6.7 \mathrm{~Hz}, 1 \mathrm{H}), 4.45(\mathrm{dd}, J 9.0,1.8 \mathrm{~Hz}, 1 \mathrm{H}), 4.22(\mathrm{qd}, J 6.3,1.8 \mathrm{~Hz}, 1 \mathrm{H}), 3.73(\mathrm{~s}, 3 \mathrm{H}), 3.48$ $(\mathrm{s}, 2 \mathrm{H}), 3.25-3.06(\mathrm{~m}, 2 \mathrm{H}), 1.41(\mathrm{~s}, 9 \mathrm{H}), 1.12(\mathrm{~d}, J 6.3 \mathrm{~Hz}, 3 \mathrm{H}), 1.07(\mathrm{~s}, 9 \mathrm{H}) .{ }^{13} \mathrm{C} \mathrm{NMR}(75$ $\mathrm{MHz}_{\mathrm{CDCl}}$ ) $\delta 170.9,170.7,169.7,156.7$ (q, $\left.J_{\mathrm{C}-\mathrm{F}} 37.7 \mathrm{~Hz}\right), 134.0,133.9,129.8,129.7,115.8$ (q, $\left.J_{\mathrm{C}-\mathrm{F}} 287.5 \mathrm{~Hz}\right), 81.0,74.5,67.2,58.3,54.49,52.48,42.4,38.11,28.4,28.1,21.2 .{ }^{19} \mathrm{~F}$ NMR (282 MHz, CDCl3) $\delta$-75.88. HRMS m/z: (ESI) $\left([\mathrm{M}+\mathrm{H}]^{+}\right)$Calcd. for $\mathrm{C}_{26} \mathrm{H}_{38} \mathrm{~F}_{3} \mathrm{~N}_{2} \mathrm{O}_{7}$ : 547.2631; Found: 547.2630 .

\section{$\operatorname{Ac}-\mathrm{Glu}(\mathrm{O} t \mathrm{Bu})-\mathrm{Phe}(4-\mathrm{iodo})-\mathrm{Thr}(\mathrm{O} t \mathrm{Bu})-\mathrm{NH}_{2}(8 \mathrm{~b})$}<smiles>CCOC(=O)N[C@@H](CCCOC(C)(C)C)C(=O)N[C@@H](Cc1ccc(I)cc1)C(=O)N[C@H](C(N)=O)C(C)OC(C)(C)C</smiles>

General procedure $\mathrm{C}$ was followed using Sieber resin (446 mg, $0.25 \mathrm{mmol}$ ). The crude product was purified by flash column chromatography gradient eluting with $0-10 \% \mathrm{MeOH} / \mathrm{DCM}$ to afford $\mathbf{8 b}$ as a white solid (120.4 mg, 71\%). ${ }^{1} \mathrm{H}$ NMR (300 MHz, DMSO-d6) $\delta 8.07$ (d, $J 8.1$ $\mathrm{Hz}, 1 \mathrm{H}), 7.87$ (d, J 8.1 Hz, 1H), 7.57 (d, J 8.5 Hz, 1H), $7.55-7.45$ (m, 2H), $7.20-7.10$ (m, 1H), $7.04-6.96(\mathrm{~m}, 2 \mathrm{H}), 6.94(\mathrm{~s}, 1 \mathrm{H}), 4.52(\mathrm{td}, J 8.8,4.4 \mathrm{~Hz}, 1 \mathrm{H}), 4.17(\mathrm{td}, J 8.2,5.6 \mathrm{~Hz}, 1 \mathrm{H})$, $4.06(\mathrm{dd}, J 8.5,3.2 \mathrm{~Hz}, 1 \mathrm{H}), 3.94-3.81(\mathrm{~m}, 1 \mathrm{H}), 2.91$ (dd, $J 13.9,4.5 \mathrm{~Hz}, 1 \mathrm{H}), 2.70$ (dd, $J 13.9$, 
$9.4 \mathrm{~Hz}, 1 \mathrm{H}), 2.07(\mathrm{t}, J 8.0 \mathrm{~Hz}, 2 \mathrm{H}), 1.81-1.47(\mathrm{~m}, 5 \mathrm{H}), 1.31(\mathrm{~s}, 9 \mathrm{H}), 1.07$ (s, 9H), 0.91 (d, $J$ $6.2 \mathrm{~Hz}, 3 \mathrm{H}) .{ }^{13} \mathrm{C}$ NMR (75 MHz, DMSO) $\delta 171.6,171.4,171.3,170.5,169.2,137.6,136.7$, $131.8,92.2$, 79.6, 73.6, 66.7, 57.7, 53.5, 51.5, 36.3, 31.3, 28.0, 27.8, 27.5, 22.5, 19.2. HRMS (ESI) m/z: ([M+H] $\left.]^{+}\right)$Calcd. for $\mathrm{C}_{28} \mathrm{H}_{43} \mathrm{IN}_{4} \mathrm{O}_{7}$ : 675.2254; Found: 675.2250.

\section{$\operatorname{Ac}-\mathrm{Glu}(\mathrm{O} t \mathrm{Bu})-p \mathrm{CMF}(\mathrm{O} t \mathrm{Bu})-\mathrm{Thr}(\mathrm{O} t \mathrm{Bu})-\mathrm{NH}_{2}(9 \mathrm{~b})$}<smiles>CCCCOC(=O)Cc1ccc(C[C@H](NC(=O)[C@H](CCC(C)(C)C)NC(=O)OCC)C(=O)N[C@H](C(N)=O)C(C)OC(C)(C)C)cc1</smiles>

General procedure D was followed using $\mathbf{8 b}(67.4 \mathrm{mg}, 0.1 \mathrm{mmol})$ and tert-butyl chloroacetate ( $42.9 \mu \mathrm{L}, 0.3 \mathrm{mmol}$ ) as the starting material. The crude product was purified by flash column chromatography gradient eluting with $0-25 \%$ EtOAc/n-heptane, then 0-10\% MeOH/DCM. Then product was purified by preparative HPLC to afford $\mathbf{9 b}$ as a white solid (43.1 $\mathrm{mg}, 65 \%$ ). ${ }^{1} \mathrm{H}$ NMR (300 MHz, DMSO-d6) $\delta 8.16(\mathrm{~d}, J 8.0 \mathrm{~Hz}, 1 \mathrm{H}), 7.94(\mathrm{~d}, J 8.2 \mathrm{~Hz}, 1 \mathrm{H}), 7.60$ (d, J 8.5 $\mathrm{Hz}, 1 \mathrm{H}), 7.23-7.15(\mathrm{~m}, 3 \mathrm{H}), 7.10$ (d, J 8.2 Hz, 2H), 6.92 (s, 1H), 4.59 (td, J 8.6, $4.7 \mathrm{~Hz}, 1 \mathrm{H})$, 4.25 (td, $J$ 8.2, $5.5 \mathrm{~Hz}, 1 \mathrm{H}), 4.12$ (dd, $J 8.5,3.2 \mathrm{~Hz}, 1 \mathrm{H}), 3.96$ (dd, $J 6.3,3.3 \mathrm{~Hz}, 1 \mathrm{H}), 3.48$ (s, 2H), $3.00(\mathrm{dd}, J 14.0,4.7 \mathrm{~Hz}, 1 \mathrm{H}), 2.81$ (dd, $J 14.0,9.3 \mathrm{~Hz}, 1 \mathrm{H}), 2.15$ (t, J8.0 Hz, 2H), 1.80 (s, $5 \mathrm{H}), 1.38(\mathrm{~d}, J 3.5 \mathrm{~Hz}, 18 \mathrm{H}), 1.13(\mathrm{~s}, 9 \mathrm{H}), 0.97$ (d, J 6.2 Hz, 3H). ${ }^{13} \mathrm{C}$ NMR (75 MHz, DMSOd6) $\delta 171.6,171.4,171.3,170.7,170.5,169.2,136.1,132.6,129.1,128.8,80.0,79.6,73.5,66.7$, 57.7, 53.7, 51.4, 41.3, 36.3, 31.2, 28.0, 27.7, 27.5, 22.4, 19.2. HRMS (ESI) m/z: $\left([\mathrm{M}+\mathrm{H}]^{+}\right)$ Calcd. for $\mathrm{C}_{34} \mathrm{H}_{55} \mathrm{~N}_{4} \mathrm{O} 9$ : 663.3969; Found: 663.3949 . 


\section{Ac-Met-Phe(4-iodo)-Thr(OtBu)- $\mathrm{NH}_{2}(8 c)$}<smiles>CSCC[C@H](NC(=O)Cl)C(=O)NC(Cc1ccc(I)cc1)C(=O)N[C@H](C(N)=O)C(C)OC(C)(C)C</smiles>

General procedure $\mathrm{C}$ was followed using Sieber resin $(446 \mathrm{mg}, 0.25 \mathrm{mmol})$. The crude product was purified by flash column chromatography gradient eluting with $0-10 \% \mathrm{MeOH} / \mathrm{DCM}$ to afford 8c as a white solid (79.1 mg, 51\%). ${ }^{1} \mathrm{H}$ NMR (300 MHz, DMSO-d6) $\delta 8.17$ (d, $J 8.1 \mathrm{~Hz}$, 1H), 7.99 (d, J 8.1 Hz, 1H), 7.59 (dd, J 8.4, 6.6 Hz, 3H), 7.22 (s, 1H), 7.07 (d, J 8.2 Hz, 2H), $7.00(\mathrm{~s}, 1 \mathrm{H}), 4.64-4.51(\mathrm{~m}, 1 \mathrm{H}), 4.37-4.23(\mathrm{~m}, 1 \mathrm{H}), 4.13(\mathrm{dd}, J 8.4,3.2 \mathrm{~Hz}, 1 \mathrm{H}), 3.94(\mathrm{dd}$, $J$ 6.3, $3.4 \mathrm{~Hz}, 1 \mathrm{H}), 2.98$ (dd, $J$ 13.8, $4.4 \mathrm{~Hz}, 1 \mathrm{H}), 2.77$ (dd, $J$ 13.9, $9.7 \mathrm{~Hz}, 1 \mathrm{H}), 2.36$ (t, $J 7.9$ $\mathrm{Hz}, 2 \mathrm{H}), 2.00(\mathrm{~s}, 3 \mathrm{H}), 1.81(\mathrm{~s}, 5 \mathrm{H}), 1.14(\mathrm{~s}, 9 \mathrm{H}), 0.98$ (d, J $6.3 \mathrm{~Hz}, 3 \mathrm{H}) .{ }^{13} \mathrm{C} \mathrm{NMR}(75 \mathrm{MHz}$, DMSO-d $) \delta 171.3,171.3,170.5,169.1,137.6,136.7,131.7,92.1,73.6,66.7,57.6,53.5,51.6$, 36.3, 31.9, 29.4, 28.0, 22.4 19.1, 14.6. HRMS (ESI) m/z: ([M+H] $]^{+}$Calcd. for $\mathrm{C}_{24} \mathrm{H}_{38} \mathrm{IN}_{4} \mathrm{O}_{5} \mathrm{~S}$ : 621.1607; Found: 621.1591.

\section{Ac-Met- $p$ CMF(OtBu)-Thr(OtBu)-NH $(9 c)$}<smiles>CCCCOC(=O)Cc1ccc(C[C@H](NC(=O)[C@H](CCSC)NC(=O)OC)C(=O)N[C@H](C(N)=O)C(C)OC(C)(C)C)cc1</smiles>

General procedure D was followed using 9c $(62 \mathrm{mg}, 0.1 \mathrm{mmol})$ and tert-butyl chloroacetate $(42.9 \mu \mathrm{L}, 0.3 \mathrm{mmol})$ as the starting material. The crude product was purified by flash column chromatography gradient eluting with $0-25 \%$ EtOAc/n-heptane, followed by $0-10 \%$ $\mathrm{MeOH} / \mathrm{DCM}$. Then product was purified by preparative HPLC to afford $\mathbf{9 c}$ as a white solid (26.4 mg, 43 \%). ${ }^{1} \mathrm{H}$ NMR (300 MHz, DMSO-d6) $\delta 8.19$ (d, J 8.0 Hz, 1H), 7.99 (d, J 8.1 Hz, 
1H), 7.57 (d, J 8.4 Hz, 1H), $7.27-7.17(\mathrm{~m}, 3 \mathrm{H}), 7.11(\mathrm{~d}, J 8.1 \mathrm{~Hz}, 2 \mathrm{H}), 6.92(\mathrm{~s}, 1 \mathrm{H}), 4.57$ (td, $J$ 8.8, 4.6 Hz, 1H), $4.32(\mathrm{td}, J 8.2,5.3 \mathrm{~Hz}, 1 \mathrm{H}), 4.12(\mathrm{dd}, J 8.4,3.2 \mathrm{~Hz}, 1 \mathrm{H}), 4.03-3.90$ (m, 1H), 3.50 (under $\mathrm{H}_{2} \mathrm{O}$ peak, 2H), 3.01 (dd, $J$ 14.0, 4.6 Hz, 1H), 2.82 (dd, $J$ 14.1, $9.4 \mathrm{~Hz}, 1 \mathrm{H}$ ), 2.36 (t, J 7.9 Hz, 2H), 2.00 (s, 3H), $1.90-1.60$ (m, 5H), 1.39 (s, 9H), 1.14 (s, 9H), 0.97 (d, J $6.3 \mathrm{~Hz}, 3 \mathrm{H}) .{ }^{13} \mathrm{C}$ NMR (75 MHz, DMSO-d6) $\delta 171.4,170.7,170.4,169.2,136.1,132.6,129.1$, $128.8,80.0,79.4,78.9,78.5,73.6,66.6,57.6,53.8,51.6,41.2,36.3,31.9,29.4,28.0,27.7,22.4$, 19.1, 14.5. HRMS (ESI) m/z: ([M+H] $\left.]^{+}\right)$Calcd. for $\mathrm{C}_{30} \mathrm{H}_{49} \mathrm{~N}_{4} \mathrm{O}_{7} \mathrm{~S}: 609.3322$; Found: 609.3302 .

\section{$\operatorname{Ac}-\mathrm{Tyr}(\mathrm{O} t \mathrm{Bu})-\mathrm{Phe}(4-\mathrm{Iodo})-\mathrm{Thr}(\mathrm{O} t \mathrm{Bu})-\mathrm{NH}_{2}(8 \mathrm{~d})$}<smiles>CC(OC(C)(C)C)C(NC(=O)[C@H](Cc1ccc(I)cc1)NC(=O)[C@H](Cc1ccc(OCC(C)(C)C)cc1)NC(C)(C)C)C(N)=O</smiles>

General procedure $\mathrm{C}$ was followed using Sieber resin $(446 \mathrm{mg}, 0.25 \mathrm{mmol})$. The crude product was purified by flash column chromatography gradient eluting with $0-10 \% \mathrm{MeOH} / \mathrm{DCM}$ to afford 8d as a white solid (112.6 mg, 63\%). ${ }^{1} \mathrm{H}$ NMR (300 MHz, DMSO-d6) $\delta 8.29$ (d, $J 8.1$ $\mathrm{Hz}, 1 \mathrm{H}), 8.00$ (d, J $8.5 \mathrm{~Hz}, 1 \mathrm{H}), 7.60$ (dd, J 10.7, 8.3 Hz, 3H), 7.24 (s, 1H), $7.13-7.04$ (m, $4 \mathrm{H}), 7.00(\mathrm{~s}, 1 \mathrm{H}), 6.87-6.77(\mathrm{~m}, 2 \mathrm{H}), 4.60(\mathrm{td}, J 8.8,4.4 \mathrm{~Hz}, 1 \mathrm{H}), 4.46(\mathrm{td}, J 9.3,4.1 \mathrm{~Hz}, 1 \mathrm{H})$, 4.13 (dd, J 8.4, 3.2 Hz, 1H), 4.03 - 3.91 (m, 1H), 3.01 (dd, J 13.9, 4.5 Hz, 1H), 2.95 - 2.73 (m, 2H), 2.62 (dd, J 14.0, $10.0 \mathrm{~Hz}, 1 \mathrm{H}), 1.71$ (s, 3H), 1.25 (s, 9H), 1.13 (s, 9H), 0.99 (d, J $6.3 \mathrm{~Hz}$, $3 \mathrm{H}) .{ }^{13} \mathrm{C}$ NMR (75 MHz, DMSO- $\left.d_{6}\right) \delta 171.6,171.4,170.5,168.9,153.3,137.6,136.7,132.5$, $131.8,129.6,123.3,92.2$, 77.6, 73.6, 66.7, 57.6, 53.9, 53.6, 36.9, 36.5, 28.6, 28.0, 22.4, 19.2. HRMS (ESI) m/z: $\left([\mathrm{M}+\mathrm{H}]^{+}\right)$Calcd. for $\mathrm{C}_{32} \mathrm{H}_{46} \mathrm{IN}_{4} \mathrm{O}_{6}$ : 709.2462; Found: 709.2459. 


\section{$\operatorname{Ac}-\mathrm{Tyr}(\mathrm{O} t \mathrm{Bu})-p \mathrm{CMF}(\mathrm{O} t \mathrm{Bu})-\mathrm{Thr}(\mathrm{O} t \mathrm{Bu})-\mathrm{NH}_{2}(9 \mathrm{~d})$}<smiles>[CH2-]C(=O)N[C@@H](Cc1ccc(OCC(C)C)cc1)C(=O)NC(Cc1ccc(CC(=O)OCCCC)cc1)C(=O)N[C@H](C(N)=O)[C@H](C)OC(C)(C)C</smiles>

General procedure D was followed using $8 \mathbf{d}(70.8 \mathrm{mg}, 0.1 \mathrm{mmol})$ and tert-butyl chloroacetate $(42.9 \mu \mathrm{L}, 0.3 \mathrm{mmol})$ as the starting material. The crude product was purified by flash column chromatography gradient eluting with $0-25 \%$ EtOAc/n-heptane, then 0-10\% MeOH/DCM. Then product was further purified by preparative HPLC to afford $9 d$ as a white solid $(43.2 \mathrm{mg}$, $62 \%) .{ }^{1} \mathrm{H}$ NMR (300 MHz, DMSO-d 6$) \delta 8.32(\mathrm{~d}, J 7.9 \mathrm{~Hz}, 1 \mathrm{H}), 8.00(\mathrm{~d}, J 8.5 \mathrm{~Hz}, 1 \mathrm{H}), 7.59$ $(\mathrm{d}, J 8.3 \mathrm{~Hz}, 1 \mathrm{H}), 7.25-7.18(\mathrm{~m}, 3 \mathrm{H}), 7.16-7.05(\mathrm{~m}, 4 \mathrm{H}), 6.92(\mathrm{~s}, 1 \mathrm{H}), 6.86-6.76(\mathrm{~m}, 2 \mathrm{H})$, 4.53 (dtd, $J 34.7,9.0,4.2 \mathrm{~Hz}, 2 \mathrm{H}), 4.13$ (dd, $J 8.4,3.1 \mathrm{~Hz}, 1 \mathrm{H}), 4.05-3.92$ (m, 1H), 3.49 (s, 2H), $3.03(\mathrm{dd}, J 14.1,4.6 \mathrm{~Hz}, 1 \mathrm{H}), 2.87$ (td, $J$ 15.1, 14.0, $6.7 \mathrm{~Hz}, 2 \mathrm{H}), 2.63(\mathrm{dd}, J 14.0,10.1$ $\mathrm{Hz}, 1 \mathrm{H}), 1.70(\mathrm{~s}, 3 \mathrm{H}), 1.38(\mathrm{~s}, 9 \mathrm{H}), 1.25$ (s, 9H), 1.13 (s, 9H), 0.99 (d, J 6.2 Hz, 3H). ${ }^{13} \mathrm{C}$ NMR (75 MHz, DMSO-d6) $\delta 171.6,171.4,170.8,170.5,168.9,153.3,136.1,132.6,132.6,129.6$, 129.2 , 128.8, 123.2, 80.0, 77.5, 73.6, 66.7, 57.6, 53.9, 53.7, 41.3, 36.9, 36.5, 28.5, 28.0, 27.7, 22.4, 19.1. HRMS (ESI) m/z: $\left([\mathrm{M}+\mathrm{H}]^{+}\right)$Calcd. for $\mathrm{C}_{38} \mathrm{H}_{57} \mathrm{~N}_{4} \mathrm{O}_{8}$ : 697.4176; Found: 697.4185.

\section{$\operatorname{Ac}-\operatorname{Trp}(\mathrm{Boc})-\mathrm{Phe}(4-\mathrm{iod})$ )-Thr(OtBu)-NH$(8 \mathrm{e})$}<smiles>CC(C)CO[C@H](NC(=O)[C@H](Cc1ccc(I)cc1)NC(=O)[C@H](Cc1cn(C(=O)OC(C)(C)C)c2ccccc12)NC(=O)OCc1ccccc1)C(N)=O</smiles>

General procedure $\mathrm{C}$ was followed using Sieber resin (446 mg, $0.25 \mathrm{mmol}$ ). The crude product was purified by flash column chromatography gradient eluting with $0-10 \% \mathrm{MeOH} / \mathrm{DCM}$ to 
afford 8e a white solid (132.3 mg, 68\%). ${ }^{1} \mathrm{H}$ NMR (300 MHz, DMSO- $\left.d_{6}\right) \delta 8.39$ (d, $J 8.1 \mathrm{~Hz}$, $1 \mathrm{H}), 8.13(\mathrm{~d}, J 8.5 \mathrm{~Hz}, 1 \mathrm{H}), 8.00(\mathrm{~d}, J 8.1 \mathrm{~Hz}, 1 \mathrm{H}), 7.67$ (d, J 7.7 Hz, 1H), 7-60-7.56 (m, 3H), 7.48 (s, 1H), 7.34-7.21 (m, 3H), $7.11-7.04(\mathrm{~m}, 2 \mathrm{H}), 7.02$ (s, 1H), 4.59 (dt, J 8.7, 4.6 Hz, 2H), 4.13 (dd, $J$ 8.3, $3.2 \mathrm{~Hz}, 1 \mathrm{H}), 3.96$ (dd, J 6.3, $3.2 \mathrm{~Hz}, 1 \mathrm{H}), 3.02$ (ddd, J 14.2, 9.0, $4.2 \mathrm{~Hz}, 2 \mathrm{H}$ ), 2.80 (dd, $J$ 14.5, $9.8 \mathrm{~Hz}, 2 \mathrm{H}), 1.73$ (s, 3H), 1.62 (s, 9H), 1.12 (s, 9H), 0.99 (d, J 6.2 Hz, 3H). ${ }^{13} \mathrm{C}$ NMR (75 MHz, DMSO-d6) $\delta 171.5,171.4,170.4,169.1,149.0,137.6,136.7,134.6,131.7$, $130.3,124.3,123.8,122.4,119.4,116.7,114.6,92.2,83.5,73.6,66.7,57.6,52.1,36.3,28.0$, 27.7, 27.3, 22.4, 19.2. Note: 1 carbon signal not observed. HRMS (ESI) m/z: $\left([\mathrm{M}+\mathrm{H}]^{+}\right) \mathrm{Calcd}$. for: $\mathrm{C}_{35} \mathrm{H}_{47} \mathrm{IN}_{5} \mathrm{O}_{7}$ : 776.2520; Found: 776.2521 .

\section{$\operatorname{Ac}-\operatorname{Trp}(\mathrm{Boc})-p \mathrm{CMF}(\mathrm{O} t \mathrm{Bu})-\mathrm{Thr}(\mathrm{O} t \mathrm{Bu})-\mathrm{NH}_{2}(9 \mathrm{e})$}<smiles>CCCCOC(=O)Cc1ccc(C[C@H](NC(=O)[C@H](Cc2cn(C(=O)OC(C)(C)C)c3ccccc23)NC(=O)OC(C)(C)C)[C@H](NC(=O)[C@H](C)OC(C)C)C(N)=O)cc1</smiles>

General procedure D was followed using $8 \mathbf{e}(77.5 \mathrm{mg}, 0.1 \mathrm{mmol})$ and tert-butyl chloroacetate (42.9 $\mu \mathrm{L}, 0.3 \mathrm{mmol}$ ) as the starting material. The crude product was purified by flash column chromatography gradient eluting with $0-25 \%$ EtOAc/n-heptane, then $0-10 \% \mathrm{MeOH} / \mathrm{DCM}$. Then product was further purified by preparative HPLC to afford $9 \mathbf{e}$ as a white solid $(39.5 \mathrm{mg}$, $52 \%) .{ }^{1} \mathrm{H}$ NMR (300 MHz, DMSO-d 6 ) $\delta 8.41(\mathrm{~d}, J 7.9 \mathrm{~Hz}, 1 \mathrm{H}), 8.14(\mathrm{~d}, J 8.5 \mathrm{~Hz}, 1 \mathrm{H}), 8.00$ $(\mathrm{d}, J 8.2 \mathrm{~Hz}, 1 \mathrm{H}), 7.68(\mathrm{~d}, J 7.8 \mathrm{~Hz}, 1 \mathrm{H}), 7.56(\mathrm{~d}, J 8.3 \mathrm{~Hz}, 1 \mathrm{H}), 7.49$ (s, 1H), $7.36-7.16$ (m, $5 \mathrm{H}), 7.12(\mathrm{~d}, J 8.0 \mathrm{~Hz}, 2 \mathrm{H}), 6.94(\mathrm{~s}, 1 \mathrm{H}), 4.68-4.54(\mathrm{~m}, 2 \mathrm{H}), 4.13(\mathrm{dd}, J 8.3,3.1 \mathrm{~Hz}, 1 \mathrm{H}), 3.97$ (dd, $J$ 6.3, $3.2 \mathrm{~Hz}, 1 \mathrm{H}), 3.48$ (s, 2H), 3.04 (td, $J$ 12.8, 11.1, $4.3 \mathrm{~Hz}, 2 \mathrm{H}), 2.83$ (td, $J$ 15.4, 14.9, $9.7 \mathrm{~Hz}, 2 \mathrm{H}), 1.72(\mathrm{~s}, 3 \mathrm{H}), 1.61(\mathrm{~s}, 9 \mathrm{H}), 1.37(\mathrm{~s}, 9 \mathrm{H}), 1.12(\mathrm{~s}, 9 \mathrm{H}), 0.99(\mathrm{~d}, J 6.2 \mathrm{~Hz}, 3 \mathrm{H}) .{ }^{13} \mathrm{C}$ NMR (75 MHz, DMSO-d6) $\delta 171.6,171.4,170.6,170.4,169.1,149.0,142.6,136.1,134.6$, 132.6, 130.3, 129.1, 128.8, 124.2, 123.8, 122.3, 119.4, 116.7, 114.6, 83.5, 80.0, 73.6, 66.7, 57.6, 54.1, 52.2, 41.2, 36.4, 28.0, 27.7, 27.4, 22.4, 19.1. HRMS (ESI) m/z: ([M+H] $\left.]^{+}\right)$Calcd. for $\mathrm{C}_{41} \mathrm{H}_{58} \mathrm{~N}_{5} \mathrm{O}_{9}$ : 764.4234; Found: 764.4217. 
<smiles>CC(C)O[C@H](C)[C@H](NC(=O)[C@H](Cc1ccc(I)cc1)NC(=O)[C@H](CCCNC(=N)NCc1ccccc1)NC(=O)Cc1ccccc1)C(N)=O</smiles>

General procedure $\mathrm{C}$ was followed using Sieber resin (446 mg, $0.25 \mathrm{mmol}$ ). The crude product was purified by flash column chromatography gradient eluting with $0-10 \% \mathrm{MeOH} / \mathrm{DCM}$ to afford 8f as a white solid (152.4 mg, 68\%). ${ }^{1} \mathrm{H}$ NMR (300 MHz, DMSO-d $) \delta 8.16$ (d, $J 8.1$ Hz, 1H), 7.93 (d, J 8.3 Hz, 1H), $7.63-7.47$ (m, 3H), 7.24 (s, 1H), $7.11-6.96$ (m, 3H), 6.63 (s, 1H), 6.40 (s, 2H), 4.56 (td, J 8.8, $4.7 \mathrm{~Hz}, 1 \mathrm{H}), 4.21$ (t, J 7.2 Hz, 1H), 4.12 (dd, J 8.4, $3.3 \mathrm{~Hz}$, 1H), $3.99-3.86(\mathrm{~m}, 1 \mathrm{H}), 2.97$ (d, J 11.4 Hz, 5H), 2.76 (dd, J 13.9, $9.3 \mathrm{~Hz}, 1 \mathrm{H}), 2.47$ (s, 3H), $2.42(\mathrm{~s}, 3 \mathrm{H}), 2.00(\mathrm{~s}, 3 \mathrm{H}), 1.80(\mathrm{~s}, 3 \mathrm{H}), 1.62-1.47(\mathrm{~m}, 1 \mathrm{H}), 1.40(\mathrm{~s}, 9 \mathrm{H}), 1.12(\mathrm{~s}, 9 \mathrm{H}), 0.96(\mathrm{~d}$, $J 6.3 \mathrm{~Hz}, 3 \mathrm{H}) .{ }^{13} \mathrm{C} \mathrm{NMR}(75 \mathrm{MHz}$, DMSO-d6) $\delta 171.7,171.4,170.5,169.1,157.5,156.0,137.5$, $137.3,136.7,134.2,131.7,131.5,124.3,116.3,92.1,86.3,73.6,66.7,57.6,53.6,51.9,42.5$, 36.4, 29.4, 28.3, 28.0 (2x C), 25.4, 22.5, 19.1, 19.0, 17.6, 12.3. HRMS (ESI) m/z: $\left([\mathrm{M}+\mathrm{H}]^{+}\right)$ Calcd. for $\mathrm{C}_{38} \mathrm{H}_{57} \mathrm{IN}_{7} \mathrm{O}_{8} \mathrm{~S}$ : 898.3034; Found: 898.3052.

\section{$\operatorname{Ac}-\operatorname{Arg}(\mathrm{Pbf})-p \mathrm{CMF}(\mathrm{O} t \mathrm{Bu})-\mathrm{Thr}(\mathrm{O} t \mathrm{Bu})-\mathrm{NH}_{2}(9 \mathrm{f})$}<smiles>CCCCOC(=O)Cc1ccc(C[C@H](NC(=O)[C@H](CCCNC(=N)NCc2ccccc2)NC(=O)NCc2ccccc2)C(=O)N[C@H](C(N)=O)[C@@H](C)OC(C)C)cc1</smiles> 
General procedure D was followed using $\mathbf{8 f}(89.8 \mathrm{mg}, 0.1 \mathrm{mmol})$ and tert-butyl chloroacetate $(42.9 \mu \mathrm{L}, 0.3 \mathrm{mmol})$ as the starting material. The crude product was purified by flash column chromatography gradient eluting with $0-25 \%$ EtOAc/n-heptane, then 0-10\% MeOH/DCM. Then product was further purified by preparative HPLC to afford $9 f$ as a white solid $(43.5 \mathrm{mg}$, 49\%). ${ }^{1} \mathrm{H}$ NMR (300 MHz, DMSO-d6) $\delta 8.18(\mathrm{~d}, J 7.9 \mathrm{~Hz}, 1 \mathrm{H}), 7.93$ (d, J 8.2 Hz, 1H), 7.53 (d, $J 8.4 \mathrm{~Hz}, 1 \mathrm{H}), 7.26-7.14(\mathrm{~m}, 3 \mathrm{H}), 7.10(\mathrm{~d}, J 8.1 \mathrm{~Hz}, 2 \mathrm{H}), 6.93(\mathrm{~s}, 1 \mathrm{H}), 6.63(\mathrm{~s}, 1 \mathrm{H}), 6.40(\mathrm{~s}$, 1H), $4.62-4.49$ (m, 1H), 4.12 (dd, J 8.3, 3.2 Hz, 2H), 3.93 (dd, J 6.3, 3.3 Hz, 1H), 3.48 (s, 2H), 2.97 (d, J 8.9 Hz, 5H), 2.82 (dd, J 14.0, $9.3 \mathrm{~Hz}, 1 \mathrm{H}), 2.47$ (s, 3H), 2.42 (s, 3H), 2.00 (s, $3 \mathrm{H}), 1.80(\mathrm{~s}, 3 \mathrm{H}), 1.63-1.29(\mathrm{~m}, 19 \mathrm{H}), 1.12(\mathrm{~s}, 9 \mathrm{H}), 0.96(\mathrm{~d}, J 6.2 \mathrm{~Hz}, 3 \mathrm{H}) .{ }^{13} \mathrm{C} \mathrm{NMR}(75$ MHz, DMSO-d6) $\delta 171.7,171.4,170.7,170.5,169.1,157.5,156.1,137.3,136.1,134.2,132.6$, $131.5,129.1,128.8,124.3,117.3,116.3,86.3,80.1$, 73.6, 66.7, 57.6, 53.9, 51.9, 42.5, 41.2, 36.4, 29.5, 28.3, 28.0, 27.7, 25.3, 22.4, 19.1, 18.9, 17.6, 12.3. HRMS (ESI) m/z: $\left([\mathrm{M}+\mathrm{H}]^{+}\right)$ Calcd. for $\mathrm{C}_{44} \mathrm{H}_{68} \mathrm{~N}_{7} \mathrm{O}_{10} \mathrm{~S}: 886.4748$; Found: 886.4762 .

\section{Ac-Glu(OtBu)-Phe(4-bromo)-Thr(OtBu)-NH2 (8g)}<smiles>CCOC(=O)N[C@@H](CCCOC(C)(C)C)C(=O)N[C@@H](Cc1ccc(Br)cc1)C(=O)N[C@H](C(N)=O)C(C)(C)C</smiles>

General procedure $\mathrm{C}$ was followed using Sieber resin (446 mg, $0.25 \mathrm{mmol}$ ). The crude product was purified by flash column chromatography gradient eluting with $0-10 \% \mathrm{MeOH} / \mathrm{DCM}$ to afford $8 \mathrm{~g}$ as a white solid (103.1 mg, 66\%). ${ }^{1} \mathrm{H}$ NMR (300 MHz, DMSO-d6) $\delta 8.14(\mathrm{~d}, J 8.1$ Hz, 1H), 7.94 (d, J 8.1 Hz, 1H), 7.64 (d, J 8.5 Hz, 1H), 7.41 (d, J 8.4 Hz, 2H), 7.20 (d, J 8.4 $\mathrm{Hz}, 3 \mathrm{H}), 7.00$ (s, 1H), 4.60 (td, J 8.9, $4.5 \mathrm{~Hz}, 1 \mathrm{H}), 4.31-4.17(\mathrm{~m}, 1 \mathrm{H}), 4.12$ (dd, J 8.5, $3.2 \mathrm{~Hz}$, 1H), $4.00-3.89$ (m, 1H), 2.99 (dd, J 13.9, 4.6 Hz, 1H), 2.78 (dd, J 13.9, 9.5 Hz, 1H), 2.14 (t, $J 8.0 \mathrm{~Hz}, 2 \mathrm{H}), 1.86-1.55(\mathrm{~m}, 5 \mathrm{H}), 1.38(\mathrm{~s}, 9 \mathrm{H}), 1.13(\mathrm{~s}, 9 \mathrm{H}), 0.98(\mathrm{~d}, J 6.3 \mathrm{~Hz}, 3 \mathrm{H}) .{ }^{13} \mathrm{C} \mathrm{NMR}$ (75 MHz, DMSO-d6) $\delta 171.6,171.4,171.3,170.5$, 169.2, 137.2, 131.5, 130.8, 119.4, 79.6, 73.5, 66.7, 57.7, 53.5, 51.5, 36.2, 31.2, 28.0, 27.7, 27.5, 22.4, 19.2. HRMS (ESI) m/z: $\left([\mathrm{M}+\mathrm{H}]^{+}\right)$ Calcd. for $\mathrm{C}_{28} \mathrm{H}_{44}{ }^{79} \mathrm{BrN}_{4} \mathrm{O}_{7}$ : 627.2393; Found: 627.2387. 


\section{$\operatorname{Ac-Glu}(\mathrm{O} t \mathrm{Bu})-\mathrm{Pmp}\left(\mathrm{OEt}_{2}\right)-\mathrm{Thr}(\mathrm{O} t \mathrm{Bu})(9 \mathrm{~g})$}<smiles>CCOC(=O)CC[C@H](NC(=O)OCC)C(=O)N[C@@H](Cc1ccc(CP(=O)(OCC)OCC(C)(C)C)cc1)C(=O)N[C@@H](C(N)=O)C(C)OC(C)(C)C</smiles>

General procedure D was followed using $\mathbf{8 g}(62.6 \mathrm{mg}, 0.1 \mathrm{mmol})$ and diethyl chloromethylphosphonate $(46.6 \mu \mathrm{L}, 0.3 \mathrm{mmol})$ with the addition of water $(10$ eq.). The crude product was purified by flash column chromatography gradient eluting with $0-25 \%$ EtOAc/nheptane, then $0-10 \% \mathrm{MeOH} / \mathrm{DCM}$. Then product was further purified by preparative HPLC to afford $9 \mathrm{~g}$ as a white solid (26.7 mg, $38 \%$ ). ${ }^{1} \mathrm{H}$ NMR (300 MHz, DMSO-d6) $\delta 8.17$ (d, $J 7.9 \mathrm{~Hz}$, 1H), 7.94 (d, J 8.1 Hz, 1H), 7.61 (d, J 8.5 Hz, 1H), $7.23-7.08$ (m, 5H), $6.84-6.78$ (m, 1H), $4.67-4.53(\mathrm{~m}, 1 \mathrm{H}), 4.32-4.21(\mathrm{~m}, 1 \mathrm{H}), 4.10(\mathrm{dd}, J 8.6,3.1 \mathrm{~Hz}, 1 \mathrm{H}), 4.00-3.80(\mathrm{~m}, 5 \mathrm{H})$, $3.18(\mathrm{~s}, 1 \mathrm{H}), 3.11(\mathrm{~s}, 1 \mathrm{H}), 2.98(\mathrm{dd}, J 14.1,5.1 \mathrm{~Hz}, 1 \mathrm{H}), 2.80(\mathrm{dd}, J 13.9,9.1 \mathrm{~Hz}, 1 \mathrm{H}), 2.15(\mathrm{t}$, $J 8.0 \mathrm{~Hz}, 2 \mathrm{H}), 1.87-1.55(\mathrm{~m}, 5 \mathrm{H}), 1.37$ (s, 9H), $1.20-1.10(\mathrm{~m}, 15 \mathrm{H}), 0.97$ (d, J $6.3 \mathrm{~Hz}, 3 \mathrm{H})$. ${ }^{13} \mathrm{C}$ NMR (75 MHz, DMSO-d6) $\delta$ 171.6, 171.4, 171.2, 170.7, 169.1, 135.9 (d, J 3.9 Hz), 130 (d, J 8.9 Hz), 129.4 (d, J 6.4 Hz), 129.1 (d, J 2.3 Hz), 79.6, 73.5, 66.6, 61.4, 61.3, 57.7, 53.7, 51.4, 36.4, 31.2, 28.0, 27.7, 27.6, 22.4, 19.2, 16.2, 16.1. ${ }^{31} \mathrm{P}$ NMR (121 MHz, DMSO-d6) $\delta$ 26.69. HRMS m/z: (ESI) $\left([\mathrm{M}+\mathrm{H}]^{+}\right)$Calcd. for $\mathrm{C}_{33} \mathrm{H}_{56} \mathrm{~N}_{4} \mathrm{O}{ }_{10} \mathrm{P}: 699.3734$; Found: 699.3727.

\section{Ac-Glu-Phe(4-iodo)-Thr-NH 2 (10a)}<smiles>CC(=O)N[C@@H](CCC(=O)O)C(=O)N[C@@H](Cc1ccc(I)cc1)C(=O)N[C@H](C(N)=O)[C@@H](C)O</smiles>

General procedure $\mathrm{C}$ was followed using Rink amide resin (316 $\mathrm{mg}, 0.25 \mathrm{mmol})$. The crude product was purified by preparative HPLC to afford $\mathbf{1 0 a}$ as a white solid $(82.2 \mathrm{mg}, 58 \%) .{ }^{1} \mathrm{H}$ 
NMR (300 MHz, DMSO-d6) $\delta 8.05(\mathrm{~d}, J 8.1 \mathrm{~Hz}, 1 \mathrm{H}), 8.00(\mathrm{~d}, J 7.7 \mathrm{~Hz}, 1 \mathrm{H}), 7.77(\mathrm{~d}, J 8.6 \mathrm{~Hz}$, 1H), 7.57 (d, J 8.2 Hz, 2H), $7.12-7.03(\mathrm{~m}, 4 \mathrm{H}), 4.88(\mathrm{~s}, 1 \mathrm{H}), 4.56$ (t, J 10.8 Hz, 1H), 4.18 (q, $J 7.5 \mathrm{~Hz}, 1 \mathrm{H}), 4.12-3.95$ (m, 2H), 3.02 (dd, $J 14.0,4.2 \mathrm{~Hz}, 1 \mathrm{H}), 2.77$ (dd, $J 14.1,10.1 \mathrm{~Hz}$, 1H), $2.15(\mathrm{t}, J 7.9 \mathrm{~Hz}, 2 \mathrm{H}), 1.81(\mathrm{~s}, 6 \mathrm{H}), 1.01$ (d, $J 6.3 \mathrm{~Hz}, 3 \mathrm{H}) .{ }^{13} \mathrm{C}$ NMR (75 MHz, DMSOd6) $\delta 173.9,172.0,171.3,170.8,169.5,137.7,136.7,131.8,92.1,66.3,58.2,53.6,51.9,36.4$, 30.1, 27.2, 22.4, 20.1. HRMS (ESI) m/z: $\left([\mathrm{M}+\mathrm{H}]^{+}\right)$Calcd. for $\mathrm{C}_{20} \mathrm{H}_{28} \mathrm{IN}_{4} \mathrm{O}_{7}$ : 563.1002; Found: 563.0995 .

Ac-Glu- $p$ CMF(OtBu)-Thr-NH2 (11a)<smiles>CCCCOC(=O)Cc1ccc(C[C@H](NC(=O)[C@H](CCC(=O)O)NC(=O)O)C(=O)N[C@H](C(N)=O)[C@H](C)O)cc1</smiles>

General procedure D was followed using $10 \mathrm{a}(56.2 \mathrm{mg}, 0.1 \mathrm{mmol})$ and tert-butyl chloroacetate $(42.9 \mu \mathrm{L}, 0.3 \mathrm{mmol})$ as the starting material. The crude product was subjected to the Strong Cation Exchange column (SCX) to remove residual HE and then the product was eluted with $\mathrm{MeCN}$. The crude was then purified by preparative HPLC to afford 11a as a white solid (30.1 mg, 45\%). ${ }^{1} \mathrm{H}$ NMR (300 MHz, DMSO- $\left.d_{6}\right) \delta 12.01$ (s, 1H), 8.04 (dd, $J 14.6,7.8$ Hz, 2H), 7.73 (d, J 8.6 Hz, 1H), 7.19 (d, J 8.1 Hz, 2H), 7.11 (d, J8.1 Hz, 2H), 7.06 (s, 1H), 6.94 (s, 1H), 4.88 $(\mathrm{s}, 1 \mathrm{H}), 4.56(\mathrm{td}, J 9.0,8.3,4.5 \mathrm{~Hz}, 1 \mathrm{H}), 4.23-4.00(\mathrm{~m}, 3 \mathrm{H}), 3.48(\mathrm{~s}, 2 \mathrm{H}), 3.04(\mathrm{dd}, J 13.9,4.5$ $\mathrm{Hz}, 1 \mathrm{H}), 2.82(\mathrm{dd}, J 14.0,9.6 \mathrm{~Hz}, 1 \mathrm{H}), 2.13(\mathrm{t}, J 7.9 \mathrm{~Hz}, 2 \mathrm{H}), 1.91-1.58$ (m, 5H), 1.39 (s, 9H), $1.01(\mathrm{~d}, J 6.2 \mathrm{~Hz}, 3 \mathrm{H}) .{ }^{13} \mathrm{C} \mathrm{NMR}\left(75 \mathrm{MHz}, \mathrm{DMSO}-d_{6}\right) \delta 174.1,172.1,171.4,171.1,170.5$, 169.6, 136.2, 132.6, 129.2, 128.9, 80.1, 66.3, 58.2, 53.9, 52.0, 41.3, 36.4, 30.1, 27.7, 27.3, 22.4, 20.1. HRMS (ESI) m/z: ([M+H] $\left.]^{+}\right)$Calcd. for $\mathrm{C}_{26} \mathrm{H}_{39} \mathrm{~N}_{4} \mathrm{O}_{9}$ : 551.2717; Found: 551.2702. 
<smiles>C[C@H](O)[C@H](NC(=O)[C@H](Cc1ccc(I)cc1)NC(=O)[C@H](Cc1c[nH]c2ccccc12)NC(=O)[O-])C(N)=O</smiles>

General procedure $\mathrm{C}$ was followed using Rink amide resin $(316 \mathrm{mg}, 0.25 \mathrm{mmol})$. The crude product was purified by preparative HPLC to afford $10 \mathrm{~b}$ as a white solid $(50.5 \mathrm{mg}, 52 \%) .{ }^{1} \mathrm{H}$ NMR (300 MHz, DMSO-d6) $\delta 10.75(\mathrm{~d}, J 2.4 \mathrm{~Hz}, 1 \mathrm{H}), 8.22$ (d, J 8.0 Hz, 1H), 8.04 (d, $J 8.0$ $\mathrm{Hz}, 1 \mathrm{H}), 7.73$ (d, J 8.6 Hz, 1H), 7.57 (t, J 7.6 Hz, 3H), 7.31 (d, J 8.0 Hz, 1H), $7.19-6.90$ (m, $7 \mathrm{H}), 4.91(\mathrm{~s}, 1 \mathrm{H}), 4.65-4.40(\mathrm{~m}, 2 \mathrm{H}), 4.18-3.96(\mathrm{~m}, 2 \mathrm{H}), 3.12-2.95(\mathrm{~m}, 2 \mathrm{H}), 2.89-2.75$ (m, 2H), $1.74(\mathrm{~s}, 3 \mathrm{H}), 1.03$ (d, J $6.3 \mathrm{~Hz}, 3 \mathrm{H}) .{ }^{13} \mathrm{C}$ NMR (75 MHz, DMSO-d6) $\delta$ 172.0, 171.9, 170.7, 169.3, 137.7, 136.7, 136.0, 131.8, 127.3, 123.4, 120.8, 118.4, 118.2, 111.3, 110.2, 92.1, 66.4, 58.1, 53.8, 53.3, 36.3, 27.5, 22.5, 20.0. HRMS (ESI) m/z: $\left([\mathrm{M}+\mathrm{H}]^{+}\right)$Calcd. for $\mathrm{C}_{26} \mathrm{H}_{31} \mathrm{IN}_{5} \mathrm{O}_{5}$ : 620.1370; Found: 620.1371.

\section{Ac-Trp- $p$ CMF(OtBu)-Thr-NH2 (11b)}<smiles>[CH2]C(=O)N[C@@H](Cc1c[nH]c2ccccc12)C(=O)N[C@@H](Cc1ccc(CC(=O)OCCCC)cc1)NC(C(N)=O)[C@H](C)O</smiles>

General procedure D was followed using $10 \mathrm{~b}(62 \mathrm{mg}, 0.1 \mathrm{mmol})$ and tert-butyl chloroacetate $(42.9 \mu \mathrm{L}, 0.3 \mathrm{mmol})$ as the starting material. The crude product was subjected to the Strong Cation Exchange column (SCX) to remove residual HE and then the product was eluted with $\mathrm{MeCN}$. The crude was then purified by preparative HPLC to afford 11b as a white solid (20 mg, 33\%). ${ }^{1} \mathrm{H}$ NMR (300 MHz, DMSO-d6) $\delta 10.72$ (d, $\left.J 2.4 \mathrm{~Hz}, 1 \mathrm{H}\right), 8.21$ (d, $\left.J 7.8 \mathrm{~Hz}, 1 \mathrm{H}\right)$, 
$8.02(\mathrm{~d}, J 7.9 \mathrm{~Hz}, 1 \mathrm{H}), 7.68(\mathrm{~d}, J 8.5 \mathrm{~Hz}, 1 \mathrm{H}), 7.56(\mathrm{~d}, J 7.7 \mathrm{~Hz}, 1 \mathrm{H}), 7.31(\mathrm{~d}, J 8.0 \mathrm{~Hz}, 1 \mathrm{H})$, $7.20(\mathrm{~d}, J 8.0 \mathrm{~Hz}, 2 \mathrm{H}), 7.12(\mathrm{~d}, J 8.1 \mathrm{~Hz}, 2 \mathrm{H}), 7.08-6.92(\mathrm{~m}, 5 \mathrm{H}), 4.63-4.41(\mathrm{~m}, 2 \mathrm{H}), 4.08$ (ddd, $J$ 16.3, 7.4, 3.4 Hz, 2H), 3.49 (s, 2H), $3.07-2.99$ (m, 2H), $2.92-2.80$ (m, 2H), 1.73 (s, $3 \mathrm{H}), 1.38$ (s, 9H), 1.03 (d, J 6.3 Hz, 3H) (one -OH proton not observed). ${ }^{13} \mathrm{C}$ NMR (75 MHz, DMSO) $\delta 172.1,172.0,171.0,170.5,169.4,136.2,136.1,132.6,129.2,128.9,127.3,123.5$, $120.8,118.4,118.2$, 111.3, 110.2, 80.1, 66.4, 58.1, 54.2, 53.4, 41.3, 36.4, 27.7, 27.5, 22.5, 20.0. HRMS (ESI) m/z: $\left([\mathrm{M}+\mathrm{H}]^{+}\right)$Calcd. for $\mathrm{C}_{32} \mathrm{H}_{42} \mathrm{~N}_{5} \mathrm{O}_{7}$ : 608.3084; Found: 608.3083 .

\section{Ac-Glu-Phe(4-iodo)-Ser-Thr-Val-NH (10c)}<smiles>CC(=O)N[C@@H](CCC(=O)O)C(=O)N[C@@H](Cc1ccc(I)cc1)C(=O)N[C@@H](CO)C(=O)N[C@H](C(=O)N[C@H](C(N)=O)C(C)C)[C@H](C)O</smiles>

General procedure $\mathrm{C}$ was followed using Rink amide resin (316 mg, $0.25 \mathrm{mmol})$. The crude product was purified by preparative HPLC to afford $10 \mathrm{c}$ as a white solid $(96.3 \mathrm{mg}, 51 \%) .{ }^{1} \mathrm{H}$ NMR (300 MHz, DMSO-d6) $\delta 12.06$ (s, 1H), 8.24 (d, J 7.6 Hz, 1H), 7.96 (d, J 7.8 Hz, 1H), 7.89 (d, J 8.3 Hz, 1H), 7.84 (d, J 8.1 Hz, 1H), 7.55 (dd, J 8.7, $2.9 \mathrm{~Hz}, 3 \mathrm{H}), 7.29$ (s, 1H), 7.11 (s, 1H), $7.08-7.00(\mathrm{~m}, 2 \mathrm{H}), 5.05(\mathrm{~d}, J 53.6 \mathrm{~Hz}, 2 \mathrm{H}), 4.56(\mathrm{td}, J 8.8,3.9 \mathrm{~Hz}, 1 \mathrm{H}), 4.42$ (q, J 6.5 $\mathrm{Hz}, 1 \mathrm{H}), 4.27$ (dd, J 8.1, 3.4 Hz, 1H), $4.22-4.04$ (m, 3H), 3.67-3.54 (m, 2H), 3.01 (dd, J 13.9, $4.0 \mathrm{~Hz}, 1 \mathrm{H}), 2.73$ (dd, J 13.8, 9.5 Hz, 1H), 2.15 (t, J 7.9 Hz, 2H), 2.04-1.93 (h, J 6.5 Hz, 1H), $1.86-1.54(\mathrm{~m}, 5 \mathrm{H}), 1.06(\mathrm{~d}, J 6.3 \mathrm{~Hz}, 3 \mathrm{H}), 0.85$ (d, J 6.8 Hz, 3H), $0.82(\mathrm{~d}, J 6.8 \mathrm{~Hz}, 3 \mathrm{H}) .{ }^{13} \mathrm{C}$ NMR (75 MHz, DMSO- $d 6) \delta 173.9,172.9,171.1,170.7,170.4,169.7,169.4,137.5,136.6$, 131.8, 92.2, 66.3, 61.7, 58.2, 57.4, 54.9, 53.2, 51.9, 37.0, 30.3, 30.1, 27.2, 22.5, 19.7, 19.3, 17.7 . HRMS (ESI) m/z: $\left([\mathrm{M}+\mathrm{H}]^{+}\right)$Calcd. for $\mathrm{C}_{28} \mathrm{H}_{42} \mathrm{IN}_{6} \mathrm{O}_{10}$ : 749.2007; Found: 749.2002. 
<smiles>CCCCOC(=O)Cc1ccc(CC(NC(=O)[C@H](CCC(=O)O)NC(=O)O)C(=O)N[C@@H](CO)C(=O)N[C@H](C(=O)N[C@H](C(N)=O)C(C)C)[C@H](C)O)cc1</smiles>

General procedure D was followed using $10 \mathrm{c}(74.8 \mathrm{mg}, 0.1 \mathrm{mmol})$ and tert-butyl chloroacetate $(42.9 \mu \mathrm{L}, 0.3 \mathrm{mmol})$ as the starting material. The crude product was subjected to the Strong Cation Exchange column (SCX) to remove residual HE and then the product was eluted with $\mathrm{MeCN}$. The crude product was then purified by preparative HPLC to afford 11c as a white solid (30.7 mg, 42\%). ${ }^{1} \mathrm{H}$ NMR (300 MHz, DMSO- $\left.d 6\right) \delta 12.06(\mathrm{~s}, 1 \mathrm{H}), 8.20(\mathrm{~d}, J 7.6 \mathrm{~Hz}, 1 \mathrm{H})$, $7.96(\mathrm{~d}, J 7.7 \mathrm{~Hz}, 1 \mathrm{H}), 7.89$ (d, J $8.1 \mathrm{~Hz}, 1 \mathrm{H}), 7.82$ (d, J 8.1 Hz, 1H), 7.55 (d, J 8.9 Hz, 1H), 7.29 (s, 1H), 7.17 (d, J 7.9 Hz, 2H), 7.09 (d, J 7.9 Hz, 3H), 4.83 (s, 2H), 4.54 (d, J $11.1 \mathrm{~Hz}$, $1 \mathrm{H}), 4.42$ (q, $J 6.5 \mathrm{~Hz}, 1 \mathrm{H}), 4.18-4.06(\mathrm{~m}, 3 \mathrm{H}), 3.66-3.58(\mathrm{~m}, 2 \mathrm{H}), 3.47(\mathrm{~s}, 2 \mathrm{H}), 3.05(\mathrm{~d}, J$ $12.6 \mathrm{~Hz}, 1 \mathrm{H}), 2.76(\mathrm{dd}, J 13.9,9.7 \mathrm{~Hz}, 1 \mathrm{H}), 2.13(\mathrm{t}, J 7.9 \mathrm{~Hz}, 2 \mathrm{H}), 2.05-1.92(\mathrm{~m}, 1 \mathrm{H}), 1.81$ $(\mathrm{s}, 3 \mathrm{H}), 1.76-1.58(\mathrm{~m}, 2 \mathrm{H}), 1.39(\mathrm{~s}, 9 \mathrm{H}), 1.06(\mathrm{~d}, J 6.3 \mathrm{~Hz}, 3 \mathrm{H}), 0.84$ (apparent dd, J 9.0, 6.7 $\mathrm{Hz}, 6 \mathrm{H}) .{ }^{13} \mathrm{C}$ NMR $\left(75 \mathrm{MHz}, \mathrm{DMSO}-d_{6}\right) \delta 174.1,172.9,171.1,171.1,170.5,170.4,169.7$, 169.5, 136.1, 132.6, 129.2, 128.8, 80.1, 66.3, 61.7, 58.2, 57.5, 54.9, 53.5, 51.9, 41.3, 36.9, 30.3, 30.1, 27.7, 27.2, 22.5, 19.7, 19.3, 17.7. HRMS (ESI) m/z: $\left([\mathrm{M}+\mathrm{H}]^{+}\right)$Calcd. for $\mathrm{C}_{34} \mathrm{H}_{53} \mathrm{~N}_{6} \mathrm{O}_{12}$ : 737.3721; Found: 737.3716.

\section{Ac-Glu-Phe(4-iodo)-Tyr-Lys-Val-NH 2 (10d)}<smiles>CC(=O)N[C@@H](CCC(=O)O)C(=O)N[C@@H](Cc1ccc(I)cc1)C(=O)N[C@@H](Cc1ccc(O)cc1)C(=O)N[C@@H](CCCCN)C(=O)N[C@H](C(N)=O)C(C)C</smiles> 
General procedure $\mathrm{C}$ was followed using Rink amide resin $(316 \mathrm{mg}, 0.25 \mathrm{mmol})$. The crude product was purified by preparative HPLC to afford $10 d$ as a white solid $(98.3 \mathrm{mg}, 46 \%) .{ }^{1} \mathrm{H}$ NMR (300 MHz, DMSO-d6) $\delta 12.09$ (s, 1H), 9.16 (s, 1H), 8.14 (d, J 7.9 Hz, 1H), 8.06 (d, J 7.9 Hz, 1H), 7.98 (d, J 7.8 Hz, 1H), 7.85 (d, J 8.2 Hz, 1H), 7.68-765 (m, 3H), 7.54 (d, J 8.2 Hz, 2H), $7.41(\mathrm{~s}, 1 \mathrm{H}), 7.06-6.96(\mathrm{~m}, 5 \mathrm{H}), 6.63(\mathrm{~d}, J 8.5 \mathrm{~Hz}, 2 \mathrm{H}), 4.48-4.39(\mathrm{~m}, 2 \mathrm{H}), 4.36-4.27$ (m, 1H), 4.20-4.10 (m, 2H), 2.92 (dd, J 14.1, $4.2 \mathrm{~Hz}, 2 \mathrm{H}), 2.81-2.63(\mathrm{~m}, 4 \mathrm{H}), 2.15$ (t, J 7.8 Hz, 2H), 2.01-1.90 (m, 1H), $1.86-1.45(\mathrm{~m}, 9 \mathrm{H}), 1.38-1.23(\mathrm{~m}, 2 \mathrm{H}), 0.85(\mathrm{t}, J 6.7 \mathrm{~Hz}, 6 \mathrm{H}) .{ }^{13} \mathrm{C}$ NMR (75 MHz, DMSO- $d 6) \delta 173.9,172.8,171.2,171.1,170.9,170.5,169.5,155.8,137.4$, 136.7, 131.7, 130.1, 127.6, 114.9, 92.2, 57.3, 54.1, 53.4, 52.4, 51.8, 36.8, 36.5, 31.3, 30.6, 30.1, 26.9, 26.7, 22.5, 22.2, 19.3, 17.9. HRMS (ESI) m/z: $\left([\mathrm{M}+\mathrm{H}]^{+}\right)$Calcd. for $\mathrm{C}_{36} \mathrm{H}_{51} \mathrm{IN}_{7} \mathrm{O}_{9}$ : 852.2793; Found: 852.2764.

\section{Ac-Glu-pCMF(OtBu)-Tyr-Lys-Val-NH2 (11d)}<smiles>CCCCOC(=O)Cc1ccc(CC(NC(=O)[C@H](CCC(=O)O)NC(=O)OCc2ccccc2)C(=O)N[C@@H](Cc2ccc(O)cc2)C(=O)N[C@@H](CCCCN)C(=O)N[C@H](C(N)=O)C(C)C)cc1</smiles>

General procedure D was followed using $10 d(85.1 \mathrm{mg}, 0.1 \mathrm{mmol})$ and tert-butyl chloroacetate (42.9 $\mu \mathrm{L}, 0.3 \mathrm{mmol})$ as the starting material. The crude product was subjected to the Strong Cation Exchange column (SCX) to remove residual HE and then the product was eluted with $\mathrm{MeCN}$. The crude product was then purified by preparative HPLC to afford 11d as a white solid (32.9 mg, 39\%). ${ }^{1} \mathrm{H}$ NMR (300 MHz, MeOD-d $) \delta 7.16$ (d, J 8.1 Hz, 2H), $7.14-7.06$ (m, $4 \mathrm{H}), 6.78-6.69(\mathrm{~m}, 2 \mathrm{H}), 4.50$ (ddd, J 9.1, 5.4, $1.3 \mathrm{~Hz}, 2 \mathrm{H}), 4.41$ (dd, J 8.9, $5.4 \mathrm{~Hz}, 1 \mathrm{H}), 4.29$ - $4.16(\mathrm{~m}, 2 \mathrm{H}), 3.51$ (s, 2H), 3.08 (dt, J 14.1, $5.6 \mathrm{~Hz}, 2 \mathrm{H}), 3.03-2.80$ (m, 5H), 2.30 (t, J 7.4 $\mathrm{Hz}, 2 \mathrm{H}), 2.10$ (h, J 6.7 Hz, 1H), 1.98 (s, 3H), $1.92-1.65$ (m, 5H), 1.45 (s, 11H), 1.00 (apparent dd, $J 6.8,3.7 \mathrm{~Hz}, 6 \mathrm{H})$. (Exchange protons cannot be observed in MeOD-d4.) ${ }^{13} \mathrm{C}$ NMR (75 MHz, MeOD- $\left.d_{4}\right) \delta 176.4,176.1,174.1,173.9,173.7,173.6,173.6,173.0,157.4,136.7,134.7$, $131.3,130.5,130.4,128.9,116.4,82.1,59.9,56.8,56.4,54.7,54.3,42.9,40.6,37.7,37.3,32.2$, 
31.9, 30.9, 28.9, 27.9, 27.5, 23.5, 22.6, 19.8, 18.6. HRMS (ESI) m/z: ([M+H] $\left.]^{+}\right)$Calcd. for $\mathrm{C}_{42} \mathrm{H}_{62} \mathrm{~N}_{7} \mathrm{O}_{11}$ : 840.4507; Found: 840.4510 .

\section{Ac-Glu-Phe(4-bromo)-Ser-Thr-Val-NH 2 (10e)}<smiles>CC(=O)N[C@@H](CCC(=O)O)C(=O)N[C@@H](Cc1ccc(Br)cc1)C(=O)N[C@@H](CO)C(=O)N[C@H](C(=O)N[C@H](C(N)=O)C(C)C)[C@H](C)O</smiles>

General procedure $\mathrm{C}$ was followed using Rink amide resin (316 $\mathrm{mg}, 0.25 \mathrm{mmol}$ ). The crude product was purified by preparative HPLC to afford 10e as a white solid (112.3 $\mathrm{mg}, 64 \%) .{ }^{1} \mathrm{H}$ NMR (300 MHz, DMSO-d6) $\delta 12.06$ (s, 1H), 8.25 (d, J 7.6 Hz, 1H), 7.96 (d, J 7.8 Hz, 1H), $7.91(\mathrm{~d}, J 8.2 \mathrm{~Hz}, 1 \mathrm{H}), 7.84(\mathrm{~d}, J 8.1 \mathrm{~Hz}, 1 \mathrm{H}), 7.55(\mathrm{~d}, J 8.8 \mathrm{~Hz}, 1 \mathrm{H}), 7.39$ (d, $J 8.3 \mathrm{~Hz}, 2 \mathrm{H})$, $7.30(\mathrm{~s}, 1 \mathrm{H}), 7.18(\mathrm{~d}, J 8.5 \mathrm{~Hz}, 2 \mathrm{H}), 7.11(\mathrm{~s}, 1 \mathrm{H}), 5.07(\mathrm{~d}, J 57.6 \mathrm{~Hz}, 2 \mathrm{H}), 4.61-4.53(\mathrm{~m}, 1 \mathrm{H})$, 4.43 (q, $J 6.3 \mathrm{~Hz}, 1 \mathrm{H}), 4.27$ (dd, $J 8.1,3.4 \mathrm{~Hz}, 1 \mathrm{H}), 4.22-4.03(\mathrm{~m}, 3 \mathrm{H}), 3.67-3.54(\mathrm{~m}, 2 \mathrm{H})$, $3.03(\mathrm{dd}, J$ 13.9, $4.0 \mathrm{~Hz}, 1 \mathrm{H}), 2.75$ (dd, $J 13.9,9.5 \mathrm{~Hz}, 1 \mathrm{H}), 2.15(\mathrm{t}, J 7.9 \mathrm{~Hz}, 2 \mathrm{H}), 2.04-1.93$ $(\mathrm{m}, 1 \mathrm{H}), 1.85-1.57(\mathrm{~m}, 5 \mathrm{H}), 1.06$ (d, J $6.3 \mathrm{~Hz}, 3 \mathrm{H}), 0.85$ (d, J $6.8 \mathrm{~Hz}, 3 \mathrm{H}), 0.82$ (d, J $6.8 \mathrm{~Hz}$, $3 \mathrm{H}) .{ }^{13} \mathrm{C}$ NMR (75 MHz, DMSO-d6) $\delta 173.9,172.9$, 171.1, 170.8, 170.4, 169.7, 169.5, 137.1, 131.6, 130.8, 119.5, 66.3, 61.7, 58.2, 57.5, 54.9, 53.2, 51.9, 36.9, 30.3, 30.1, 27.2, 22.5, 19.7, 19.3, 17.7. HRMS (ESI) m/z: $\left([\mathrm{M}+\mathrm{H}]^{+}\right)$Calcd. for $\mathrm{C}_{28} \mathrm{H}_{42}{ }^{79} \mathrm{BrN}_{6} \mathrm{O}_{10}$ : 701.2146; Found: 701.2142 .

Ac-Glu- Pmp(OEt) $)_{2}-$ Ser-Thr-Val-NH 2 (11e)<smiles>CCO[PH](Cc1ccc(CC(NC(=O)[C@H](CCC(=O)O)NC(C)=O)C(=O)N[C@@H](CO)C(=O)N[C@H](C(=O)N[C@H](C(N)=O)C(C)C)[C@H](C)O)cc1)(OCC)OCC</smiles> 
General procedure D was followed except that extra DIPEA $(105.1 \mu \mathrm{L}, 0.6 \mathrm{mmol})$ was added. 10e $(70 \mathrm{mg}, 0.1 \mathrm{mmol})$ and diethyl chloromethylphosphonate $(46.6 \mu \mathrm{L}, 0.3 \mathrm{mmol})$ were used as the starting material. The crude product was subjected to the Strong Cation Exchange column (SCX) to remove residual HE and then the product was eluted with $\mathrm{MeCN}$. The crude product was then purified by preparative HPLC to afford 11e as a white solid (23.6 mg, 31\%) ${ }^{1} \mathrm{H}$ NMR (300 MHz, DMSO-d6) $\delta 12.04$ (s, 1H), 8.19 (d, J 7.6 Hz, 1H), 7.96 (d, J 7.8 Hz, 1H), $7.90(\mathrm{~d}, J 8.2 \mathrm{~Hz}, 1 \mathrm{H}), 7.82(\mathrm{~d}, J 8.1 \mathrm{~Hz}, 1 \mathrm{H}), 7.55(\mathrm{~d}, J 8.9 \mathrm{~Hz}, 1 \mathrm{H}), 7.29$ (d, J $2.2 \mathrm{~Hz}, 1 \mathrm{H})$, $7.21-7.08$ (m, 5H), $5.05(\mathrm{~d}, J 54.6 \mathrm{~Hz}, 2 \mathrm{H}), 4.55(\mathrm{td}, J 8.8,8.1,3.8 \mathrm{~Hz}, 1 \mathrm{H}), 4.42(\mathrm{q}, J 6.4 \mathrm{~Hz}$, 1H), 4.26 (dd, J 8.1, $3.4 \mathrm{~Hz}, 1 \mathrm{H}), 4.20-4.05$ (m, 3H), 3.91 (dq, J 8.3, 7.1 Hz, 4H), $3.68-3.55$ (m, 2H), 3.17 (s, 1H), 3.06 (s, 2H), $2.75(\mathrm{dd}, J 13.9,9.8 \mathrm{~Hz}, 1 \mathrm{H}), 2.13$ (t, J 7.9 Hz, 2H), 1.97 (dp, $J 13.7,6.9 \mathrm{~Hz}, 1 \mathrm{H}), 1.85-1.57$ (m, 5H), 1.15 (t, J 7.0 Hz, 6H), 1.06 (d, J 6.3 Hz, 3H), 0.84 (apparent dd, $J$ 9.1, $6.8 \mathrm{~Hz}, 6 \mathrm{H}) .{ }^{13} \mathrm{C}$ NMR (75 MHz, DMSO-d6) $\delta 174.1,172.9,171.1$, 171.1, 170.4, 169.7, 169.5, 136.0 (d, J 3.5 Hz), 130.0 (d, J 8.9 Hz), 129.4 (d, $J 6.3 \mathrm{~Hz}), 129.2$ (d, J 2.7 Hz), 66.3, 61.7, 61.4, 61.3, 58.2, 57.5, 54.9, 53.5, 52.1, 36.9, 31.9 (d, $\left.J_{\mathrm{C}-\mathrm{P}} 134.9 \mathrm{~Hz}\right)$, 30.3, 30.1, 27.3, 22.5, 19.7, 19.3, 17.7, 16.3, 16.2. ${ }^{31} \mathrm{P}$ NMR (121 MHz, DMSO) $\delta ~ 26.62$. HRMS (ESI) m/z: $\left([\mathrm{M}+\mathrm{H}]^{+}\right)$Calcd. for $\mathrm{C}_{33} \mathrm{H}_{54} \mathrm{~N}_{6} \mathrm{O}_{13} \mathrm{P}:$ 773.3486; Found: 773.3475.

\section{Ac-Pro-Gln-Asp-Lys-Glu-Phe(4-iodo)-Tyr-Lys-Val-Lys-NH2 (10f)}

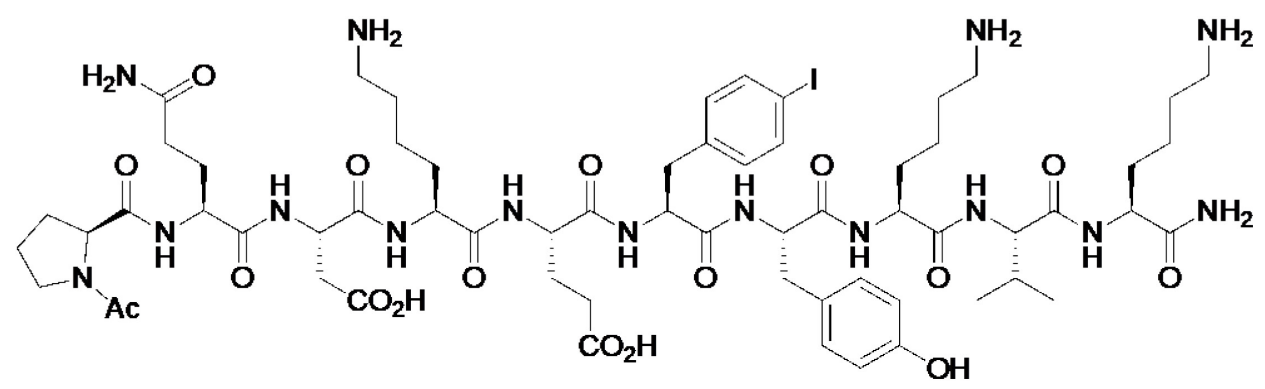

General procedure $\mathrm{C}$ was followed using Rink amide resin $(316 \mathrm{mg}, 0.25 \mathrm{mmol})$. The crude product was purified by preparative HPLC to afford 10 as a white solid (158.7 mg, 44\%). RP$\operatorname{HPLC}(214 \mathrm{~nm}): 98 \%\left(\mathrm{t}_{\mathrm{R}}=0.996 \mathrm{~min}\right)$. HRMS (ESI) m/z: $\left([\mathrm{M}+\mathrm{H}]^{+}\right)$Calcd. for $\mathrm{C}_{62} \mathrm{H}_{95} \mathrm{IN}_{15} \mathrm{O}_{17}$ : 1448.6075; Found: 1448.6114. 


\section{Ac-Pro-GIn-Asp-Lys-Glu- $p$ CMF(OtBu)-Tyr-Lys-Val-Lys-NH 2 (11f)}<smiles>CCCCC(=O)OCc1ccc(CC(NC(=O)C(CCCCN)NC(=O)C(CCCCN)NC(=O)C(CCCCN)NC(=O)C(CCC(N)=O)NC(=O)C2CCCN2C(C)(C)C)C(=O)NC(Cc2ccc(O)cc2)C(=O)NC(CCC(=O)O)C(=O)NC(C(=O)NC(CCCCN)C(N)=O)C(C)C)cc1</smiles>

General procedure D was followed using $10 \mathbf{f}(144.7 \mathrm{mg}, 0.1 \mathrm{mmol})$ and tert-butyl chloroacetate $(42.9 \mu \mathrm{L}, 0.3 \mathrm{mmol})$ as the starting material. The crude product was subjected to the Strong Cation Exchange column (SCX) to remove residual HE and then the product was eluted with $\mathrm{MeCN}$. The crude product was purified by preparative HPLC to afford $\mathbf{1 1 f}$ as a white solid (40.2 mg, 28\%). RP-HPLC (214 nm): 98\% ( $\left.\mathrm{t}_{\mathrm{R}}=1.079 \mathrm{~min}\right)$. HRMS (ESI) m/z: $\left([\mathrm{M}+\mathrm{H}]^{+}\right) \mathrm{Calcd}$. for $\mathrm{C}_{68} \mathrm{H}_{106} \mathrm{~N}_{15} \mathrm{O}_{19}$ : 1436.7789; Found: 1436.7740 .

\section{Ac-Glu-Tyr( $\left.\mathrm{PO}_{3} \mathrm{H}_{2}\right)$-Tyr-Lys-Val-NH2 (12a)}<smiles>CC(C)[C@H](NC(=O)[C@@H](NC(=O)[C@H](CO)NC(=O)[C@H](Cc1ccc(O[PH](O)(O)O)cc1)NC(=O)[C@H](CCC(=O)O)NC(=O)O)[C@@H](C)O)C(N)=O</smiles>

General procedure C was followed using Rink amide resin (316 mg, $0.25 \mathrm{mmol}$ ). The crude product was purified by preparative HPLC to afford $12 \mathrm{a}$ as a white solid $(95.1 \mathrm{mg}, 53 \%) .{ }^{1} \mathrm{H}$ NMR (300 MHz, $\left.\mathrm{CDCl}_{3}\right) \delta 8.22(\mathrm{~d}, J 7.6 \mathrm{~Hz}, 1 \mathrm{H}), 7.97(\mathrm{~d}, J 7.8 \mathrm{~Hz}, 1 \mathrm{H}), 7.86(\mathrm{dd}, J$ 18.0, 8.1 Hz, 2H), $7.54(\mathrm{~d}, J 8.9 \mathrm{~Hz}, 1 \mathrm{H}), 7.28(\mathrm{~s}, 1 \mathrm{H}), 7.18(\mathrm{~d}, J 8.6 \mathrm{~Hz}, 2 \mathrm{H}), 7.10(\mathrm{~s}, 1 \mathrm{H}), 7.01(\mathrm{~d}, J 8.5$ Hz, 2H), $5.14(\mathrm{~s}, 2 \mathrm{H}), 4.57-4.50(\mathrm{~m}, 1 \mathrm{H}), 4.43(\mathrm{~d}, J 6.9 \mathrm{~Hz}, 1 \mathrm{H}), 4.27(\mathrm{dd}, J 8.1,3.4 \mathrm{~Hz}, 1 \mathrm{H})$, $4.16-4.10$ (m, 3H), $3.64-3.60$ (m, 2H), 3.03 (dd, $J$ 14.0, 3.9 Hz, 1H), 2.75 (dd, $J$ 14.0, 9.5 Hz, 1H), 2.17 (t, J 7.8 Hz, 2H), 1.99 (h, J 6.7 Hz, 1H), 1.82 (s, 4H), 1.65 (ddd, J 14.2, 8.5, 6.4 $\mathrm{Hz}, 1 \mathrm{H}), 1.06(\mathrm{~d}, J 6.3 \mathrm{~Hz}, 3 \mathrm{H}), 0.84(\mathrm{dd}, J 9.1,6.8 \mathrm{~Hz}, 6 \mathrm{H}) .{ }^{13} \mathrm{C} \mathrm{NMR}\left(75 \mathrm{MHz}, \mathrm{CDCl}_{3}\right) \delta$ 173.9 , 172.9, 171.1, 171.0, 170.4, 169.7, 169.5, 133.1, 130.2, 119.6, 119.5, 66.3, 61.7, 58.2, 
57.4, 54.9, 53.5, 51.9, 36.6, 30.2, 30.1, 27.2, 22.4, 19.6, 19.3, 17.7. ${ }^{31} \mathrm{P}$ NMR (121 MHz, DMSO) $\delta$-6.19. HRMS (ESI) m/z: $\left([\mathrm{M}+\mathrm{H}]^{+}\right)$Calcd. for $\mathrm{C}_{28} \mathrm{H}_{44} \mathrm{~N}_{6} \mathrm{O}_{14} \mathrm{P}: 719.2653$; Found: 719.2647.

\section{Ac-Glu- $p$ CMF-Ser-Thr-Val-NH2 (12b)}<smiles>[CH]C(=O)N[C@@H](CCC(=O)O)C(=O)N[C@@H](Cc1ccc(CC(=O)O)cc1)C(=O)N[C@@H](CO)C(=O)N[C@H](C(=O)N[C@H](C(N)=O)C(C)C)[C@H](C)O</smiles>

Compound 11c (20 mg, $0.027 \mathrm{mmol})$ was treated with neat TFA $(1 \mathrm{~mL})$. The reaction solution was stirred at room temperature until the starting material was fully consumed (monitored by LCMS). The TFA was evaporated under a stream of $\mathrm{N}_{2}$ and the crude material was purified by preparative HPLC to afford $\mathbf{1 2 b}$ as a white solid (7.2 mg, 40\%). ${ }^{1} \mathrm{H}$ NMR (300 MHz, DMSOd6) $\delta 12.13(\mathrm{~s}, 1 \mathrm{H}), 8.21(\mathrm{~d}, J 7.6 \mathrm{~Hz}, 1 \mathrm{H}), 7.97(\mathrm{~d}, J 7.8 \mathrm{~Hz}, 1 \mathrm{H}), 7.89(\mathrm{~d}, J 8.1 \mathrm{~Hz}, 1 \mathrm{H}), 7.83$ $(\mathrm{d}, J 8.1 \mathrm{~Hz}, 1 \mathrm{H}), 7.55(\mathrm{~d}, J 8.9 \mathrm{~Hz}, 1 \mathrm{H}), 7.29(\mathrm{~s}, 1 \mathrm{H}), 7.20-7.06(\mathrm{~m}, 5 \mathrm{H}), 4.55(\mathrm{td}, J 9.2,3.8$ $\mathrm{Hz}, 1 \mathrm{H}), 4.43$ (q, J6.4 Hz, 1H), 4.27 (dd, J8.1, 3.4 Hz, 1H), 4.13 (tdd, J 13.0, 8.9, 6.0 Hz, 3H), 3.62 (dt, $J 12.2,5.6 \mathrm{~Hz}, 2 \mathrm{H}), 3.49$ (s, 2H), 3.05 (dd, $J 14.1,3.9 \mathrm{~Hz}, 1 \mathrm{H}), 2.76$ (dd, $J$ 14.0, 9.7 Hz, 1H), 2.13 (t, J 7.9 Hz, 2H), 1.99 (h, J 6.7 Hz, 1H), $1.86-1.54$ (m, 5H), 1.06 (d, J $6.3 \mathrm{~Hz}$, $3 \mathrm{H}), 0.84$ (apparent dd, $J$ 9.1, $6.8 \mathrm{~Hz}, 6 \mathrm{H}) .{ }^{13} \mathrm{C}$ NMR (75 MHz, DMSO-d6) $\delta$ 174.1, 172.9, $172.7,171.2$, 171.1 170.4, 169.7, 169.5, 136.0, 132.8, 129.2 , 129.1, 66.3, 61.7, 58.2, 57.5, 54.9, 53.5, 51.9, 36.9, 30.3, 30.1, 27.2, 22.5, 19.7, 19.3, 17.7. HRMS (ESI) m/z: ([M+H] $\left.]^{+}\right)$Calcd. for $\mathrm{C}_{30} \mathrm{H}_{45} \mathrm{~N}_{6} \mathrm{O}_{12}$ : 681.3095; Found: 681.3092. 
<smiles>CC(C)[C@H](NC(=O)[C@@H](NC(=O)[C@H](CO)NC(=O)[C@H](Cc1ccc(C[PH](O)(O)O)cc1)NC(=O)[C@H](CCC(=O)O)NC(=O)O)[C@@H](C)O)C(N)=O</smiles>

Compound 11e (18 mg, $0.023 \mathrm{mmol})$ was dissolved in $\mathrm{MeCN}(300 \mu \mathrm{L})$ and then treated with TMSI $(100 \mu \mathrm{L}, 0.7 \mathrm{mmol})$. The reaction solution was stirred for $2 \mathrm{~h}$ at room temperature, then $\mathrm{H}_{2} \mathrm{O}(300 \mu \mathrm{L})$ and DIPEA $(300 \mu \mathrm{L}, 0.86 \mathrm{mmol})$ were added and the mixture stirred for a further $30 \mathrm{~min}$. The reaction mixture was purified by SAX (MeCN, then $20 \% \mathrm{TFA}$ in $\mathrm{MeCN})$ and the product further purified by preparative HPLC to afford $12 \mathrm{c}$ as a white solid $(4.3 \mathrm{mg}, 26 \%) .{ }^{1} \mathrm{H}$ NMR (300 MHz, $\left.\mathrm{CDCl}_{3}\right) \delta 8.21(\mathrm{~d}, J 7.5 \mathrm{~Hz}, 1 \mathrm{H}), 7.98(\mathrm{~d}, J 7.7 \mathrm{~Hz}, 1 \mathrm{H}), 7.91$ (d, J 8.1 Hz, 1H), $7.82(\mathrm{~d}, J 8.0 \mathrm{~Hz}, 1 \mathrm{H}), 7.55$ (d, J $8.8 \mathrm{~Hz}, 1 \mathrm{H}), 7.29(\mathrm{~s}, 1 \mathrm{H}), 7.21-7.00(\mathrm{~m}, 5 \mathrm{H}), 5.13$ (s, $2 \mathrm{H}), 4.61-4.50(\mathrm{~m}, 1 \mathrm{H}), 4.42(\mathrm{q}, J 6.5 \mathrm{~Hz}, 1 \mathrm{H}), 4.26(\mathrm{dd}, J 8.1,3.4 \mathrm{~Hz}, 1 \mathrm{H}), 4.17-4.05$ (m, $3 \mathrm{H}), 3.63-3.58(\mathrm{~m}, 2 \mathrm{H}), 3.04(\mathrm{~d}, J 13.8 \mathrm{~Hz}, 1 \mathrm{H}), 2.88$ (d, $J 21.3 \mathrm{~Hz}, 2 \mathrm{H}), 2.73$ (dd, $J$ 13.9, $10.1 \mathrm{~Hz}, 1 \mathrm{H}), 2.12$ (t, J 7.9 Hz, 2H), 1.97 (p, J 6.9 Hz, 1H), 1.82 (s, 4H), 1.63 (dd, J 13.9, 7.4 $\mathrm{Hz}, 1 \mathrm{H}), 1.06$ (d, $J 6.2 \mathrm{~Hz}, 3 \mathrm{H}), 0.84(\mathrm{dd}, J 9.1,6.7 \mathrm{~Hz}, 6 \mathrm{H}) .{ }^{13} \mathrm{C}$ NMR (75 MHz, DMSO-d6) $\delta$ 173.9, 172.9, 171.1, 171.1, 170.4, 169.7, 169.6, 135.3 (d, J 3.2 Hz), 132.0 (d, J 9.0 Hz), 129.4 (d, J 6.4 Hz), 129.0 (d, J 2.7 Hz), 66.3, 61.6, 58.2, 57.5, 54.9, 53.6, 52.0, 36.9, 36.0, 30.2, 30.0, 27.2, 22.4, 19.6, 19.3, 17.7. ${ }^{31} \mathrm{P}$ NMR (121 MHz, DMSO-d6) $\delta 21.01$. HRMS (ESI) m/z: $\left([\mathrm{M}+\mathrm{H}]^{+}\right)$Calcd. for $\mathrm{C}_{29} \mathrm{H}_{46} \mathrm{~N}_{6} \mathrm{O}_{13} \mathrm{P}: 717.2860$; Found: 717.2857.

\section{(R)-Methyl 3-(4-iodophenyl)-2-(2,2,2-trifluoroacetamido)propanoate (R1)}<smiles>COC(=O)[C@H](Cc1ccc(I)cc1)NC(C)C</smiles>

General procedure A was followed using D-4-iodo-phenylalanine methyl ester ( $3 \mathrm{~g}, 10 \mathrm{mmol})$. The crude product was purified by flash chromatography gradient eluting from $0-25 \%$ EtOAc/n-heptane to afford $\boldsymbol{R} 1$ as a white solid $(3.0 \mathrm{~g}, 74 \%) .{ }^{1} \mathrm{H} \mathrm{NMR}\left(300 \mathrm{MHz}, \mathrm{CDCl}_{3}\right) \delta$ 
$7.64(\mathrm{~d}, J 8.3 \mathrm{~Hz}, 2 \mathrm{H}), 6.84-6.75(\mathrm{~m}, 3 \mathrm{H}), 4.85(\mathrm{dt}, J 7.6,5.5 \mathrm{~Hz}, 1 \mathrm{H}), 3.79(\mathrm{~s}, 3 \mathrm{H}), 3.26-$ $3.04(\mathrm{~m}, 2 \mathrm{H}) .{ }^{13} \mathrm{C} \mathrm{NMR}\left(75 \mathrm{MHz}, \mathrm{CDCl}_{3}\right) \delta 170.3,156.7$ (d, $\left.J_{\mathrm{C}-\mathrm{F}} 37.9 \mathrm{~Hz}\right), 138.1,134.4,131.2$, $115.6\left(\mathrm{~d}, J_{\mathrm{C}-\mathrm{F}} 287.6 \mathrm{~Hz}\right), 93.4,53.5,53.1,36.9 .{ }^{19} \mathrm{~F} \mathrm{NMR}(282 \mathrm{MHz}, \mathrm{CDCl} 3) \delta$-75.88. HRMS (ESI) $\mathrm{m} / \mathrm{z}:\left([\mathrm{M}+\mathrm{H}]^{+}\right)$Calcd. for $\mathrm{C}_{12} \mathrm{H}_{12} \mathrm{~F}_{3} \mathrm{INO}_{3}$ : 401.9814; Found: 401.9809.

(R)-Methyl 3-(4-(2-(tert-butoxy)-2-oxoethyl)phenyl)-2-(2,2,2trifluoroacetamido)propanoate $(R 3)$<smiles>CCCCOC(=O)Cc1ccc(C[C@H](NC(C)C)C(=O)OC)cc1</smiles>

General procedure B was followed using $\boldsymbol{R} \mathbf{1}(40.1 \mathrm{mg}, 0.1 \mathrm{mmol})$ and tert-butyl chloroacetate $(42.9 \mu \mathrm{L}, 0.3 \mathrm{mmol})$. The crude product was purified by flash chromatography gradient eluting from $0-25 \% \mathrm{EtOAc} / \mathrm{n}$-heptane to afford $\boldsymbol{R 3}$ as a colourless oil $(26.1 \mathrm{mg}, 67 \%)$. ${ }^{1} \mathrm{H}$ NMR (300

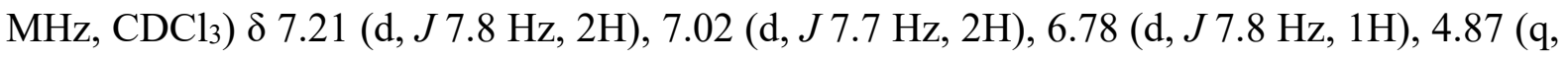
$J 6.1 \mathrm{~Hz}, 1 \mathrm{H}), 3.78$ (s, 3H), 3.49 (s, 2H), 3.17 (dq, $J 14.1,7.0,5.6 \mathrm{~Hz}, 2 \mathrm{H}), 1.42(\mathrm{~s}, 9 \mathrm{H}) .{ }^{13} \mathrm{C}$ NMR $\left(75 \mathrm{MHz}, \mathrm{CDCl}_{3}\right) \delta 170.9,170.5,156.7$ (d, $\left.J_{\mathrm{C}-\mathrm{F}} 37.7 \mathrm{~Hz}\right), 134.3,133.2,129.9,129.4$, $115.7\left(\mathrm{q}, J_{\mathrm{C}-\mathrm{F}} 287.7 \mathrm{~Hz}\right), 81.1,53.6,53.0,42.4,37.1,28.1 .{ }^{19} \mathrm{~F} \mathrm{NMR}\left(282 \mathrm{MHz}, \mathrm{CDCl}_{3}\right) \delta-$ 75.93. HRMS (ESI) m/z: ([M+H] $\left.]^{+}\right)$Calcd. for $\mathrm{C}_{18} \mathrm{H}_{23} \mathrm{~F}_{3} \mathrm{NO}_{5}$ : 390.1528; Found: 390.1522 .

\section{Biology Methods}


Protein expression and purification. Glycerol stocks of BL21(DE3) cells co-expressing GST-tagged human SOCS proteins with Elongin B (residues 1-118) and C (residues 17-112) were used. The human SOCS proteins information were as follows: CIS (residues 35-173, 202258), SOCS1 (residues 53-211), SOCS2 (residues 48-156) and SOCS3 (residues 22-225). The bacterial cells were inoculated into $10 \mathrm{~mL}$ of S-broth medium with antibiotics and incubated overnight at $37^{\circ} \mathrm{C}$. S-broth culture $(1 \mathrm{~L})$ with antibiotics was subsequently seeded and grown at $37{ }^{\circ} \mathrm{C}$ until an OD 600 of $0.7-0.8$ was reached. For CIS, SOCS1 and SOCS2, the culture was then cooled to $18{ }^{\circ} \mathrm{C}$, followed by $1 \mathrm{mM}$ IPTG induction overnight at $160 \mathrm{rpm}$. For SOCS3, the bacterial culture was induced by $1 \mathrm{mM} \mathrm{IPTG}$ for $2 \mathrm{~h}$ at $37^{\circ} \mathrm{C}$. The E. coli culture was then centrifuged at 5,000 g for $20 \mathrm{~min}$ at $4{ }^{\circ} \mathrm{C}$. The pellet was resuspended in $40 \mathrm{ml}$ protein lysis buffer (20mg lysozyme, $1 \mu \mathrm{g}$ DNAse I, 1 mM PMSF, 2 mM TCEP and 5 mM phenyl phosphate) and gently rotated at $4{ }^{\circ} \mathrm{C}$ for $30 \mathrm{~min}$ followed by $4 \mathrm{~min}$ sonication. The bacterial lysate was then centrifuged at 50,000 $\mathrm{g}$ for $20 \mathrm{~min}$ at $4{ }^{\circ} \mathrm{C}$. The supernatant was purified with a $1 \mathrm{~mL}$ GHS Sepharose in a $25 \mathrm{~mL}$ poly-prep column. The GHS-sepharose was then washed with $30 \mathrm{~mL}$ cleavage buffer (2 $\mathrm{mM}$ TCEP, $5 \mathrm{mM}$ phenyl phosphate in PBS) followed by overnight incubation with TEV enzyme at $4{ }^{\circ} \mathrm{C}$ to cleave GST tag. The cleaved protein was collected by gravity flow. The GST tag was the eluted from the resin with $5 \mathrm{mM}$ GHS. The protein and GST tag were further purified by size exclusion chromatography (SEC) using Superdex 200 16/60 column followed by condensation in Amicon Ultra centrifuge tubes respectively. The purified proteins were analysed by SDS-PAGE and Coomassie blue staining (InstantBlue $\left.{ }^{\circledR}\right)$.

Competition surface plasmon resonance assay. Competition SPR analysis of peptides was performed using Biacore 4000. Biotinylated JAK3 peptide (LLPLDKDpYYVVREPG) for CIS, SOCS1, SOCS2 and biotinylated GP130 (STASTVEpYSTVVHSG) for SOCS3 was immobilised to the Streptavidin coated chip (Series S SA, GH Healthcare). In the assay, 100 $\mathrm{nM}$ of human SOCS proteins were pre-incubated with test peptides in HBS-EP (10 mM HEPES, $150 \mathrm{mM} \mathrm{NaCl}, 3.4 \mathrm{mM}$ EDTA, 0.005\% Tween 20) running buffer in a 5-point dilution series (3-fold titration from 100 or $50 \mu \mathrm{M}$ ). Samples were allowed contact with the chip surface for $600 \mathrm{sec}$ followed by $120 \mathrm{sec}$ dissociation. The chip surface was then regenerated using regeneration buffer $(50 \mathrm{mM} \mathrm{NaOH}, 1 \mathrm{M} \mathrm{NaCl})$ for next running cycle. Raw data was generated and analysed by the Biacore evaluation software and Prism 8. The maximum SOCS protein binding from the running cycle without competition peptide was utilised to calculate the 
relative binding. The relative binding values to generate $\mathrm{IC}_{50}$ values was performed using GraphPad Prism version 8. 


\section{References}

(1) Guan, H.; Zhang, Q.; Walsh, P. J.; Mao, J., Nickel/photoredox-catalyzed asymmetric reductive cross-coupling of racemic alpha-chloro esters with aryl iodides. Angew. Chem. Int. Ed. Engl. 2020, 59, 5172-5177.

(2) Engle, S. M.; Kirkner, T. R.; Kelly, C. B., Org. Synth. 2019, 96, 455-473.

(3) Barluenga, J.; Alvarez-Gutiérrez, J. M.; Ballesteros, A.; González, J. M., Direct ortho iodination of beta- and gamma-aryl alkylamine derivatives. Angew. Chem. Int. Ed. Engl. 2007, $46,1281-1283$.

(4) Tulsi, N. S.; Downey, A. M.; Cairo, C. W., A protected 1bromophosphonomethylphenylalanine amino acid derivative (BrPmp) for synthesis of irreversible protein tyrosine phosphatase inhibitors. Bioorg. Med. Chem. 2010, 18, 8679-8686.

(5) Ge, J.; Wu, H.; Yao, S. Q., An unnatural amino acid that mimics phosphotyrosine. Chem. Commun. 2010, 46, 2980-2982.

(6) Chen, S.; Fahmi, N. E.; Wang, L.; Bhattacharya, C.; Benkovic, S. J.; Hecht, S. M., Detection of dihydrofolate reductase conformational change by FRET using two fluorescent amino acids. J. Am. Chem. Soc. 2013, 135, 12924-12927. 
Spectra of synthesized compounds

(S)-Methyl 3-(4-iodophenyl)-2-(2,2,2-trifluoroacetamido)propanoate (1)

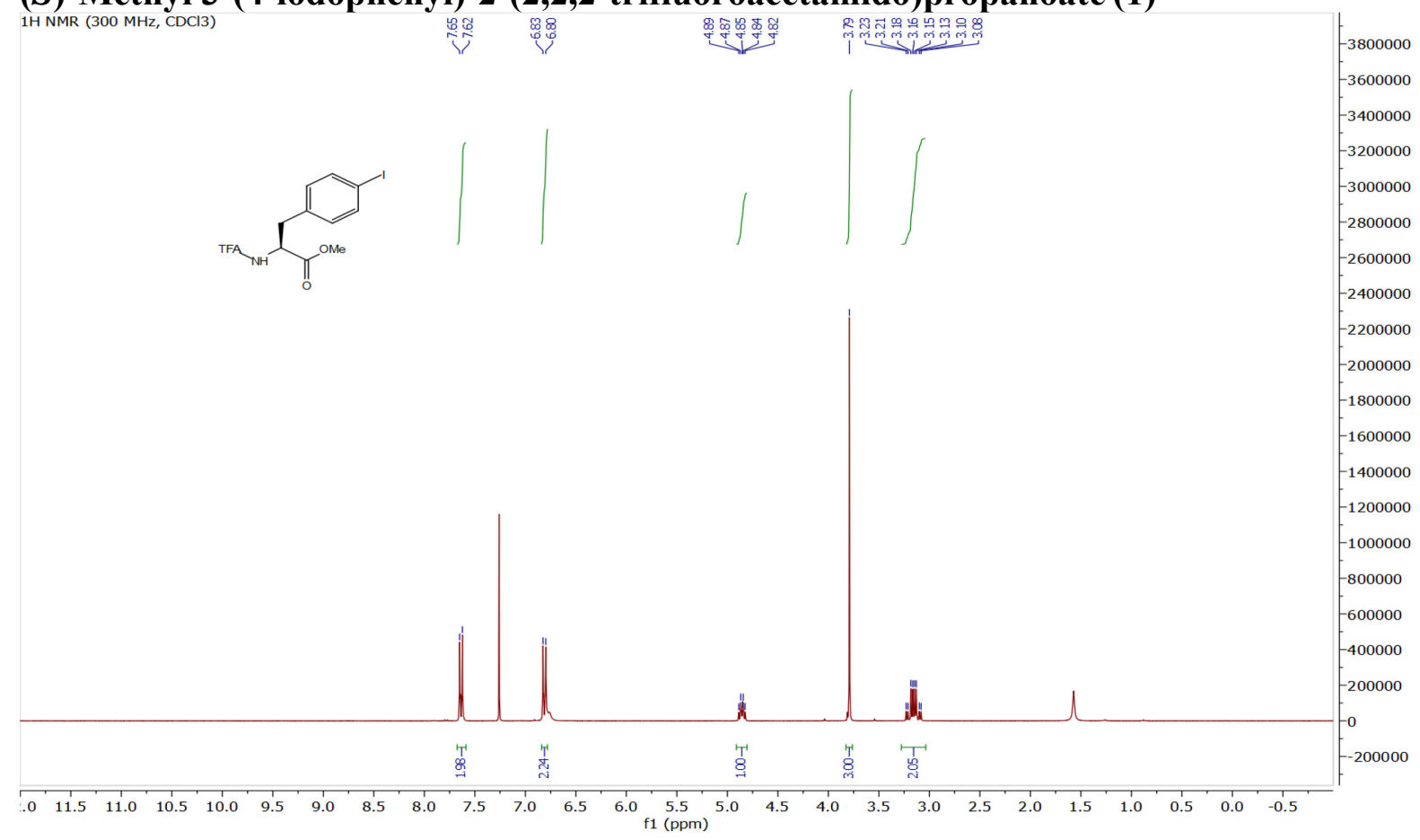

(S)-Methyl 2-(((benzyloxy)carbonyl)amino)-3-(4-iodophenyl)propanoate (4a)

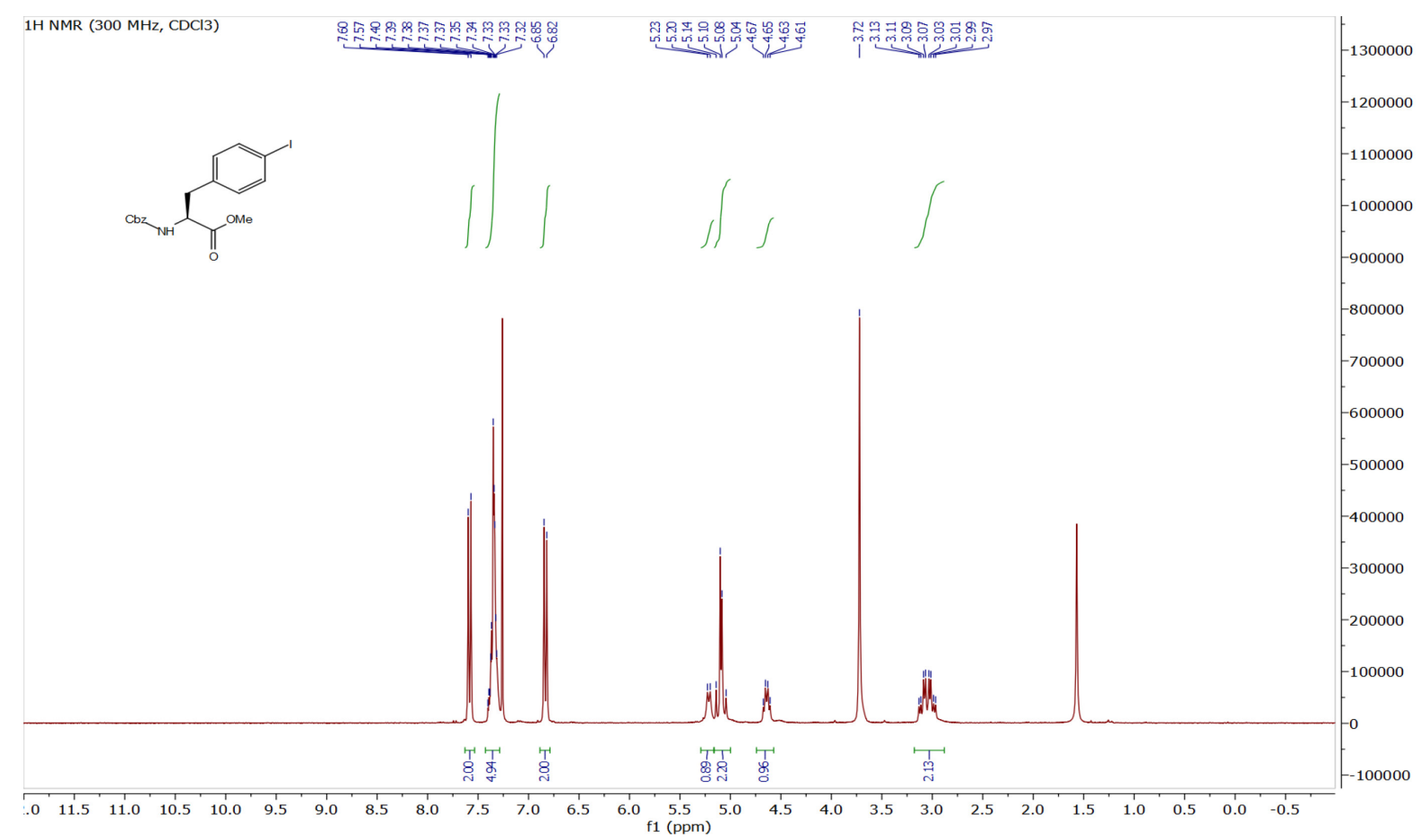


(S)-Methyl 2-((((9H-fluoren-9-yl)methoxy)carbonyl)amino)-3-(4-iodophenyl)propanoate (4c)

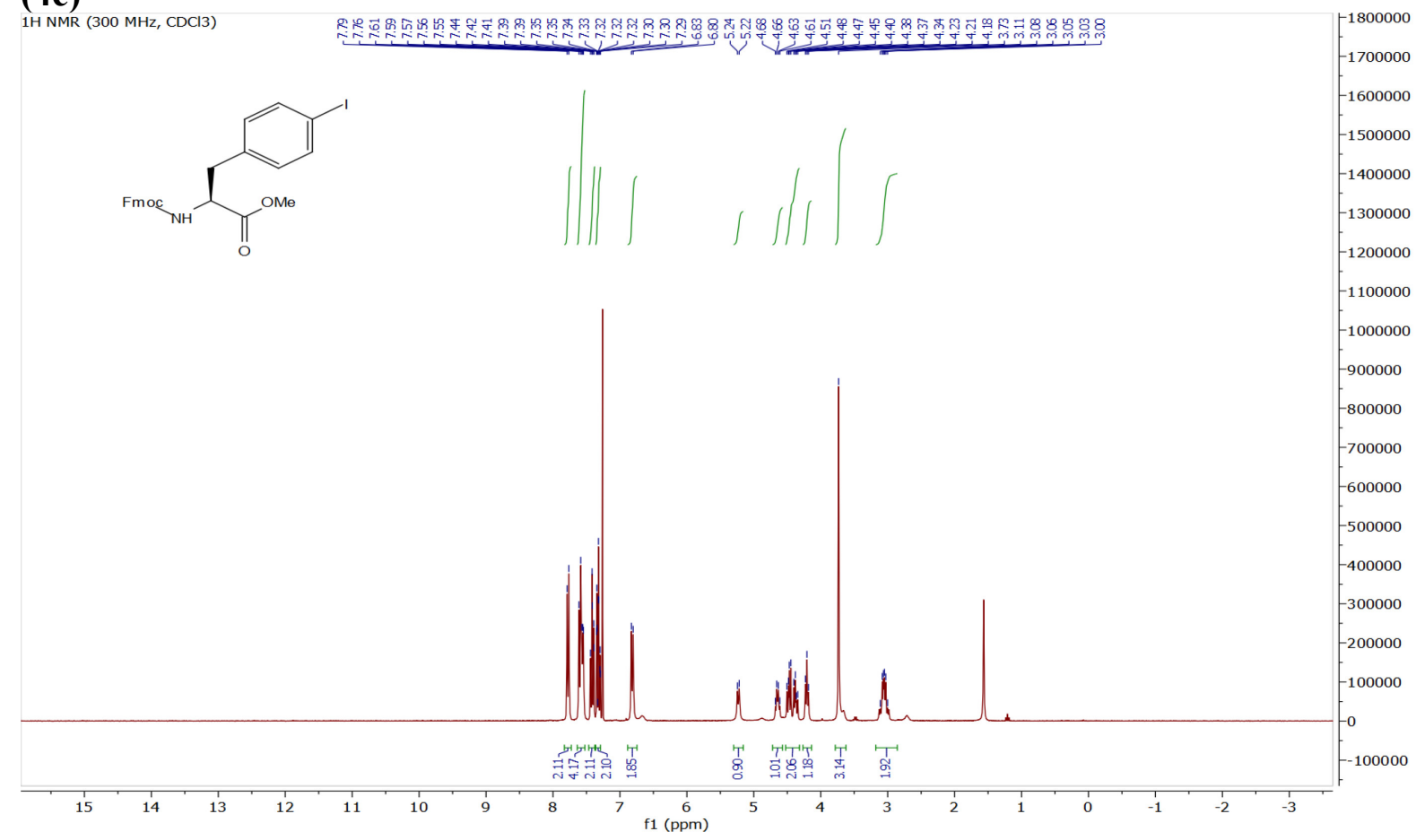

(S)-Methyl 2-((tert-butoxycarbonyl)amino)-3-(4-iodophenyl)propanoate (4d)

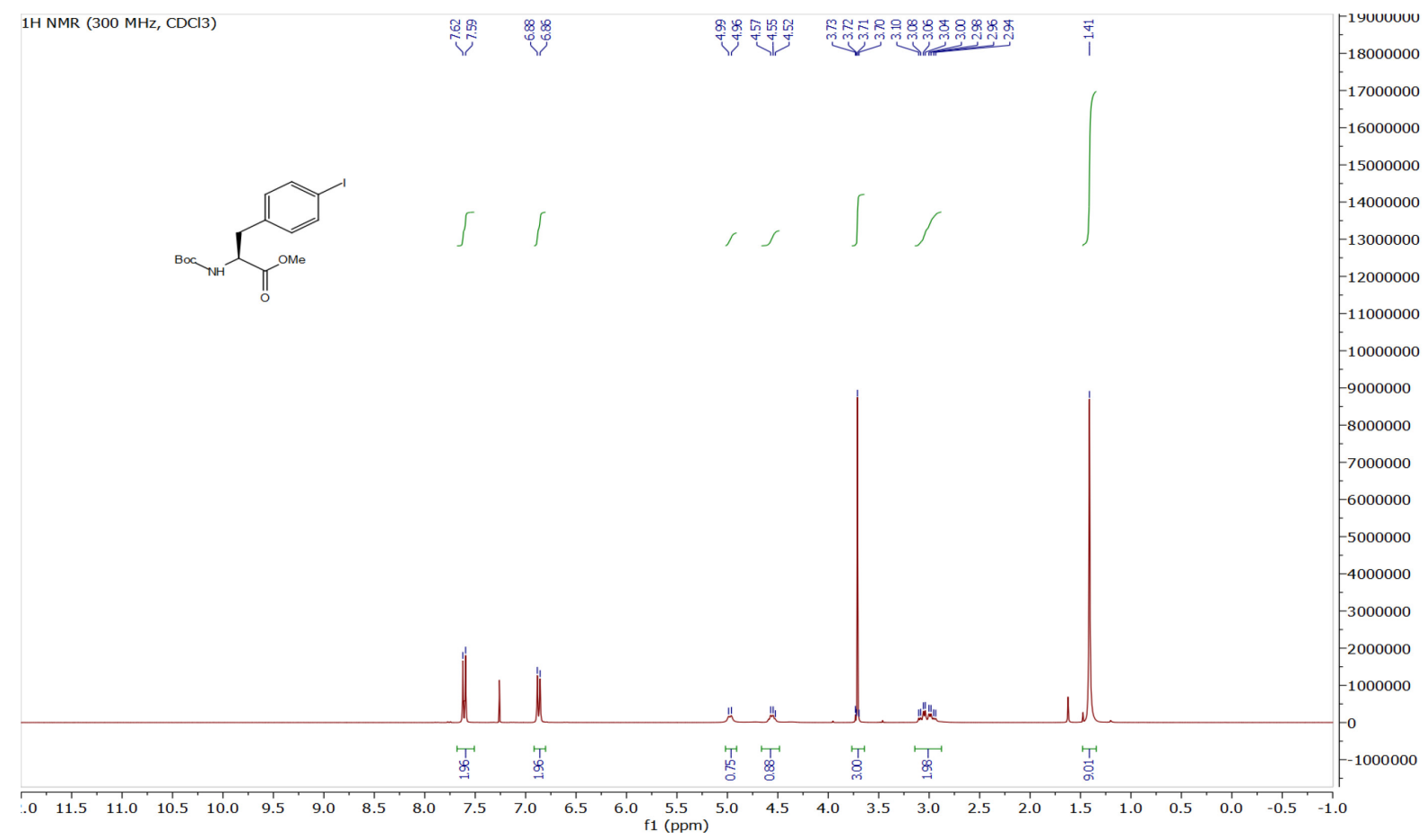


(S)-Methyl 2-(((benzyloxy)carbonyl)amino)-3-(4-bromophenyl)propanoate (6)

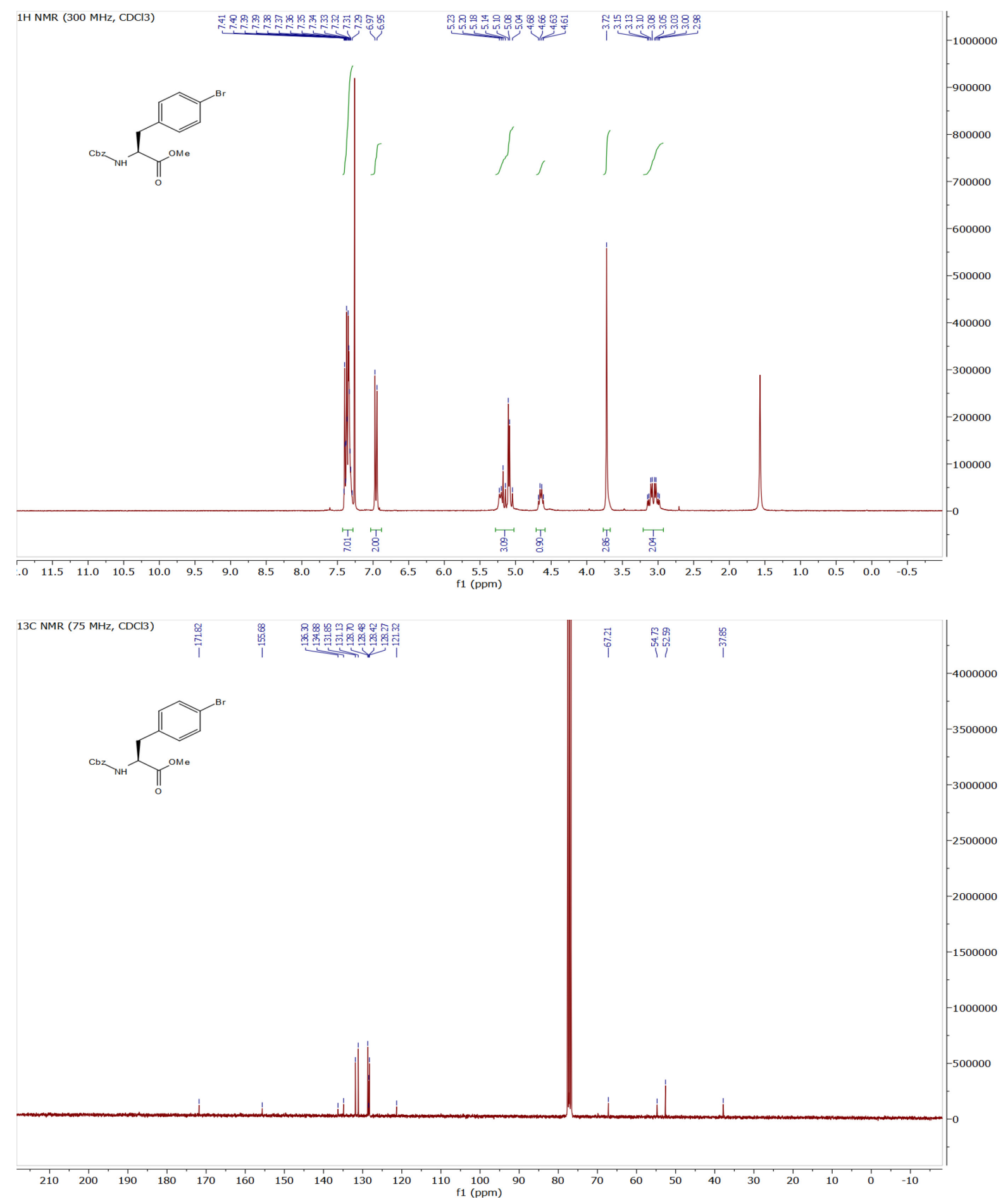


(S)-Methyl-3-(4-(2-(tert-butoxy)-2-oxoethyl)phenyl)-2-(2,2,2trifluoroacetamido)propanoate (3)
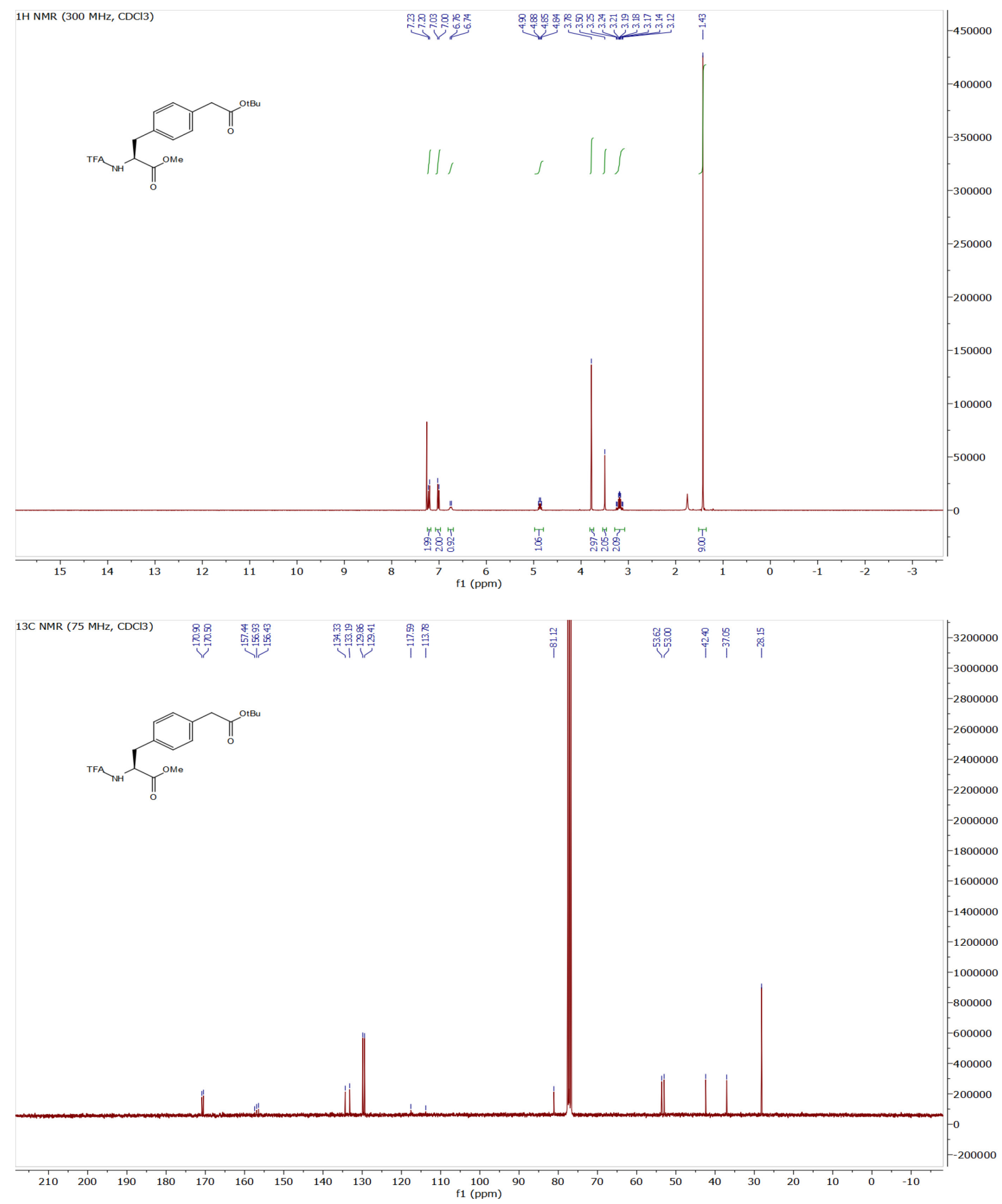


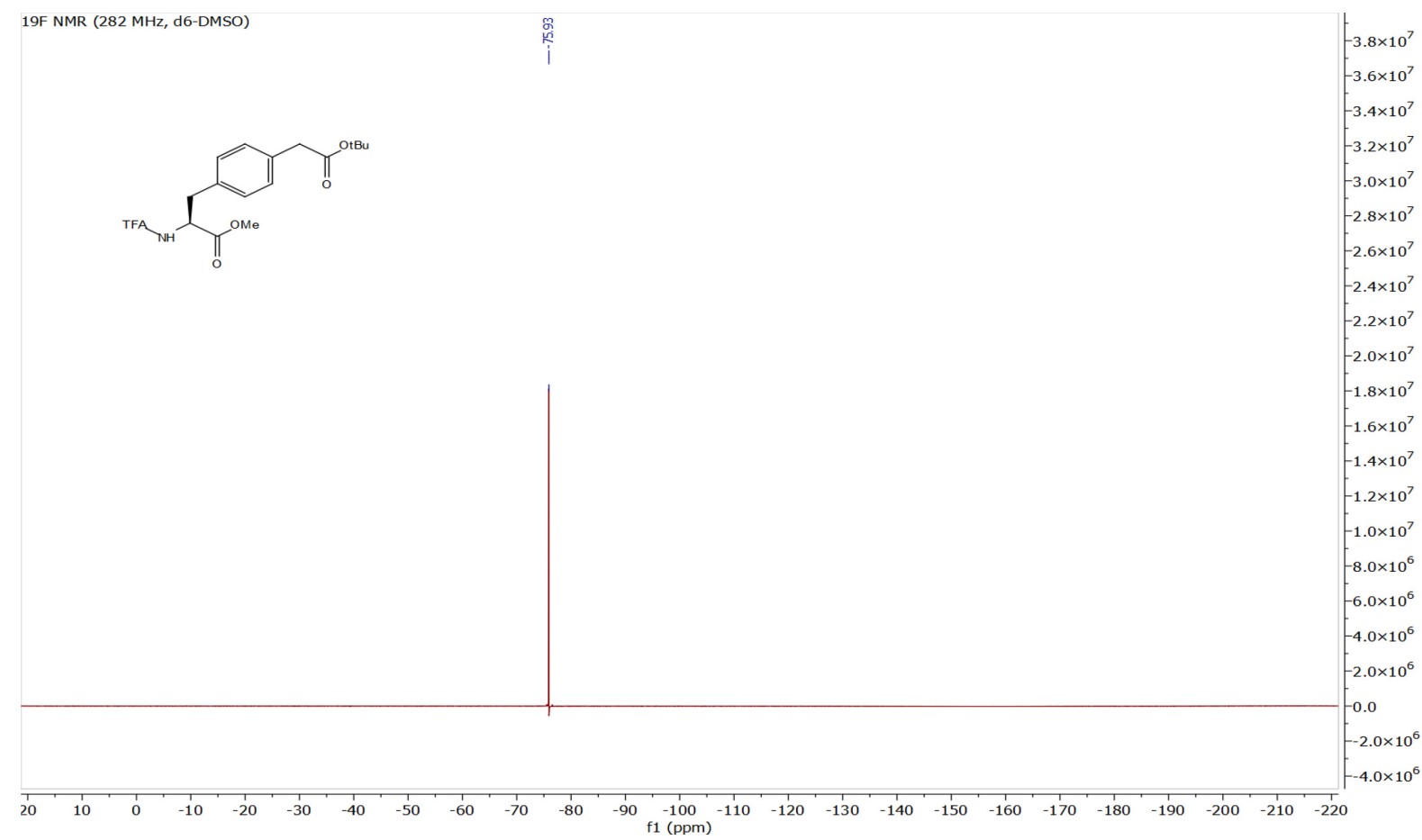

Compound was analyzed by $214 \mathrm{~nm}$ wavelength

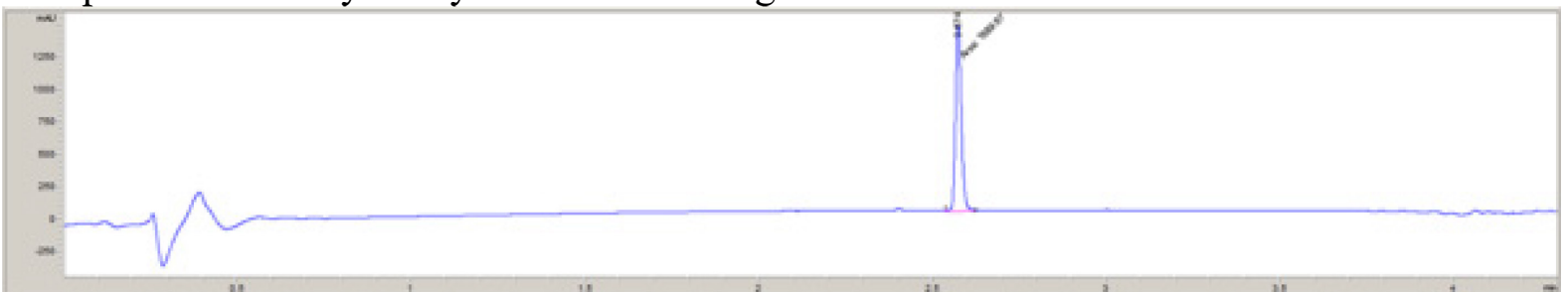


(S)-Methyl-2-(((benzyloxy)carbonyl)amino)-3-(4-(2-(tert-butoxy)-2oxoethyl)phenyl)propanoate (5a)

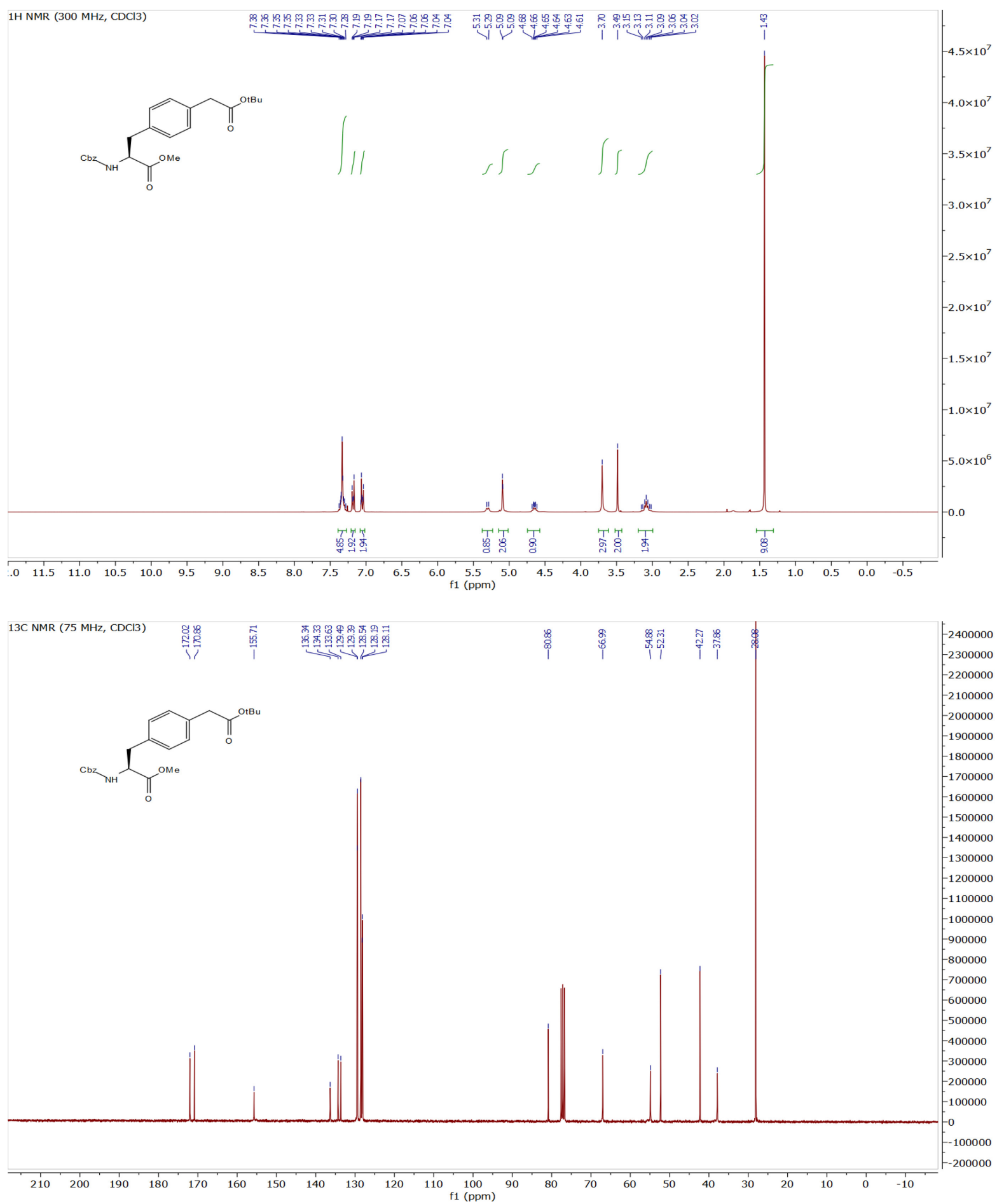

Compound was analyzed by $214 \mathrm{~nm}$ wavelength

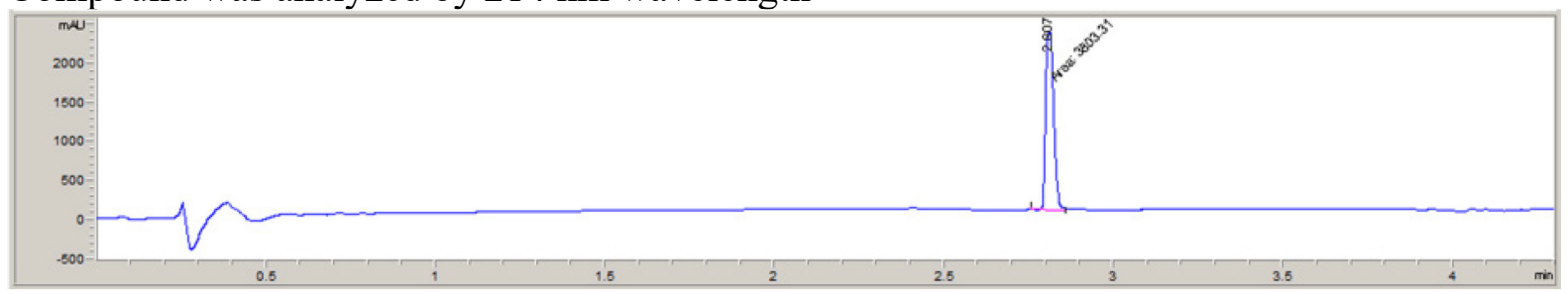


(S)-2-(((Benzyloxy)carbonyl)amino)-3-(4-(2-(tert-butoxy)-2-oxoethyl)phenyl)propanoic acid (5b)

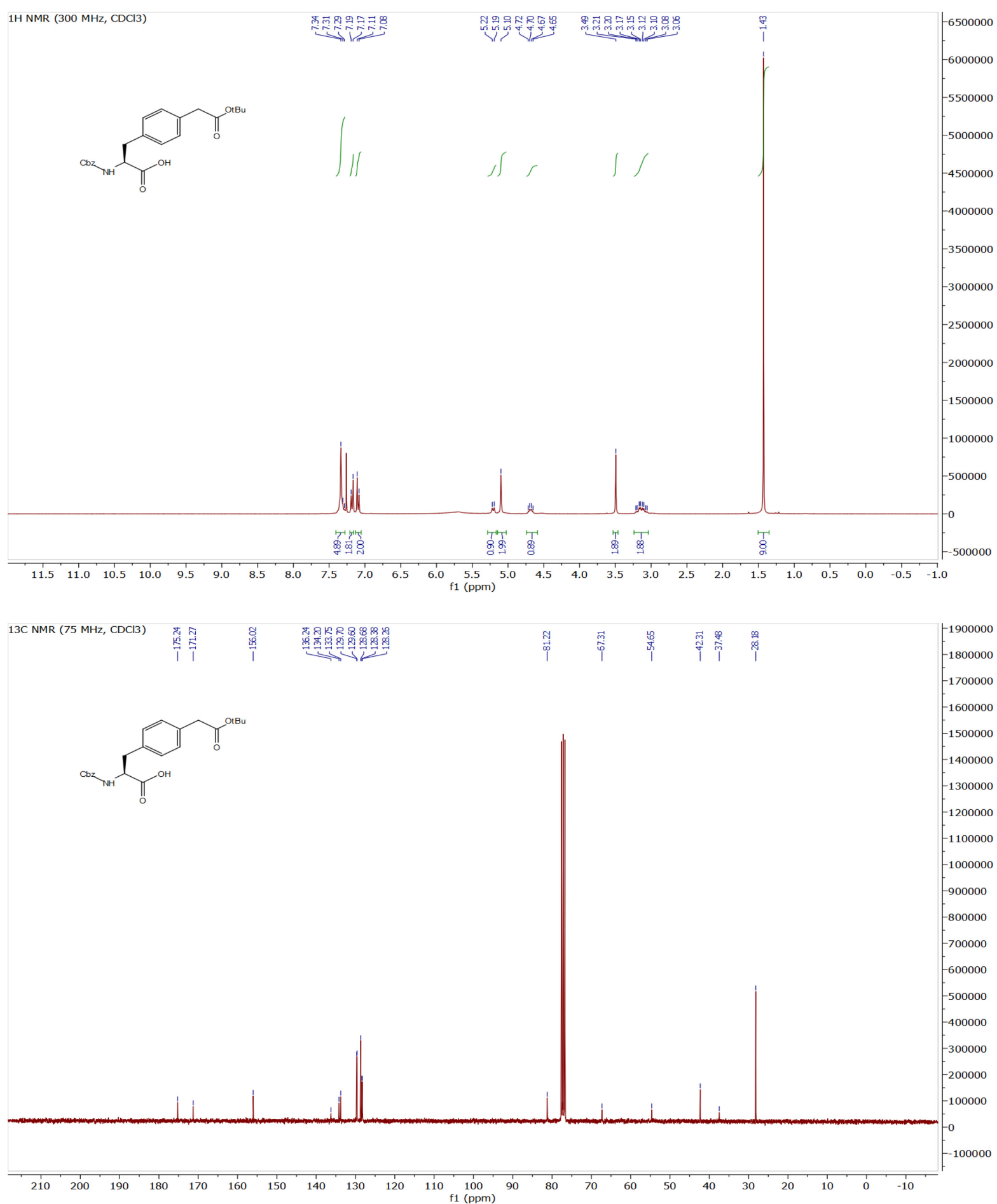

Compound was analyzed by $214 \mathrm{~nm}$ wavelength

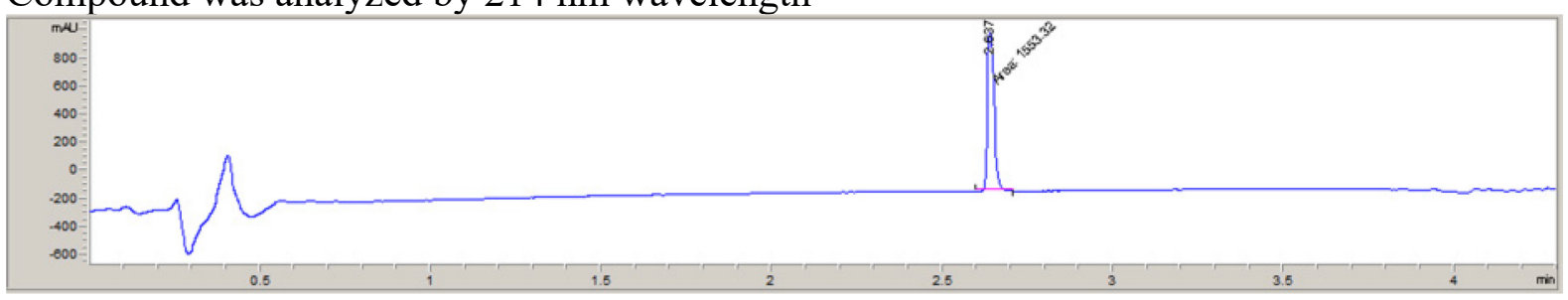


(S)-Methyl-2-((((9H-fluoren-9-yl)methoxy)carbonyl)amino)-3-(4-(2-(tert-butoxy)-2oxoethyl)phenyl)propanoate (5c)
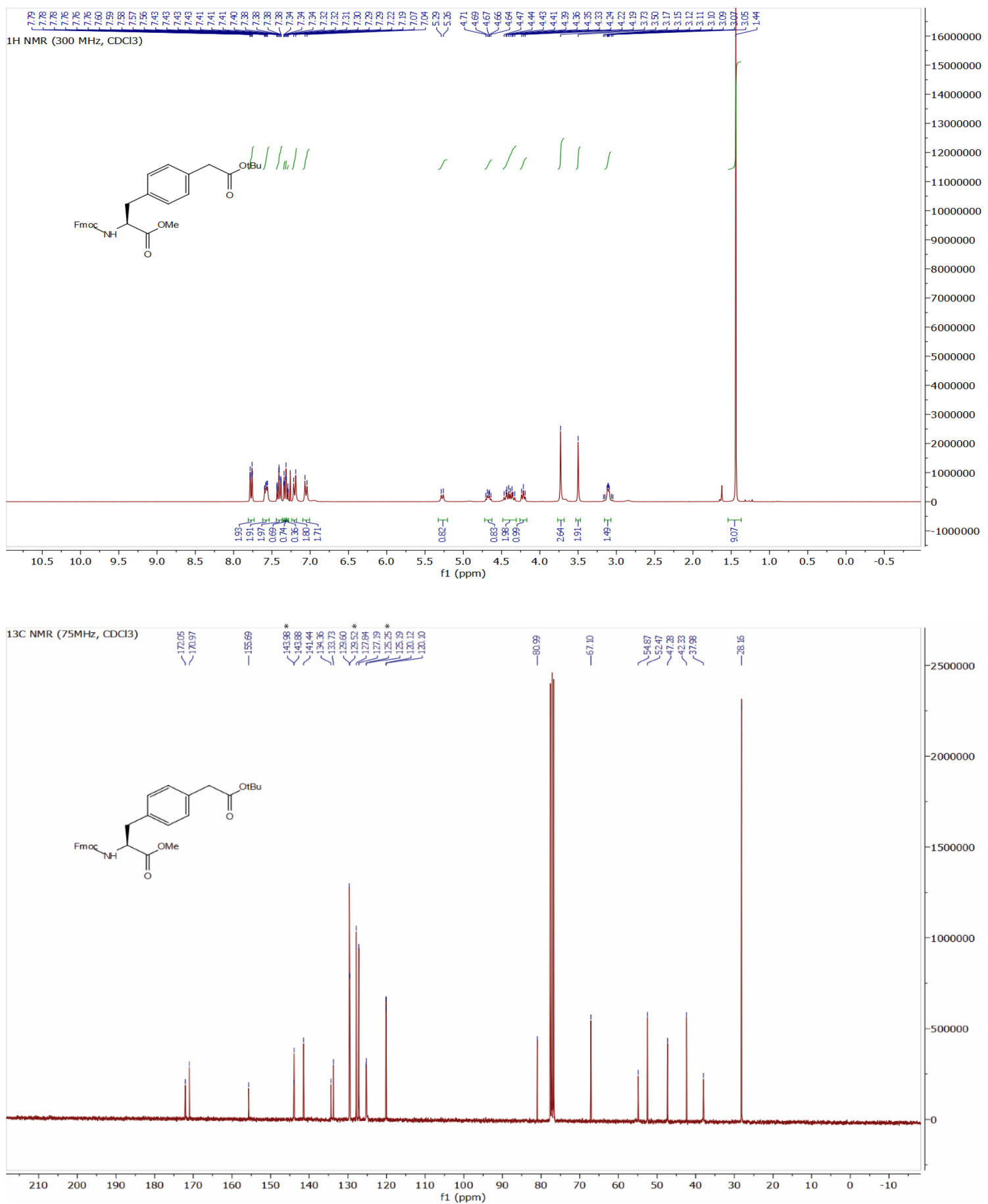

Compound was analyzed by $214 \mathrm{~nm}$ wavelength

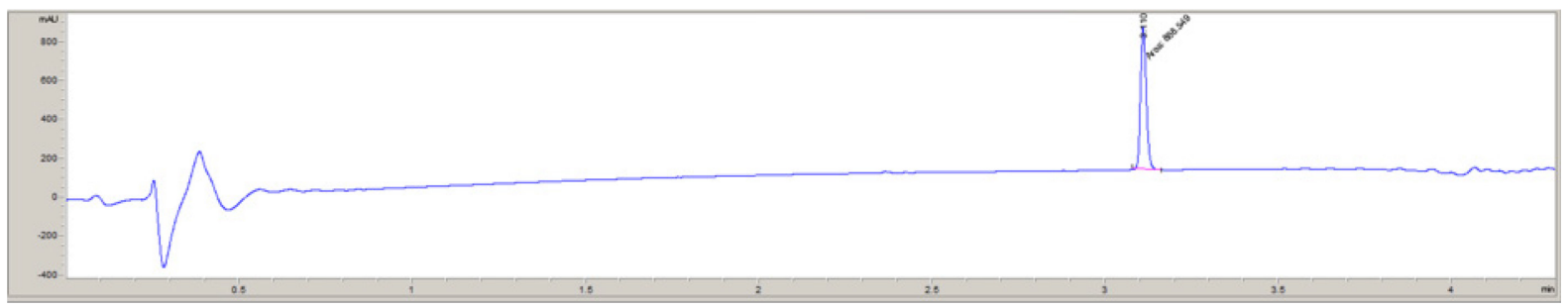


(S)-2-((((9H-Fluoren-9-yl)methoxy)carbonyl)amino)-3-(4-(2-(tert-butoxy)-2oxoethyl)phenyl)propanoic acid (5d)
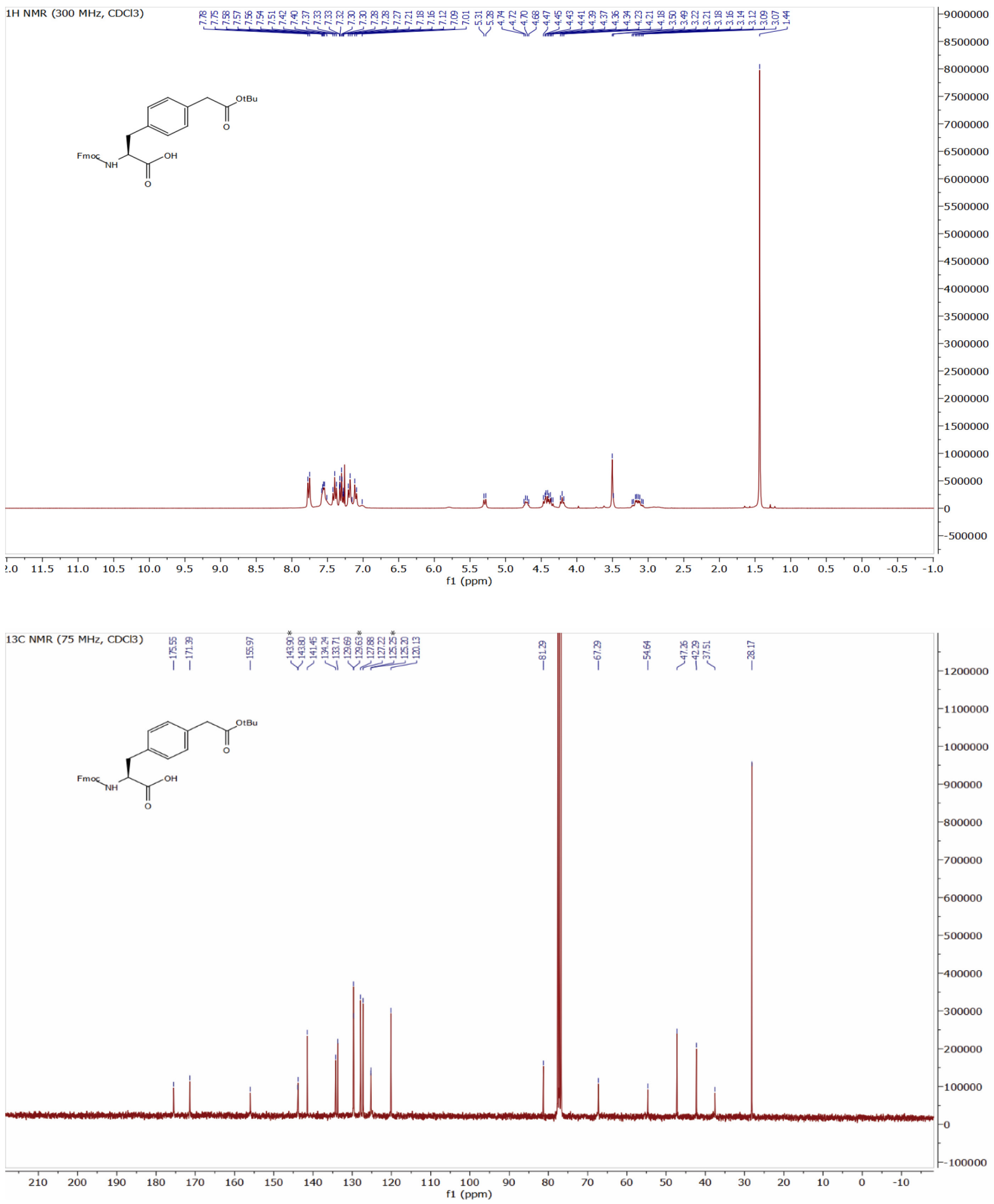

Compound was analyzed by $214 \mathrm{~nm}$ wavelength

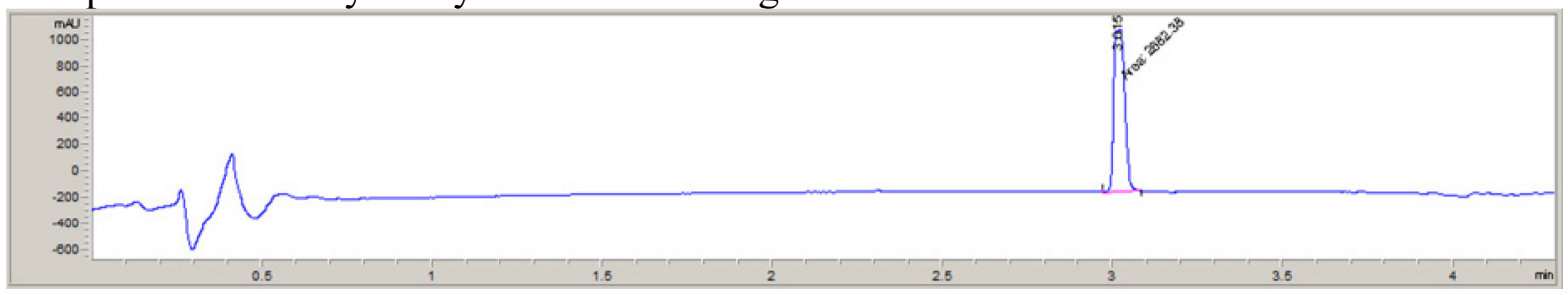


(S)-Methyl-3-(4-(2-(tert-butoxy)-2-oxoethyl)phenyl)-2-((tert-

butoxycarbonyl)amino)propanoate (5e)
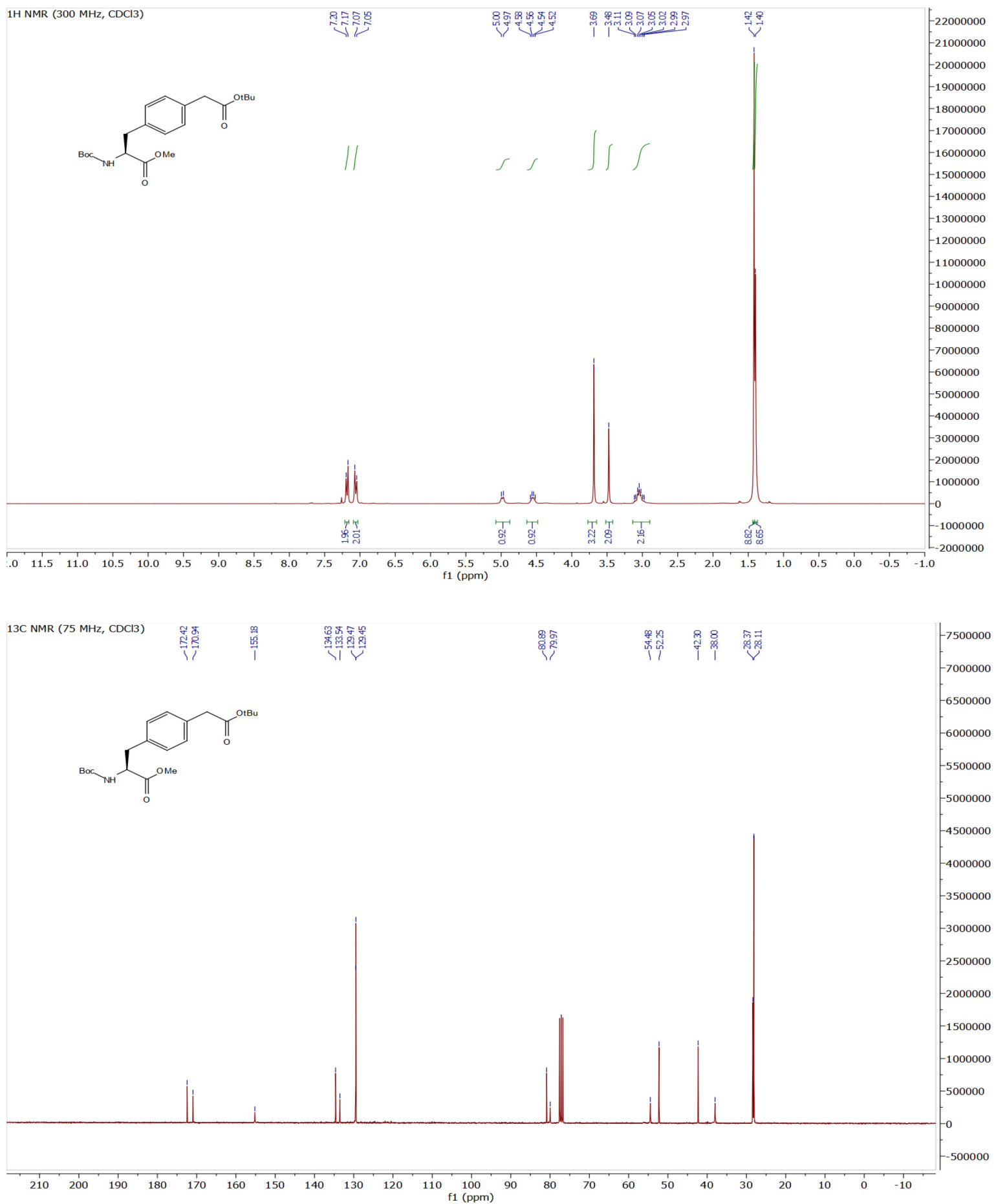

Compound was analyzed by $214 \mathrm{~nm}$ wavelength

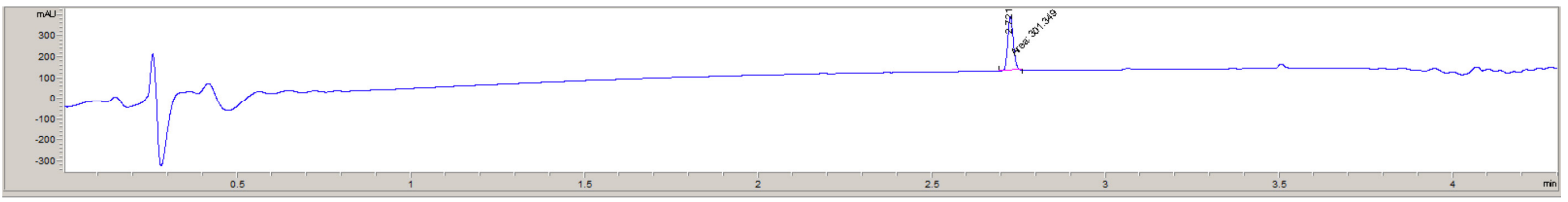


(S)-Methyl 2-amino-3-(4-(2-(tert-butoxy)-2-oxoethyl)phenyl)propanoate (5f)

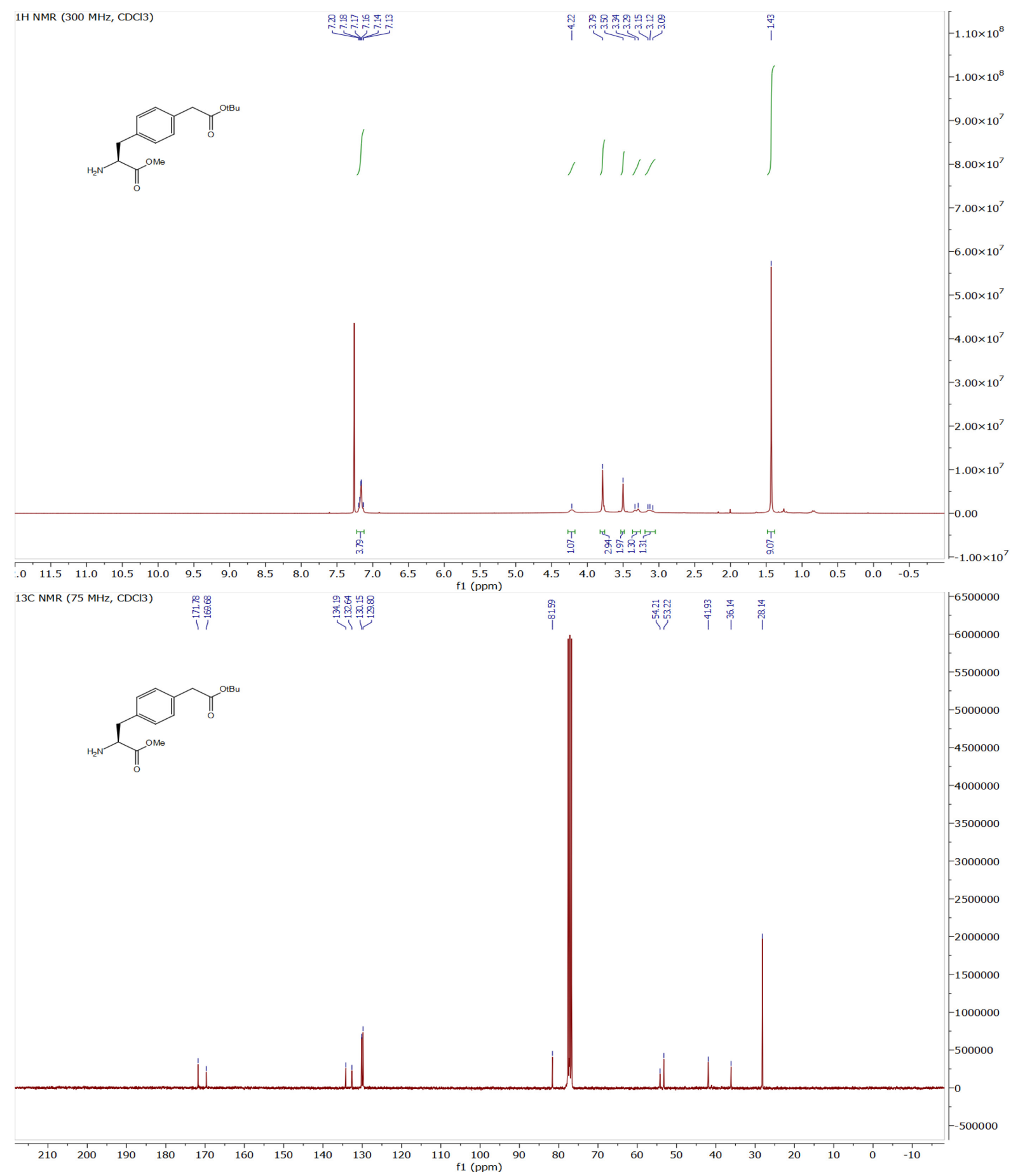

Compound was analyzed by $214 \mathrm{~nm}$ wavelength

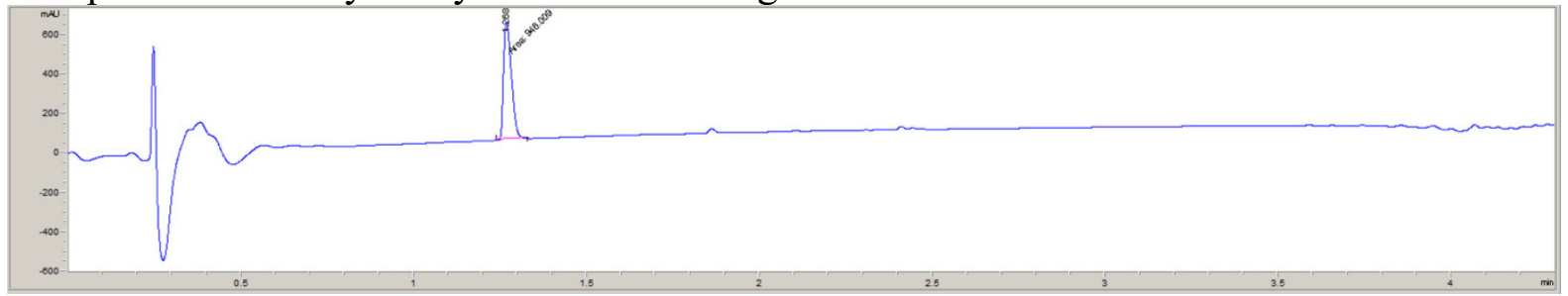


(2S)-Methyl-2-(((benzyloxy)carbonyl)amino)-3-(4-(1-ethoxy-1-oxopropan-2-

\section{yl)phenyl)propanoate (7a)}

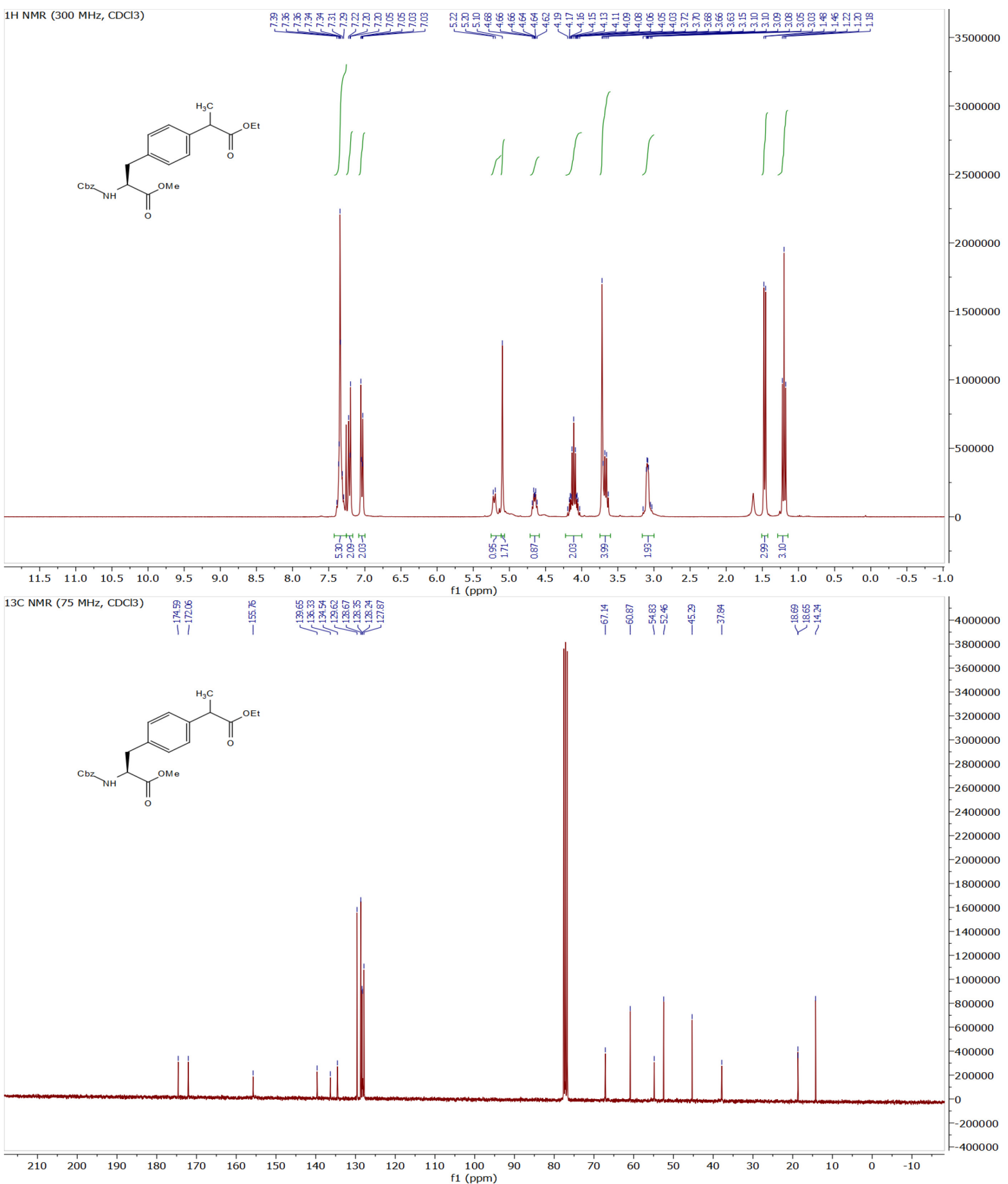

Compound was analyzed by $214 \mathrm{~nm}$ wavelength

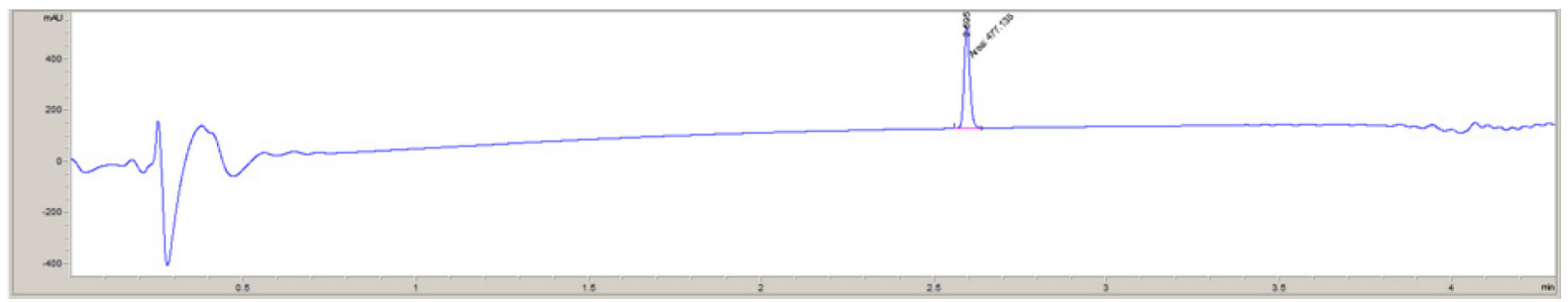


(2S)-Methyl-2-(((benzyloxy)carbonyl)amino)-3-(4-(2-ethoxy-1-fluoro-2oxoethyl)phenyl)propanoate (7b)

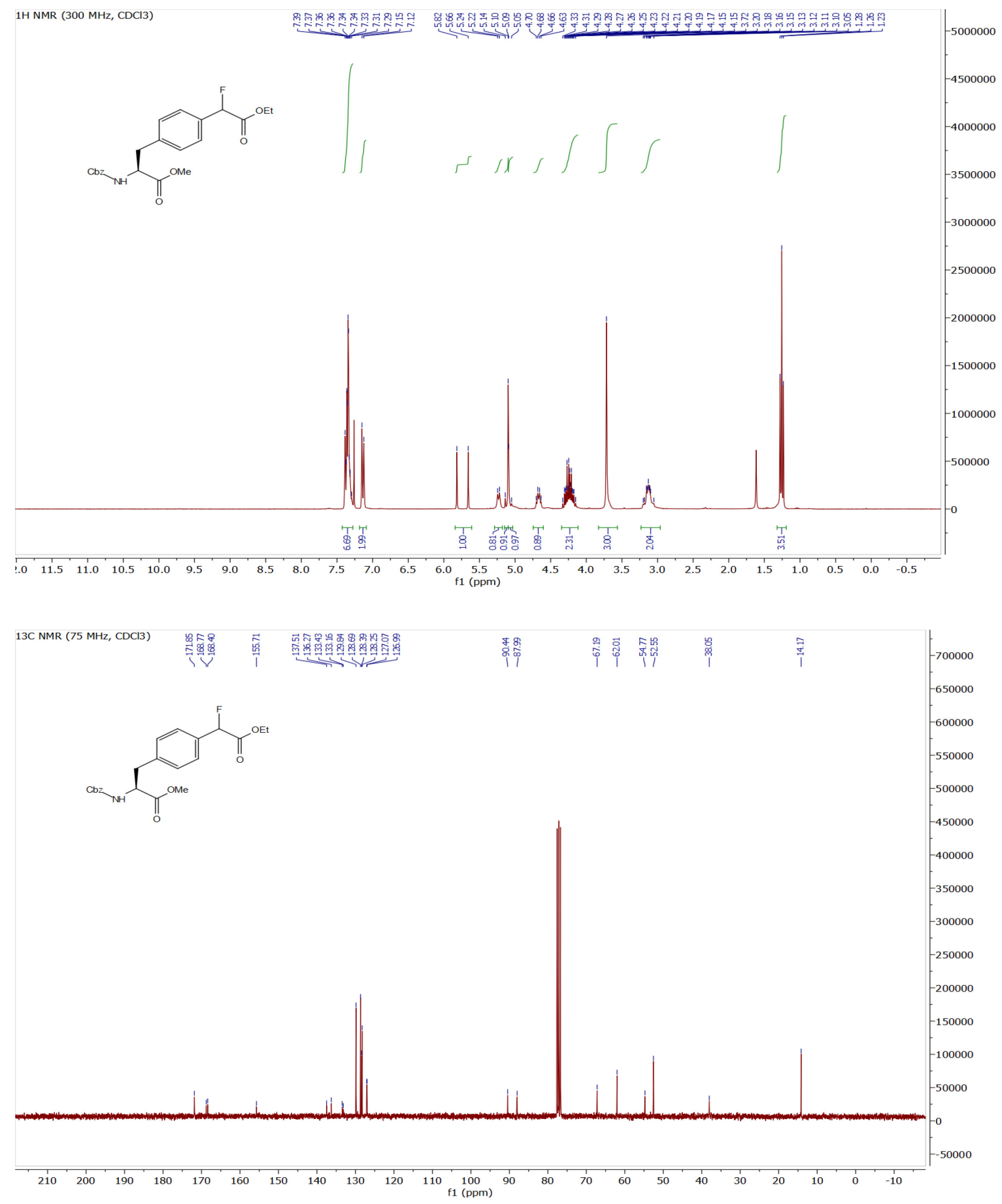




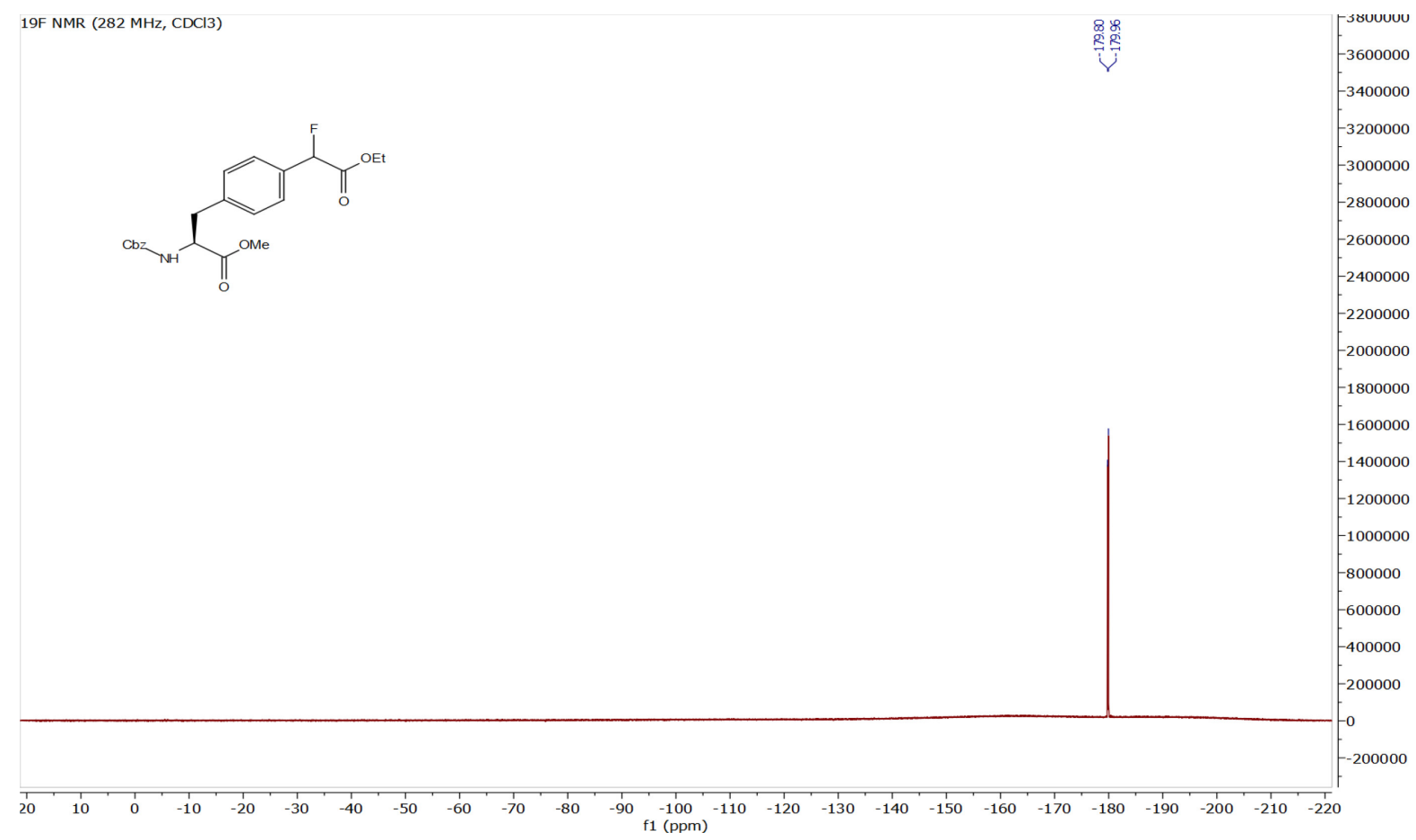

Compound was analyzed by $214 \mathrm{~nm}$ wavelength

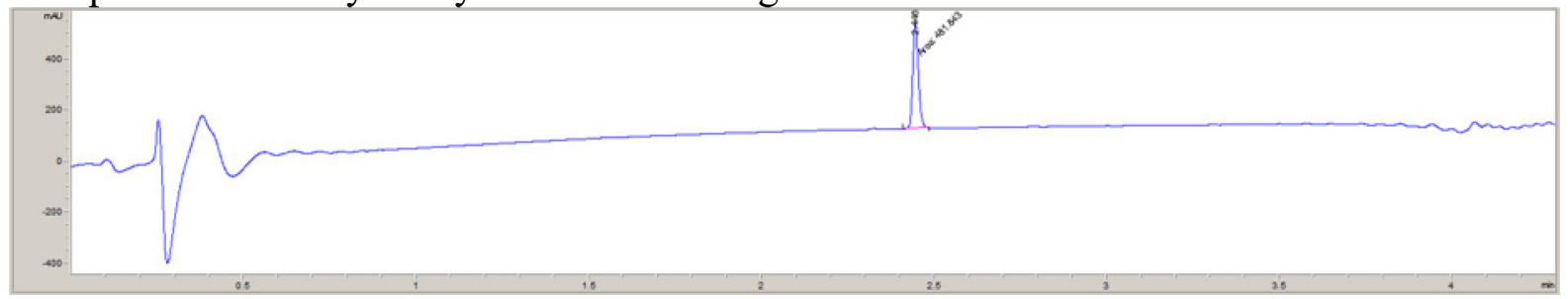


(S)-Methyl 2-(((benzyloxy)carbonyl)amino)-3-(4

((methylsulfonyl)methyl)phenyl)propanoate (7c)

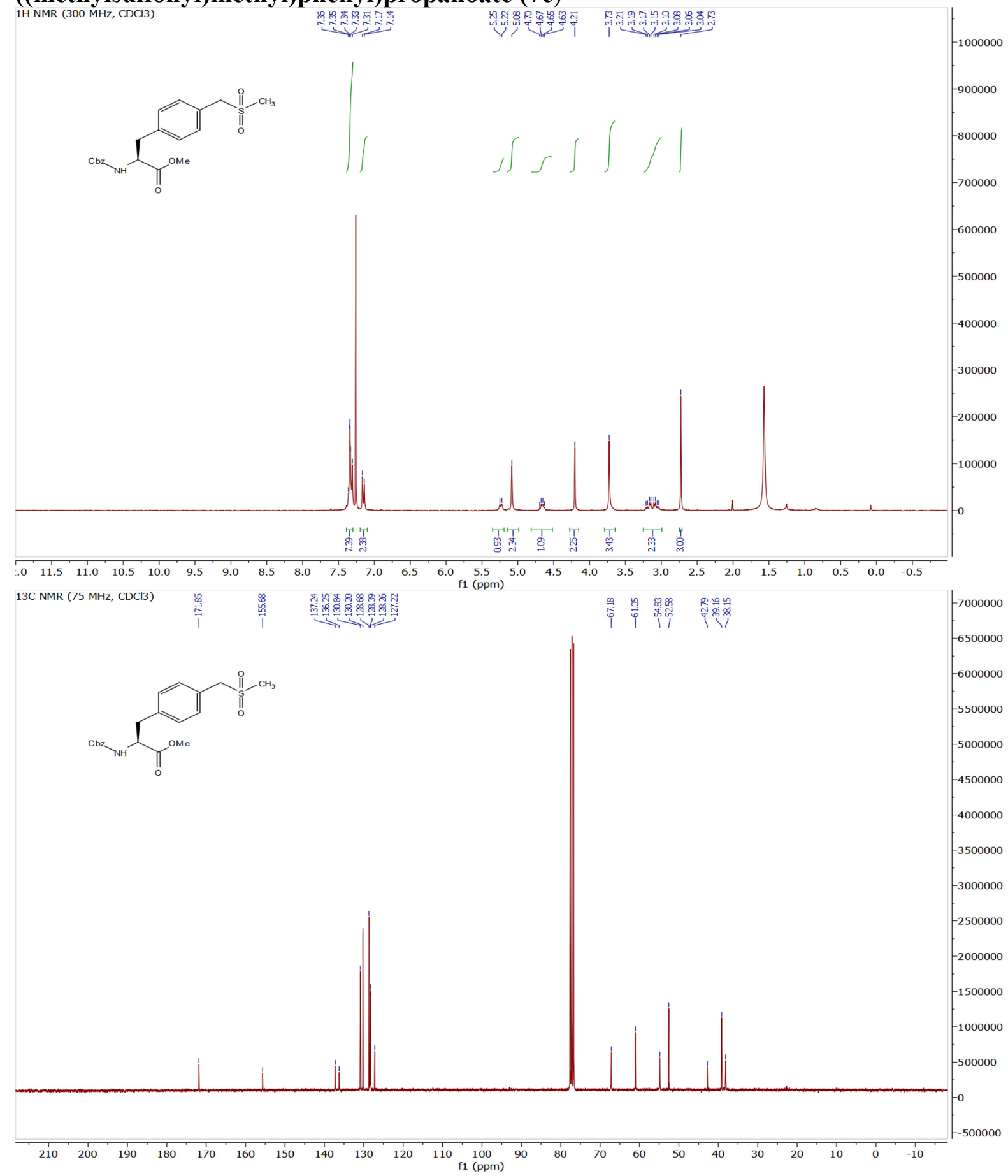

Compound was analyzed by $214 \mathrm{~nm}$ wavelength

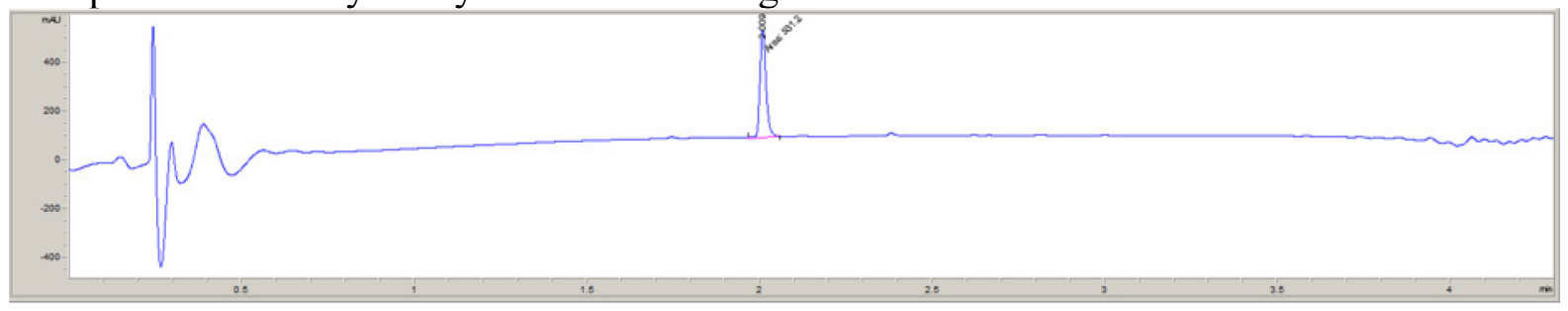


(S)-Methyl 2-(((benzyloxy)carbonyl)amino)-3-(4-

((diethoxyphosphoryl)methyl)phenyl)propanoate (7d)

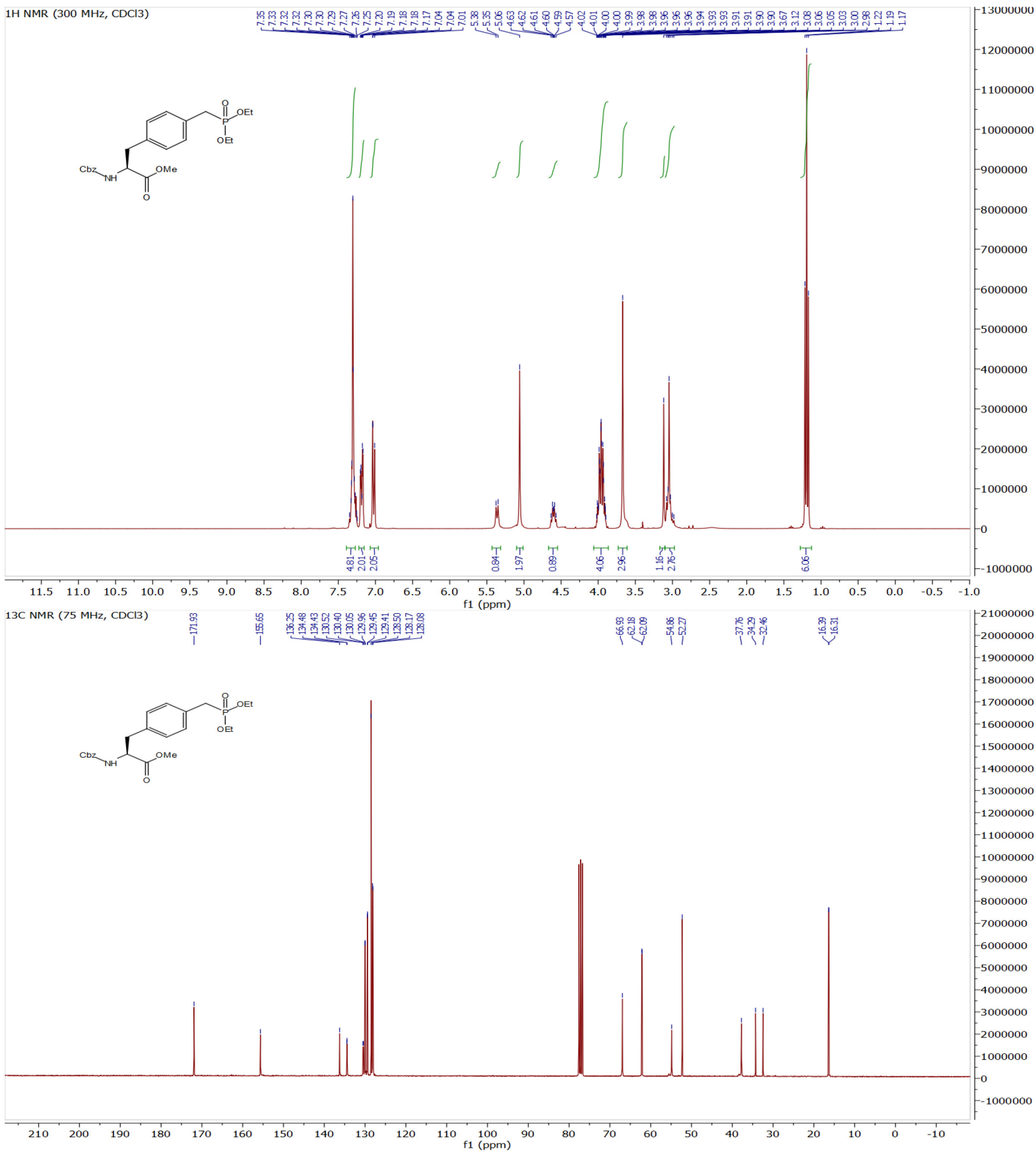




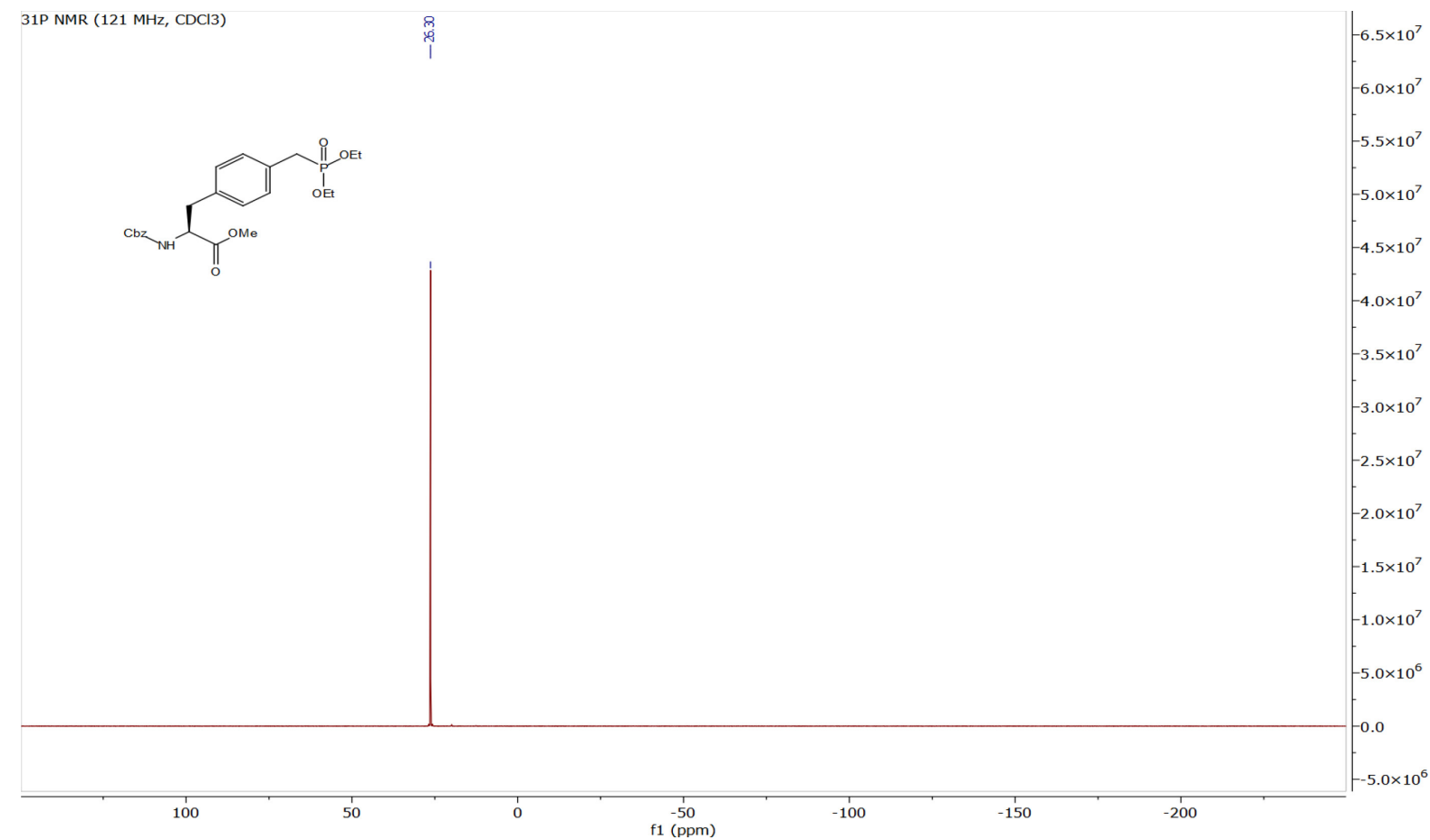

Compound was analyzed by $214 \mathrm{~nm}$ wavelength

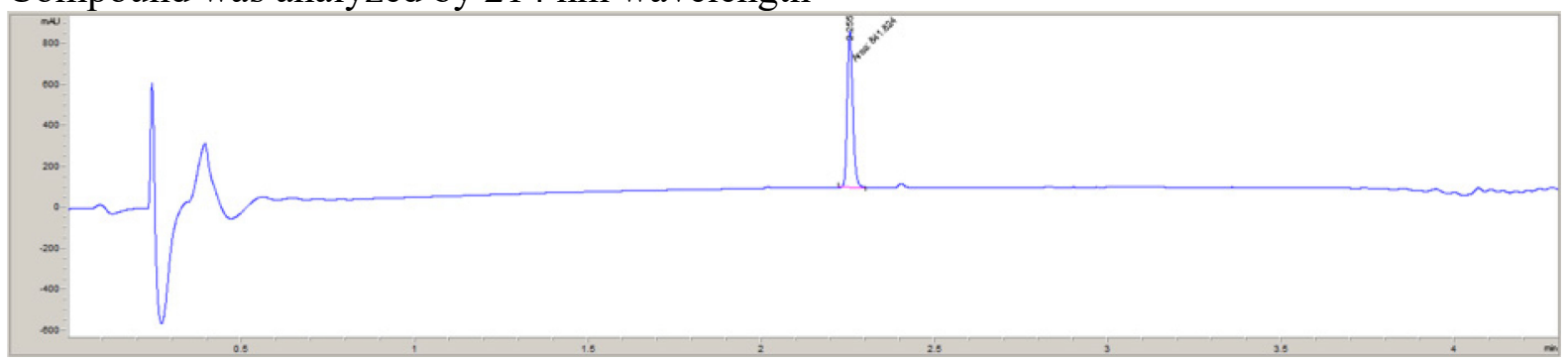


(2S)-Methyl 2-(((benzyloxy)carbonyl)amino)-3-(4-

((ethoxy(methyl)phosphoryl)methyl)phenyl)propanoate (7e)

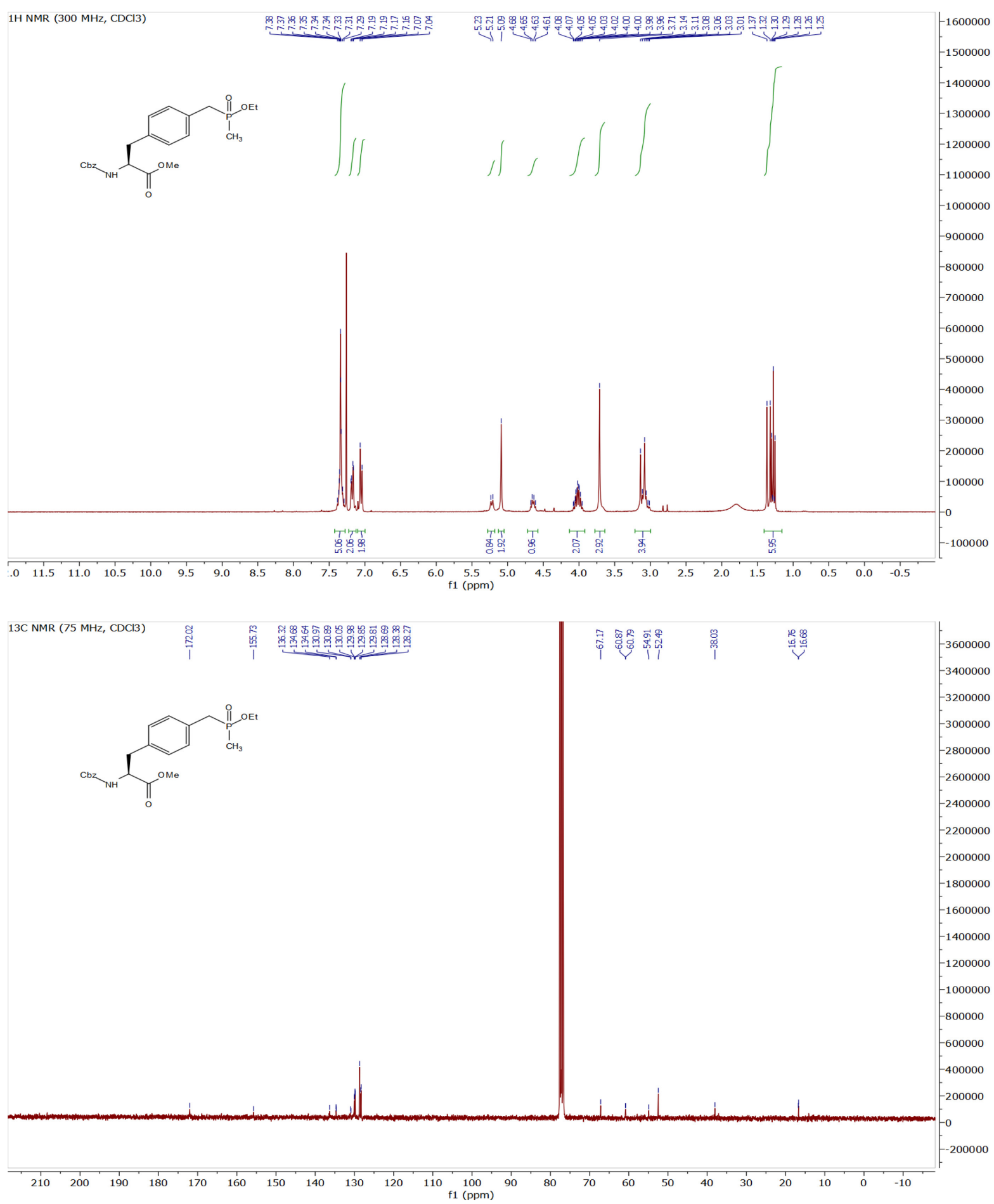




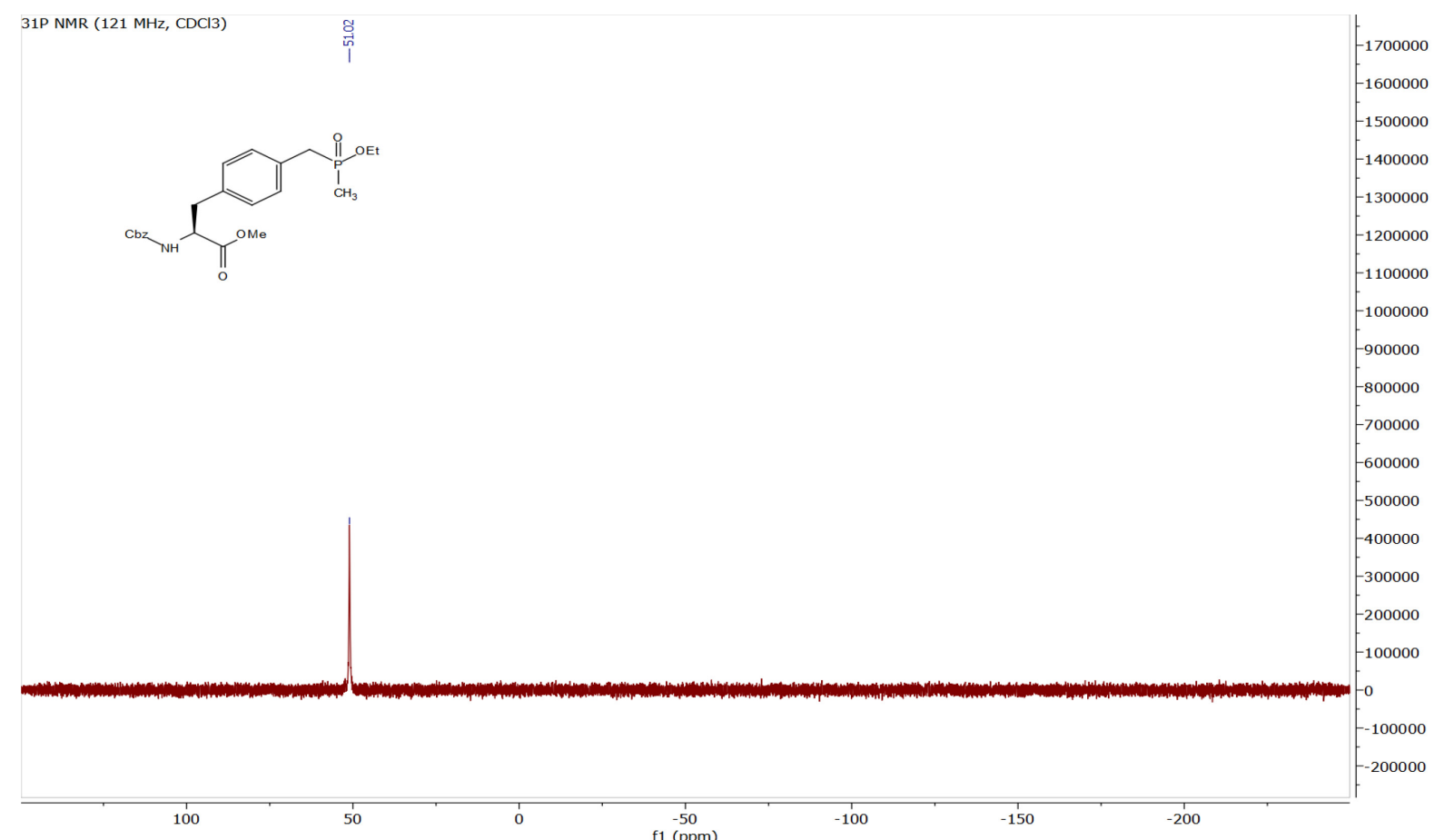

Compound was analyzed by $214 \mathrm{~nm}$ wavelength

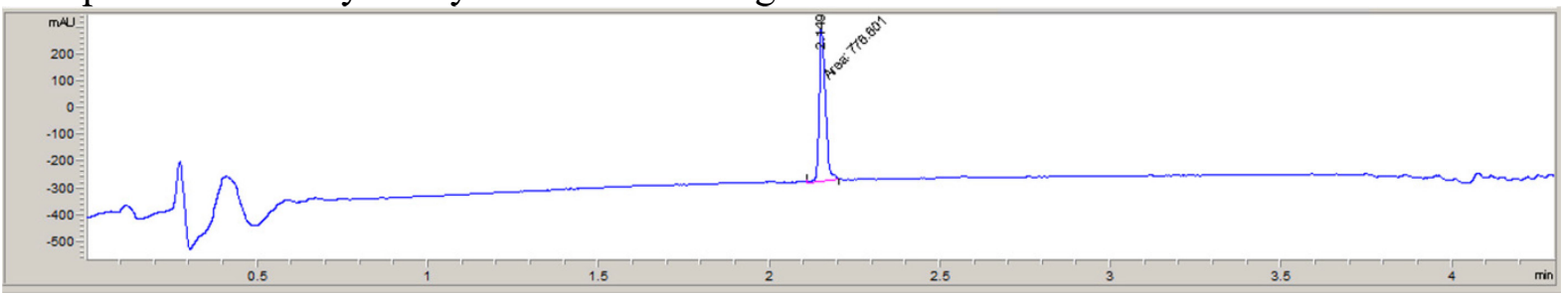


TFA-Phe(4-iodo)-Thr(OtBu)-OMe (8a)

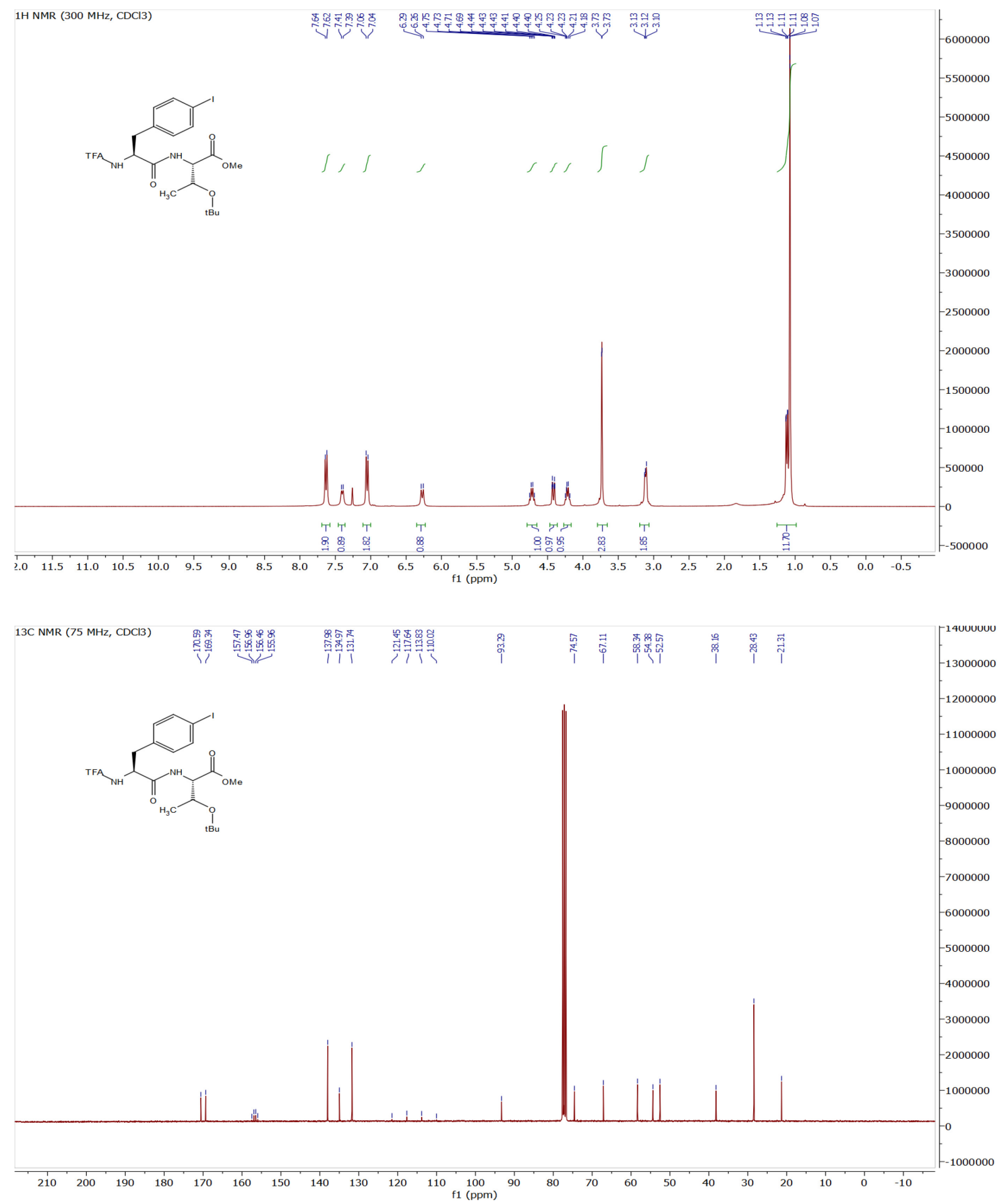




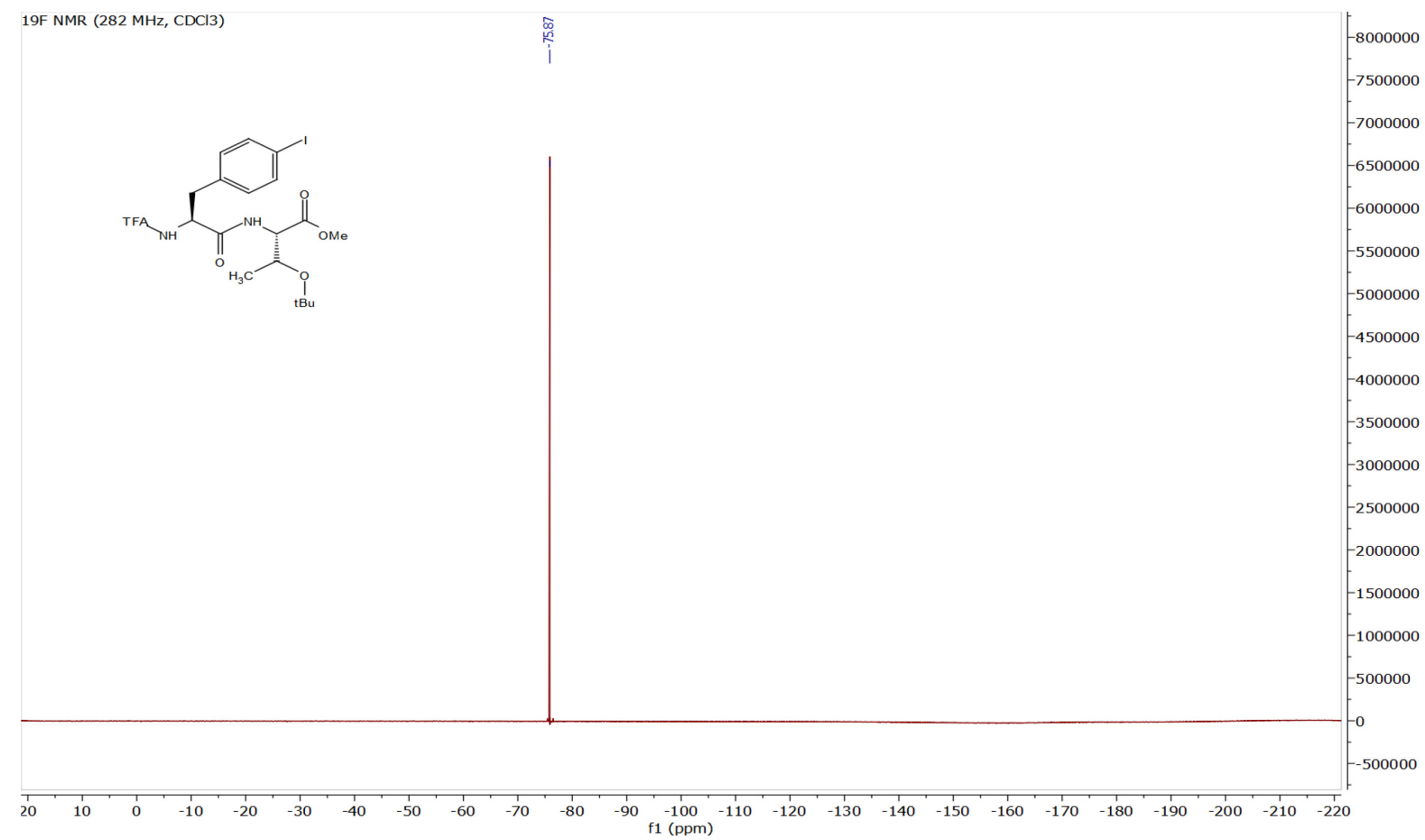

Compound was analyzed by $214 \mathrm{~nm}$ wavelength

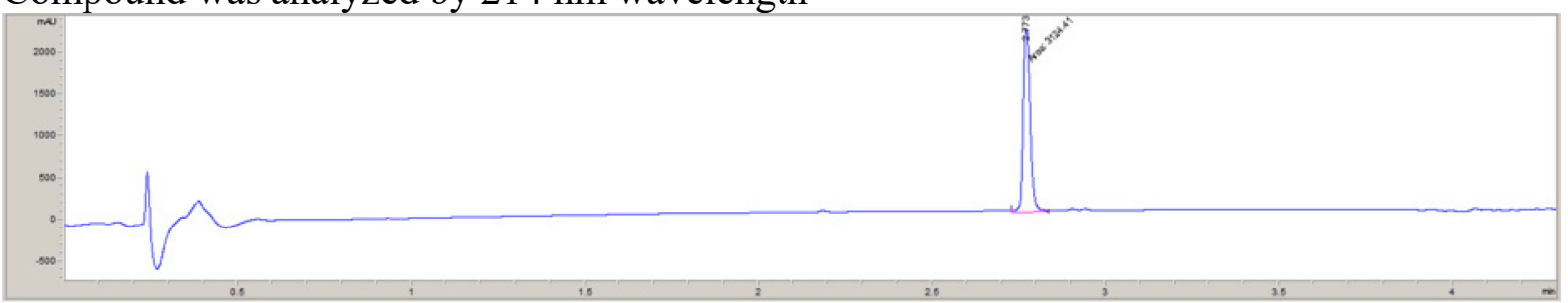


TFA-pCMF(OtBu)-Thr(OtBu)-OMe (9a)
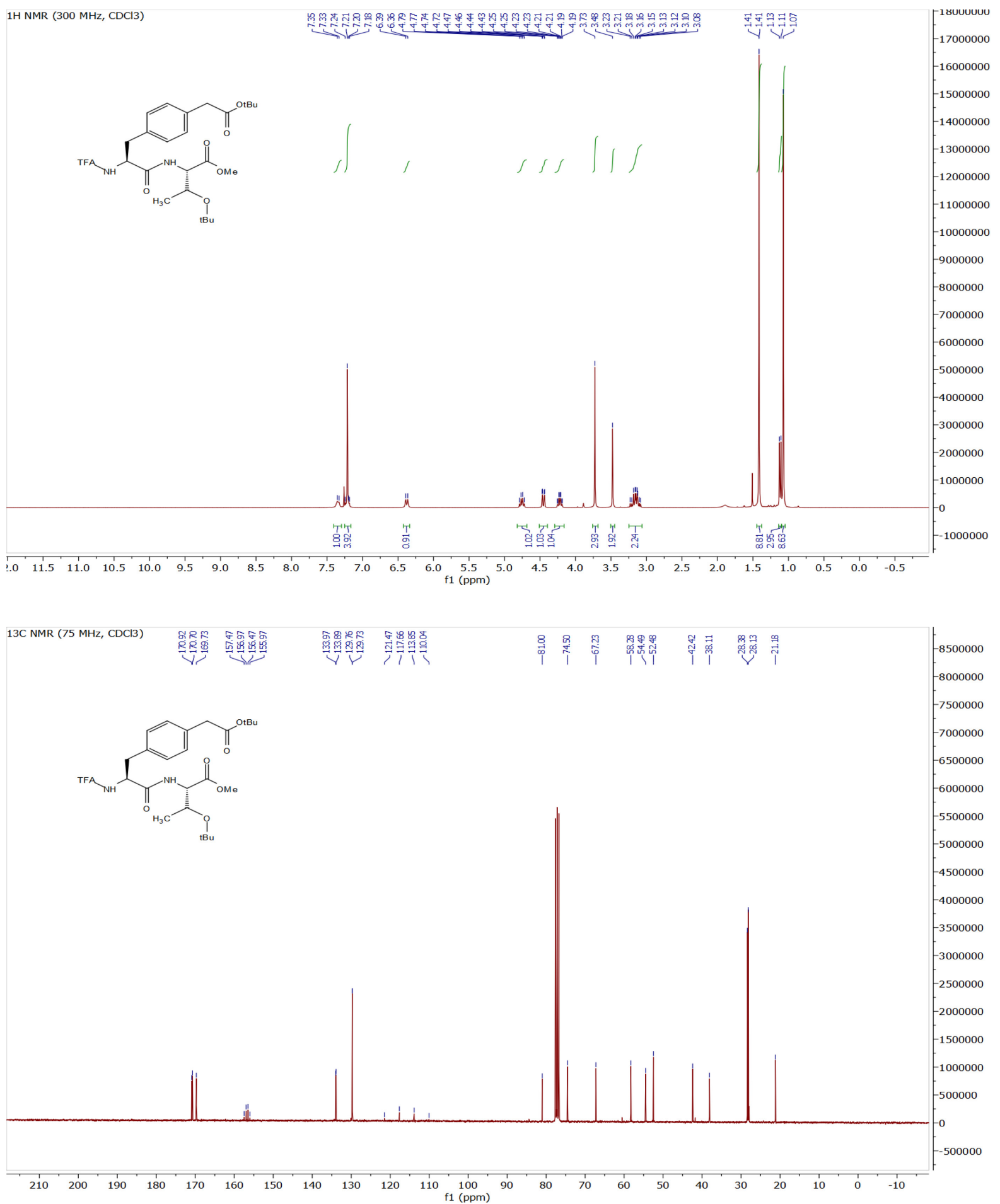


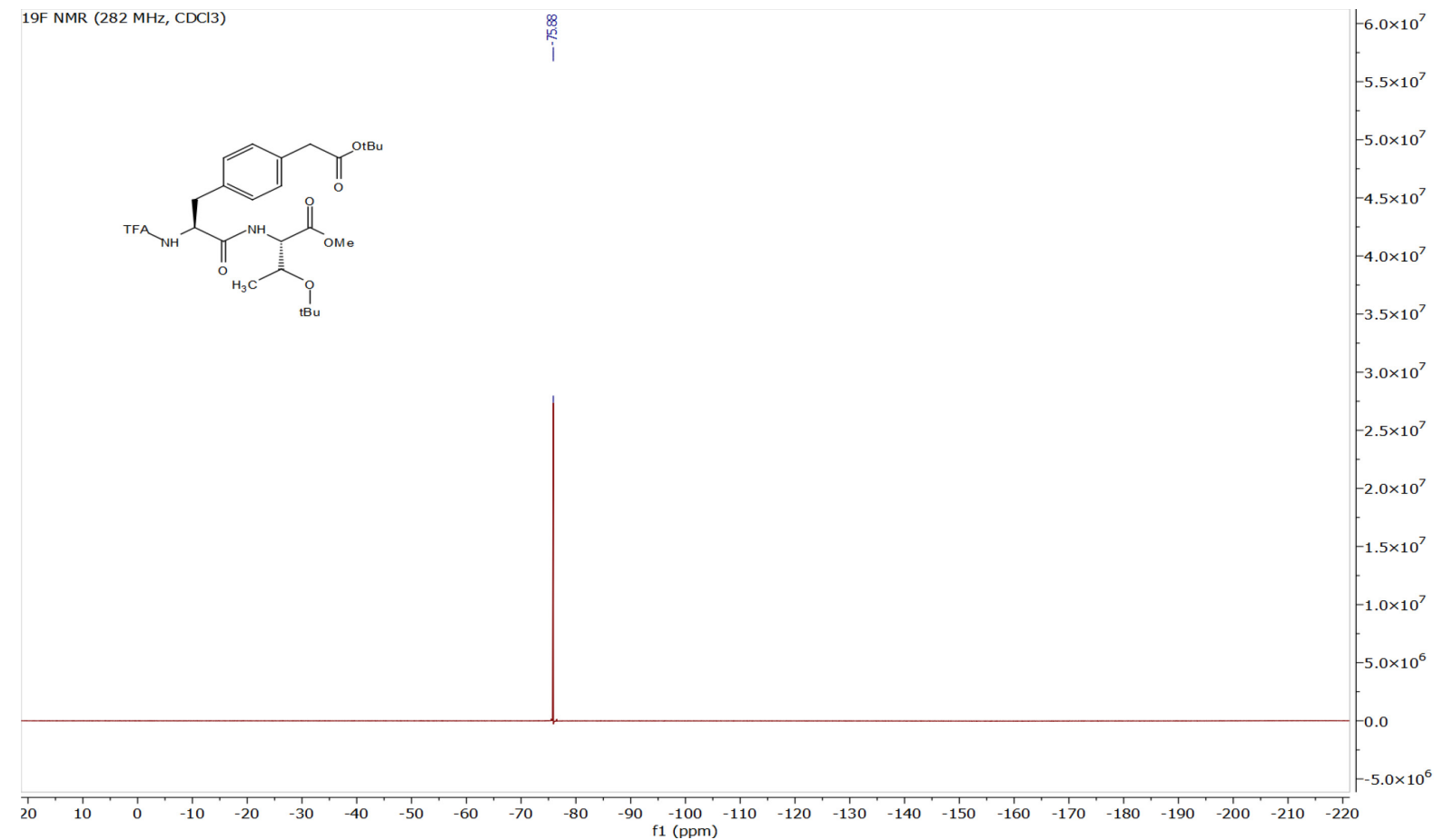

Compound was analyzed by $214 \mathrm{~nm}$ wavelength

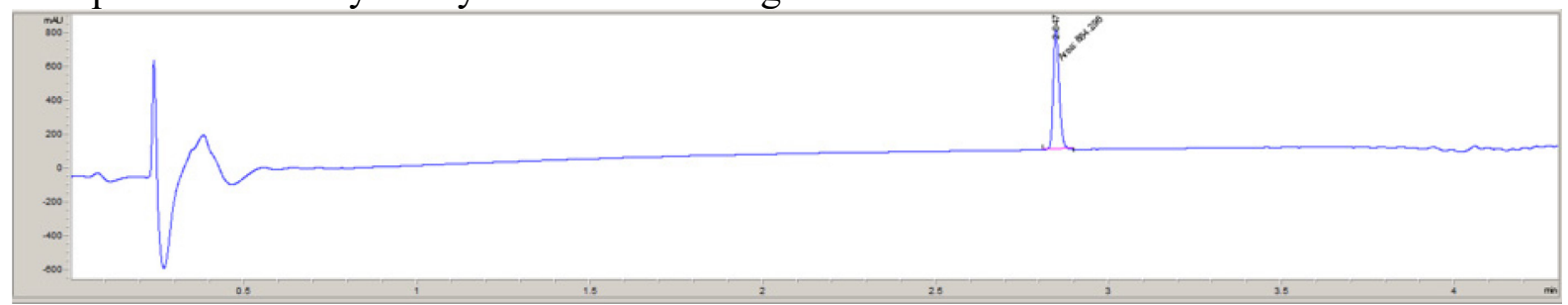




\section{Ac-Glu(OtBu)-Phe(4-iodo)-Thr(OtBu)-NH 2 (8b)}

8.8.8.8. 1 H NMR (300 MHz, d6-DMSO)

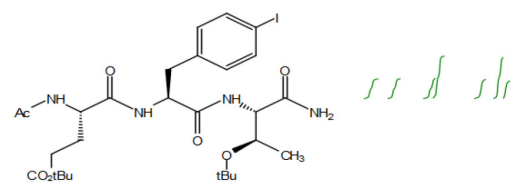
oh
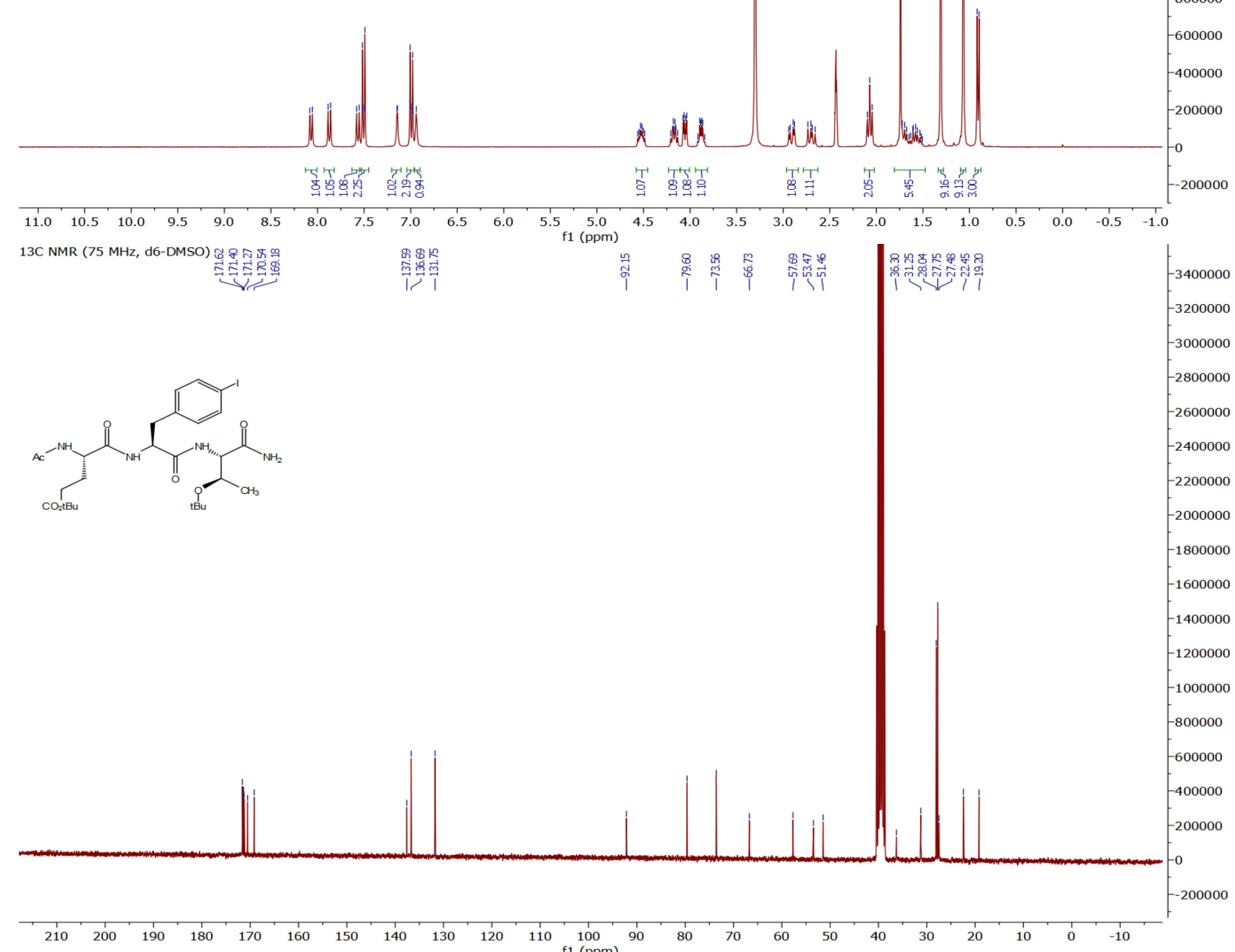

Compound was analyzed by $214 \mathrm{~nm}$ wavelength

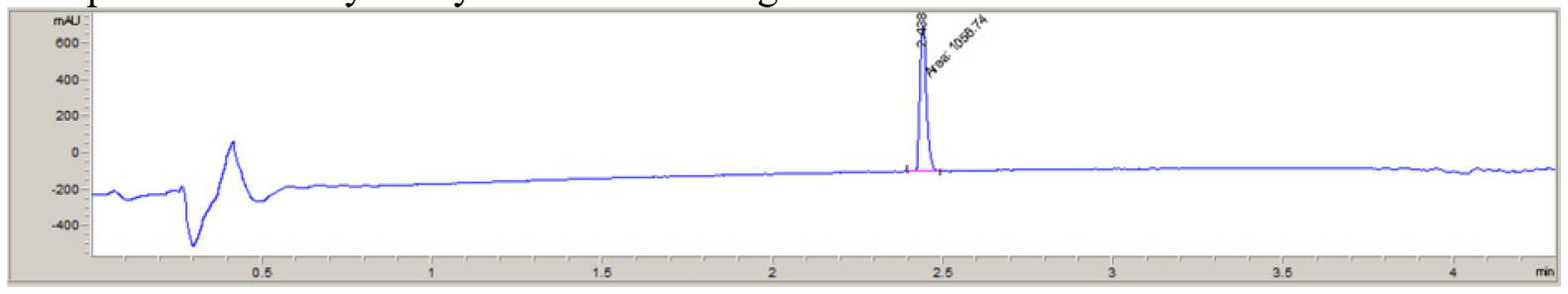




\section{$\operatorname{Ac}-\mathrm{Glu}(\mathrm{O} t \mathrm{Bu})-p \mathrm{CMF}(\mathrm{O} t \mathrm{Bu})-\mathrm{Thr}(\mathrm{O} t \mathrm{Bu})-\mathrm{NH}_{2}(9 \mathrm{~b})$}

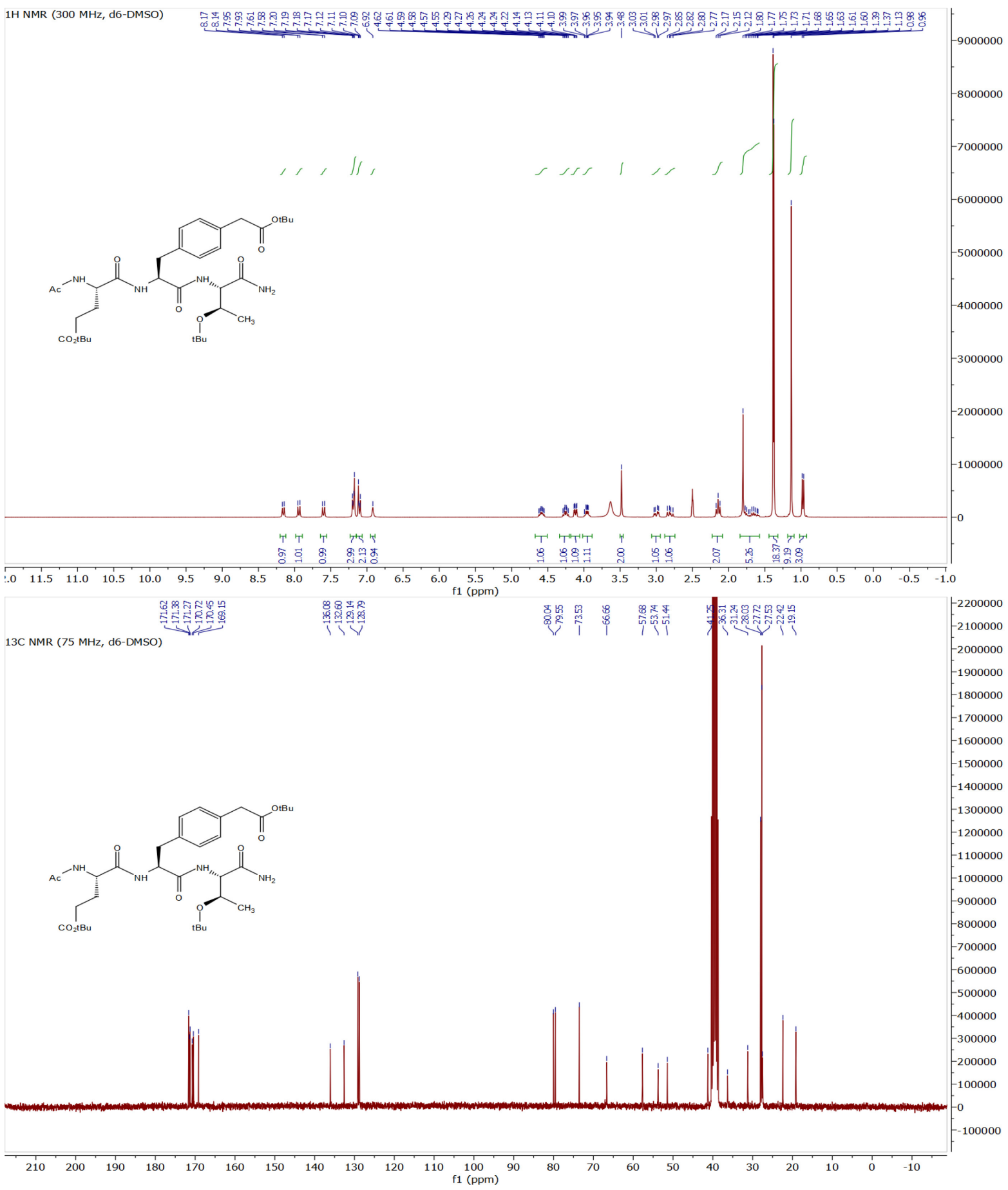

Compound was analyzed by $214 \mathrm{~nm}$ wavelength

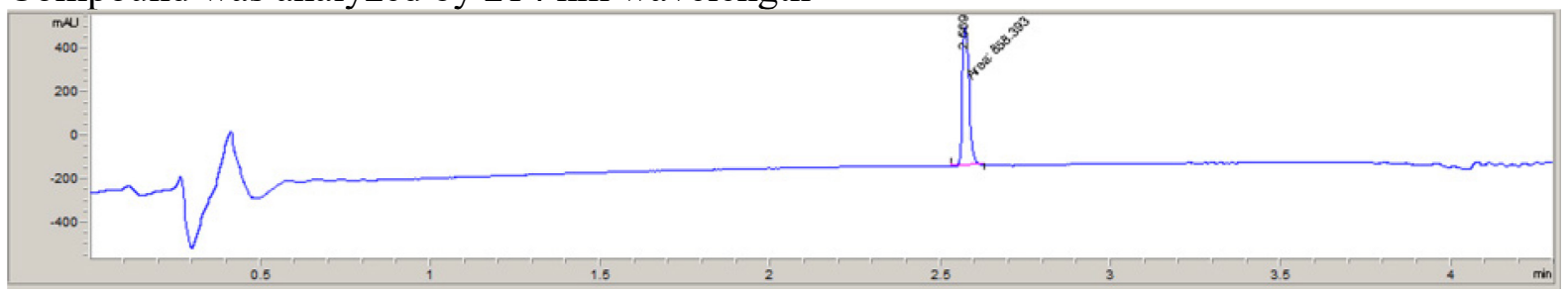




\section{Ac-Met-Phe(4-iodo)-Thr(OtBu)-NH2 (8c)}

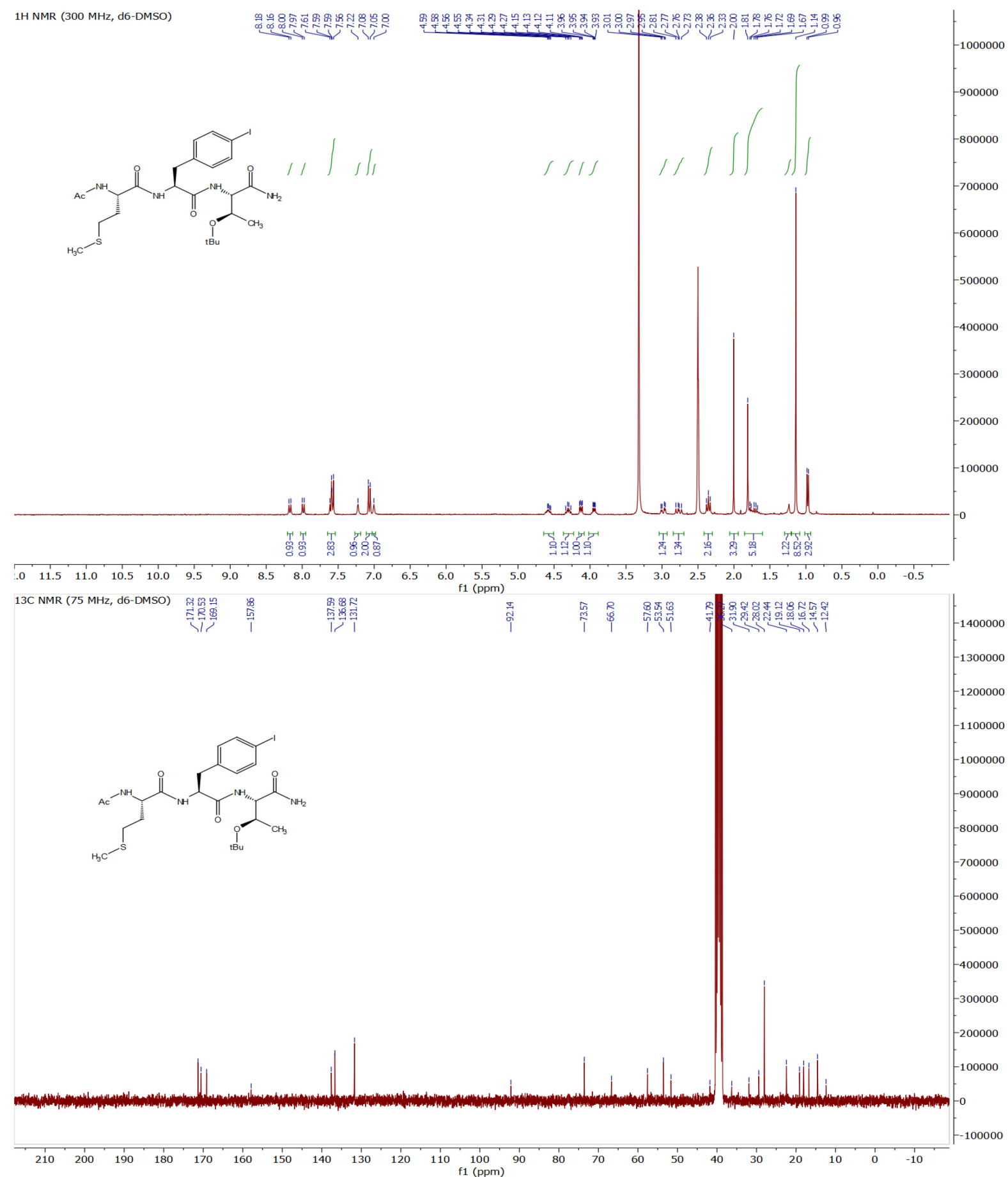

Compound was analyzed by $214 \mathrm{~nm}$ wavelength

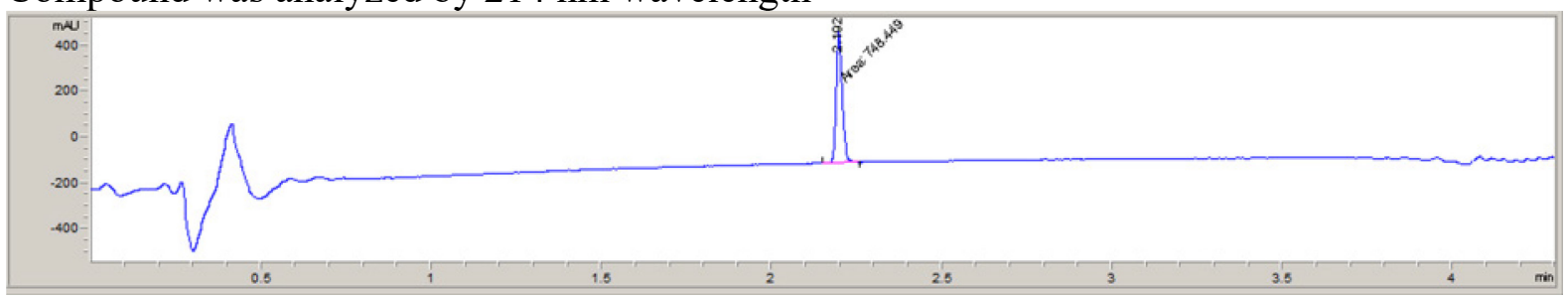




\section{Ac-Met- $p \mathrm{CMF}(\mathrm{O} t \mathrm{Bu})-\mathrm{Thr}(\mathrm{O} t \mathrm{Bu})-\mathrm{NH}_{2}(9 \mathrm{c})$}

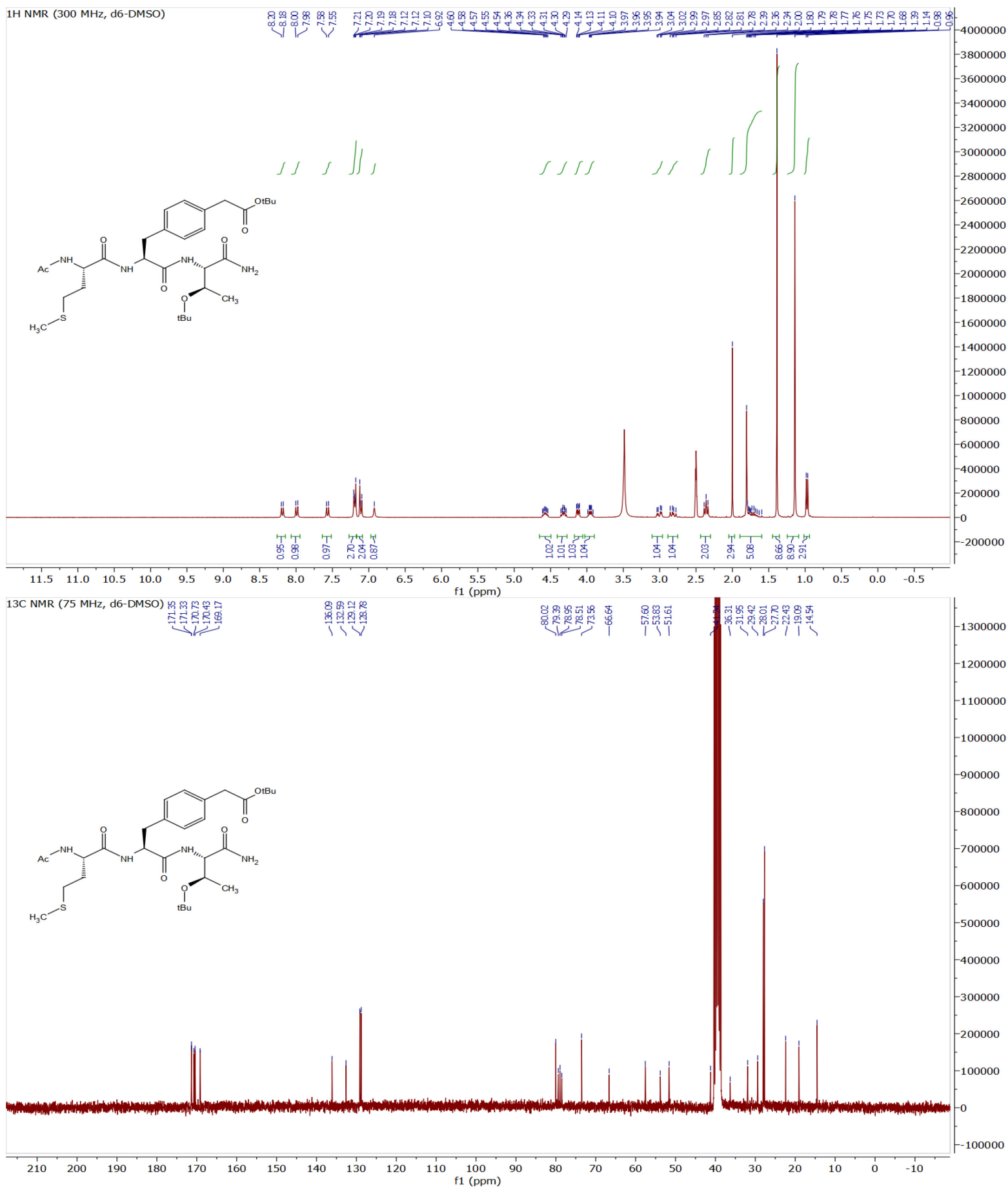

Compound was analyzed by $214 \mathrm{~nm}$ wavelength

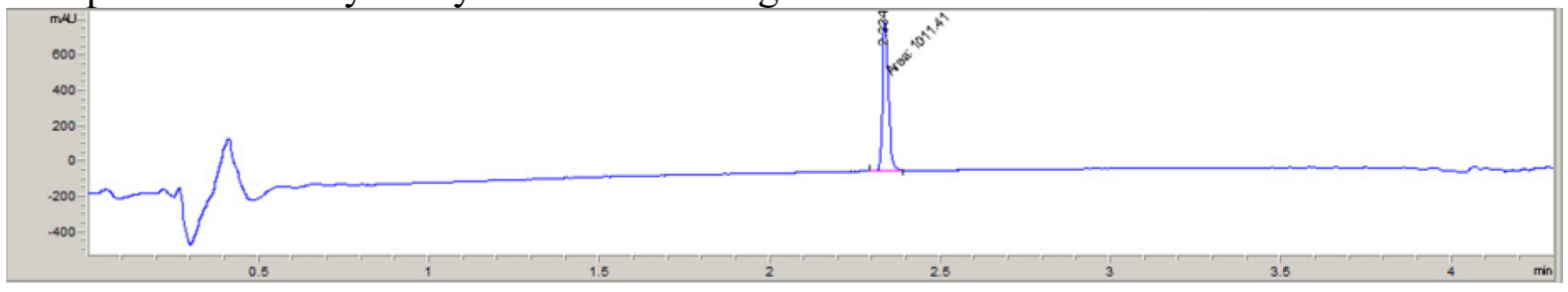




\section{$\operatorname{Ac}-\mathrm{Tyr}(\mathrm{O} t \mathrm{Bu})-\mathrm{Phe}(4-\mathrm{Iodo})-\mathrm{Thr}(\mathrm{O} t \mathrm{Bu})-\mathrm{NH}_{2}$ (8d)}

1H NMR (300 MHz, d6-DMSO)

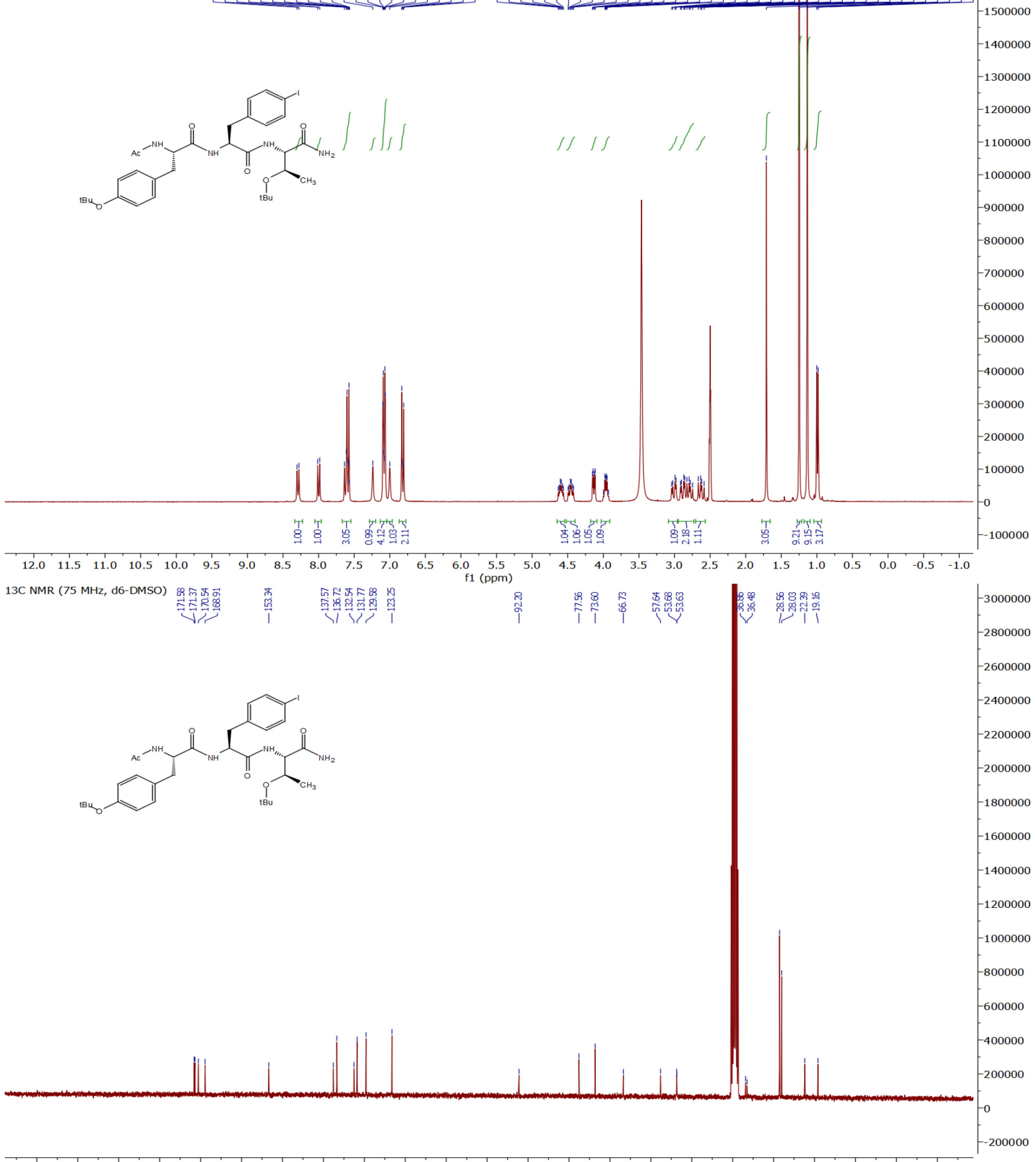

Compound was analyzed by $214 \mathrm{~nm}$ wavelength

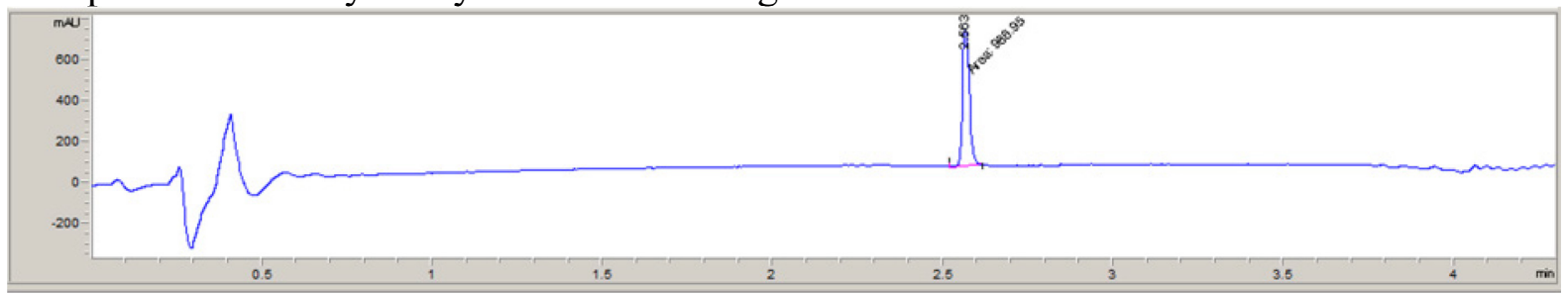




\section{$\operatorname{Ac}-\mathrm{Tyr}(\mathrm{O} t \mathrm{Bu})-p \mathrm{CMF}(\mathrm{O} t \mathrm{Bu})-\mathrm{Thr}(\mathrm{O} t \mathrm{Bu})-\mathrm{NH}_{2}(9 \mathrm{~d})$}

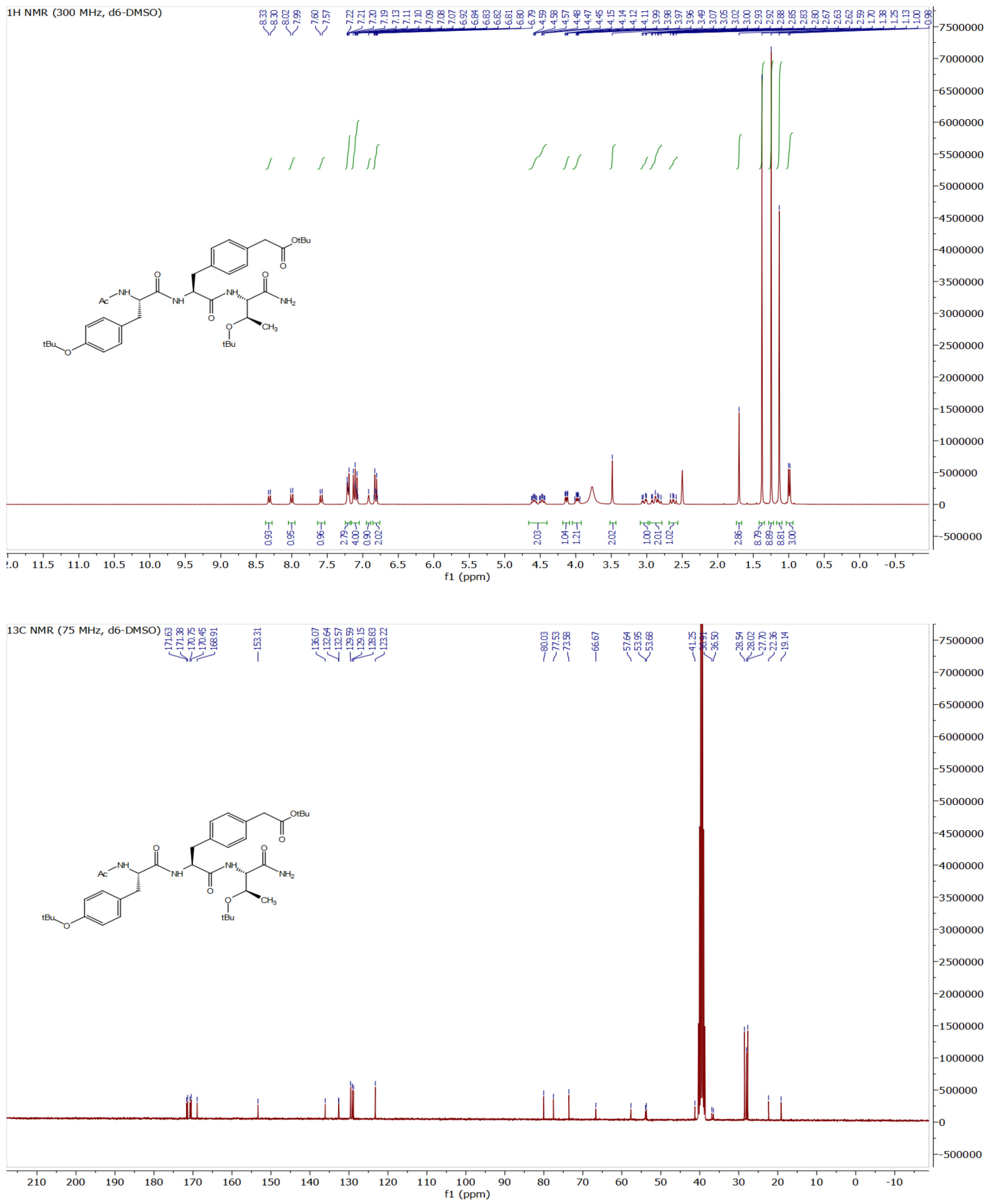

Compound was analyzed by $214 \mathrm{~nm}$ wavelength

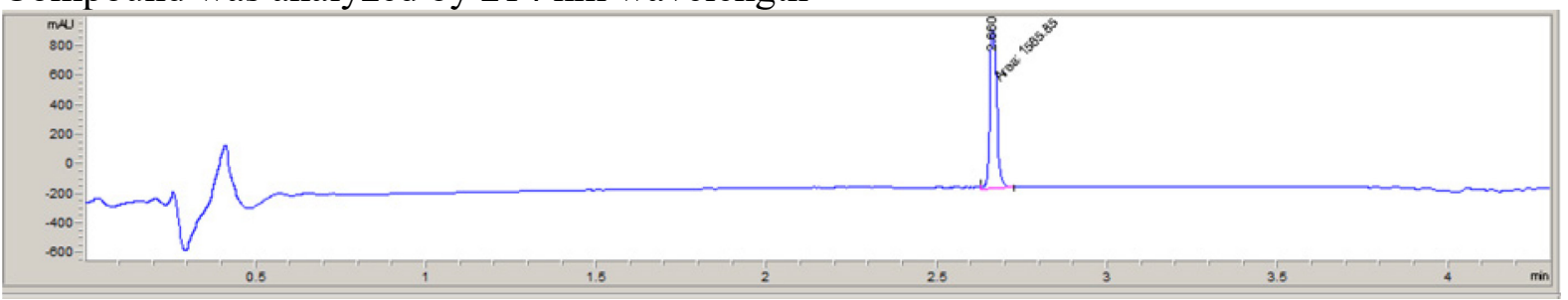




\section{Ac-Trp(Boc)-Phe(4-iodo)-Thr(OtBu)-NH2 (8e)}

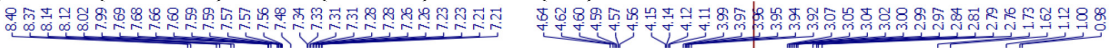

1 H NMR (300 MHz, d6-DMSO)
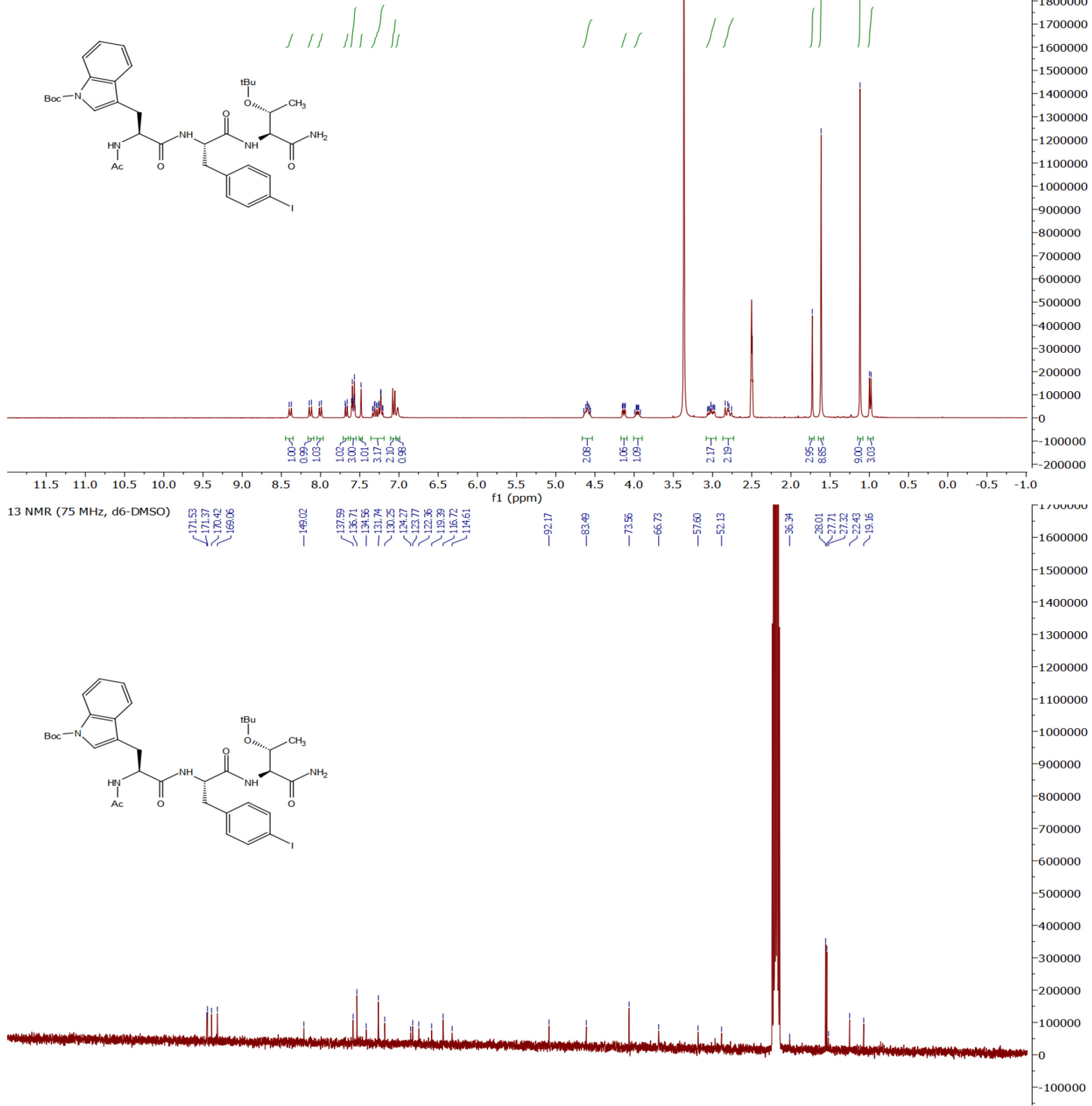

$\begin{array}{lllllllllllll}210 & 200 & 190 & 180 & 170 & 160 & 150 & 140 & 130 & 120 & 110 & 100 & 90\end{array}$

Compound was analyzed by $214 \mathrm{~nm}$ wavelength

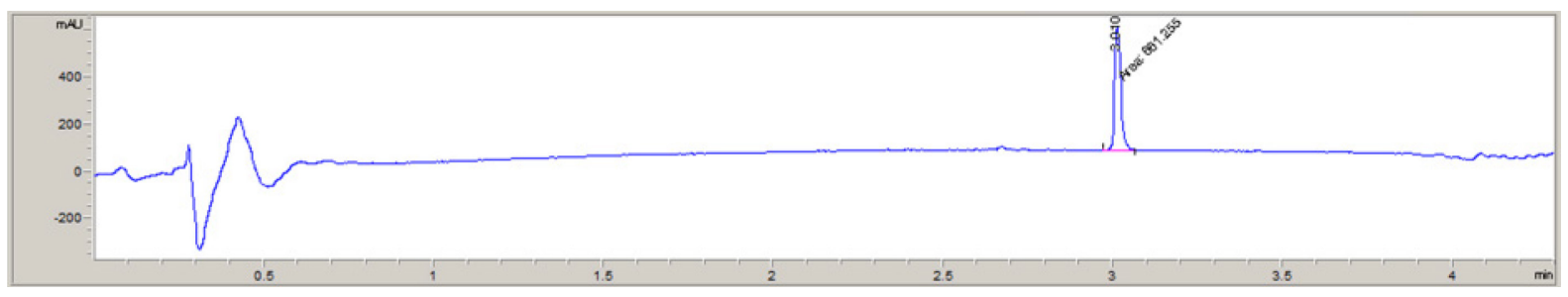




\section{$\operatorname{Ac}-\operatorname{Trp}(\mathrm{Boc})-p \mathrm{CMF}(\mathrm{O} t \mathrm{Bu})-\mathrm{Thr}(\mathrm{O} t \mathrm{Bu})-\mathrm{NH}_{2}(9 \mathrm{e})$}
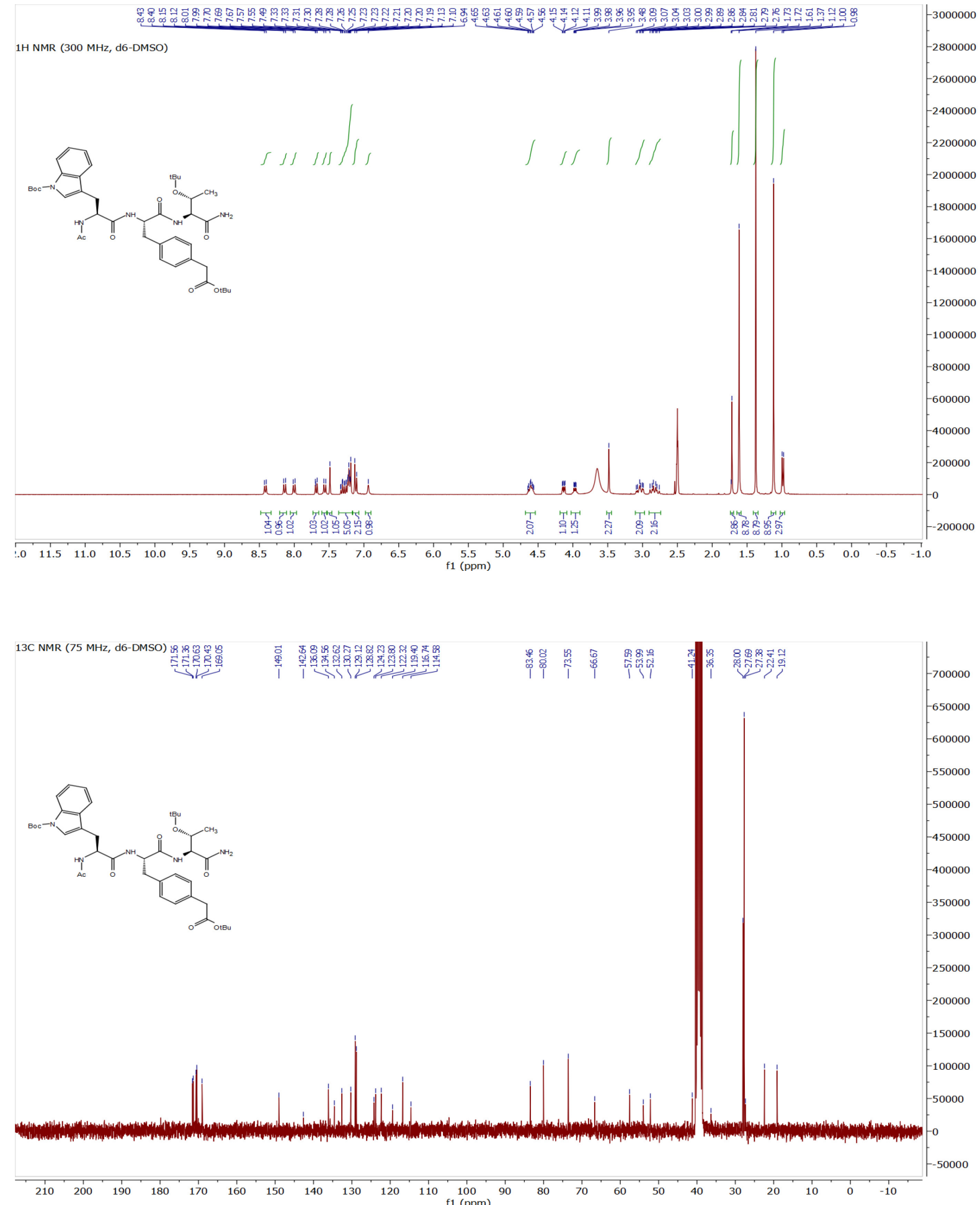

Compound was analyzed by $214 \mathrm{~nm}$ wavelength

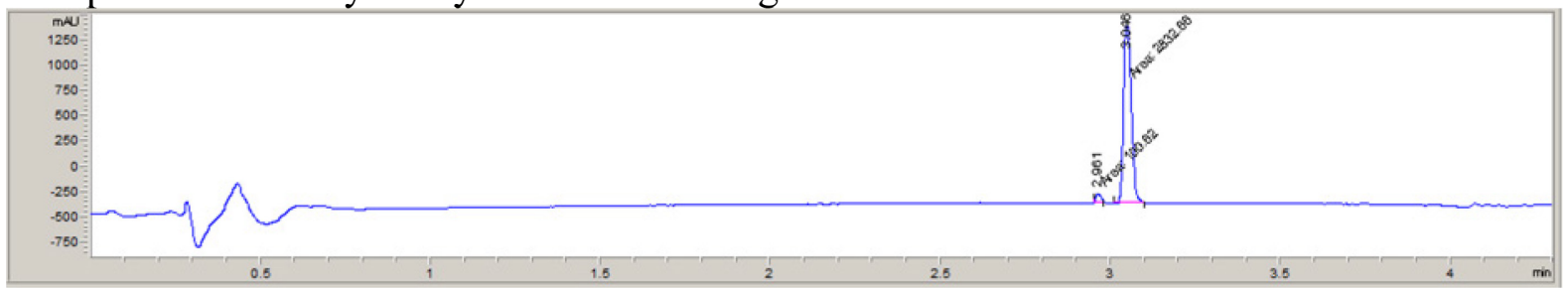




\section{$\operatorname{Ac}-\operatorname{Arg}(\mathrm{Pbf})-\mathrm{Phe}(4-i o d o)-\mathrm{Thr}(\mathrm{O} t \mathrm{Bu})-\mathrm{NH}_{2}(8 f)$}

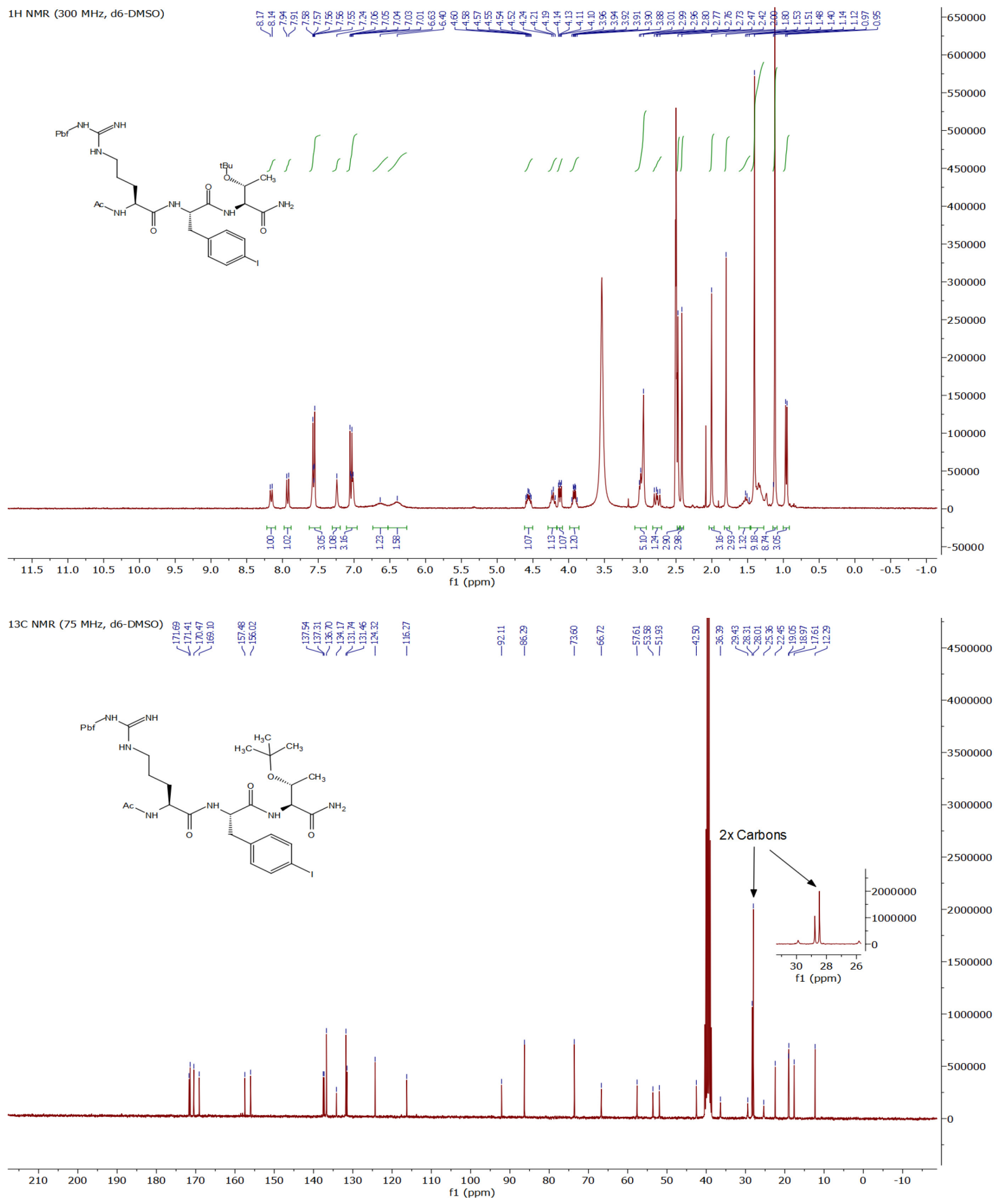




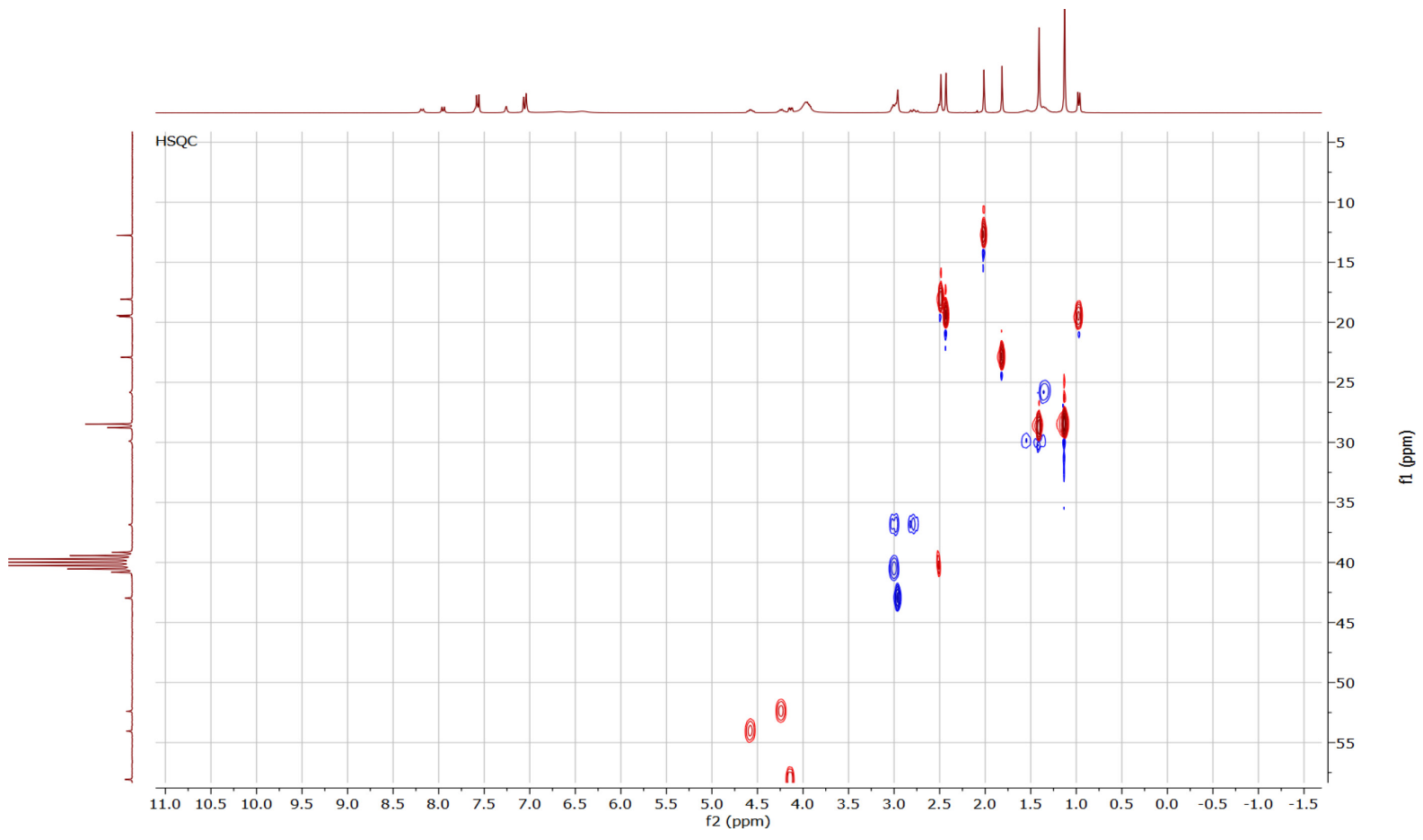

Compound was analyzed by $214 \mathrm{~nm}$ wavelength

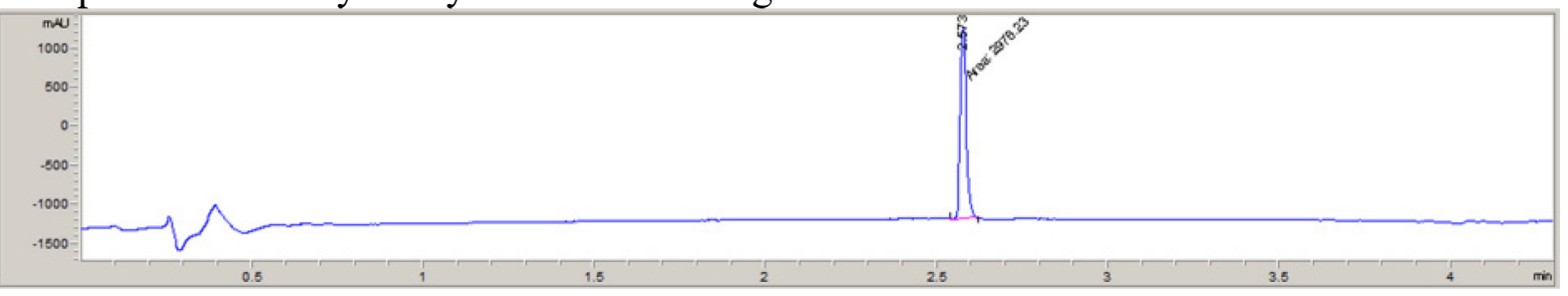




\section{$\operatorname{Ac}-\operatorname{Arg}(\mathrm{Pbf})-p \mathrm{CMF}(\mathrm{O} t \mathrm{Bu})-\mathrm{Thr}(\mathrm{O} t \mathrm{Bu})-\mathrm{NH}_{2}(9 \mathrm{f})$}
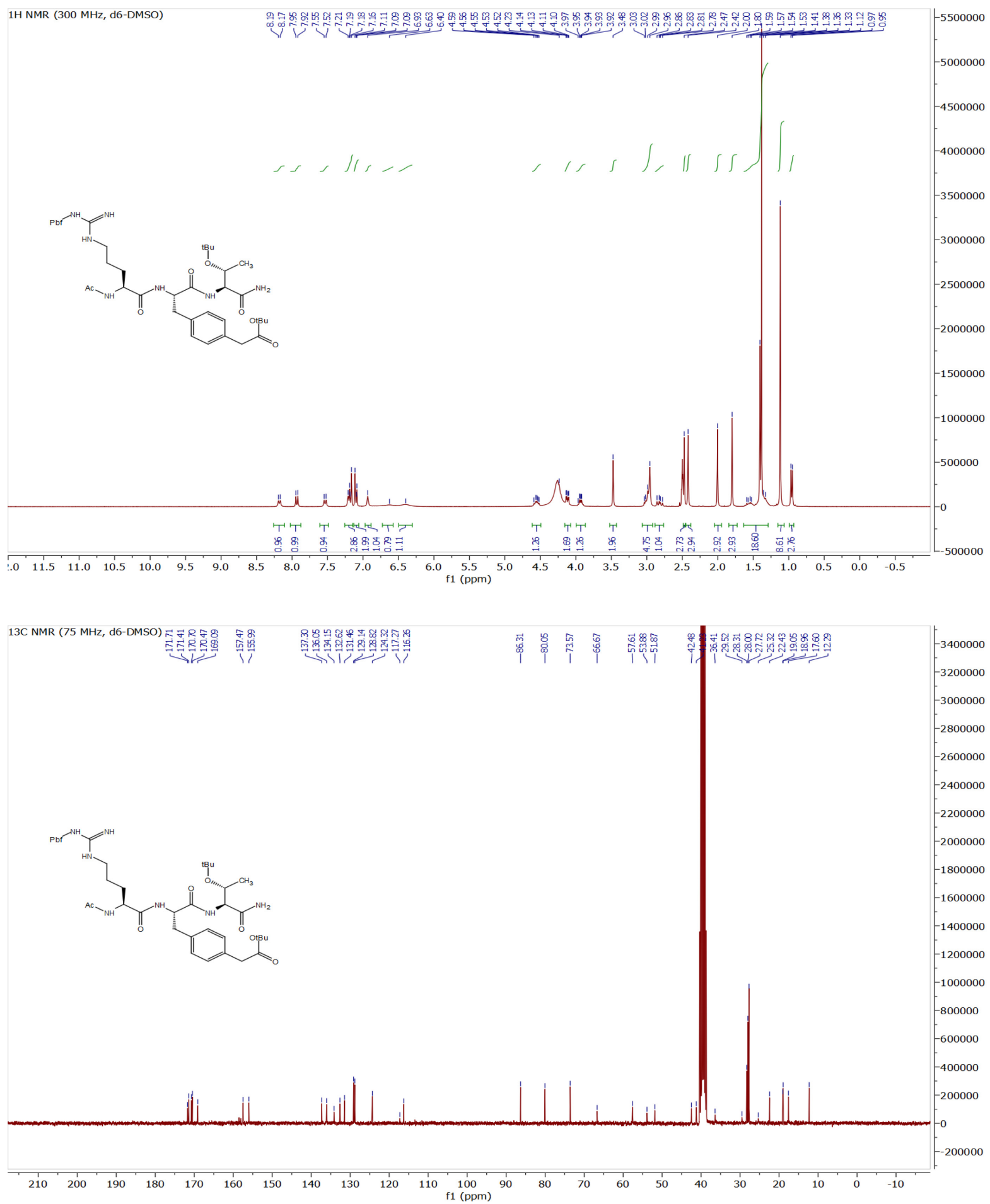

Compound was analyzed by $214 \mathrm{~nm}$ wavelength

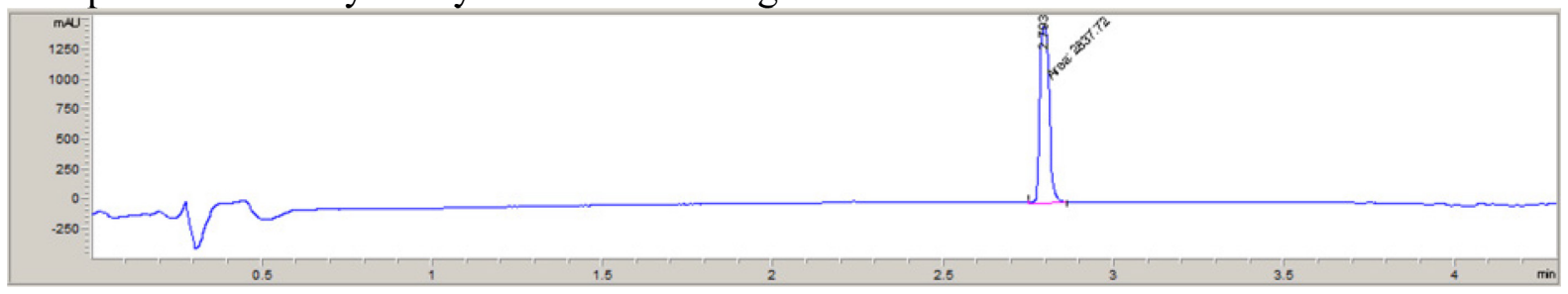




\section{Ac-Glu(OtBu)-Phe(4-Bromo)-Thr(OtBu)-NH2 (8g)}

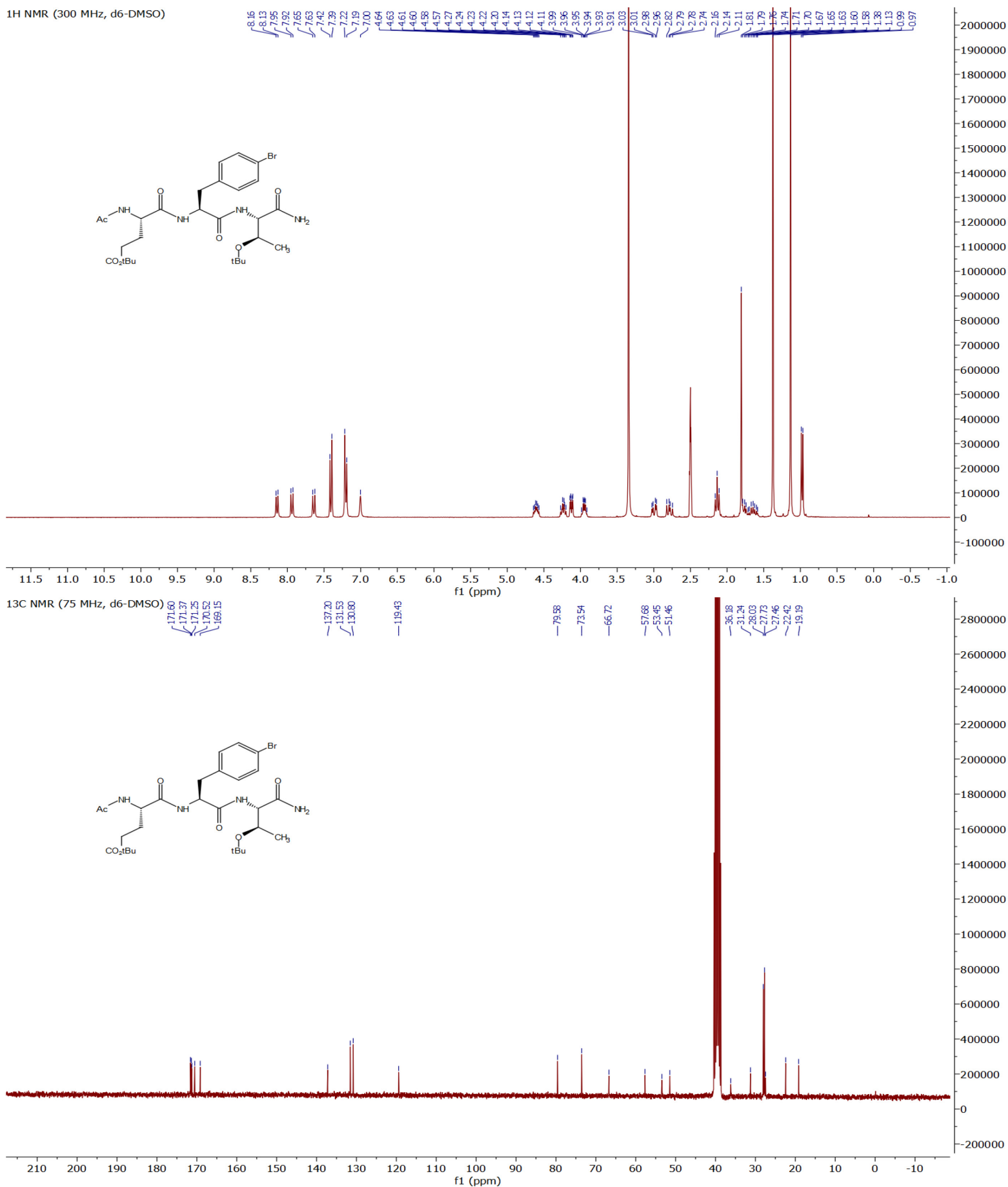

Compound was analyzed by $214 \mathrm{~nm}$ wavelength

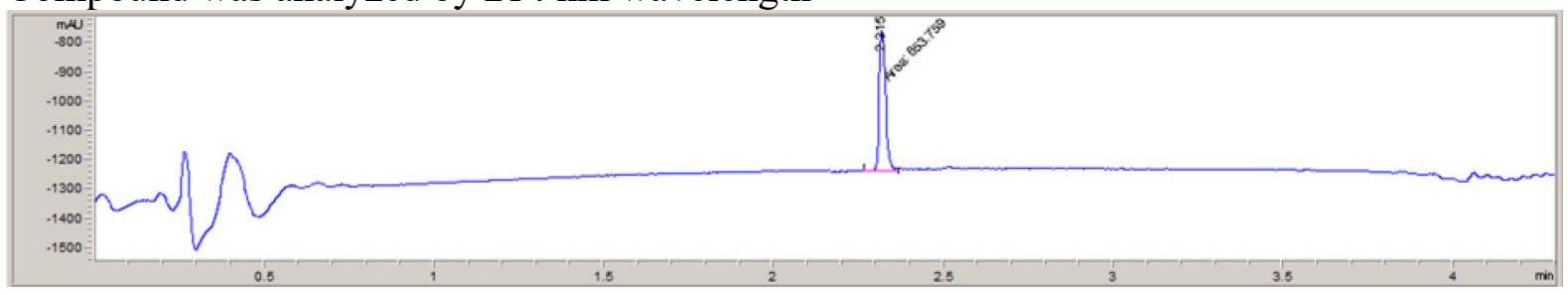




\section{Ac-Glu(OtBu)-Pmp(OEt 2$)-T h r(O t B u)(9 g)$}

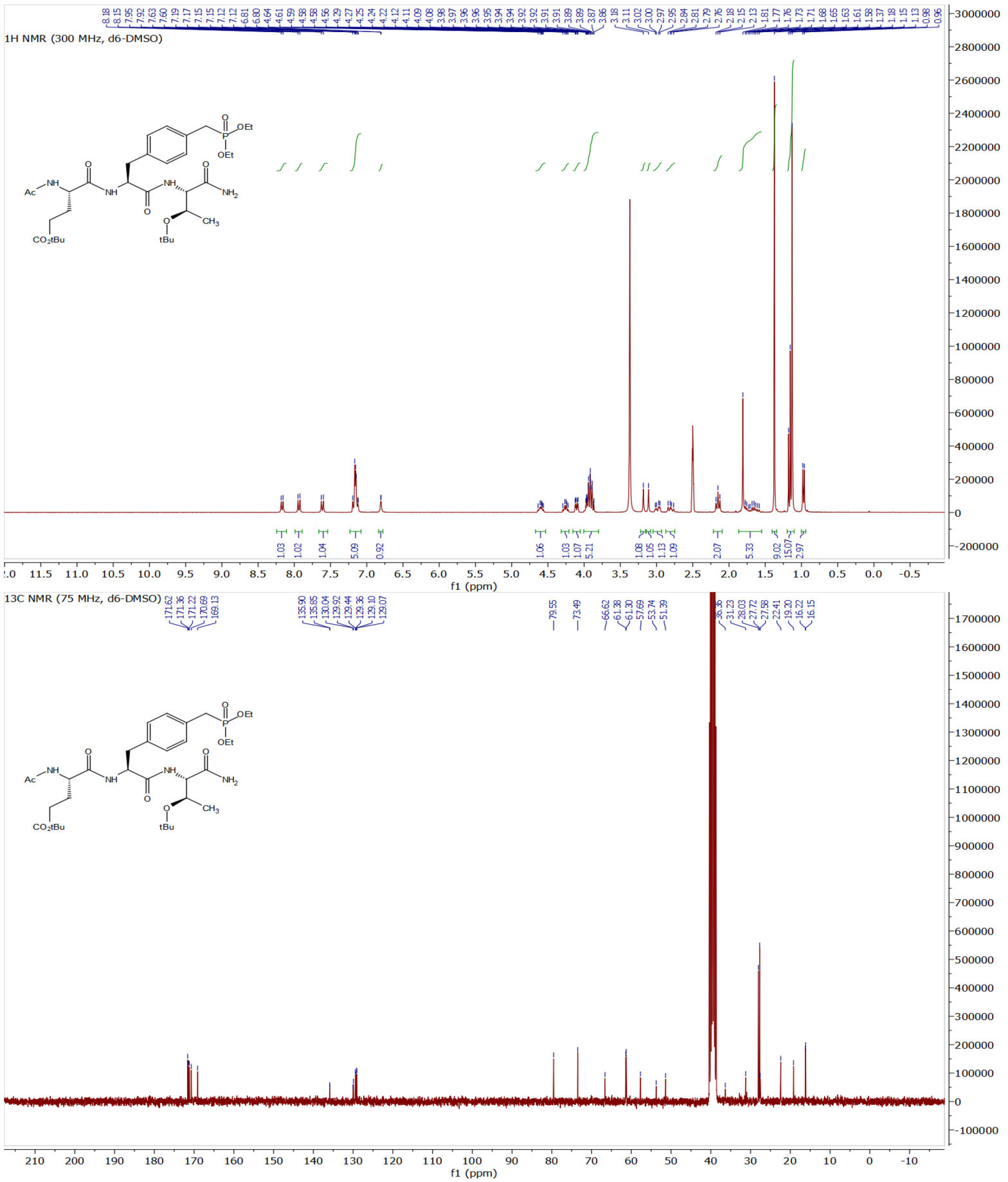




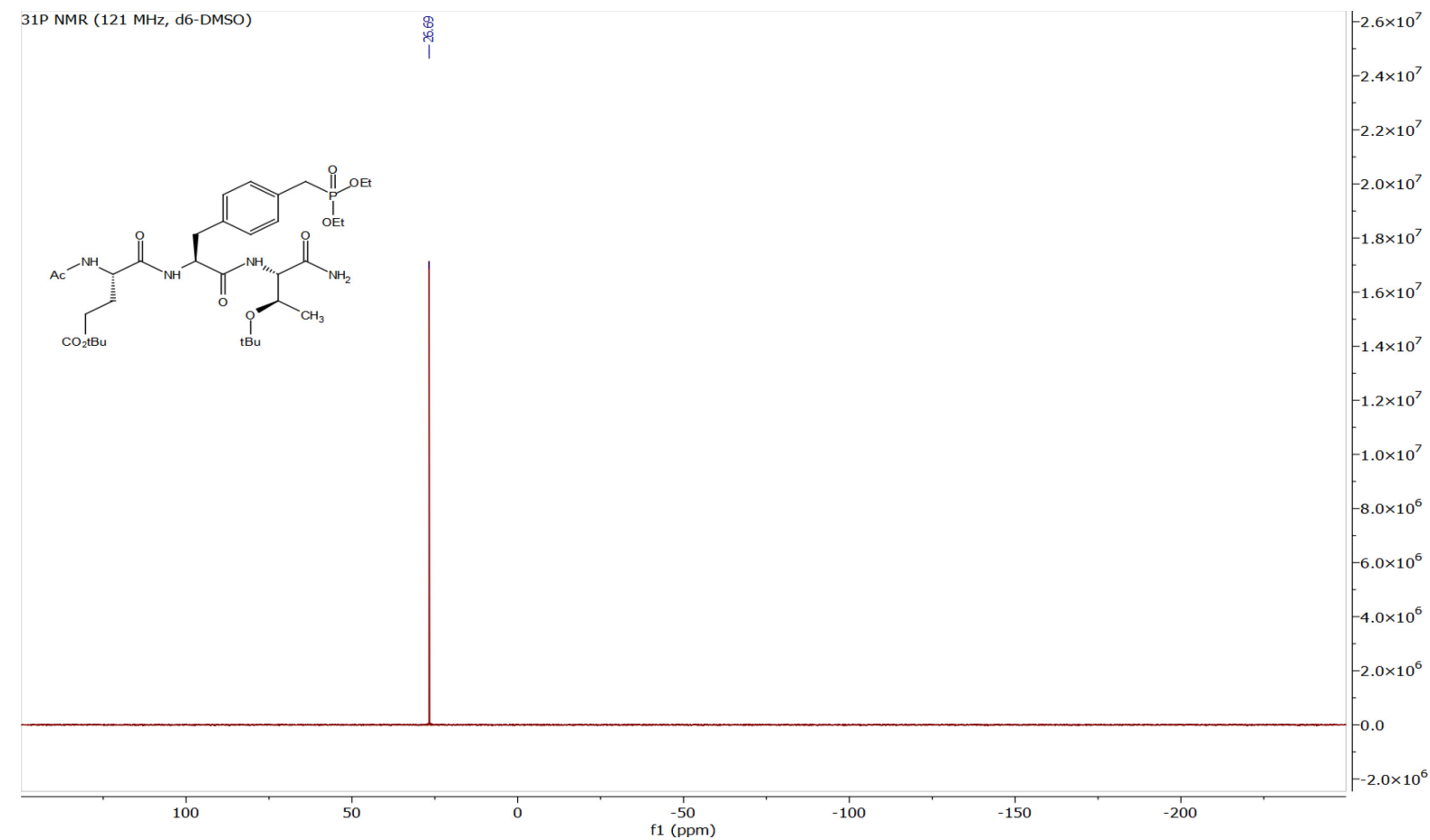

Compound was analyzed by $214 \mathrm{~nm}$ wavelength

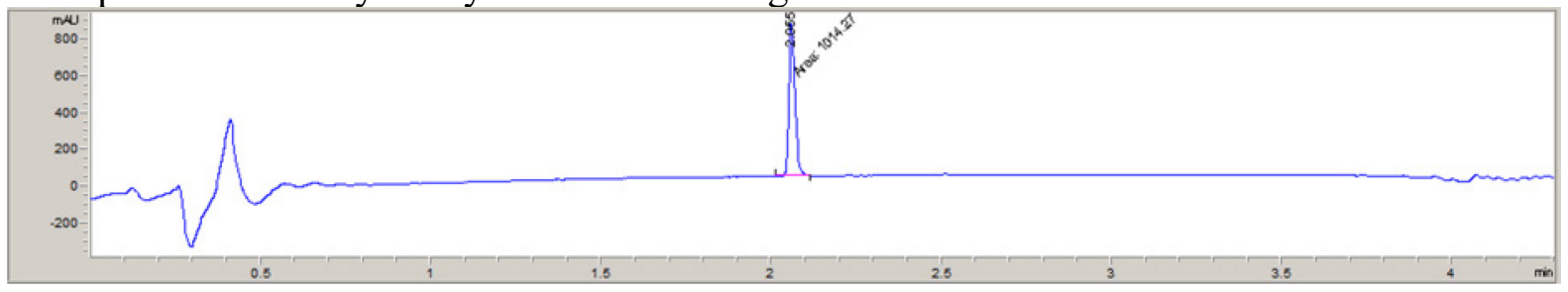




\section{Ac-Glu-Phe(4-iodo)-Thr-NH2 (10a)}
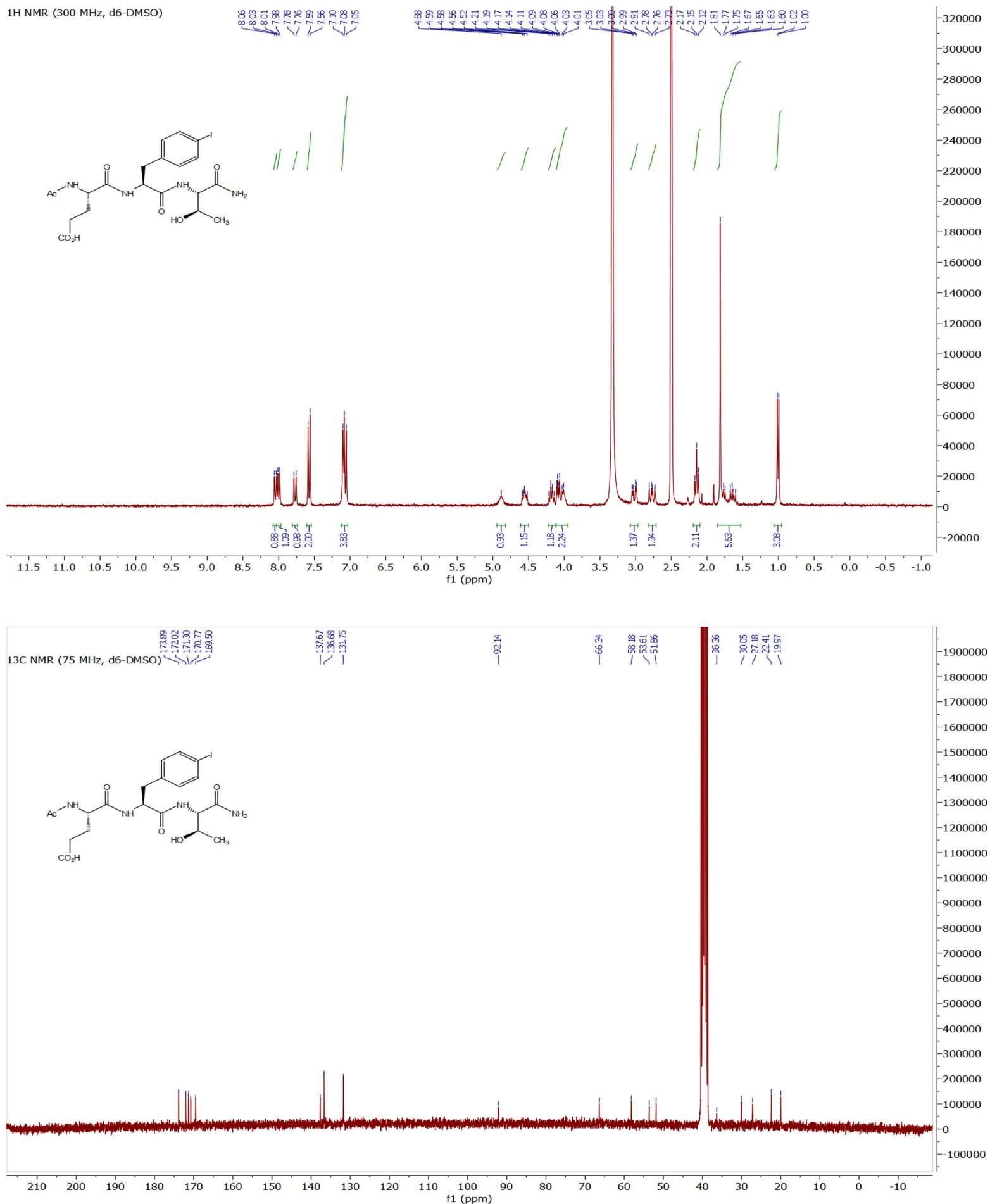

Compound was analyzed by $214 \mathrm{~nm}$ wavelength

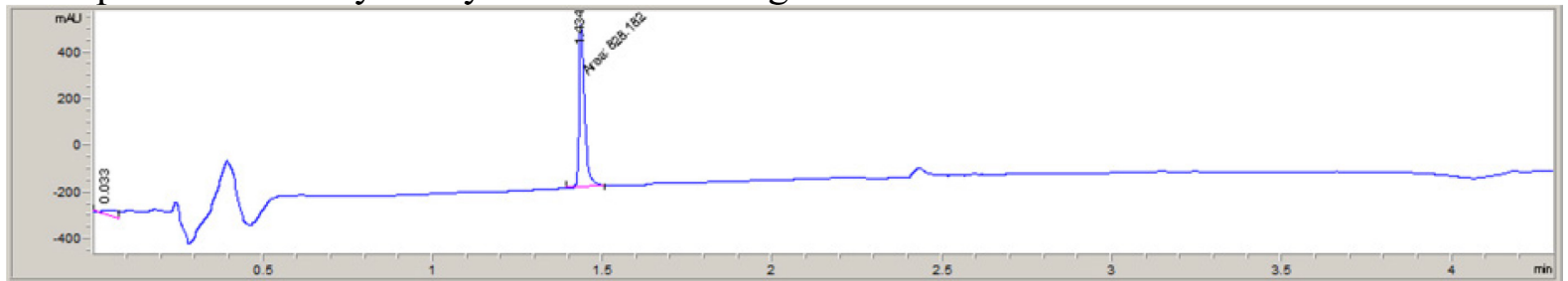




\section{Ac-Glu- $p$ CMF(OtBu)-Thr-NH2 (11a)}

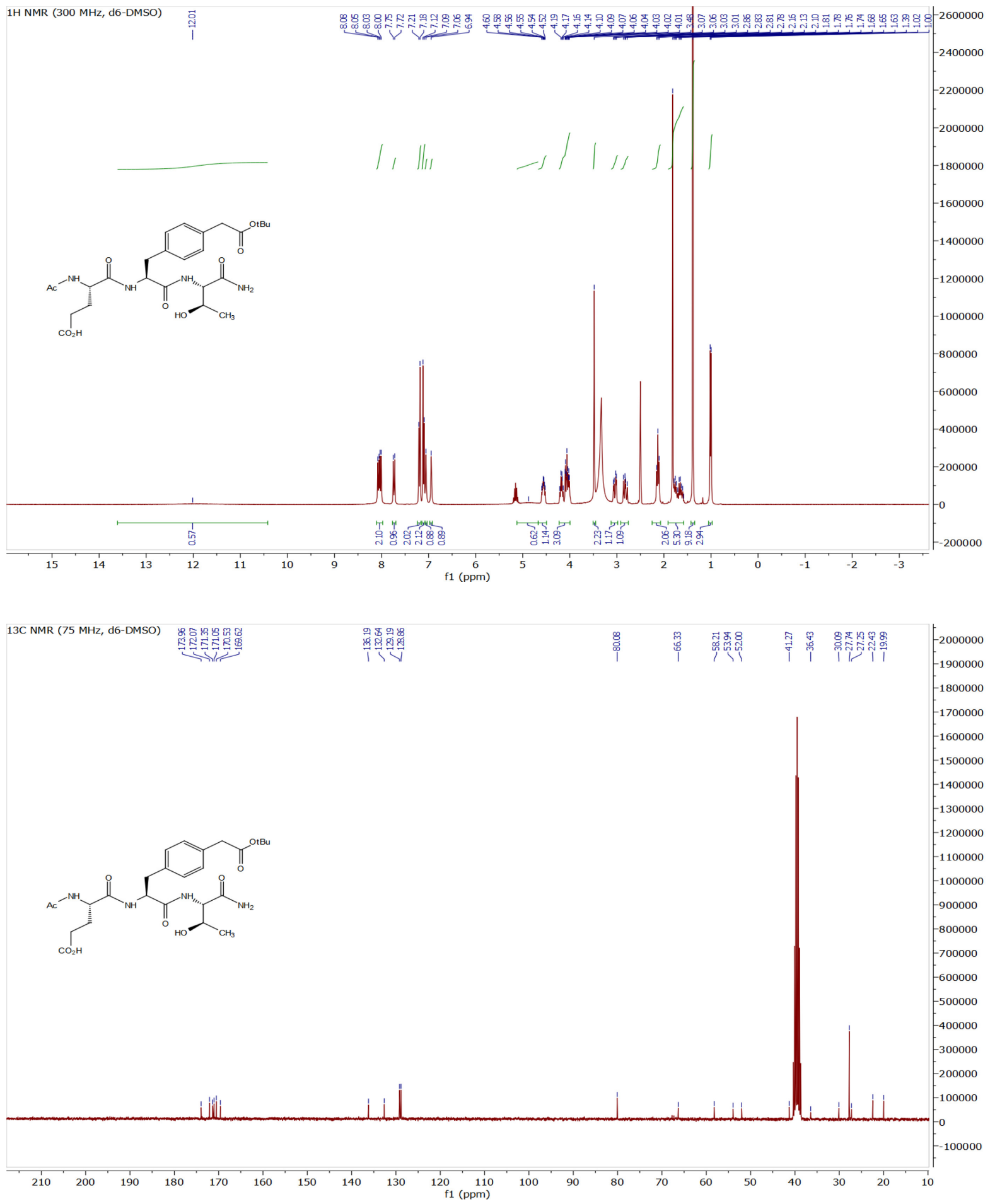

Compound was analyzed by $214 \mathrm{~nm}$ wavelength

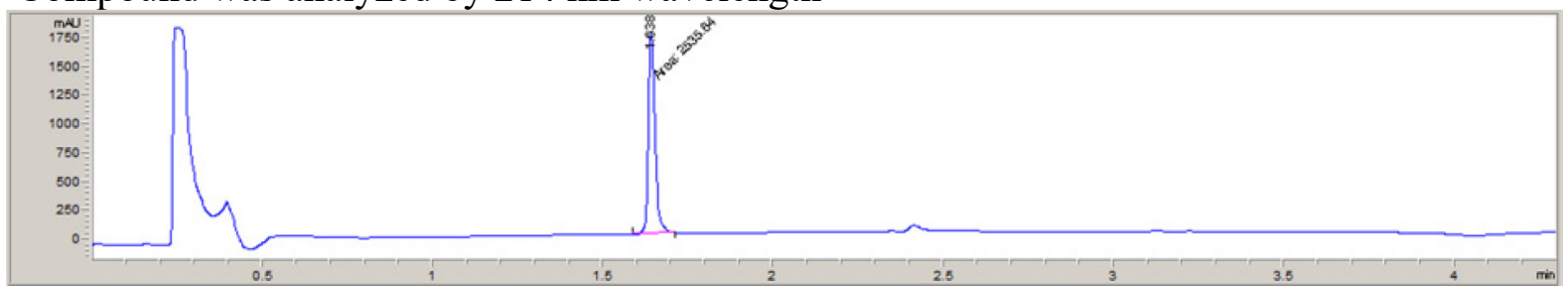




\section{Ac-Trp-Phe(4-iodo)-Thr-NH2 (10b)}

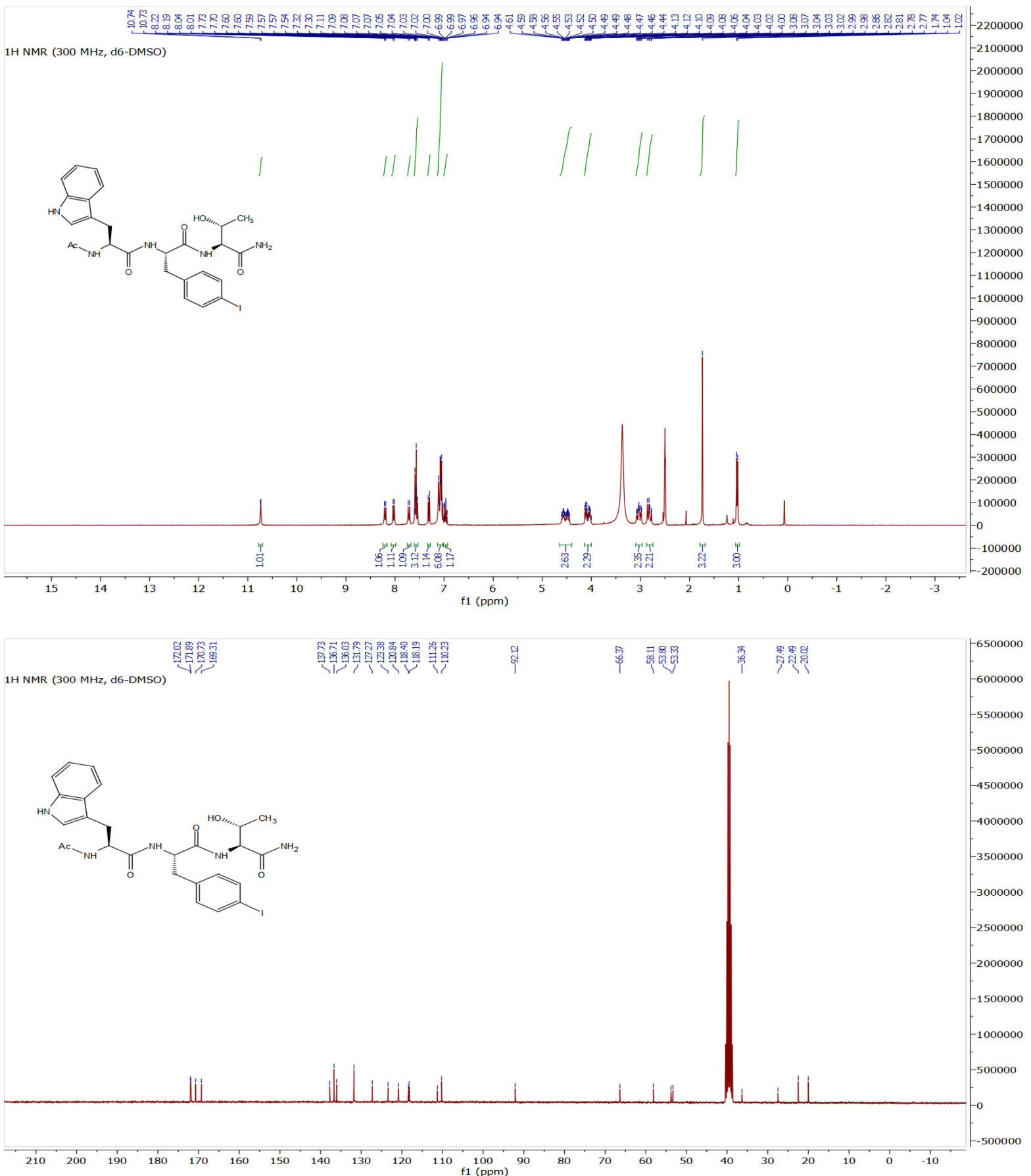

Compound was analyzed by $214 \mathrm{~nm}$ wavelength

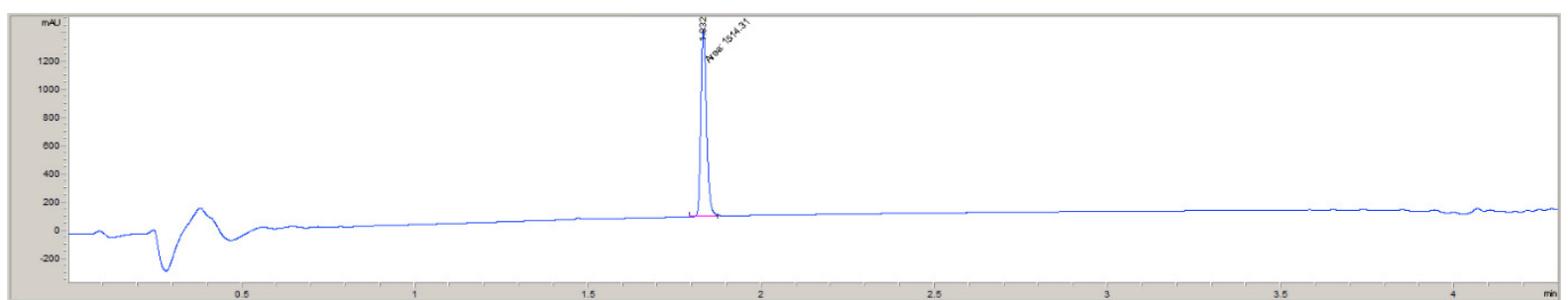




\section{Ac-Trp- $p \mathrm{CMF}(\mathrm{O} t \mathrm{Bu})-\mathrm{Thr}-\mathrm{NH}_{2}$ (11b)}
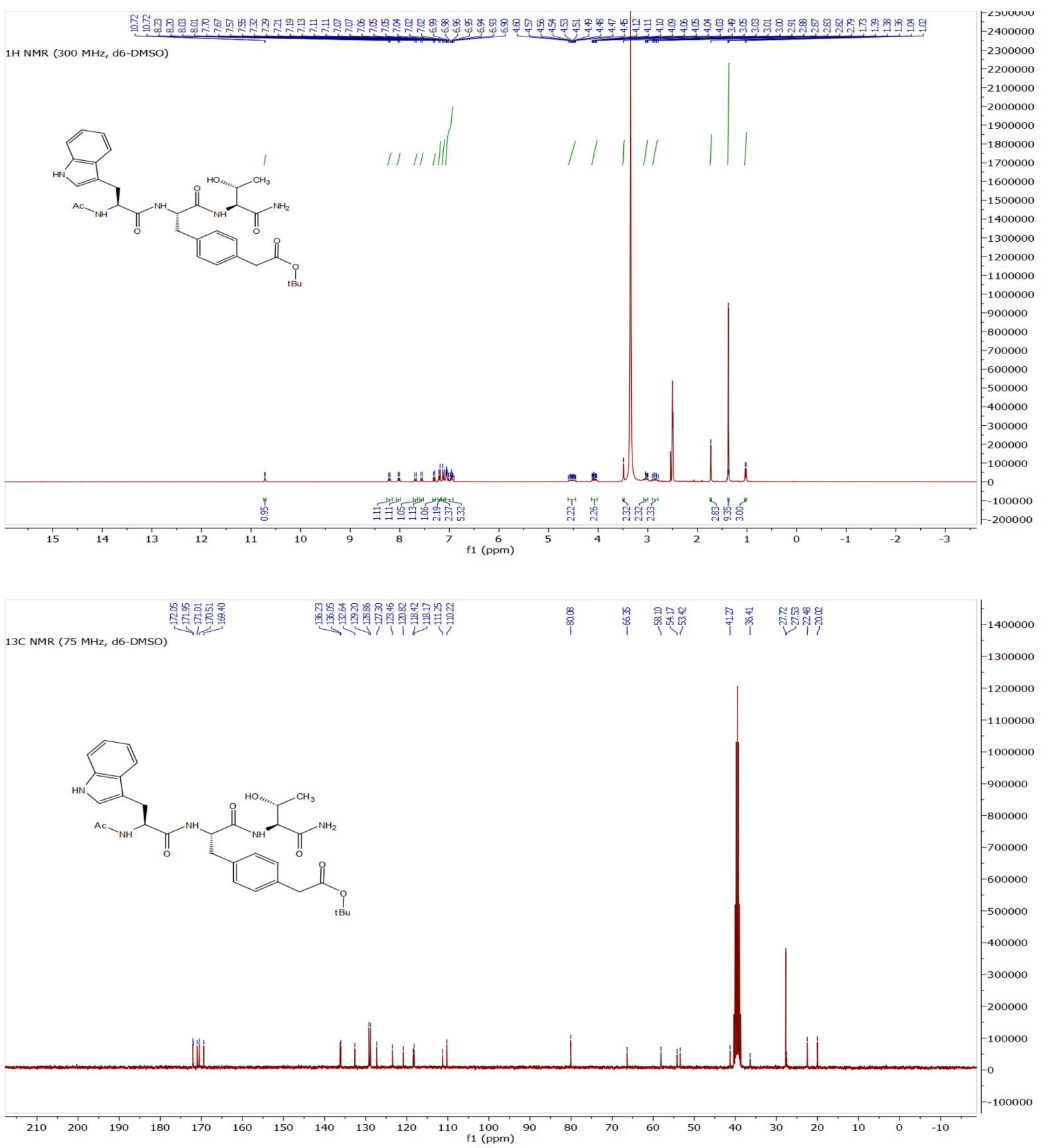


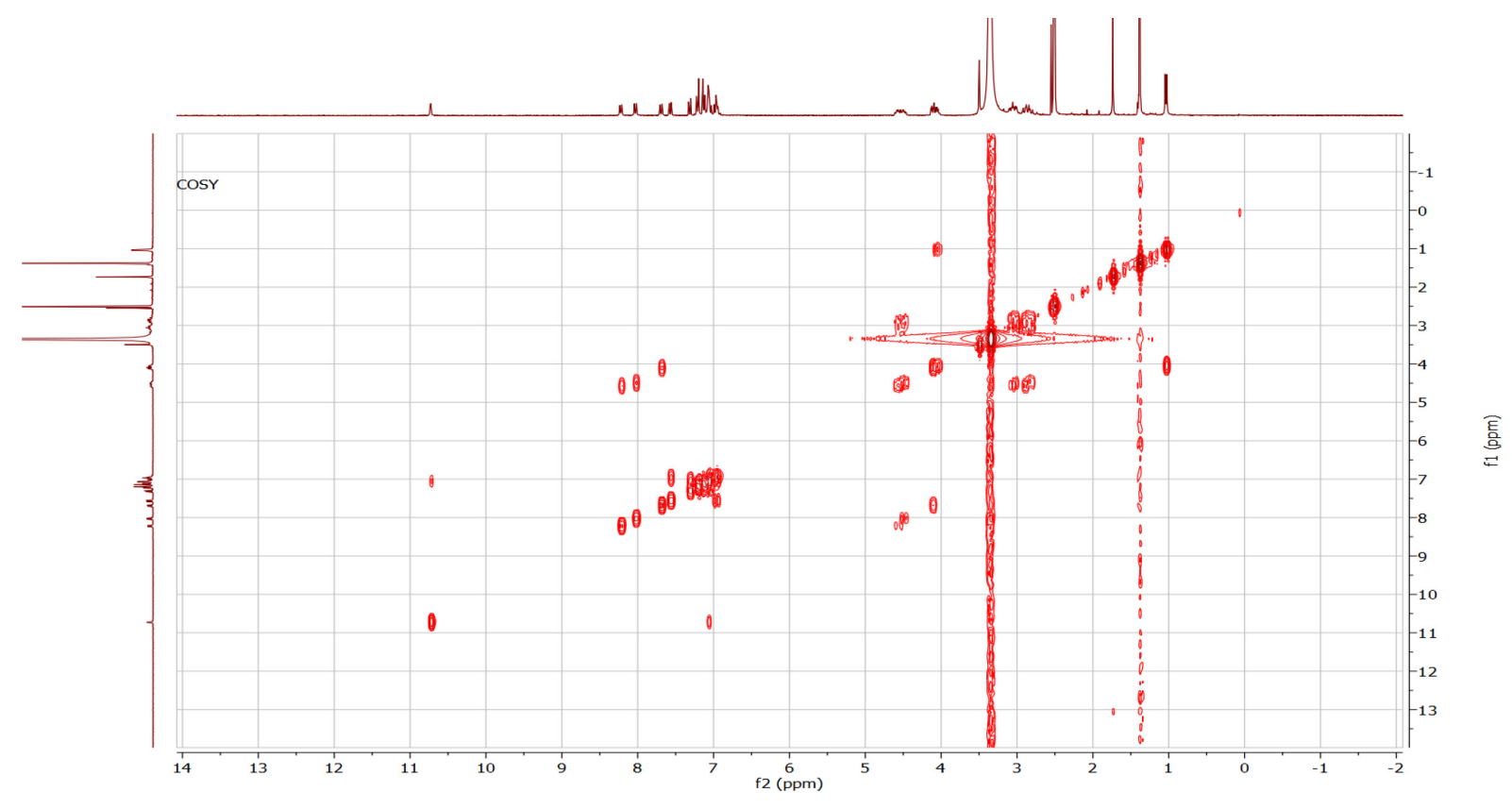

Compound was analyzed by $214 \mathrm{~nm}$ wavelength

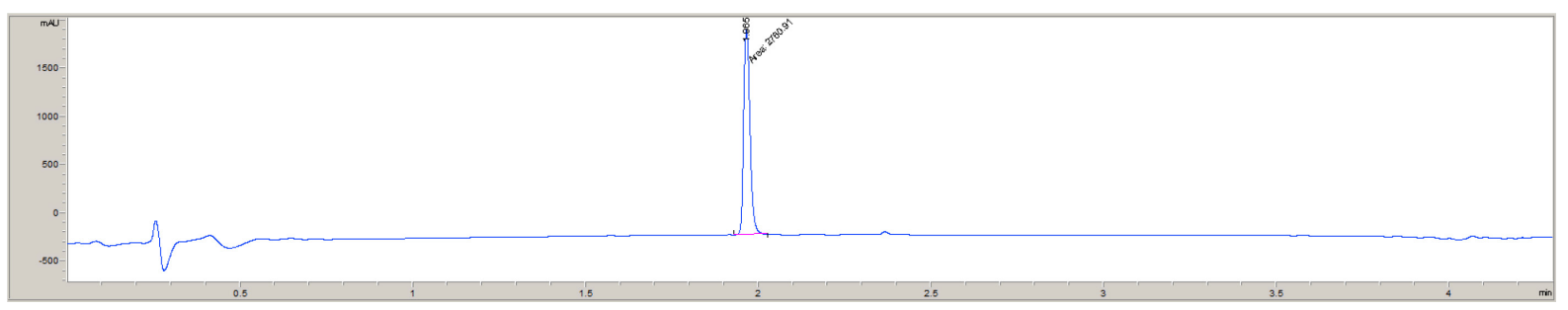




\section{Ac-Glu-Phe(4-iodo)-Ser-Thr-Val-NH 2 (10c)}
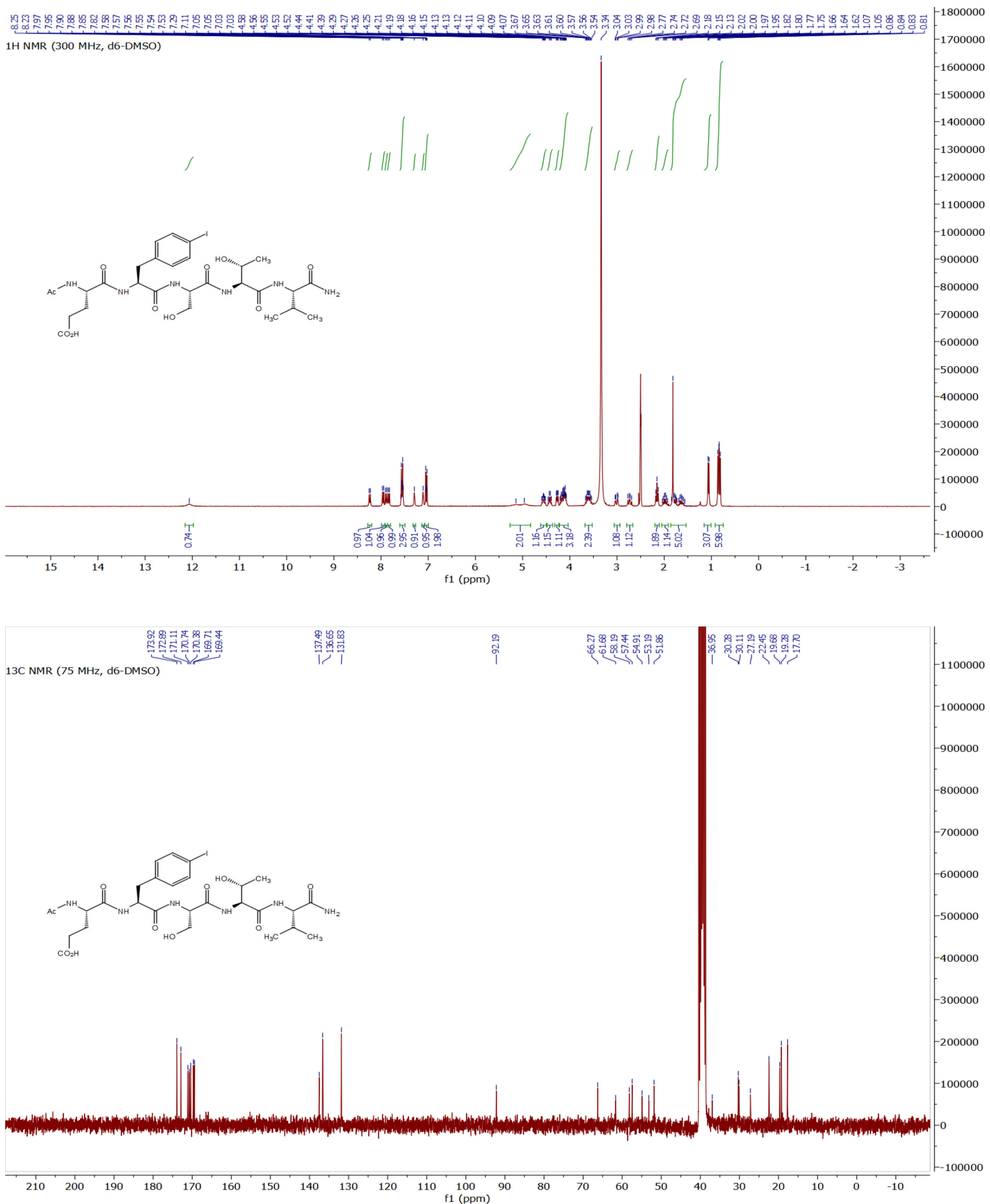

Compound was analyzed by $214 \mathrm{~nm}$ wavelength

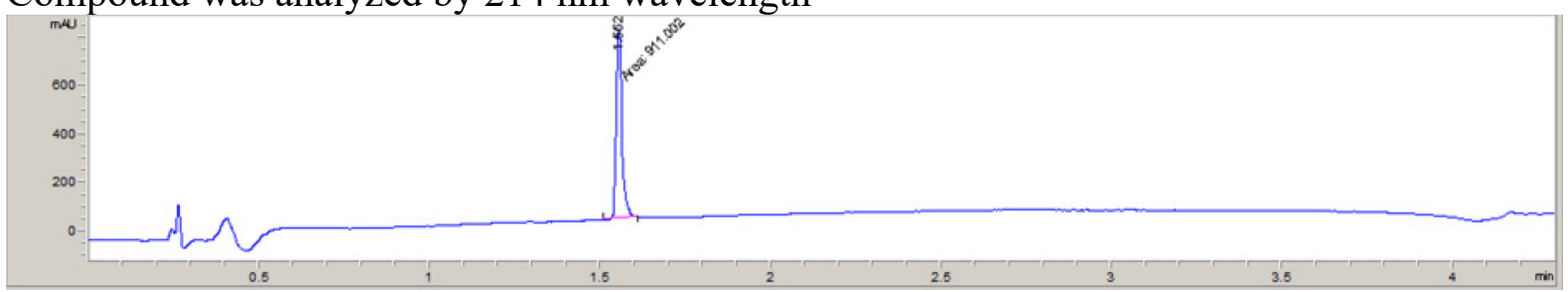




\section{Ac-Glu- $p$ CMF(OtBu)-Ser-Thr-Val-NH2 (11c)}
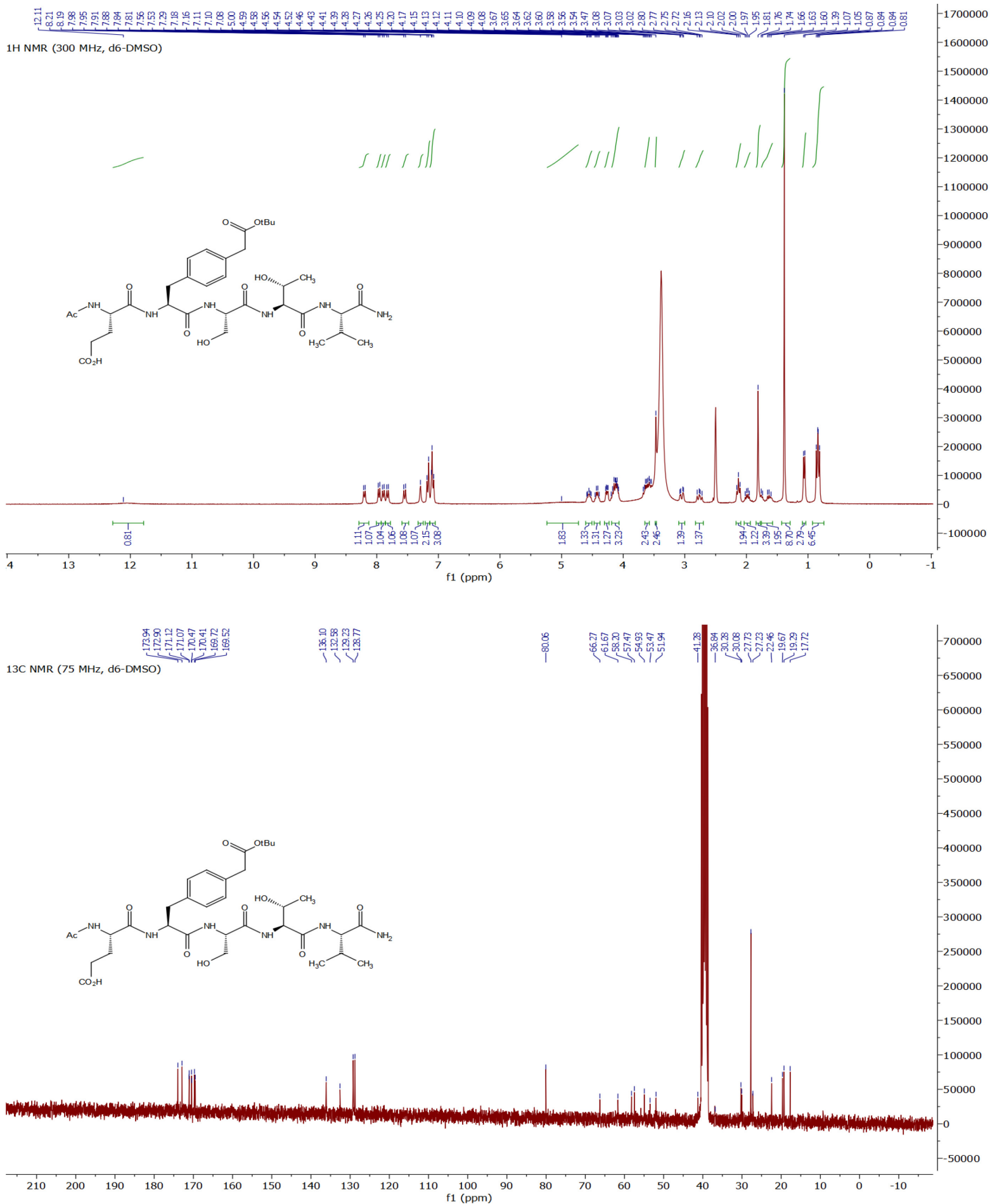

Compound was analyzed by $214 \mathrm{~nm}$ wavelength

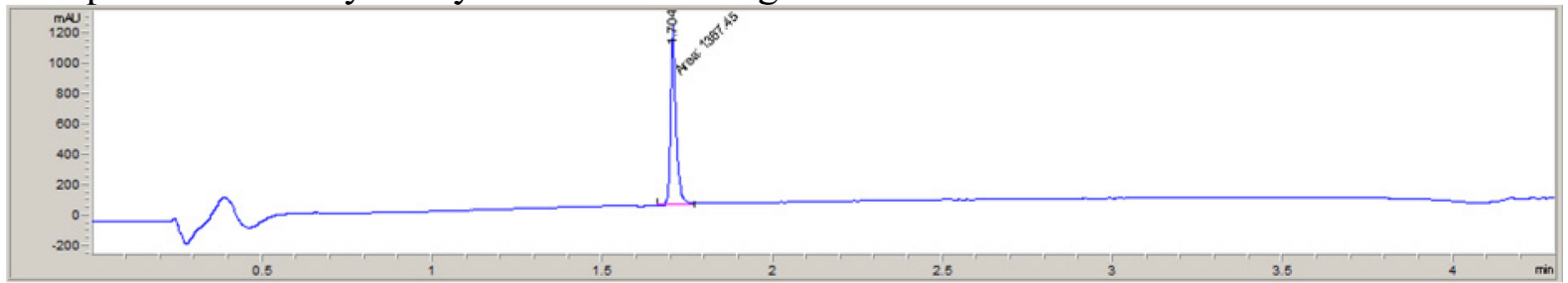




\section{Ac-Glu-Phe(4-iodo)-Tyr-Lys-Val-NH 2 (10d)}

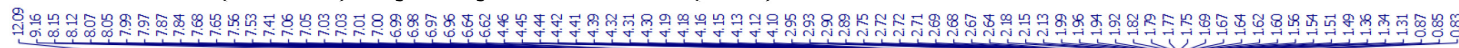

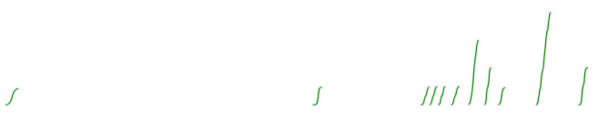

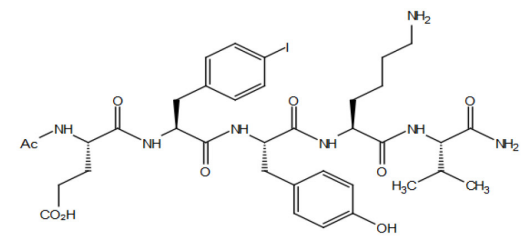
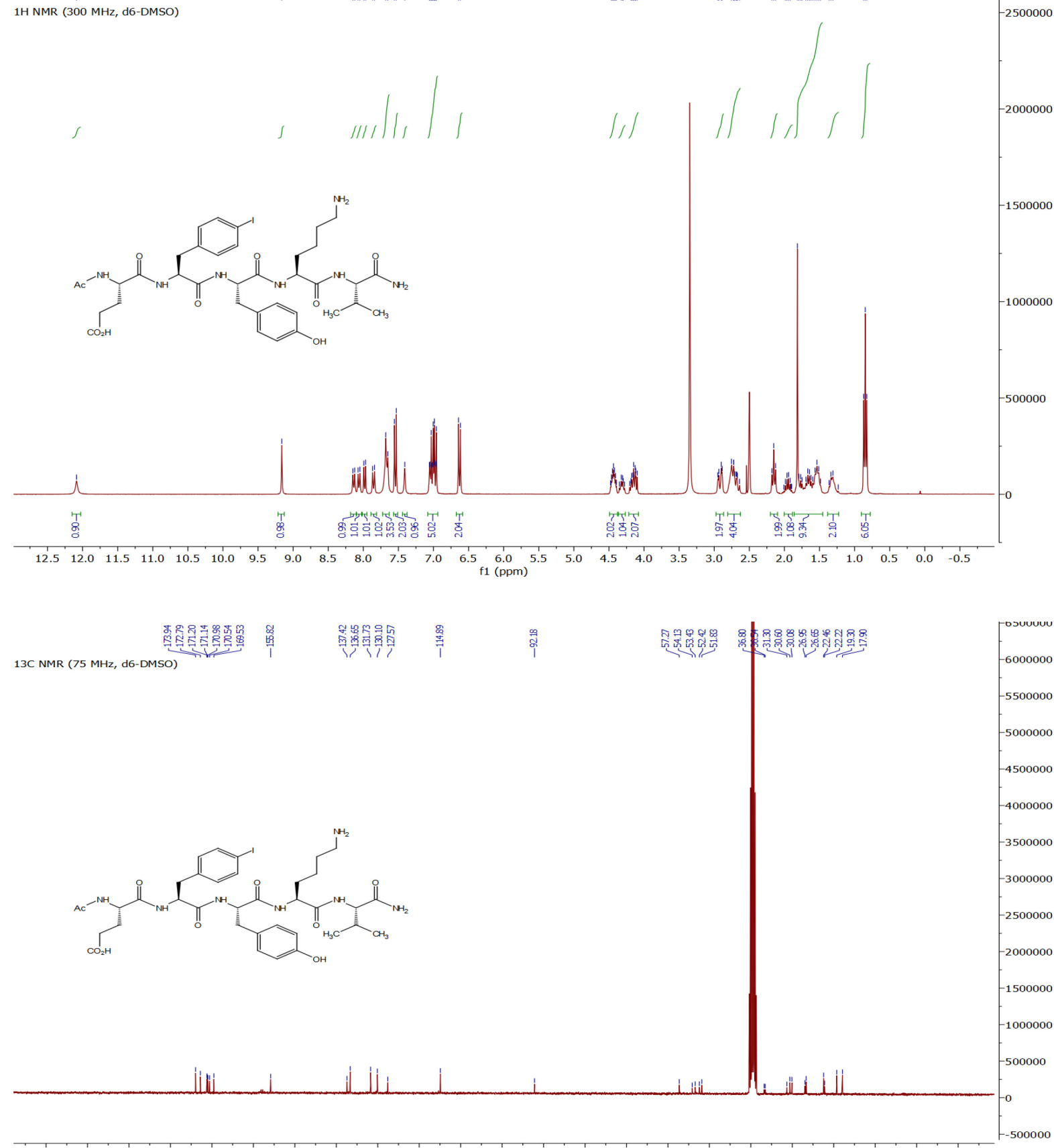

Compound was analyzed by $214 \mathrm{~nm}$ wavelength

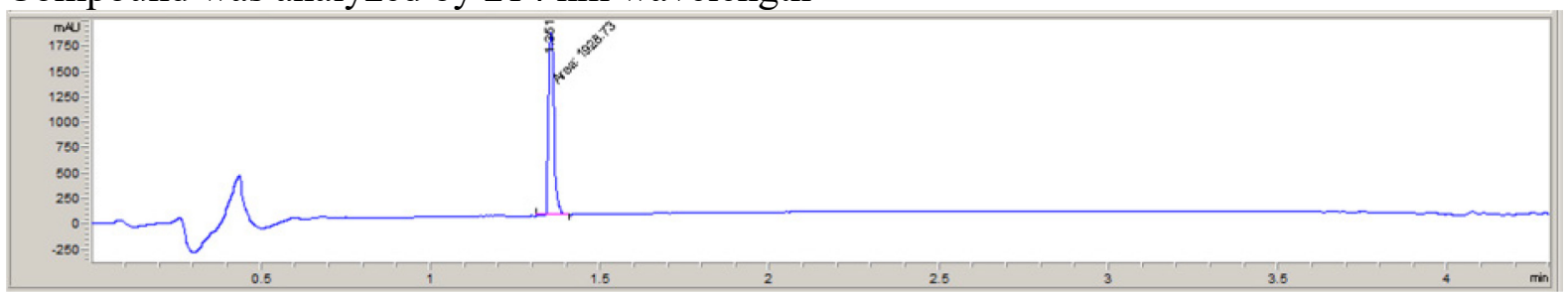


Ac-Glu- $p$ CMF(OtBu)-Tyr-Lys-Val-NH2 (11d)
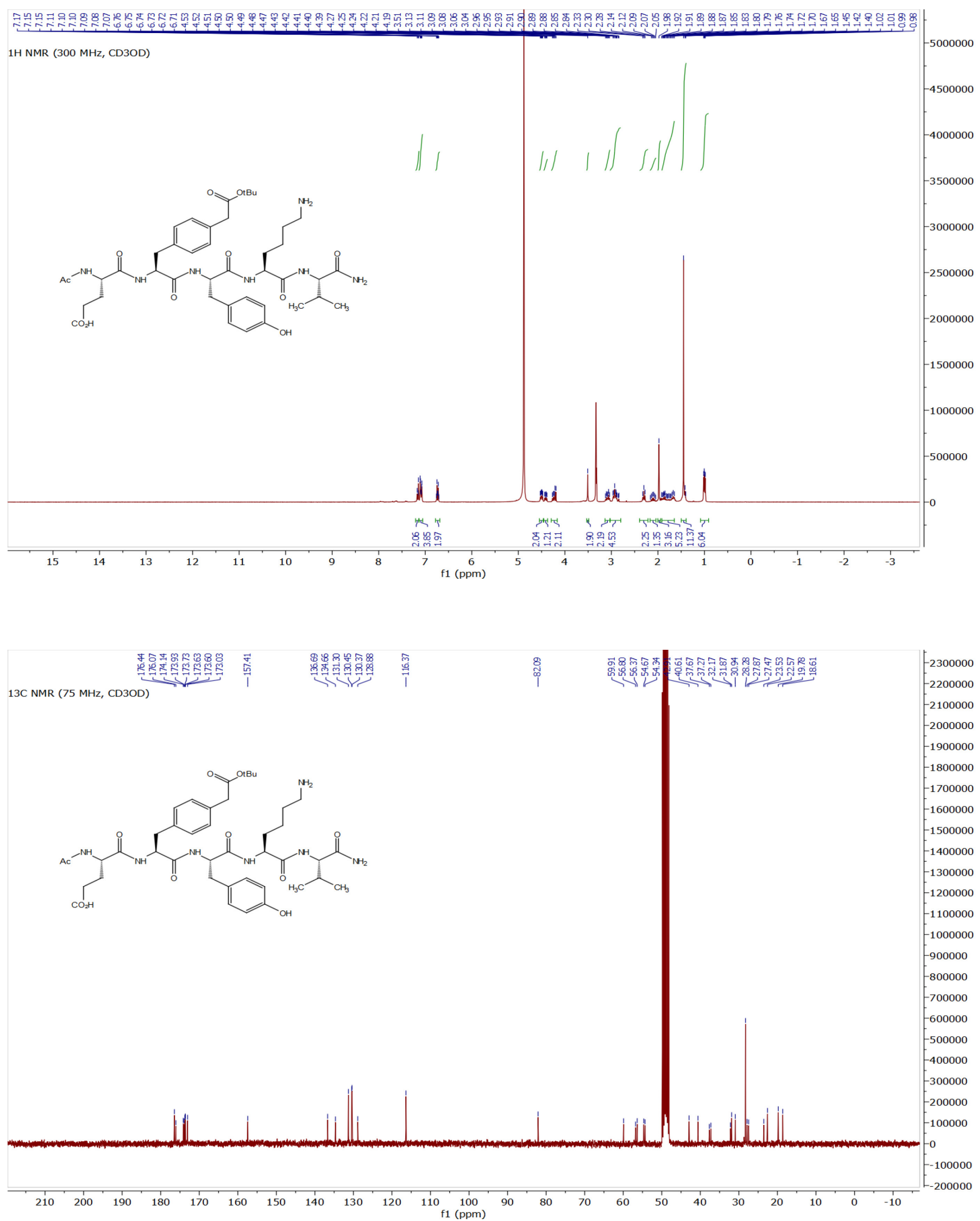


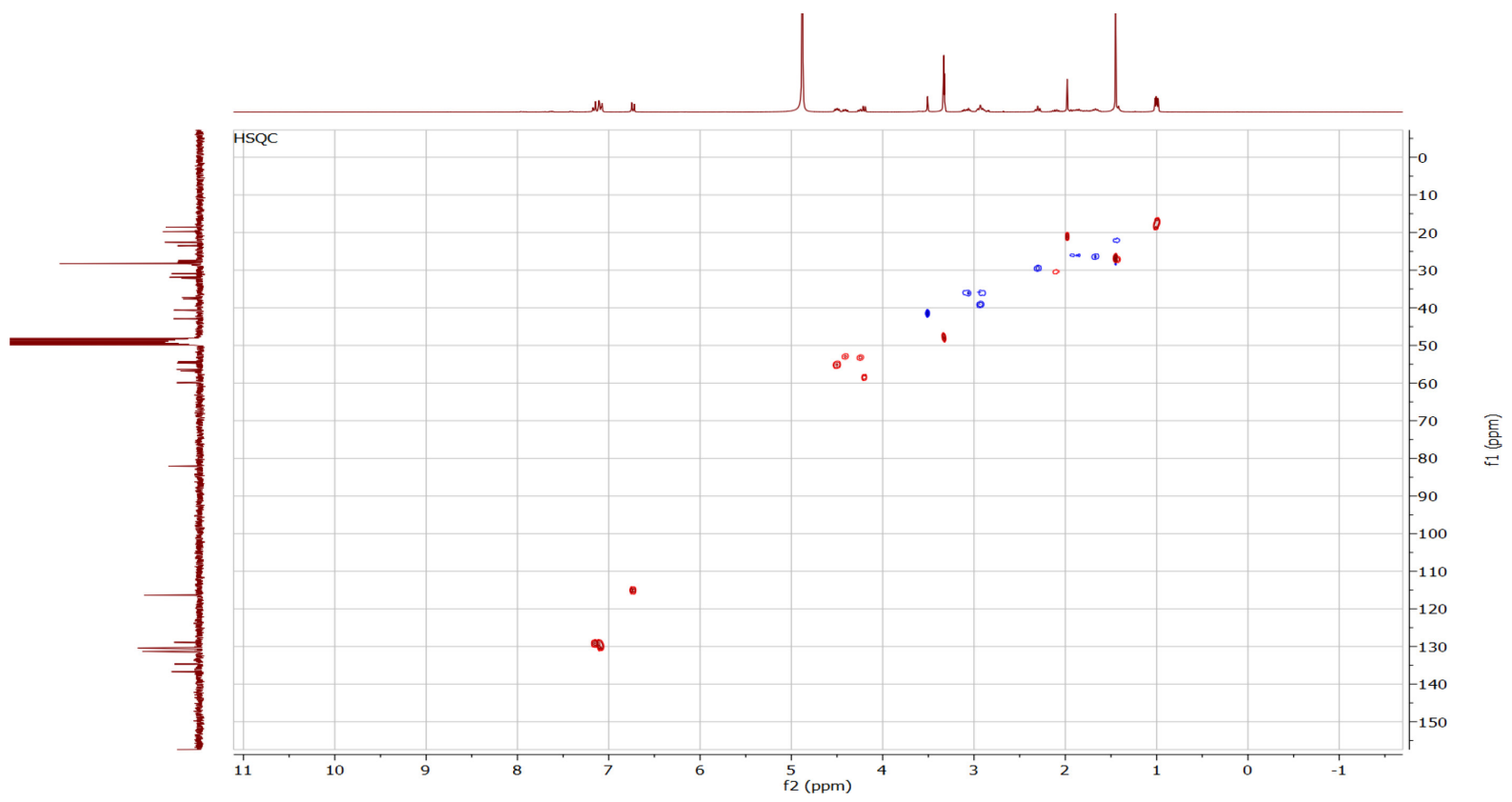

Compound was analyzed by $214 \mathrm{~nm}$ wavelength

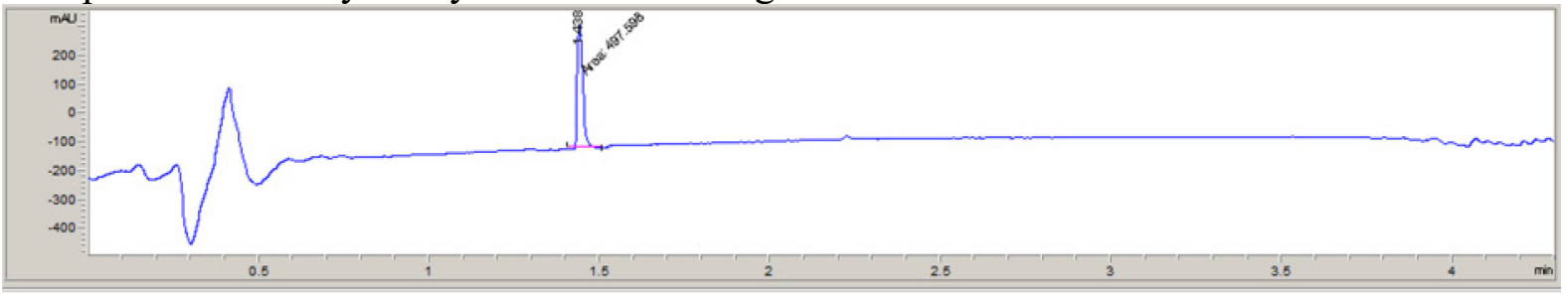




\section{Ac-Glu-Phe(4-bromo)-Ser-Thr-Val-NH2 (10e)}
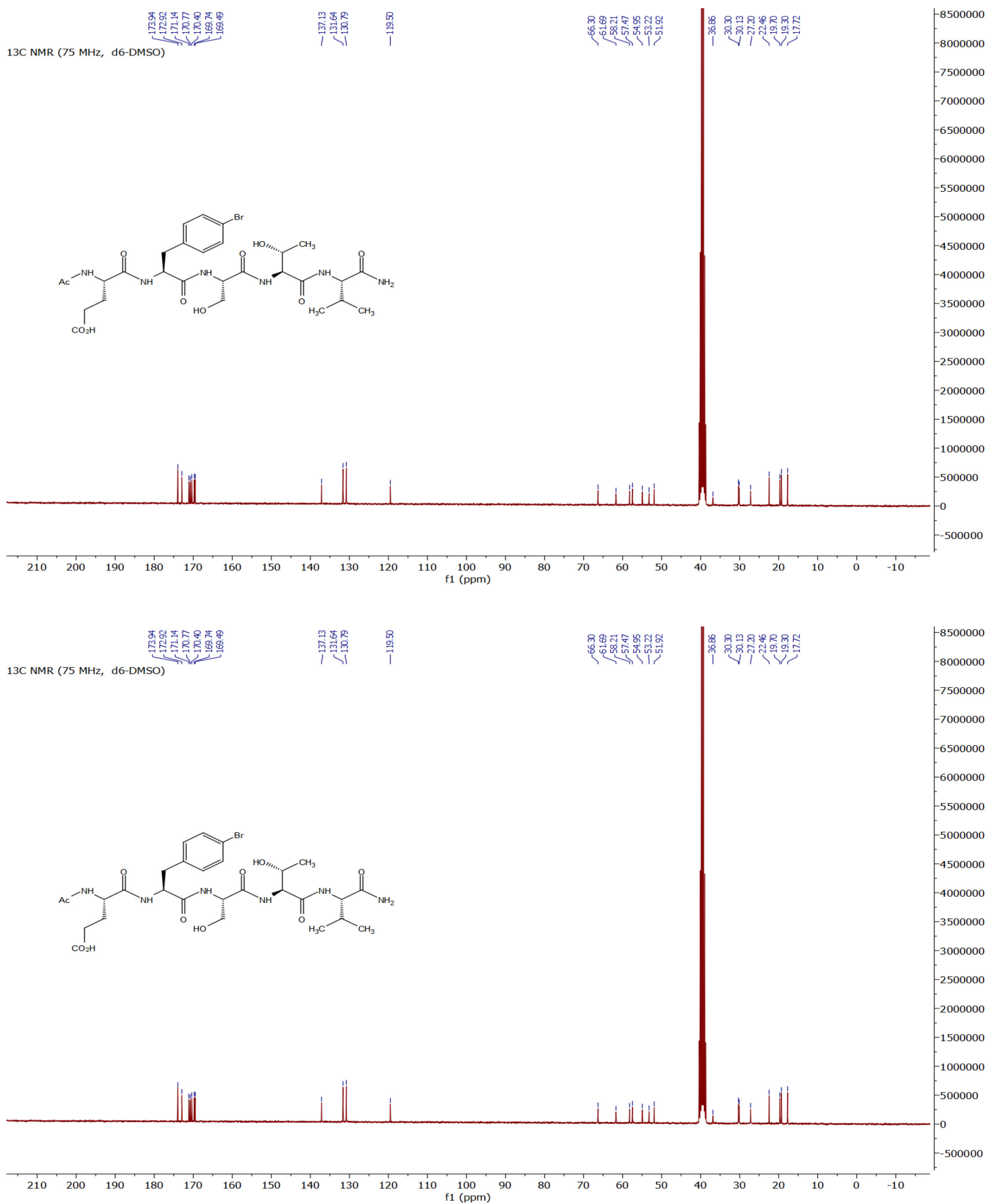

Compound was analyzed by $214 \mathrm{~nm}$ wavelength

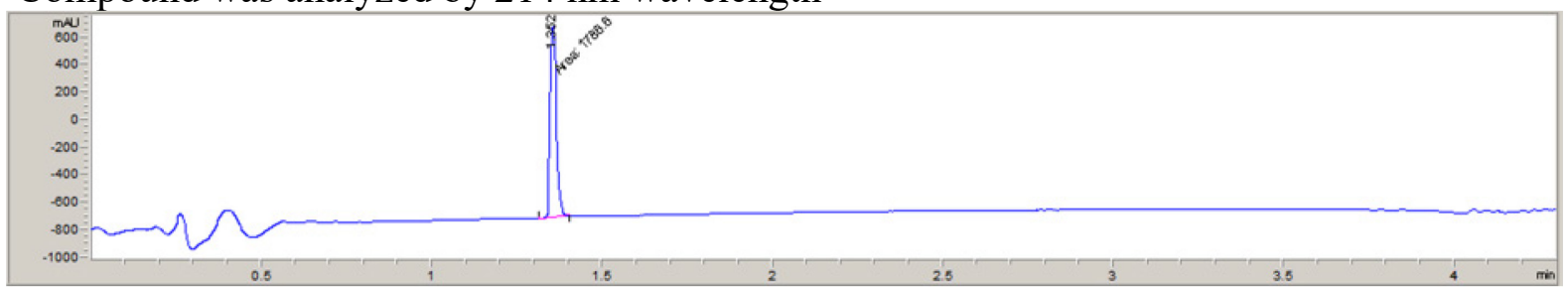




\section{Ac-Glu-Pmp(OEt) $)_{2}$-Ser-Thr-Val-NH2 (11e)}
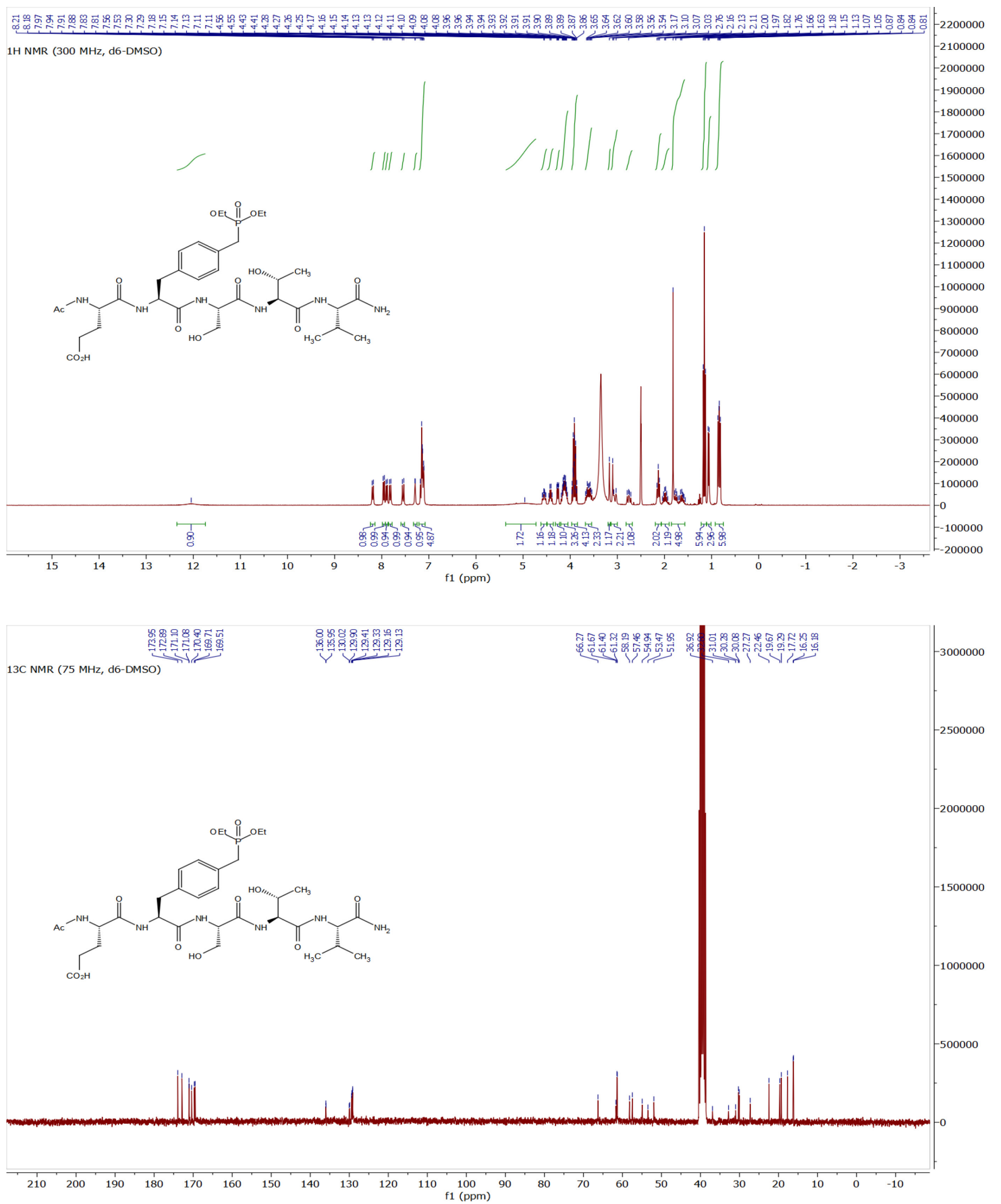


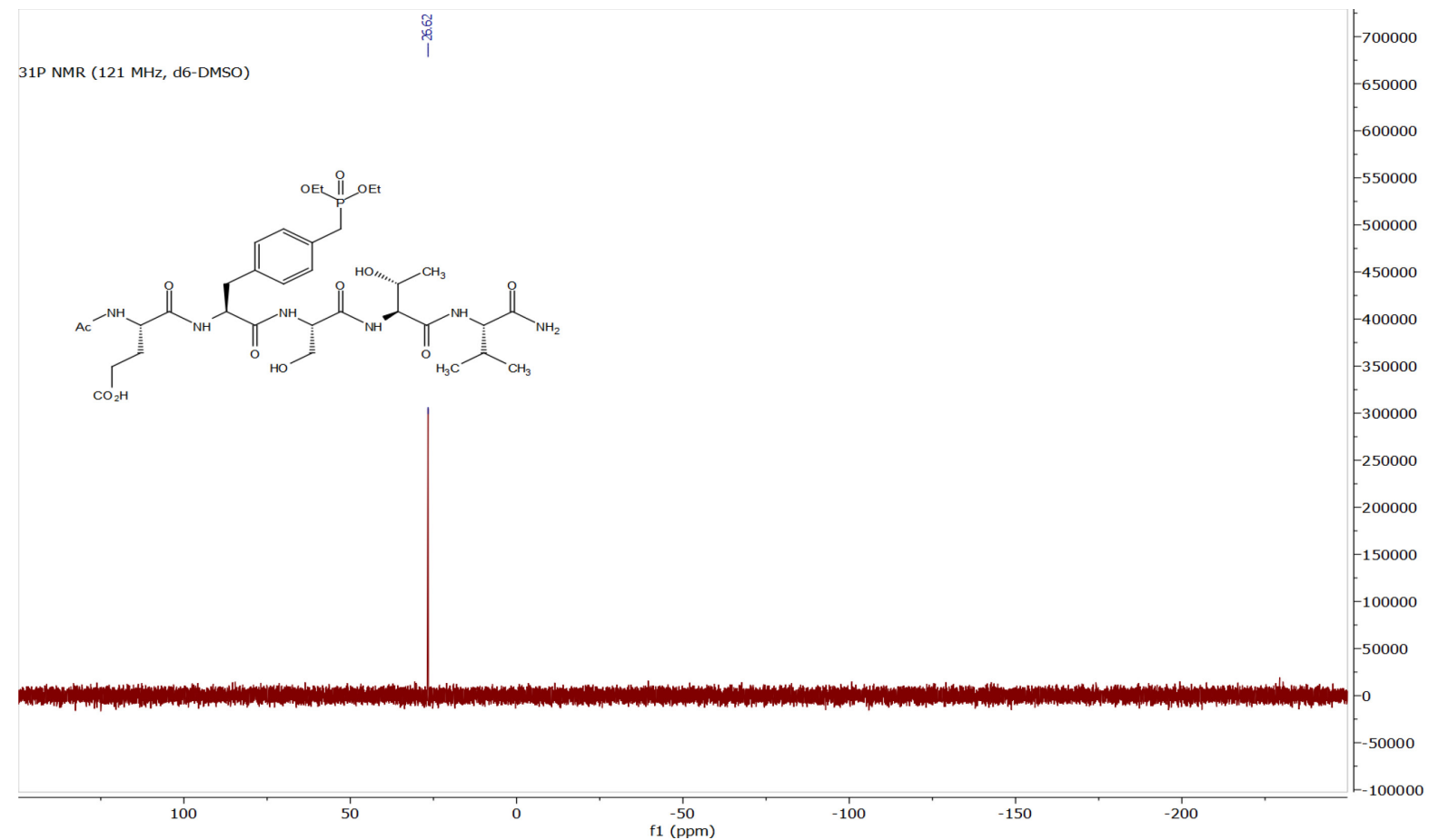

Compound was analyzed by $214 \mathrm{~nm}$ wavelength

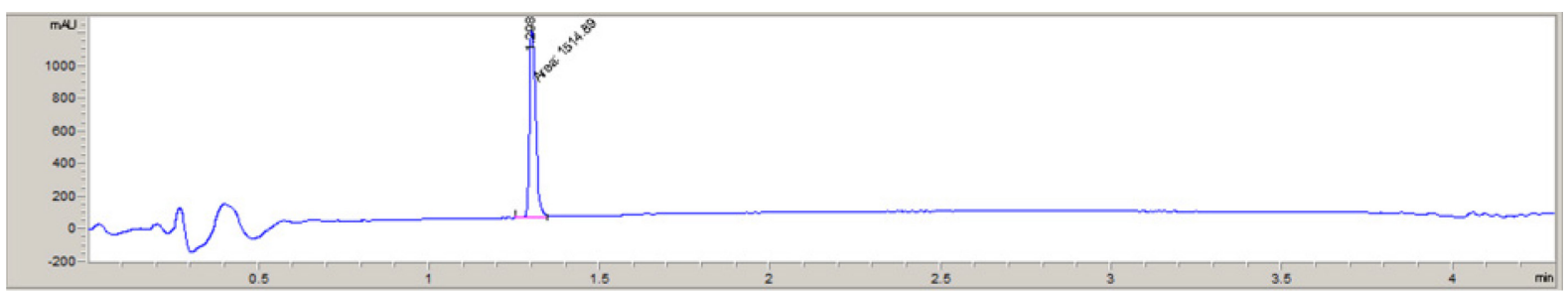


Ac-Pro-GIn-Asp-Lys-Glu-Phe(4-iodo)-Tyr-Lys-Val-Lys- $\mathrm{NH}_{2}$ (10f)

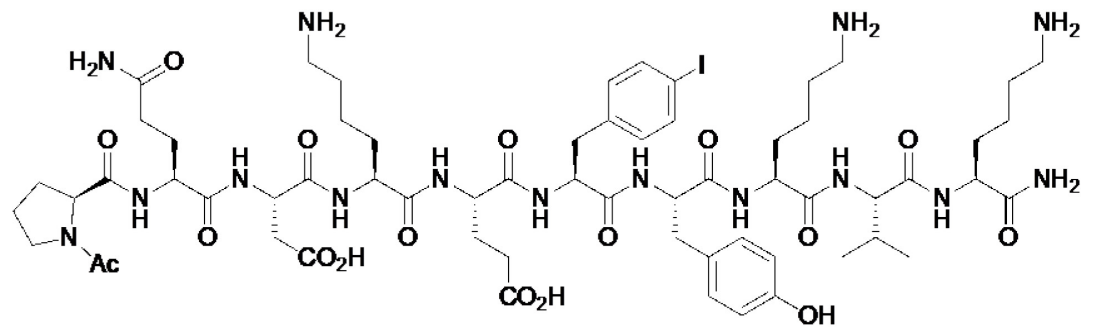

Compound was analyzed by $214 \mathrm{~nm}$ wavelength
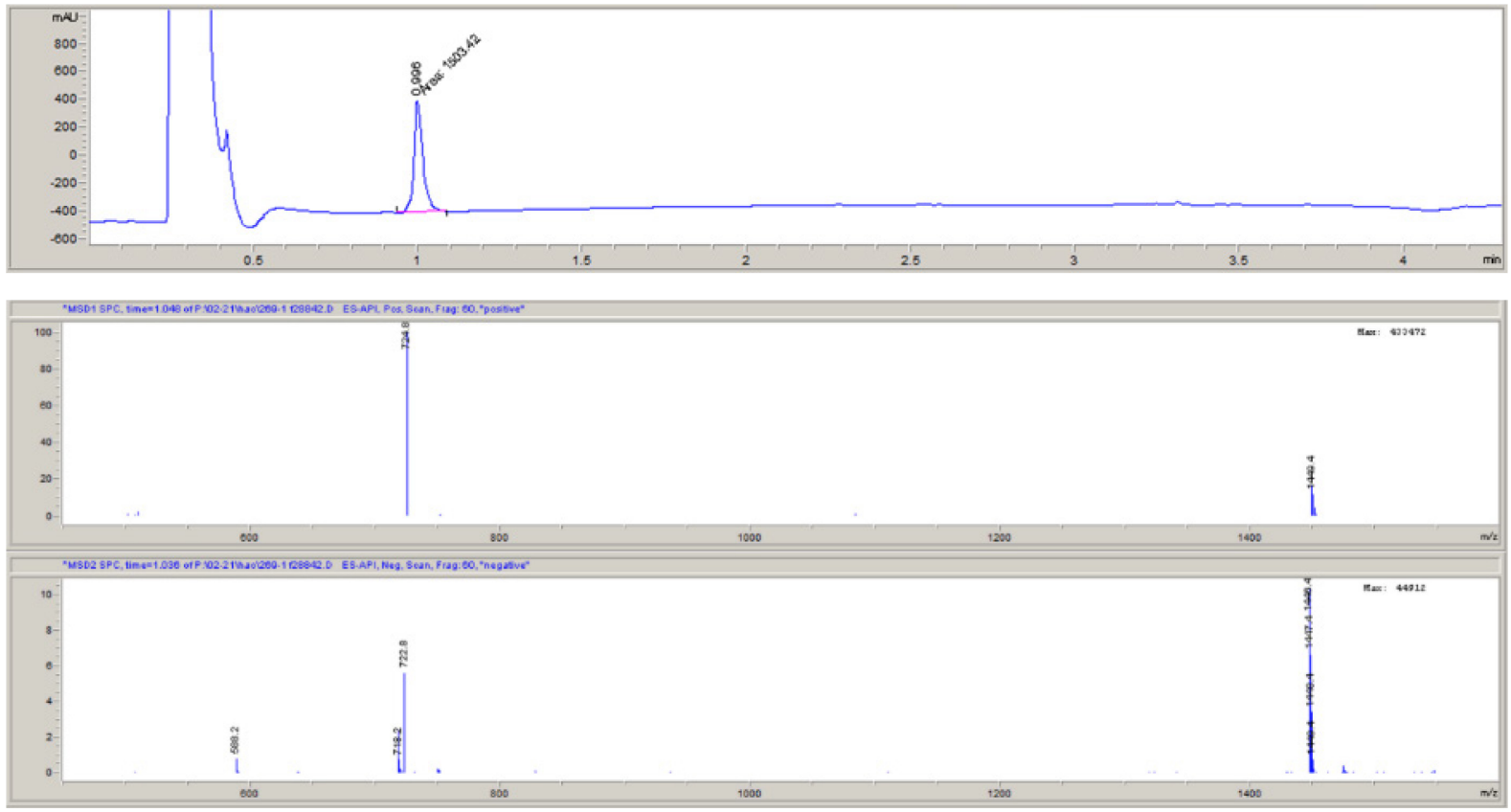
Ac-Pro-Gln-Asp-Lys-Glu- $p$ CMF(OtBu)-Tyr-Lys-Val-Lys-NH 2 (11f)

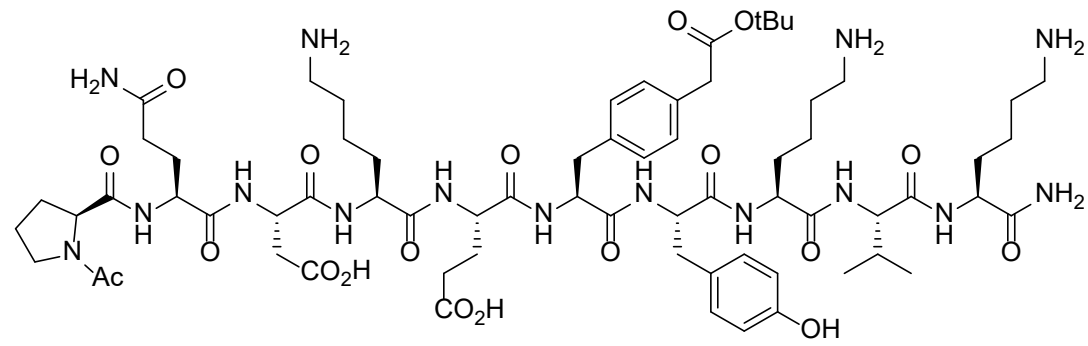

Compound was analyzed by $214 \mathrm{~nm}$ wavelength
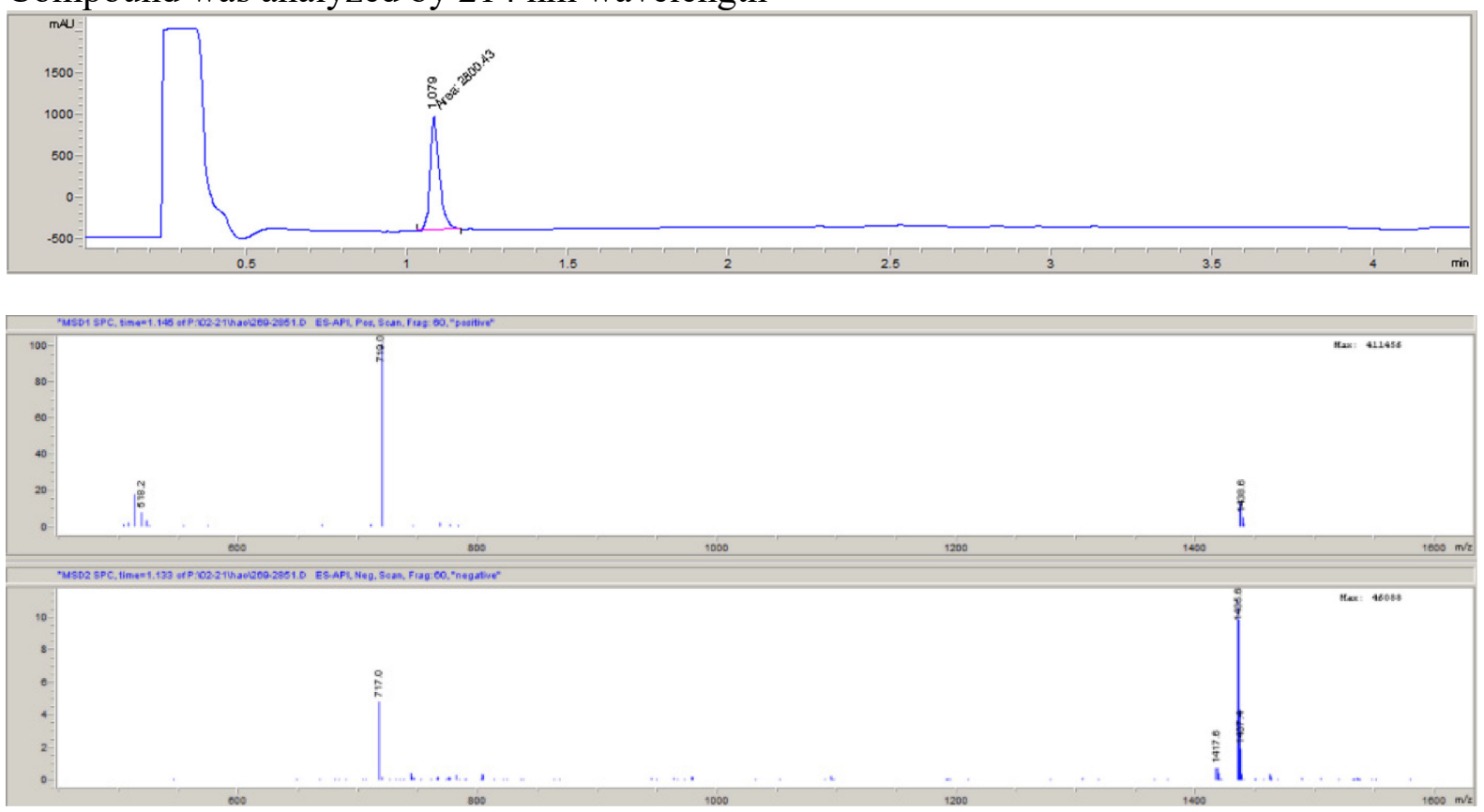


\section{Ac-Glu-Tyr $\left(\mathrm{PO}_{3} \mathrm{H}_{2}\right)$-Tyr-Lys-Val-NH 2 (12a)}

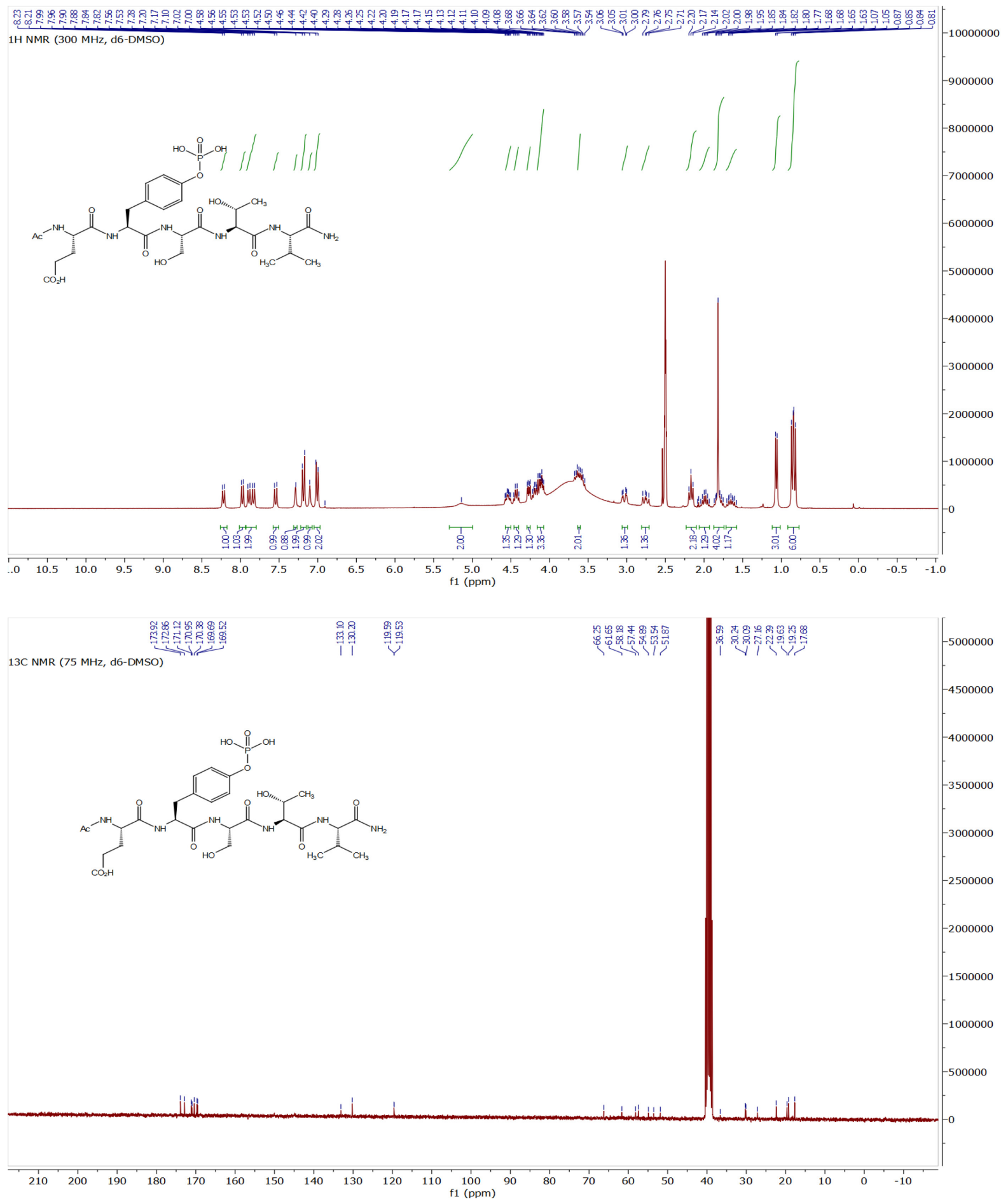




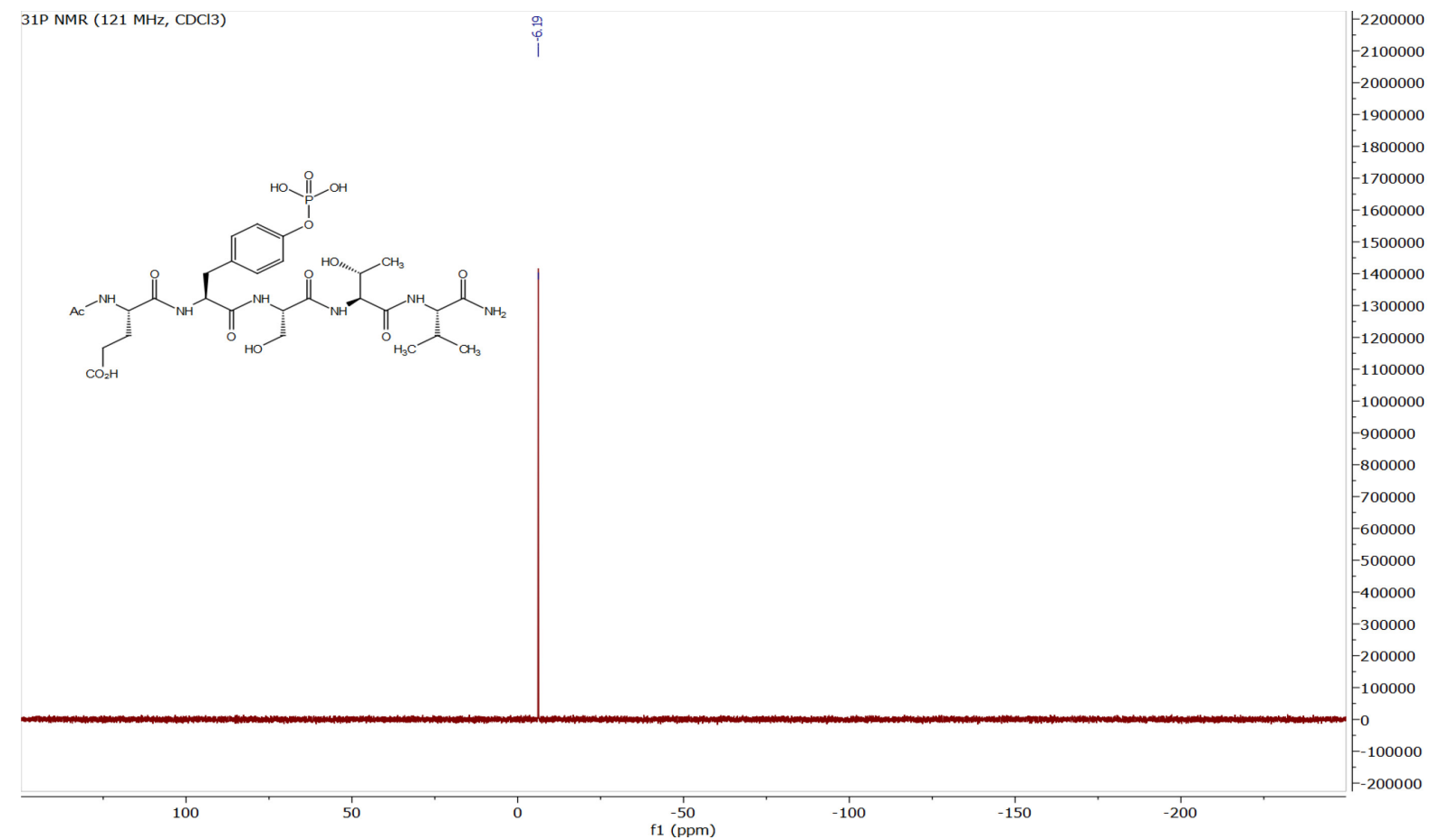

Compound was analyzed by $214 \mathrm{~nm}$ wavelength

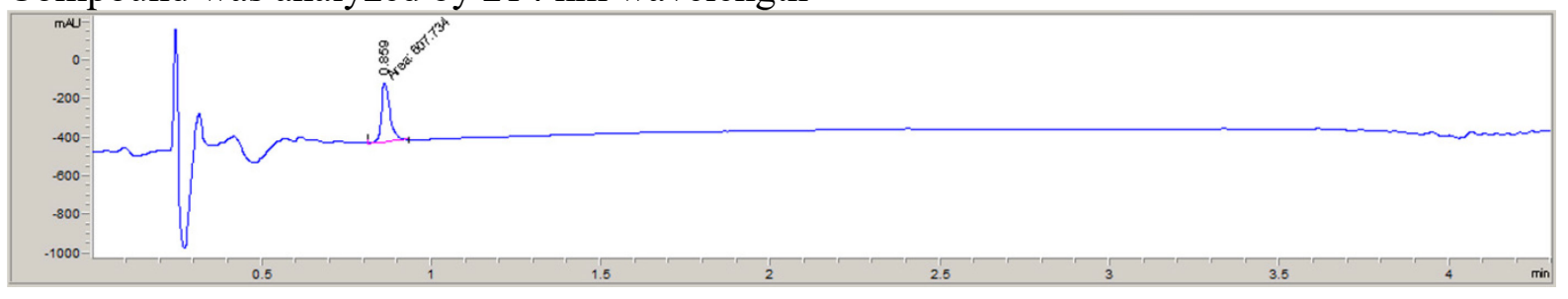




\section{Ac-Glu- $p$ CMF-Ser-Thr-Val-NH2 (12b)}

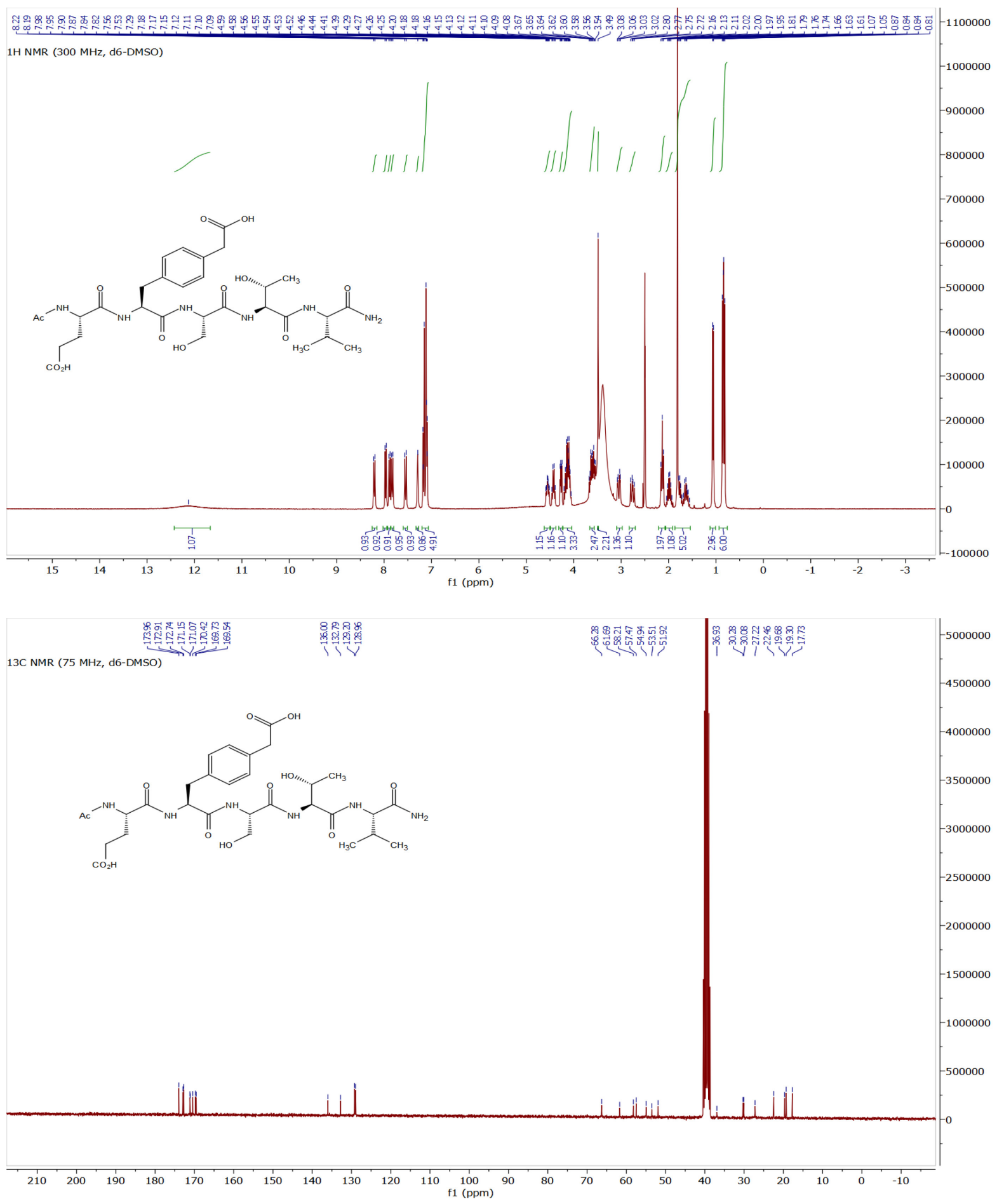




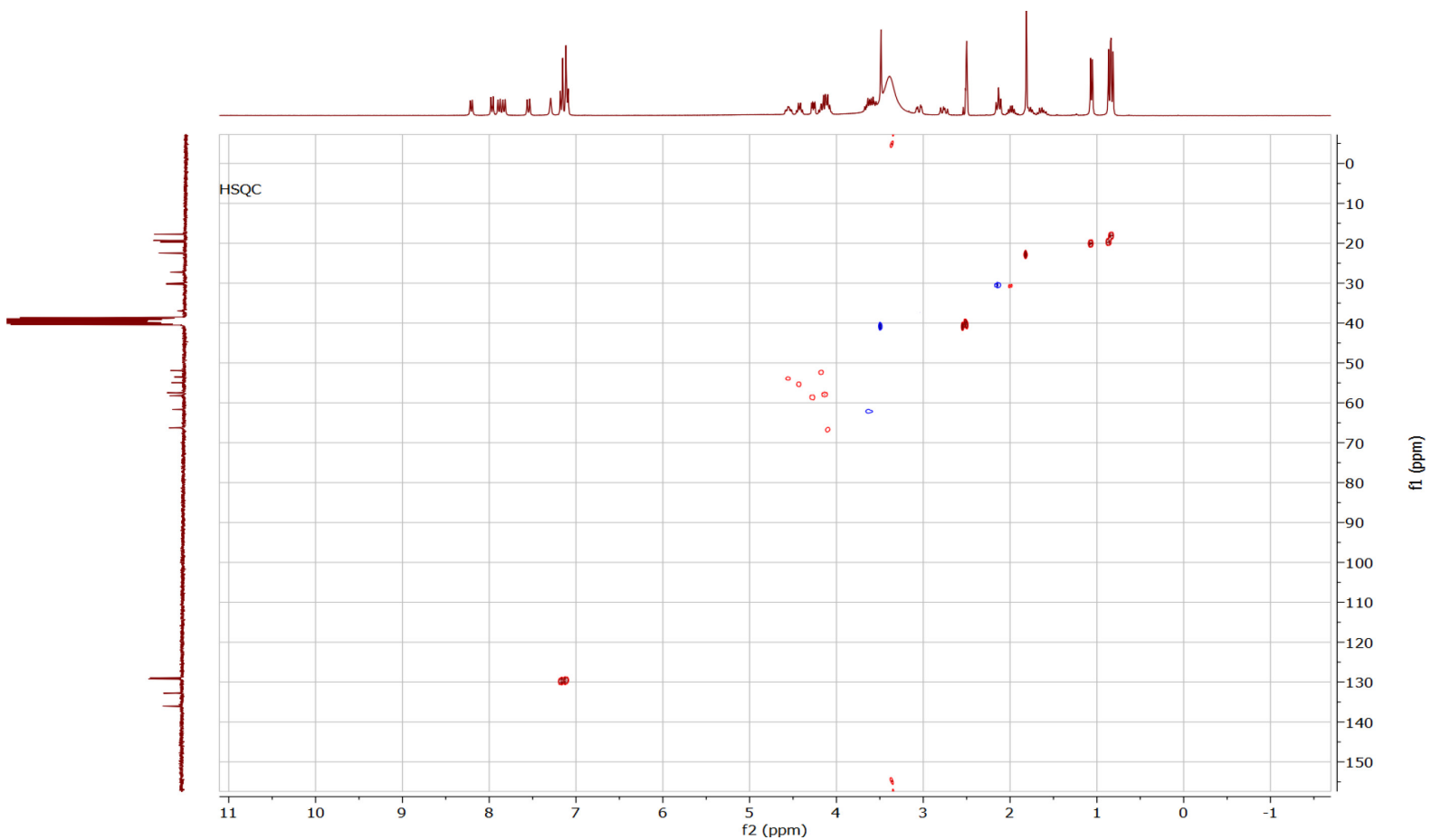

Compound was analyzed by $214 \mathrm{~nm}$ wavelength

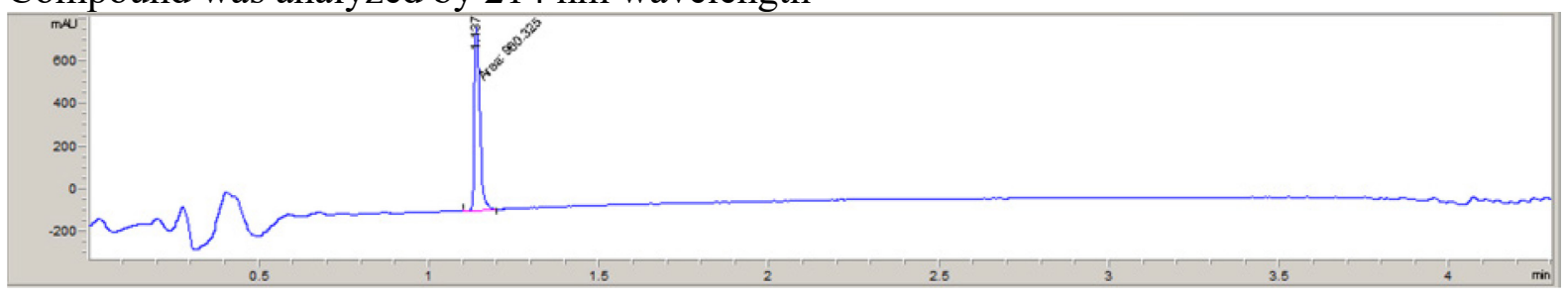




\section{Ac-Glu-Pmp-Ser-Thr-Val-NH 2 (12c)}
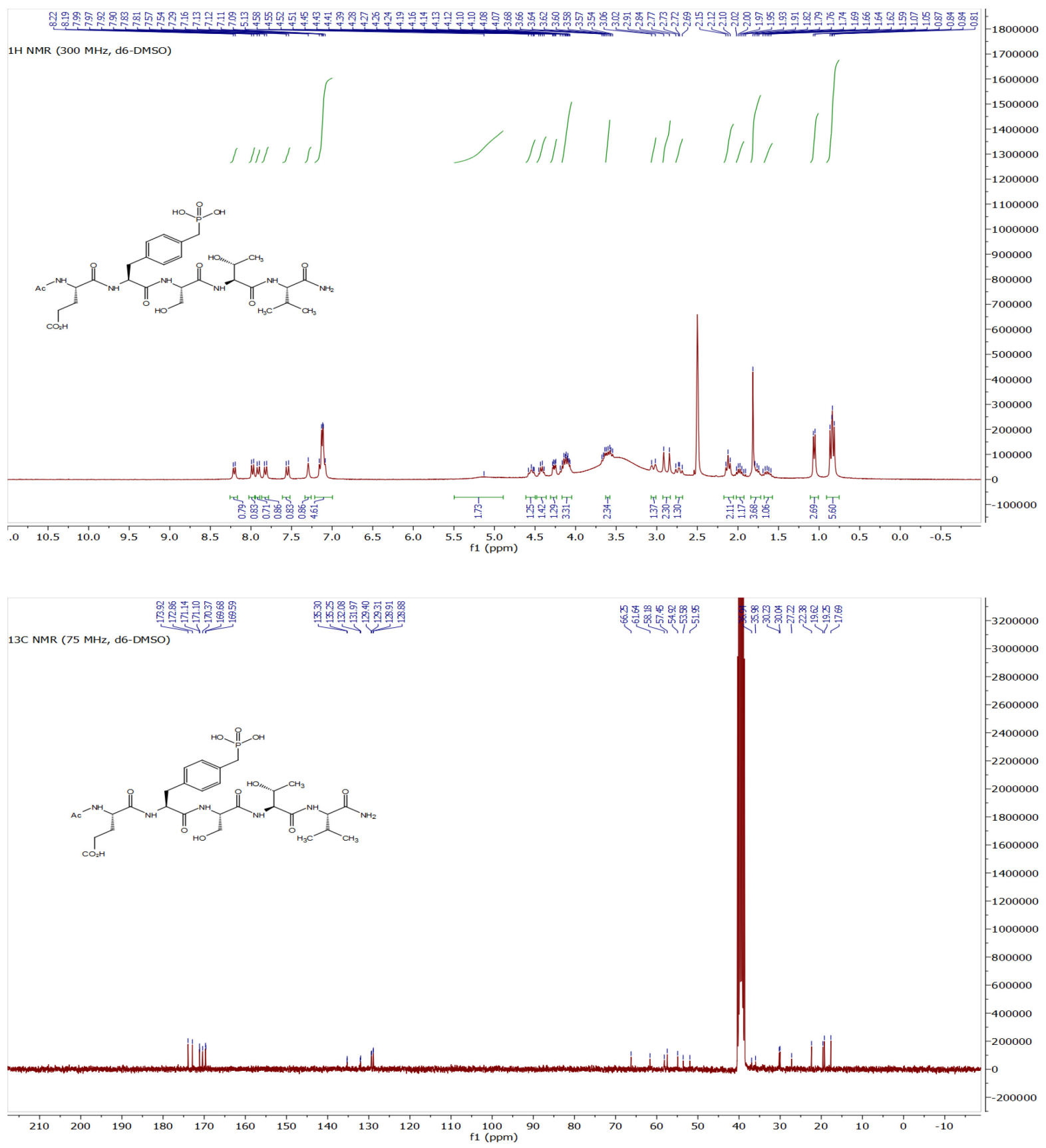


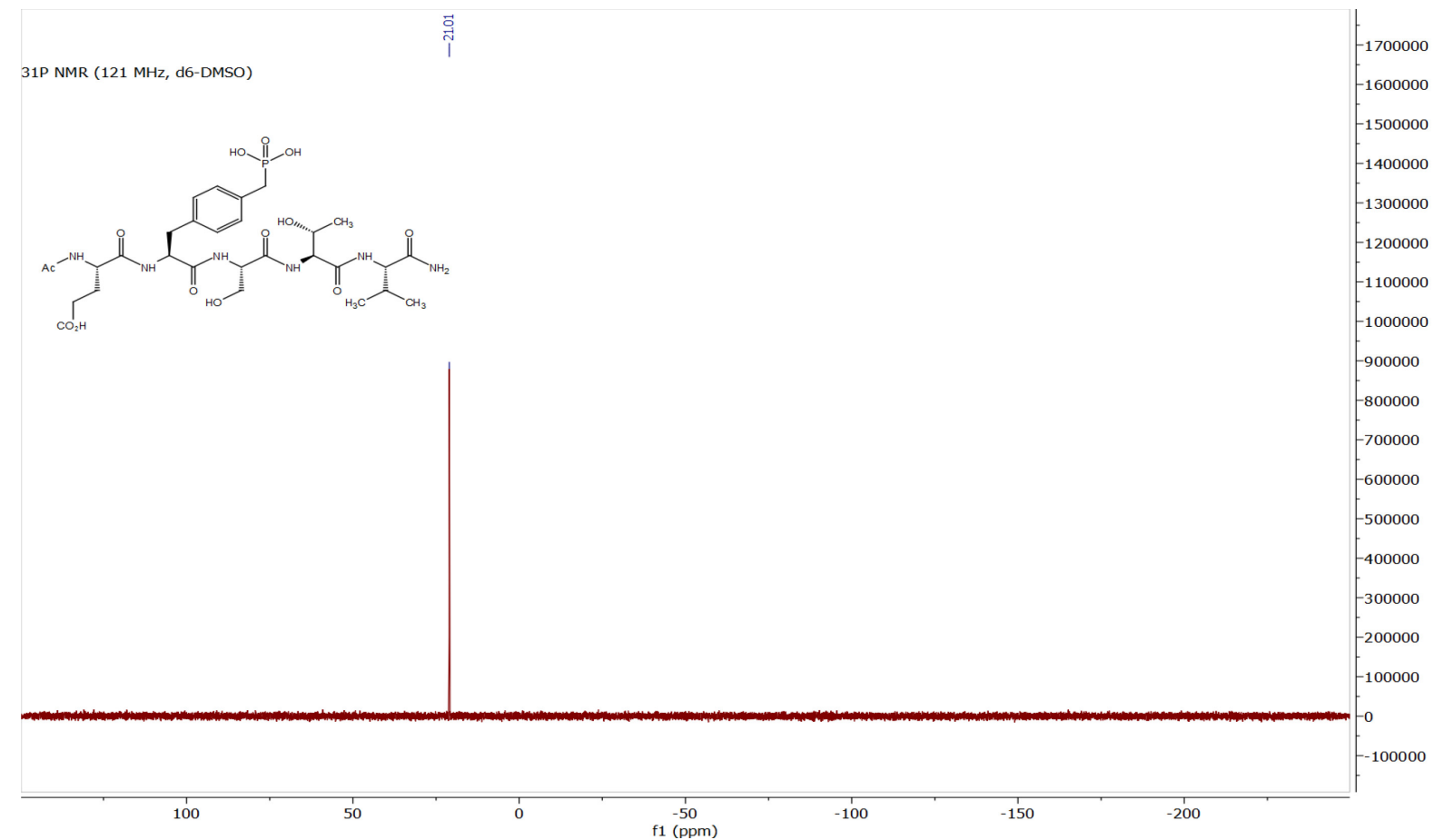

Compound was analyzed by $214 \mathrm{~nm}$ wavelength

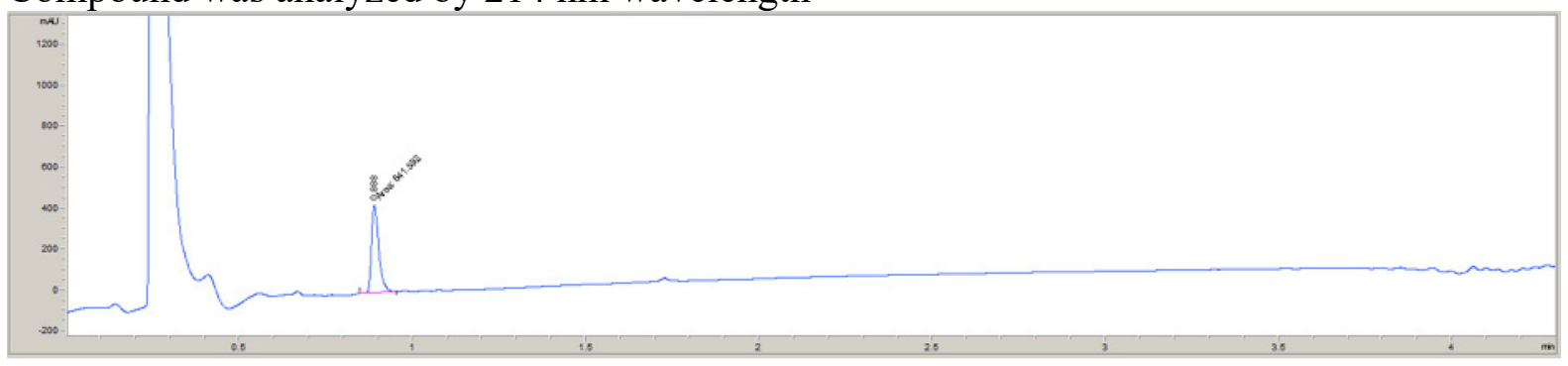


(R)-Methyl 3-(4-iodophenyl)-2-(2,2,2-trifluoroacetamido)propanoate (R1)

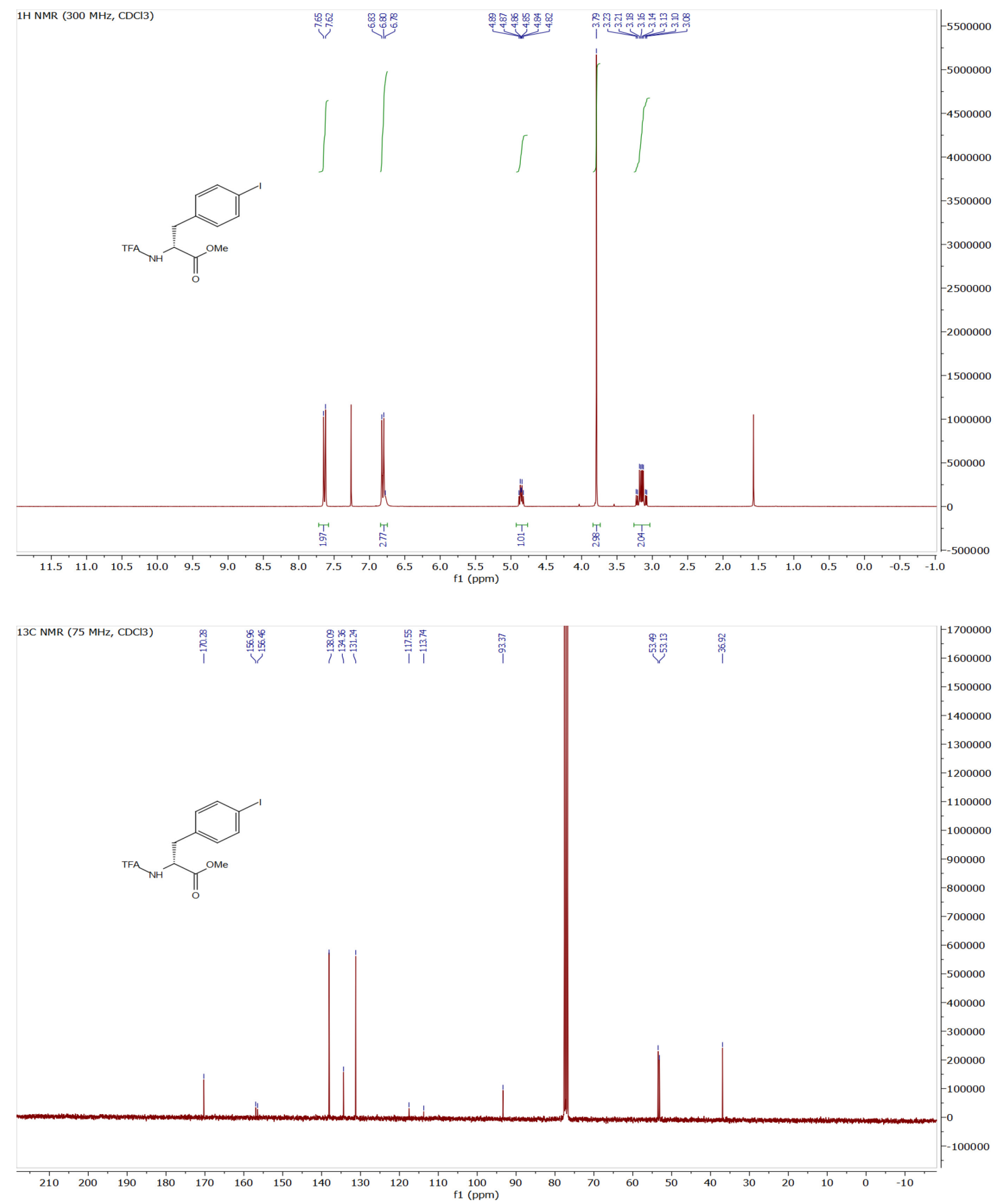




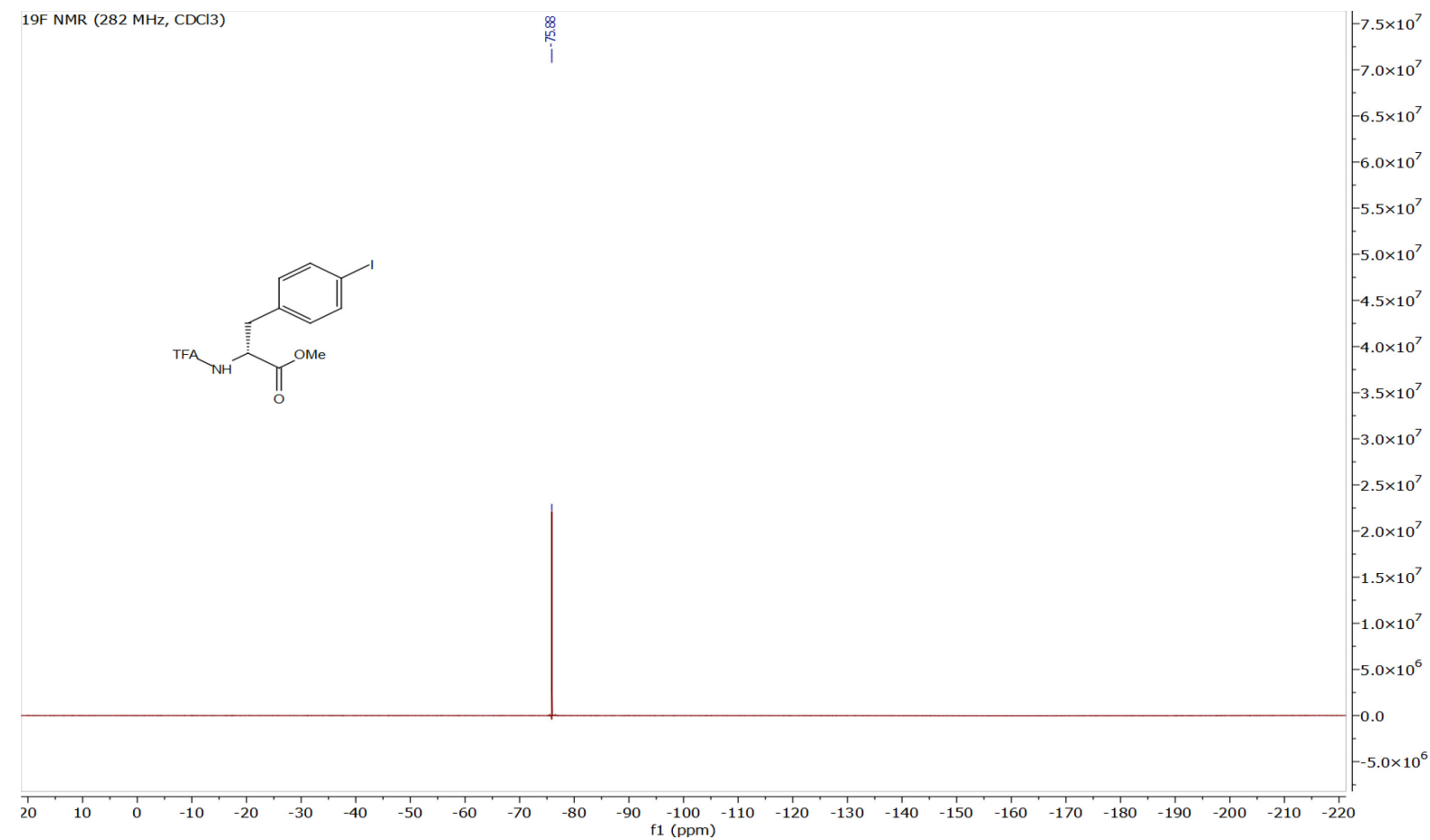

Compound was analyzed by $214 \mathrm{~nm}$ wavelength

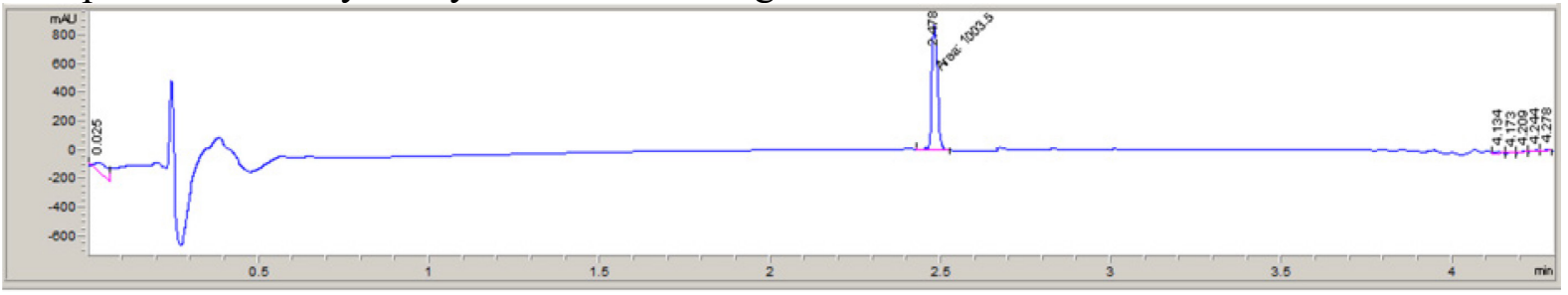


(R)-Methyl 3-(4-(2-(tert-butoxy)-2-oxoethyl)phenyl)-2-(2,2,2trifluoroacetamido)propanoate $(R 3)$

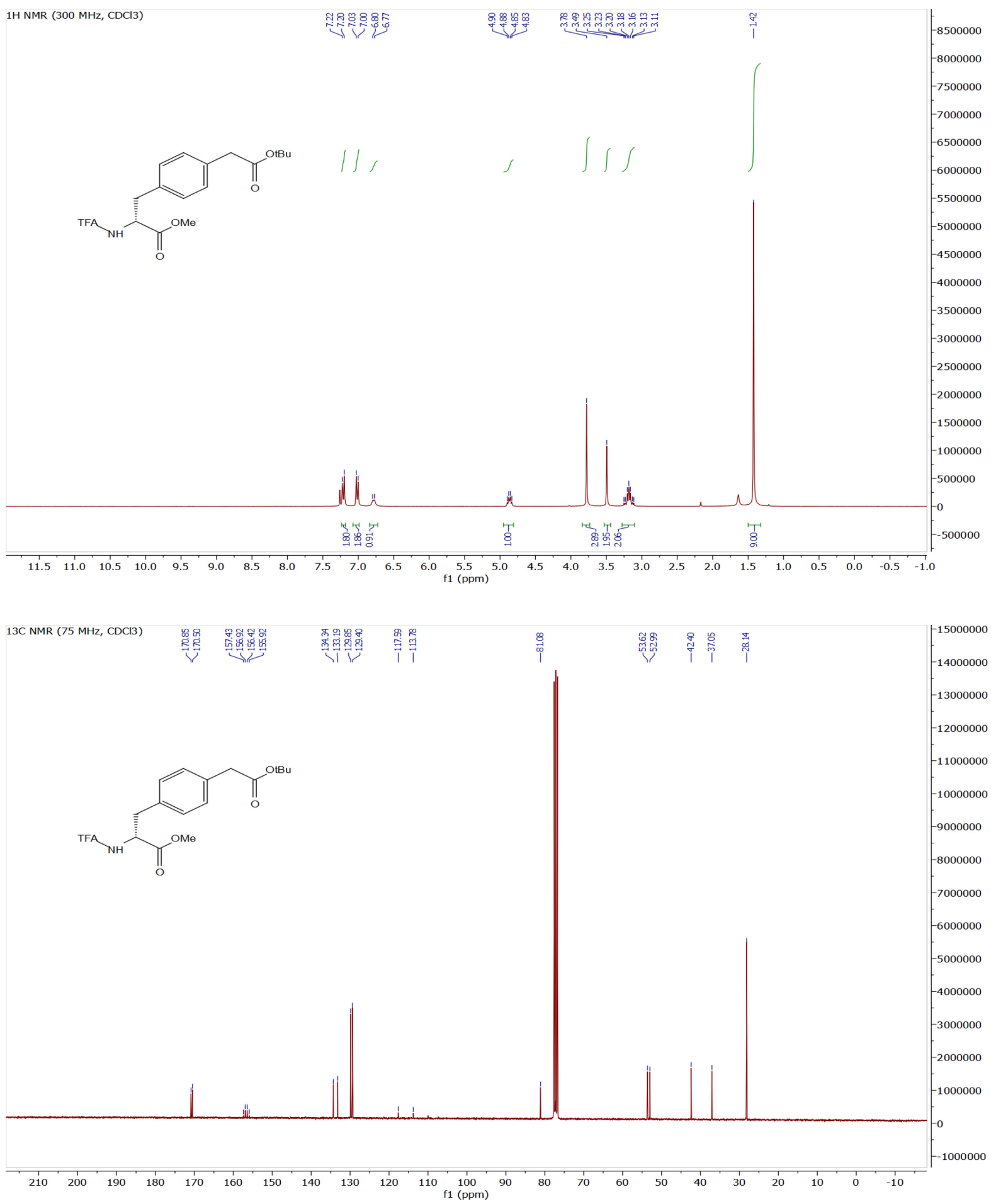




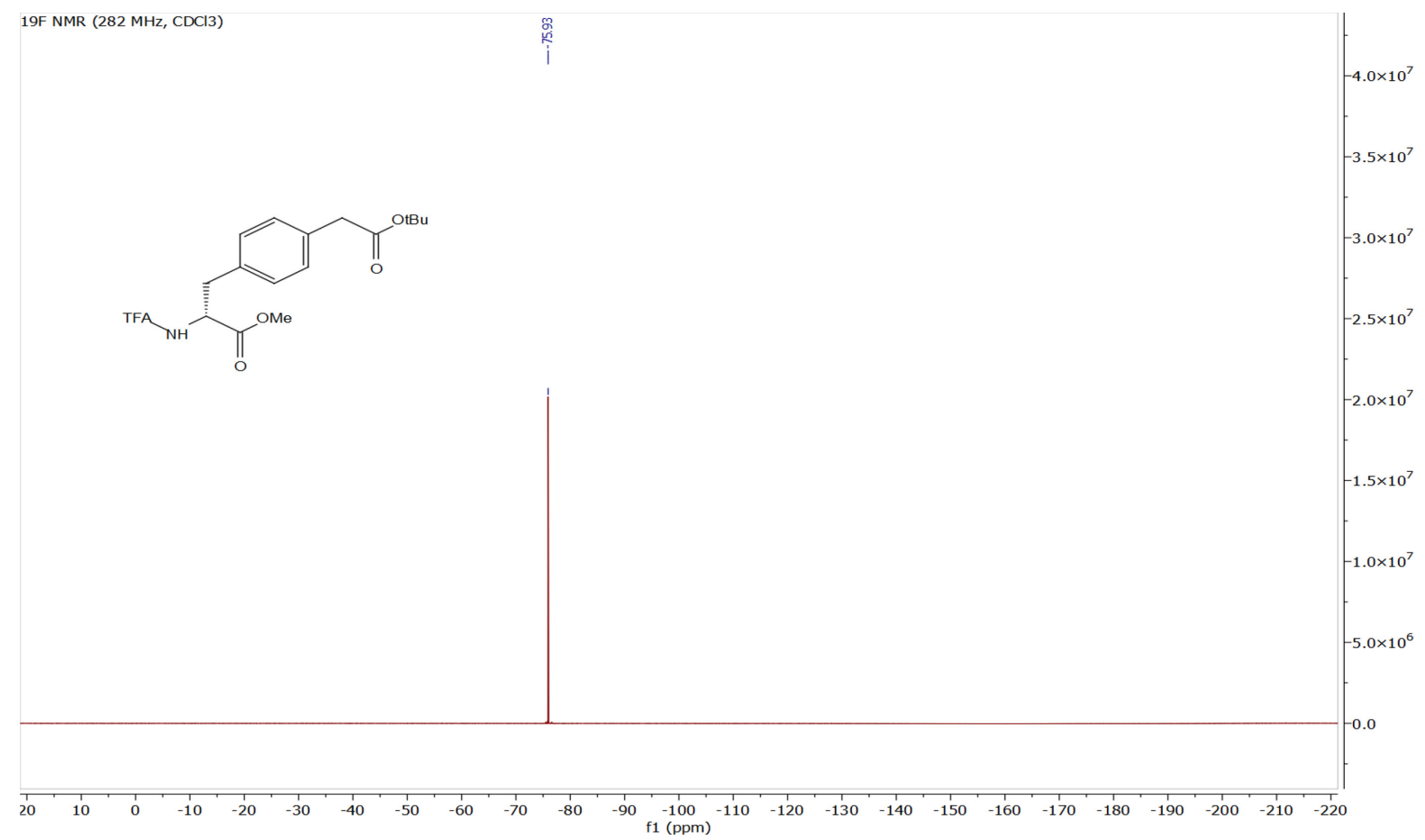

Compound was analyzed by $214 \mathrm{~nm}$ wavelength

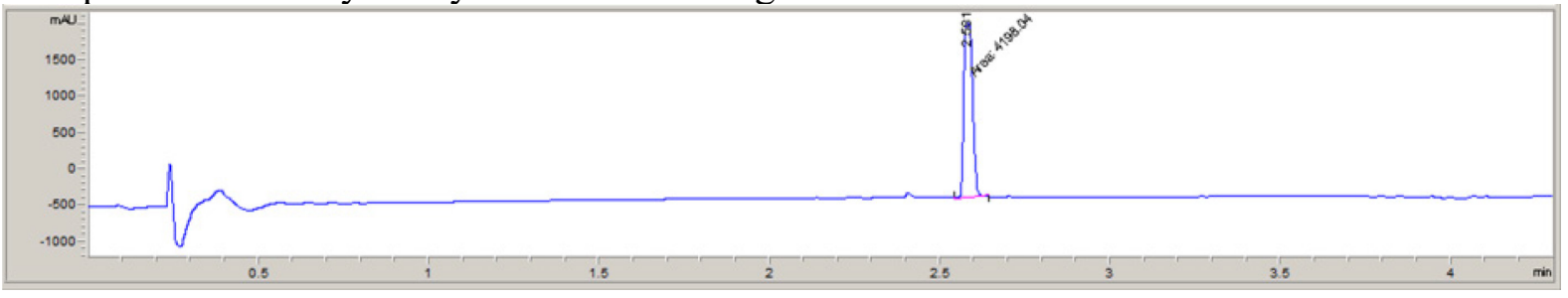


